\title{
MEASUREMENTS OF $\Gamma\left(Z^{0} \rightarrow b \bar{b}\right) / \Gamma\left(Z^{0} \rightarrow\right.$ hadrons $)$ USING THE SLD ${ }^{1}$
}

\author{
Homer Alfred Neal, Jr. II \\ Stanford Linear Accelerator Center \\ Stanford University \\ Stanford, California 94309
}

July 1995

Prepared for the Department of Energy

under contract number DE-AC03-76SF00515

Printed in the United States of America. Available from the National Technical Information Service, U.S. Department of Commerce, 5285 Port Royal Road, Springfield, Virginia 22161.

\footnotetext{
${ }^{1}$ Ph.D. Thesis
}

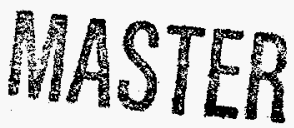




\section{Abstract}

The quantity $R_{b}=\Gamma\left(Z^{0} \rightarrow b \bar{b}\right) / \Gamma\left(Z^{0} \rightarrow\right.$ hadrons $)$ is a sensitive measure of corrections to the $Z b b$ vertex. The precision necessary to observe the top quark mass dependent corrections is close to being achieved. LEP is already observing a $1.8 \sigma$ deviation from the Standard Model prediction. Knowledge of the top quark mass combined with the observation of deviations from the Standard Model prediction would indicate new physics. Models which include charged Higgs or light SUSY particles yield predictions for $R_{b}$ appreciably different from the Standard Model. In this thesis two independent methods are used to measure $R_{b}$. One uses a general event tag which determines $R_{b}$ from the rate at which events are tagged as $Z^{0} \rightarrow b \bar{b}$ in data and the estimated rates at which various flavors of events are tagged from the Monte Carlo. The second method reduces the reliance on the Monte Carlo by separately tagging each hemisphere as containing a $b$-decay. The rates of single hemisphere tagged events and both hemisphere tagged events are used to determine the tagging efficiency for $b$-quarks directly from the data thus eliminating the main sources of systematic error present in the event tag. Both measurements take advantage of the unique environment provided by the SLAC Linear Collider(SLC) and the SLAC Large Detector(SLD). From the event tag a result of $R_{b}=0.230 \pm 0.004_{\text {statistical }} \pm 0.013_{\text {systematic }}$ is obtained. The higher precision hemisphere tag result obtained is $R_{b}=0.218 \pm 0.004_{\text {statistical }} \pm 0.004_{\text {systematic }} \pm 0.003_{R_{c}}$. 


\section{DISCLAIMER}

Portions of this document may be illegible in electronic image products. Images are produced from the best available original document. 


\section{Acknowledgments}

At SLAC I have seen the SLD experiment evolve from its pre-physics producing stage to a significant contributer of results that interest the scientific community. It has been an experience beyond what I had hoped and one that will be sincerely missed. The people that I've had the fortune of working with have made the experience particularly special. These people have provided friendship, knowledge, and guidance that will not be forgotten.

Foremost, I owe much gratitude to my advisor, Rafe Schindler, for his guidance and encouragement. I have benefited much from his wisdom and his example. Ultimately, I owe my whole SLD experience to him.

Special thanks goes to Su Dong with whom I have worked with closely over the past couple of years. Through him I gained much of my knowledge of $b$-physics and have gained a better appreciation of critical analysis. I have always considered him as a friend and teacher.

Tom Junk has been an ideal office-mate for the many years I've spent at SLAC as a student. I have greatly appreciated his kindness and wit. It is through discussions with him that many of my concepts on the experiment and on physics in general were clarified.

Thanks to Marty Breidenbach for always finding time to share his experiences with me and to provide guidance.

Great appreciation goes to Sarah Hedges for being the best e-mail and tennis pal a guy could ask for. Her support and friendship are largely responsible for the enjoyment of my years at SLAC. Special thanks also goes to Jenny Huber for her friendship and good humor. I have enjoyed the many conversations with Mike Hildreth, Tom Pavel, Ken Baird, Peter Tannenbaum, John Labs, Hiro Masuda, Jingchen Zhou, Pablo Saez, Nancy Mar and Hwanbae Park.

One of the most enjoyable tasks on SLD has been the effort to improve the tracking simulation and reconstruction. The people I've work with in this effort have become good friends. Especially, I would like to thank Tracy Usher who has taught me much about tracking and the operation of the drift chamber. In addition, he has also been 
a major motivator in improving my skiing skills. I hope to see him on the slopes during the endless winters ahead. Thanks to Leon Rochester, Tom Markiewicz, and David Williams for their friendship and knowledge of tracking.

Thanks to the very deserving crew of people who have successfully kept the computers running despite my occasional liberal use any available cpu. Among the crew are Richard Dubois, Tony Johnson, Ramon Berger, Karen Heidenrech, Dave Sherden, Owen Saxton, Joe Perl and Gary Bower. Immense thanks goes to those who have designed and maintained the SLD acquisitions systems. This includes Jim Russell, Mike Huffer, and Jack Hoeflich.

Thanks to Margret Helton for always providing excellent advise on the more important aspects of life. Thanks to Barbara Barrera, Mary Brau and Lydia Beers for being extremely helpful in preparing meetings, conferences and publications.

Several people were particularly helpful in starting my career at SLAC. I would like to thank Dave Burke who was my advisor during my research rotation at SLAC and helped to fortify my interest in particle physics. Thanks to Walter Meyerhof for welcoming me to Stanford and for his thoughtful consideration and support.

Thanks to Iris Abt and Eric Vella for their friendship and knowledge of calorimetry. During the early years of SLD when data was more than a little scarce and a SLD thesis was seeming more and more unlikely, they provided me with hardware and software experience that has continued to be useful throughout the more prolific years of SLD. Thanks to Tony Waite for giving me a better appreciation of structured programming.

The staff of SLD and group E has been a pleasure to work with and I'm especially thankful for the respect they have shown me. These include Bruce Schumm, Mary King, Bill Wisniewski, Charlie Baltay, Terry Schalk, Bob Messner, Chris Damerell, Jim Brau, John Jaros, Steve Manly, Dave Muller, Phil Burrows, Peter Rowson and Steve Wagner.

Over the last couple of years it has been a sincere pleasure to work and learn with many new students that have joined the experiment. In particular I'd like to thank Xiaoqing Yang, Eric Weiss, John Coller, Hyejoo Kang, Giampiero Mancinelli, Donatella Falciai, Eric Church and Aaron Chou. Apologies, to anyone I may have 
forgotten.

All members of the SLD Heavy Flavor Physics Group have been significant contributers to my development as a physicist. Many thanks for their support. It has also been a pleasure getting to know all the member of Group $\mathrm{E}$.

My first experience at SLAC actually occurred nearly 15 years ago when my father was working on the HRS experiment. Before I had had my first high school physics course I had already visited many of the major high energy physics research facilities due to his interest. It is from this exposure to the field that $I$ have grown to love it and decided to choose it as my profession. Much gratitude goes to my father for the many great experiences he has shared with me.

My parents, Mr. and Mrs. Homer A. Neal, my sister, Sharon Brangman, my aunt, Eddaline Bannister, and uncle, Donald Daniels are especially deserving of thanks for all of the support they have provided during my entire life. Also, special thanks goes to my brother-in-law, Stephen Brangman for his support. It is to them that I dedicate this thesis.

I know I will have missed some very deserving people in these acknowledgments. Please know that it is unintentional and you will always be greatly appreciated for your part in my life. Thanks to all! 


\section{Contents}

1 Introduction 1

1.1 Motivation for Performing Measurement using the SLD . . . . . . 3

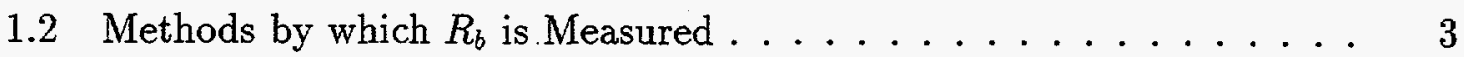

$1.3 \quad b$-Tags used in this Thesis $\ldots \ldots \ldots \ldots \ldots \ldots$

2 Theory 6

2.1 The Standard Model . . . . . . . . . . . . . . . 6

2.1.1 Fundamental Particles . . . . . . . . . . . 7

2.1 .2 Gauge Bosons . . . . . . . . . . . . . . . . 8

2.1.3 Strong Interactions and Hadrons $\ldots \ldots \ldots \ldots$

2.1.4 $B$-Hadrons and Their Decays $\ldots \ldots \ldots \ldots \ldots$

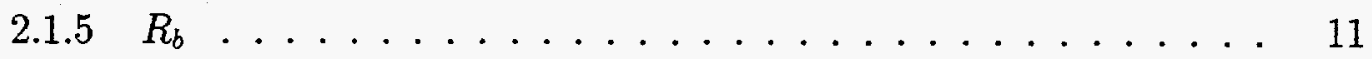

2.1.6 Top Quark Mass Dependence . . . . . . . . . . 13

2.1.7 Supersymmetric Extensions . . . . . . . . . . . 16

2.2 Theoretical Uncertainties in $R_{b} \ldots \ldots \ldots \ldots \ldots \ldots$

2.2 .1 Gluon Splitting . . . . . . . . . . . . . . 19

3 Exp. Apparatus \& Detector Simulation 21

3.1 The SLAC Linear Collider . . . . . . . . . . . . . . . . 22

3.1 .1 Beam Energy . . . . . . . . . . . . . . . . 24

3.2 The SLAC Large Detector (SLD) $\ldots \ldots \ldots \ldots \ldots \ldots$

3.2.1 The SLD Coordinate System . . . . . . . . . . . . . 26

3.2 .2 The Central Drift Chamber . . . . . . . . . . 27 
3.2.3 The SLD Vertex Detector . . . . . . . . . . . . . . 34

3.2.4 The Liquid Argon Calorimeter $\ldots \ldots \ldots \ldots . \ldots 38$

3.2 .5 Data Acquisition . . . . . . . . . . . . 40

3.2 .6 SLD Runs and Run Number . . . . . . . . . . . . . 40

3.3 Detector Simulations . . . . . . . . . . . . . . . . . 42

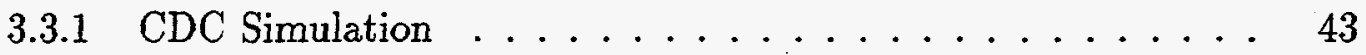

3.3 .2 VXD Simulation $\ldots \ldots \ldots \ldots \ldots \ldots \ldots$

3.3 .3 LAC Simulation . . . . . . . . . . . . . . . 46

3.4 Detector Performance Checks . . . . . . . . . . . . 46

3.4.1 Checks using $Z^{0} \rightarrow \mu^{+} \mu^{-}$Events $\ldots \ldots \ldots \ldots \ldots$

3.4.2 Tracking Performance vs. Monte Carlo Prediction . . . . . . 48

4 Primary Vertex Position Measurement 54

4.0.3 Determining Transverse Components of the Primary Vertex . 56

4.0.4 Determining the Longitudinal Component of the Primary Vertex 57

5 Tagging Variables $\quad 59$

5.1 The 2-D Impact Parameter . . . . . . . . . . . . . . . . . 59

5.1 .1 Impact Parameter Signing . . . . . . . . . . . 60

5.1.2 Normalizing the Impact Parameter . . . . . . . . . . . 61

5.1.3 Contributions to the Impact Parameter Uncertainty . . . . . 62

5.2 The Minimized 3-D Impact $\operatorname{Parameter}(\chi) \ldots \ldots \ldots 66$

6 Physics Simulation $\quad 72$

6.1 Simulation of $B$-Hadron Decays . . . . . . . . . . . . 72

6.2 Simulation of Charm Hadron Decays . . . . . . . . . . . 73

6.3 QCD Simulation Parameters . . . . . . . . . . . . . . 74

7 Event and Track Selection $\quad 79$

7.1 Hadronic Event Selection . . . . . . . . . . . . . . . . . 79

7.1 .1 Event Triggers . . . . . . . . . . . . . 79

7.1 .2 Event Selection . . . . . . . . . . . . . . 80 
7.2 Track Selection . . . . . . . . . . . . . . . . 83

7.3 Tracking Efficiency Corrections _ . . . . . . . . . . 85

7.4 Tracking Resolution Corrections . . . . . . . . . . . . . 88

8 The 2-D Impact Parameter Event Tag 92

8.1 Introduction . . . . . . . . . . . . . . . . 92

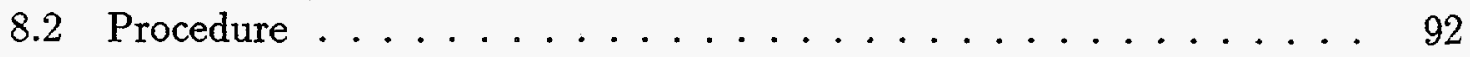

8.2.1 Determining $R_{b}$ from Event Tag Counts $\ldots \ldots \ldots \ldots 94$

8.2 .2 Statistical Uncertainties $\ldots \ldots \ldots \ldots \ldots$. . . . . . 95

8.2 .3 The Tag . . . . . . . . . . . . . . . 97

8.3 Measurement Results . . . . . . . . . . . . . . . . 97

8.4 Systematics . . . . . . . . . . . . . . . . . . . 99

8.4.1 Detector Systematics . . . . . . . . . . . . . 100

8.4 .2 Physics Systematics . . . . . . . . . . . . . . . . . . 101

8.5 Verifications . . . . . . . . . . . . . . 107

8.6 Final Result . . . . . . . . . . . . . . . . 108

9 The Lifetime Probability Tag 109

9.1 Introduction . . . . . . . . . . . . . . . . . . . 109

9.1.1 Outline of the Probability Tagging Procedure . . . . . . 110

9.2 Event and Track Selection Modifications . . . . . . . . . . . . 112

9.2.1 Event Selection and Counts . . . . . . . . . . . 112

9.2 .2 Track Selection . . . . . . . . . . . . . . . . 114

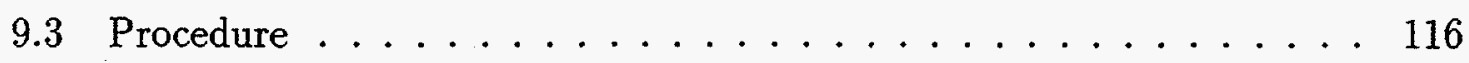

9.3.1 Resolution Corrections . . . . . . . . . . . . . . . 119

9.3.2 Determining $R_{b}$ from Tag Counts . . . . . . . . . . 119

9.3.3 Statistical Uncertainties with Correlation Effects Included . . 124

9.4 Measurement Results . . . . . . . . . . . . . . . . . 125

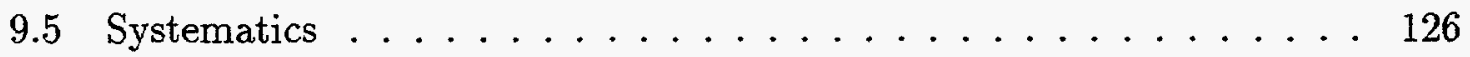

9.5 .1 Physics Systematics . . . . . . . . . . . . . . 126

9.5 .2 Detector Systematics . . . . . . . . . . . . . . . . 129

9.5 .3 Verifications . . . . . . . . . . . . . . 132 
9.5.4 Check of correlation components from data . . . . . . 140

9.5.5 Summary of Systematics . . . . . . . . . . . . . . 142

9.6 Final Result . . . . . . . . . . . . . . . . . . . . . . 142

10 Implications and Future Prospects 144

A $V^{0}$ Rejection and Material Simulation Checks $\quad 147$

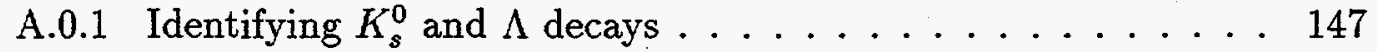

A.0.2 Identifying Gamma Conversions . . . . . . . . . . . . . 149

A.0.3 Using Conversions to test Monte Carlo Material Simulation . . 150

B Other Procedures for Measuring $R_{b}$ at SLD 154

B.0.4 3-D Impact Parameter Event Tag . . . . . . . . . . . . . 154

B.0.5 3-D Displaced Vertex Tag $\ldots \ldots \ldots \ldots \ldots \ldots$

B.0.6 Impact Parameter Sum . . . . . . . . . . . . . . 156

C Drift Chamber Monitoring and Calibrations 158

C. 0.7 Drift Velocity Constants . . . . . . . . . . . 158

C.0.8 Charge Asymmetry Calibrations . . . . . . . . . . . 163

D Effect of Backgrounds on Tracking 164

E The SLD Collaboration $\quad 166$ 


\section{List of Tables}

2.1 Fundamental particle doublets. . . . . . . . . . 7

2.2 Fundamental particle singlets. . . . . . . . . . . 7

2.3 Vector and axial coupling strengths for the neutral current interaction. 9

3.1 SLC operating performance. . . . . . . . . . . . . . . 24

3.2 Run numbers corresponding to the three SLD runs. . . . . . . . 42

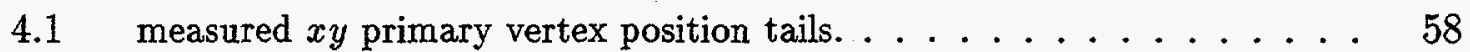

5.1 SLD and ALEPH intrinsic impact resolutions. [50] . . . . . . . 63

5.2 Radiation lengths seen by a track passing through the central tracking volume at $\theta=90^{\circ} \ldots \ldots \ldots \ldots \ldots \ldots \ldots \ldots \ldots$

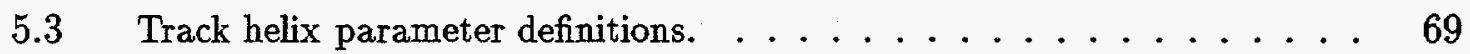

6.1 Distribution of the $B$ decay branching fraction. . . . . . . . 73

6.2 Summary of the charm decay model. . . . . . . . . . 77

6.3 Charmed Meson Lifetimes from 1995 PDG . . . . . . . . . . . 77

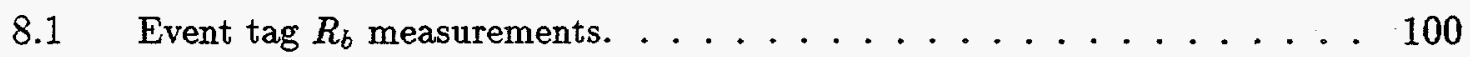

8.2 Performance of the 2-D Impact Parameter Event Tag with a cut of 3 tracks with significance $>3.0$. The uncertainties are statistical only. . . 100

8.3 Summary of detector systematics for the 2-D Impact Parameter Event Tag with a cut of 3 tracks with significance $>3.0 \ldots \ldots \ldots 2 \ldots$

8.4 Summary of b-physics systematics for the 2-D Impact Parameter Event Tag with a cut of 3 tracks with significance $>$ 3.0 . . . . . . 103

8.5 Variations applied to charm hadron production. . . . . . . . . . 104

8.6 Variations applied to charm hadron decay multiplicities. . . . . . . 105 
8.7 Summary of physics systematics, other than those directly resulting from the $b$-physics simulation, for the 2-D Impact Parameter Event Tag with a cut of 3 tracks with significance $>3.0 \ldots \ldots \ldots \ldots \ldots$

8.8 Measurements of $R_{b}$ from the 2-D Impact Parameter Event Tag versus orientation of the thrust axis. The errors are statistical only. . . . 108

9.1 Parameters of resolution function fit. . . . . . . . . . 116

9.2 Event selection efficiencies. . . . . . . . . . . . . . . . . . 124

9.3 Probability tag performance for $P_{c u t}=10^{-2.8} \ldots \ldots \ldots \ldots \ldots 142$

9.4 Summary of physics systematics for probability tag at a cut of $P_{c u t}=10^{-2.8} 143$

9.5 Summary of detector systematics for probability tag at a cut of $P_{c u t}=10^{-2.8} 143$ 


\section{List of Figures}

1.1 LEP $R_{b}$ Measurements from Moriond '95 Electroweak Conference. Uncertainty from $R_{c}$ not included. . . . . . . . . . . . . 2

1.2 An SLD $Z^{0}$ event showing reconstructed charged tracks forming two distinct secondary vertices. This is likely a $Z^{0} \rightarrow b \bar{b}$ event. . . . . . . . 4

2.1 Vertex corrections to $\Gamma_{Z b b}$ from $t$ and $W \ldots \ldots \ldots 11$

2.2 An oblique correction to $\sigma\left(Z^{0} \rightarrow b \bar{b}\right) \ldots \ldots \ldots \ldots$

2.3 Top mass dependence of $R_{b}$ for the Standard Model(MSM), the Minimal Supersymmetric Extension of the Standard Model (MSSM), and the Standard Model with a second Higgs doublet. Corrections that have not been applied would lower the curves. The deviations between models remain unchanged when all corrections are applied.[4] . . . . . . . . . 15

2.4 Vertex corrections to $\Gamma_{Z b b}$ from the Higgs Doublet. [4] . . . . . . . . 17

2.5 Vertex corrections to $\Gamma_{Z b b}$ from SUSY particles. [4] . . . . . . . 18

2.6 Example of gluon splitting. . . . . . . . . . . . . . 18

2.7 Fraction $e^{+} e^{-} \rightarrow u \bar{u}, d \bar{d}$ or $s \bar{s}$ which produce $b \bar{b}$ pairs versus the center of mass energy from the leading order matrix element (dashed) and resummation of logarithmic terms (solid) calculations. The fine-dotted curves correspond to variation of the mass by $5 \%$ and the dotted curves correspond to varying $\Lambda$ by a factor of $2 .[19] \ldots \ldots \ldots$

2.8 Fraction $e^{+} e^{-} \rightarrow u \bar{u}, d \bar{d}$ or $s \bar{s}$ which produce $b \bar{b}$ pairs versus the center of mass energy for three different Monte Carlos ( HERWIG (circles), JETSET (squares), and ARIADNE (crosses) ).[19] . . . . . . . . . 20

3.1 The SLAC Linear Collider. . . . . . . . . . . . . . . . 22 
3.2 The electron source. . . . . . . . . . . . . . . 23

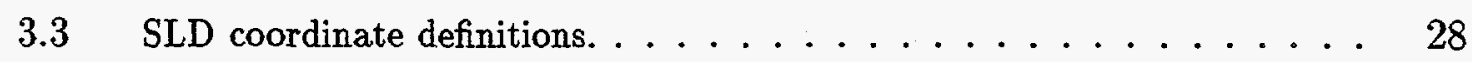

3.4 A section of the CDC endplate. . . . . . . . . . . . . 29

3.5 The left figure shows lines(bold) of constant potential and lines(thin) of constant field strength within a CDC cell. The right figure shows the drift path of charges caused by the passing of a track through the cell. . . . 3 31

3.6 CDC hit $z$ position from charge asymmetry versus fitted $z$ position of hit. $\quad 32$

$3.7 \quad \mathrm{CDC}$ hit finding efficiency versus layer number. . . . . . . . . 32

3.8 A slightly tilted view of the CDC sense wires showing the layout of the stereo wire planes. Only half of the wires are shown for clarity. . . . . 33

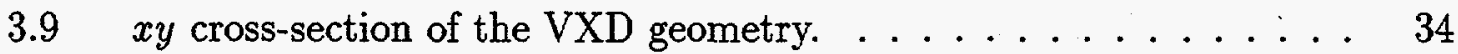

3.10 A $y z$ view of the VXD(top) and an event showing the VXD CCDs and pixel clusters on fitted tracks in the $x y$ view(bottom-left) and $r z$ view(bottom-right). . . . . . . . . . . . 36

3.11 The VXD triplet miss distance. . . . . . . . . . . . . 37

3.12 An SLD event showing tracks identified in the CDC and the associated energy deposits measured in the LAC. . . . . . . . . . . . . 39

3.13 Electromagnetic and hadronic modules of the LAC barrel. . . . . 40

3.14 Luminosity versus time for all SLD runs. . . . . . . . . . . . 41

3.15 Plots of the distance of closest approach of the track hits to the helix describing the track. The left plot shows the increasing uncertainty in this distance as one moves away from the sense wire (or cell) plane. The uncertainty is $\sim 120 \mu \mathrm{m}$ on average for tracks with momentum $>5 \mathrm{GeV} / c .43$

$3.16 r \phi$ and 3-D impact parameters for CDC alone tracks from $\mu^{+} \mu^{-}$events. $\quad 47$

3.17 Dip angle resolution and momentum resolution from $\mathrm{CDC}$ alone tracks in

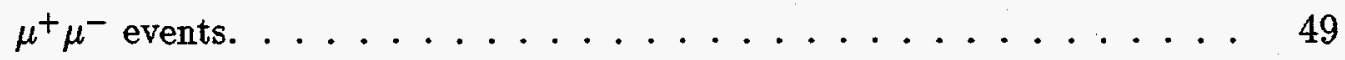

$3.18 r \phi$ and 3-D impact parameters for CDC+VXD tracks from $\mu^{+} \mu^{-}$events. $\quad 50$

3.19 Dip angle resolution and momentum resolution for CDC+VXD tracks

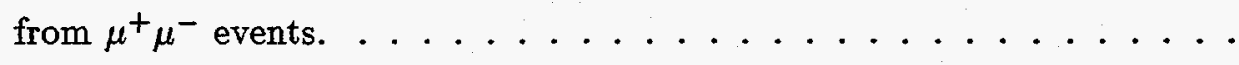

3.20 Distributions of variables indicative of the tracking performance for the CDC. . . . . . . . . . . . . . . . 
3.21 Distributions of variables indicative of the tracking performance for the

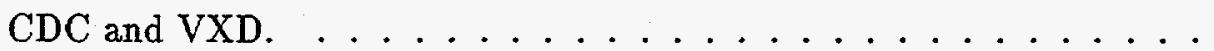

4.1 Beam positions for the 1994 run as determined from the currents applied to the corrector magnets, located near the interaction point, to keep the $e^{+} e^{-}$beams in collision. . . . . . . . . . . . . . . .

$4.2 x y$ impact parameters of tracks in $\mu$ pair events with respect to the measured primary vertex position determined independently by hadronic $Z^{0}$

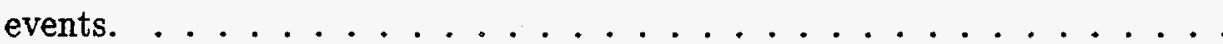

5.1 Drawing illustrating the signing of the impact parameters. Tracks which appear to have come from decays in the direction opposite to the associated jet are assigned negative impact parameters. Tracks from decays of particles with long lifetimes should have positive impact parameters. . . 60

5.2 Fraction of $B$-decay tracks correctly signed versus $y_{c u t}$. . . . . . . . 61

5.3 Miss distance of tracks in $\mu^{+} \mu^{-}$events. . . . . . . . . . . . 64

$5.4 r \phi($ solid $)$ and $r z$ (dashed) impact parameter resolutions vs. momentum for tracks at $\cos \theta=90^{\circ} \ldots \ldots \ldots 66$

5.5 The 2-D $r \phi$ impact parameter $(\delta), 2-\mathrm{D} r \phi$ normalized impact parameter $\left(\delta / \sigma_{\text {delta }}\right)$ and 2-D $r \phi$ impact parameter uncertainty $\left(\sigma_{\text {delta }}\right)$. The data is represented by the points and the Monte Carlo by the histogram. . . . . . . . 67

5.6 The 2-D $r z$ impact parameter $(\delta), 2-\mathrm{D} r z$ normalized impact parameter $\left(\delta / \sigma_{\text {delta }}\right)$. The data is presented the points and the Monte Carlo by the histogram. 68

5.7 The signing of $\chi \ldots \ldots \ldots \ldots 71$

5.8 Data and Monte Carlo distributions of the tagging variable $\chi \ldots \ldots 71$

6.1 Spectrum of lepton momentum from $B$ decays compared between the Monte Carlo simulation and CLEO data.[34] . . . . . . . . . . . 74

6.2 Spectra of different species of $D$ meson produced in $B$ hadron decays.[34] 75

6.3 Recent $B$ meson lifetime measurements from LEP(Moriond 1995). . . . 76

6.4 Recent $B$ baryon lifetime measurements from LEP(Moriond 1995). . . . 76

6.5 Peterson fragmentation distribution for $b$ and $c$ quarks. . . . . . . . 78

7.1 Distributions to which cuts are applied to select a pure hadronic event sample. The Monte Carlo is the line and data are the markers. . . . . . 81 
7.2 Number of tracks per event failing each track selection cuts for the CDC track alone and the CDC+VXD track. The last two bins show the number of tracks that pass the selection before and after efficiency corrections have been applied. .................... 85

7.3 Hit efficiency versus drift distance in the CDC. . . . . . . . . 86

7.4 Efficiency corrections applied to the Monte Carlo for quality CDC tracks. 87

7.5 Efficiency corrections applied to the Monte Carlo for the rate that quality CDC tracks link to VXD hits. . . . . . . . . . . . . . .

7.6 Distribution of the impact parameter divided by its uncertainty showing the accurate simulation of the resolution from the Monte Carlo. . . . . .

7.7 $\quad \chi^{2}$ of the fit of the data and Monte Carlo impact distributions versus width of the Gaussian used to degrade the resolution in the Monte Carlo and the frequency with which it is applied. . . . . . . . . . . .

8.1 $r \phi$ normalized impact parameters for $b, c, u d s$, and all Monte Carlo and data events. . . . . . . . . . . . . . . . . . .

8.2 Statistical uncertainty on $R_{b}$ for a fiducial data sample of $21 \mathrm{k}$ events versus $\epsilon_{b}$ and $\Pi_{b}$.The curves for $\Pi_{b}<R_{b}$ are unphysical since they correspond to anti-tagging $b$ events. With no tag $\Pi_{b}=R_{b} \ldots \ldots \ldots \ldots$

8.3 Normalized impact parameter distribution for Monte Carlo and data after resolution and efficiency corrections have been applied. . . . . . . 96

8.4 Plot of the tagging efficiency versus purity for $b$-quark events. . . . . . . 98

8.5 Plot of the number of tracks per event with significance $>3 \sigma . \ldots 99$

8.6 Variation in $R_{b}$ result with changes in tagging cuts. . . . . . . . 100

8.7 Plot of the contributions to the uncertainty in $R_{b}$ versus the significance cut at a multiplicity cut of $3 \ldots \ldots \ldots 10 . \ldots \ldots$

9.1 Distributions of the 3-D significance variable $\chi$ for $b, c$ and $u d s$ flavored

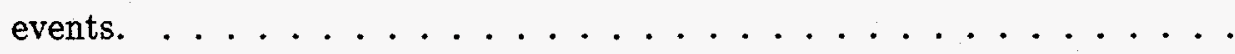

9.2 The distribution of the energy in the first two jets of the events relative to the total visible energy and the dot product of the quark and jet direction as a function of this. 
9.3 Indicators of accuracy to which the $B$-hadron direction is determined versus different jet finding and jet count cuts. . . . . . . . . . . . 114

9.4 The distance along the jet axis to the point of closest approach between the track and the jet versus the distance of closest approach between the track and jet normalized by the uncertainty on the track position along the d.o.c.a. direction. This is shown for long lived particles (i.e. $K_{s}^{0}, \Lambda, \Sigma$ decay products etc...) which contaminated the signal and for tracks from the secondary $b$ vertex only which contain the desired tagging information. 115

9.5 Negative $\chi$ distribution for tracks in $u d s$ events overlayed with the fit of the resolution function. . . . . . . . . . . . . . 117

9.6 Distribution of tracks probabilities for consistency with coming from the primary vertex for $\mathrm{b}, \mathrm{c}$ and $u d s$ events. . . . . . . . . . . . . . 118

9.7 Distribution of hemisphere probabilities for $b, c$ and $u d s$ flavored events. 119

9.8 Comparison of the 3-D significance variable $\chi$ between data and Monte Carlo for tracks whose point of closest approach to the jet access is behind the primary vertex. Efficiency and minor resolution corrections have been applied. . . . . . . . . . . . . . . . 120

9.9 Log of number of events versus probability for each hemisphere to be consistent with the primary vertex. . . . . . . . . . . 123

9.10 Statistical uncertainties in $R_{b}$.................. 124

9.11 Statistical uncertainties in $\epsilon_{b} \ldots \ldots \ldots 124$

9.12 Tag performance comparison between SLD and ALEPH. . . . . . 126

$9.13 b$-tag dependence on hemisphere probability cut. . . . . . . . . 127

$9.14 R_{b}$ from the lifetime probability tag versus the hemisphere probability cut. 128

9.15 Physics contributions to the uncertainty in $R_{b}$ versus the hemisphere probability cut. . . . . . . . . . . . . . . . 129

9.16 Uncertainties in $R_{b}$ from efficiency and smearing corrections. . . . . . 130

9.17 Uncertainties in $\epsilon_{c}$ from efficiency and smearing corrections. . . . . . . 130

9.18 Uncertainties in $R_{b}$ from efficiency corrections. . . . . . . . . . . . 131

9.19 Distribution of the significance variable $\chi$ versus the azimuthal angle of the detector. . . . . . . . . . . . . . . . . 133 
9.20 Distribution of the significance variable $\chi$ versus the polar angle of the detector. ......................... 133

9.21 Average $\chi$ and track counts for the 1993 and 1994 runs. . . . . . . . 134

9.22 b-hemisphere correlation components. . . . . . . . . . 136

9.23 Distributions used to determine component of the correlation from the polar angles of the $B$-hadrons. . . . . . . . . . . 137

9.24 Distributions used to determine primary vertex $z$ correlation component. 138

9.25 Correlation components estimated from the data. . . . . . . . . . . 139

9.26 Correlation components estimated from the Monte Carlo. . . . . . . . . 139

9.27 Contributions to the uncertainty in $R_{b}$ versus the hemisphere probability cut. ........................... 141

10.1 SLD $R_{b}$ measurements. . . . . . . . . . . . . . . . 145

10.2 Theoretical curves for $R_{b}$ dependence on the top mass with the SLD measurement result added. . . . . . . . . . . . . . 146

A.1 Mass distributions of identified $V^{0}$ s. The histogram represents the Monte Carlo and the points represent the data. . . . . . . . . . . . . 148

A.2 Gamma conversions rates distance from beam-line for the data(points) and Monte Carlo(histogram). . . . . . . . . . . . . . 150

A.3 Scatter plot of density of gamma conversion found in the region of the beam pipe to the inner wall of the CDC . . . . . . . . . . . . 151

A.4 Projections of the gamma conversion position distribution. . . . . . 153

B.1 Distributions of tagging variable and the resulting $b$-tagging performance for event tags used at SLD . . . . . . . . . . . 155

B.2 Illustration of the impact parameter sum method where the $b, c$ and $u d s$ distributions have been fit to the data to measure the branching fractions. . . . . . . . . . . . . . . 157

C.1 Demonstration of the strong linear dependence of the drift velocity on the pressure of the gas. . . . . . . . . . . . . . 159 
C.2 The comparison of the measured drift velocity to the calculated drift velocity shows that a simple linear equation with inputs of the monitored chamber pressure, water content of the gas, and temperature can accurately determine the correction. . . . . . . . . . . . 160

C.3 Dependence of drift velocity correction on water content of the CDC gas. 161

C.4 The drift velocity correction versus time. The dip corresponds to a time when the water content was maladjusted and indicates the strong dependence of the drift velocity on this. . . . . . . . . . . . . . 162

D.1 Variation of linking rate versus noise counts in the calorimeter, CDC and VXD for the Monte Carlo and data. . . . . . . . . . . . 165 


\section{Chapter 1}

\section{Introduction}

Through precision studies of the $Z^{0} \rightarrow b \bar{b}$ vertex it is hoped that stringent tests of the current theory of particle interactions (the Standard Model) may be made. Since the $b$-quark exists in a doublet with the top quark, there is a large coupling between these particles. This leads to significant top mass dependent corrections to the cross-section for $Z^{0} \rightarrow b \bar{b}$. In this cross-section, the vertex corrections are obscured by oblique corrections. The vertex corrections may be isolated by taking the ratio of the $Z^{0} \rightarrow b \bar{b}$ cross-section to other cross-sections for hadronic decays of the $Z^{0}$. In these ratios the oblique corrections mostly cancel while the vertex corrections remain. Experimentally, the best ratio to use is $\Gamma\left(Z^{0} \rightarrow b \bar{b}\right) / \Gamma\left(Z^{0} \rightarrow\right.$ hadrons $)$. Using the current top mass measurements from $\operatorname{CDF}\left(176 \pm 8 \pm 10 \mathrm{GeV} / c^{2}\right)[1]$ and $\mathrm{D} 0\left(199_{-21}^{+19} \pm 22 \mathrm{GeV} / c^{2}\right)[2]$ one expects a change of $\sim-1$ to $-2 \%$ in $R_{b}$ from the massless top quark value of $0.2193[3][4]$. As will be described in Sect. 2.1.7, there exists the possibility of observing evidence for new physics such as supersymmetric particles due to their presence in the vertex corrections[4][5]. LEP has made measurements of $R_{b}$ using several techniques and their current average is $1.8 \sigma$ high compared to the Standard Model[3]. Unless a problem in the experimental techniques is discovered, this may be one of the first indicators of physics beyond the Standard Model.

The analyses of this thesis use data collected from the observation of interactions resulting from the collision of an $e^{+}$and $e^{-}$beam. The beams are produced by the SLAC Linear Collider which accelerates and guides them to the collision point inside 
the SLAC Large Detector. The data represents the interaction of the collision decay products with the detector elements. The measurement of $R_{b}$ from this data is the subject of this thesis. The outline of the thesis is as follows: First, the motivation and theory are discussed. Next, the accelerator, detector, and processing of the data is described. Then, the analyses which use several techniques to tag decays involving the $b$-quark are detailed and the results given. Finally, it concludes with the implications of the results and prospects for future improvement of the measurement.

\section{LEP $R_{b}$ Measurements}

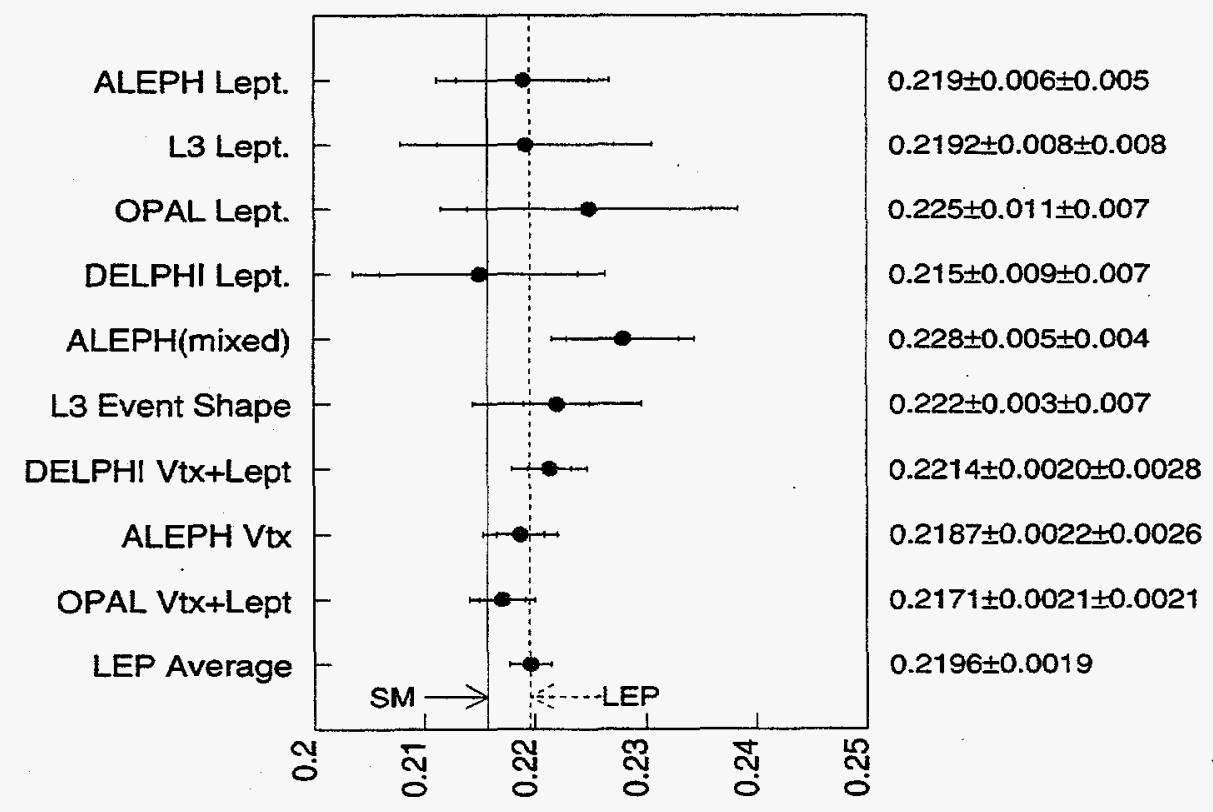

Figure 1.1: LEP $R_{b}$ Measurements from Moriond '95 Electroweak Conference. Uncertainty from $R_{c}$ not included. 


\subsection{Motivation for Performing Measurement using the SLD}

It is necessary to produce the purest possible sample of tagged $b$-decays to reduce the systematic uncertainties arising from the lack of knowledge of the $c$ and $u d s$ decay properties. Particles containing a $b$-quark are characterized by long lifetimes $(\sim 1.5 \mathrm{ps})$ and large mass $\left(\sim 5 \mathrm{GeV} / c^{2}\right)$. This results in decays of particles containing a $b$ quark that have high multiplicities of secondary vertices located far from the $e^{+} e^{-}$ interaction point. An example of a real data event which illustrates this is shown in Fig. 1.2. This is an event which satisfied a hard $b$-tag used in this thesis. To exploit the $b$-decay properties the experimental apparatus must be able to:

- locate the $e^{+} e^{-}$interaction position to high precision

- identify particles from decays of the $b$-quark as having come from a secondary vertex

Currently the most precise device for studying vertices in $Z^{0}$ decays is the pixel vertex detector(VXD) of the SLD. The very precise position measurements from the SLD and the small size and stability of the SLC beams allow the goals itemized above to be well achieved. The analyses presented here exploit the fact that the flight path resolution $(\sim 100 \mu \mathrm{m})$ is significantly smaller than the average decay length of a $b$ $\left(L=\beta \gamma c \tau=\left(\frac{p}{m}\right)(c \tau)=3 \mathrm{~mm}\right)$. The elements of the SLD which allow these precision measurements to be made will be discussed in detail in Chapter 3 .

\subsection{Methods by which $R_{b}$ is Measured}

All of the methods currently being used for measuring $R_{b}$ attempt to minimize the contamination of the tagged $b$-decay sample while maintaining a high $b$-tagging efficiency. The methods differ in what properties of the events or hemispheres are used in the $b$-tag. Some measurements have used global event shape variables such as the thrust and sphericity of an event[6]. Due to the large mass of the $b$ relative to 


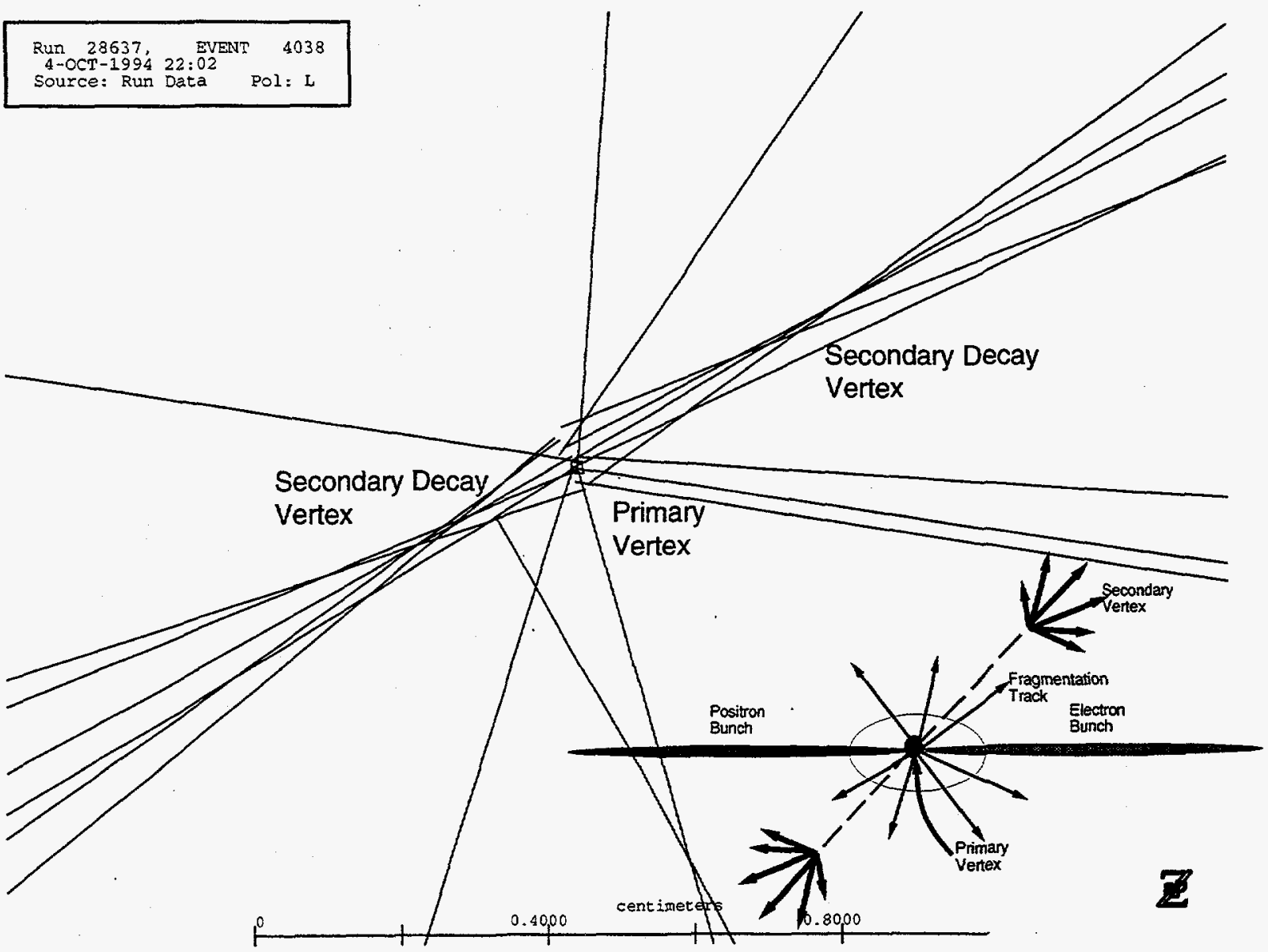

Figure 1.2: An SLD $Z^{0}$ event showing reconstructed charged tracks forming two distinct secondary vertices. This is likely a $Z^{0} \rightarrow b \bar{b}$ event.

any other quark but the top, the decays of b's may be distinguished by atypical event shapes resulting from the high transverse momentum of the decay products with respect to the original $b$ direction. These, measurements are very dependent on the fragmentation simulation of the Monte Carlo. Another, method uses high $\mathbf{p}$ and $\mathbf{p}_{t}$ leptons to $\operatorname{tag} b$-decays[7]. When a $b$-hadron decays with the production of a lepton, that lepton is likely to have a high total momentum and high momentum transverse to the $b$ direction, due to the large difference in mass between the $b$ and $c$ quarks. These leptons can then be identified and used as signatures of a $b$-decay. However, one must then correct for the imprecise semileptonic branching fraction of the $B$. Another class of tags are based on the characterization of $b$-decays as having a higher 
multiplicity[9] of tracks from secondary vertices significantly displaced from the $e^{+} e^{-}$ interaction point. These tags use the large miss distance of these $b$-decay tracks to the $e^{+} e^{-}$interaction point. These are called impact parameter tags[8]. The impact parameter is this miss distance or in words which are more common in this type of analysis, it is the distance of closest approach between the trajectory(track) and the interaction point. Both of the analyses presented here use this type of tag.

\section{3 b-Tags used in this Thesis}

The first method used in this thesis, the 2-D Impact Parameter Event Tag, tags the whole $b \bar{b}$ event using the count of large impact parameter tracks. It has the advantage of being a simple technique but its dependence on the Monte Carlo to estimate tagging rates results in significant systematics from the current uncertainties in the properties of $b$-decays. The second method, called the Lifetime Probability Tag, uses impact parameters of tracks to determine a probability that a collection of tracks in a hemisphere are consistent with coming from the interaction point to tag each $b$-decay separately. It makes minimal use of the Monte Carlo as the $b$-tagging rate is measured from the data. This significantly reduces the dependence on the simulation of the decays.

Historically, the event tag was one of the first measurements to make use of the SLD vertex detector. It was through this measurement that an understanding of the detector was obtained and checks of the Monte Carlo were made. It established the framework with which the higher precision measurement of $R_{b}$ using the probability tag was made. 


\section{Chapter 2}

\section{Theory}

\subsection{The Standard Model}

The Standard Model, which combines the theory of electroweak interactions, as given by the Weinberg-Salam model, with the theory of quantum chromodynamics has presently withstood all experimental tests[10]. It combines the forces through the gauge group representation: $S U(3) \otimes S U(2) \otimes U(1)$ where $S U(3)$ represents the gauge field of color, and $S U(2) \otimes U(1)$ represents the electromagnetic and weak interactions. It appears to accurately describe all fundamental forces except gravity. However, it is unsatisfactory in that it fails to predict the particle masses and the coupling constants[12]. Other potentially more satisfactory models exhibit deviations in the predicted behavior of vertex corrections that can be studied through precision electroweak measurements. One of the measurements which is most directly a measure of the vertex corrections is $R_{b}$.

Presented in this chapter is a brief introduction to the current understanding of the Standard Model and how $R_{b}$ is predicted in this framework. Furthermore, the corrections arising from the presence of top and possible corrections from the existence of SUSY particles are discussed. Uncertainties in the theoretical predictions are also stated. 


\subsubsection{Fundamental Particles}

In the Standard Model there are two sets of fundamental fermion particles. One set, the leptons, consists of three pairs of electromagnetically interacting particles. The first pair consists of the electron $(e)$, with charge -1 and spin $-1 / 2$, and its neutral partner the electron neutrino $\left(\nu_{e}\right)$ with spin $1 / 2$. The other pairs which contain the muon $(\mu)$ and $\tan (\tau)$ along with their neutral partners are identical in structure to the $e^{-}$pair except for their masses. The other set of fundamental particles are the quarks. Like the leptons, these particles are grouped in pairs. Each of the three pairs consists of a particle of charge $+2 / 3$ and spin $1 / 2$ and its partner with charge $-1 / 3$ and spin $-1 / 2$. The most notable difference between the pairs are the masses of the quarks. The particle pairs exist in left handed doublets

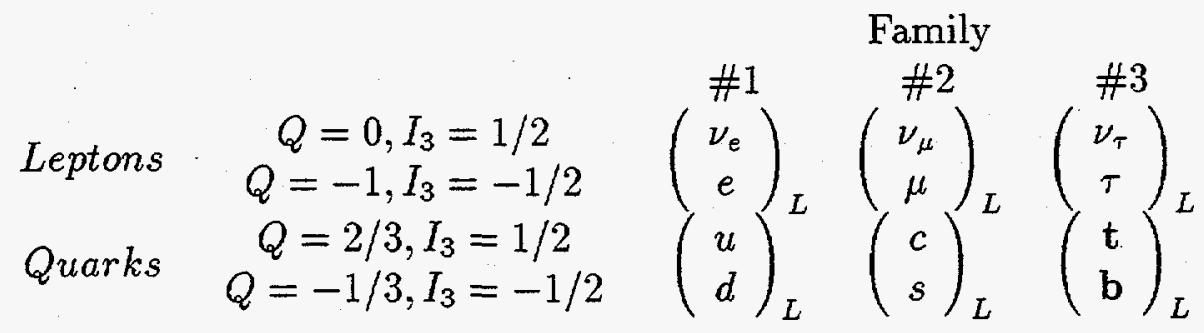

Table 2.1: Fundamental particle doublets.

and right handed singlets

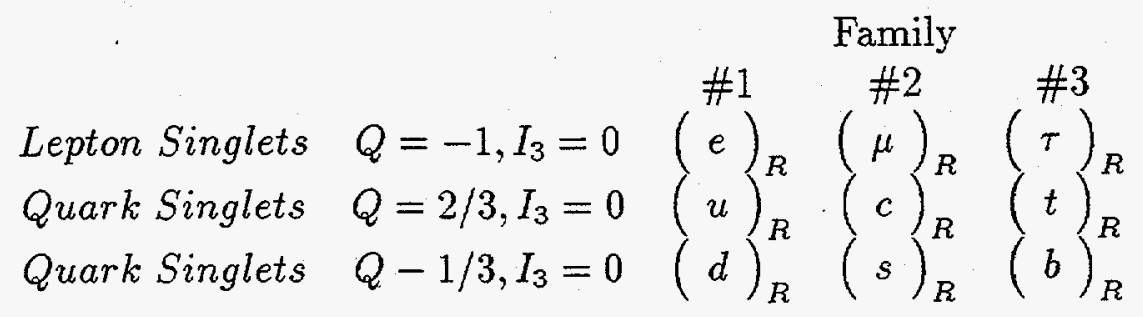

Table 2.2: Fundamental particle singlets.

All particles in these groups have now been observed. Ordered by mass, each set of corresponding left handed doublets and right handed singlets forms a family (i.e. first 
family $\left.=\left(\begin{array}{c}\nu_{e} \\ e\end{array}\right)_{L},\left(\begin{array}{l}u \\ d\end{array}\right)_{L}, e_{R}, u_{R}, d_{r}\right)$. The last fundamental particle to be observed was the top quark. First evidence of the top quark was announced in 1994 by CDF. More significant evidence for the top from both CDF and D0 were presented at the beginning of $1995[1][2]$.

\subsubsection{Gauge Bosons}

The Standard Model includes a set of gauge bosons for mediating the interaction between these particles. The observed carriers of the forces are the massless photon for the electromagnetic interaction, the very massive $W^{ \pm}$and $Z^{0}$ particles for the weak interaction, and the massless carrier of color, the gluon, for the strong interaction. The eigenstates for the electroweak bosons are the $W^{ \pm}, W_{3}$ and $B$. However, the observable neutral bosons (the $Z^{0}$ and photon) are a mixture of the $W_{3}$ and $B$ eigenstates.

$$
\begin{gathered}
S U(2) \text { Triplet of Gauge Bosons }\left(\begin{array}{c}
W^{+} \\
W_{3} \\
W^{-}
\end{array}\right) \\
S U(2) \text { Singlet Gauge Boson }(B) \\
S U(2) \text { Higgs Boson }\left(\begin{array}{c}
\phi^{+} \\
\phi^{0}
\end{array}\right)
\end{gathered}
$$

The eigenstates are combined through inclusion of the weak hypercharge $Y=2(Q-$ $I_{3}$ ) which is necessary to account for the particle charges. The mixing of the states is determined by the Weinberg angle as shown in Eqn. 2.1 and 2.2.

$$
\begin{aligned}
& A=B \cos \theta_{W}+W_{3} \sin \theta_{W} \\
& Z^{0}=B \sin \theta_{W}-W_{3} \cos \theta_{W}
\end{aligned}
$$

The value of the Weinberg angle relates the electromagnetc coupling strength ( $\alpha=$ $\left.\frac{e^{2}}{4 \pi \bar{h}_{c}}\right)$ and the weak coupling strength $\left(G_{F}=\frac{\sqrt{2} g^{2}}{8 M_{W}^{2}}\right)$ through the relation $\sin \theta_{W}=e / g$. 
This is given in terms of the weak gauge boson masses by $e / g=\sqrt{1-\left(M_{W} / M_{Z}\right)^{2}}$. The weak gauge bosons were first observed at CERN in 1982 by the UA1 and UA2 experiments. In 1989 LEP and SLAC simultaneously made precise measurements of the $Z^{0}$ width which through the simple proportion of the widths to the number of decay channels showed that there are no more than three families of particles.

\subsubsection{V-A Interaction}

In contrast to the electromagnetic interaction the weak interactions have different strengths for the coupling to left and right handed fermions. The $\mathrm{W}$ bosons only couple to left(right) handed fermions(anti-fermions). For the $\mathrm{Z}$ boson the form of the interaction is:

$$
\nu_{f}-a_{f} \gamma^{5}
$$

where $\nu$ is the vector coupling and $a$ is the the axial coupling for the $Z f f$ vertex. The values of these for the fundamental particles are:

\begin{tabular}{|l|c|c|}
\hline Particle & $\begin{array}{c}\text { Vector Coupling } \\
\nu_{f}=I_{3}^{f}-2 Q^{f} \sin ^{2} \theta_{w}\end{array}$ & $\begin{array}{c}\text { Axial Coupling } \\
a_{f}=I_{3}^{f}\end{array}$ \\
\hline$u, c, t$ & +0.19 & +0.5 \\
$d, s, b$ & -0.34 & -0.5 \\
$\nu_{e}, \nu_{\mu}, \nu_{\tau}$ & +0.50 & +0.5 \\
$e, \mu, \tau$ & -0.03 & -0.5 \\
\hline
\end{tabular}

Table 2.3: Vector and axial coupling strengths for the neutral current interaction.

\subsubsection{Strong Interactions and Hadrons}

In addition to the electroweak force the quarks also interact through the strong or color force. This governs how the quarks may combine to form observable particles other than the leptons. The color quantum numbers are red(r), green(g), blue(b) and the corresponding anticolors. Only combinations which are color singlets may be 
observed. This implies that the quarks are not observable. Only combinations such as $q \bar{q}$ (mesons) and $q q q$ (baryons) are observed. These combinations are formed during the fragmentation process.

Unlike the electromagnetic and weak forces, the strong force increases with increasing separation of the interacting particles. The potential responsible for this has the form $V \sim \lambda r$; where $\lambda$ is a constant and $r$ is the separation. This results in the infrared confinement of the quarks. At small separations the interaction of the color carrying gluons with themselves causes the force to decrease and approach what is called asymptotic freedom. In the case of the $e^{+} e^{-}$collisions occurring at the SLD, the quarks from the $Z$ decay have enough energy that they do not form a bound system such as the $\Upsilon$ states produced at CLEO. Instead, as the quarks move away from the decay point the color force increases until a lower energy state of two hadrons is produced.

\subsection{4 $B$-Hadrons and Their Decays}

Hadrons produced by fragmentation that have a $b$ or $\bar{b}$ in them are called $B$-hadrons. The lowest lying $B$-hadrons include $B^{ \pm}, B^{0}, B_{s}$, and the $B$-baryons $\left(\Lambda_{b}\right)$. Because the $b$ quark is the lighter partner of the third family it must decay weakly. The coupling between the mass eigenstates of the quarks is given by the Kobayashi-Maskawa matrix:

$$
\left(\begin{array}{c}
d^{\prime} \\
s^{\prime} \\
b^{\prime}
\end{array}\right)=\left(\begin{array}{lll}
V_{u d} & V_{u s} & \mathbf{V}_{\mathbf{u b}} \\
V_{c d} & V_{c s} & \mathbf{V}_{\mathbf{c b}} \\
V_{t d} & V_{c s} & \mathbf{V}_{\mathbf{t b}}
\end{array}\right)\left(\begin{array}{l}
d \\
s \\
b
\end{array}\right)
$$

The KM matrix is unitary. Thus, the observance that $V_{u b}$ and $V_{c b}$ are $<<1$. implies that $V_{t b} \approx 1$. Measurements of the $b$-asymmetry, $A_{b}=\frac{2 \nu_{b} a_{b}}{v_{b}^{2}+a_{b}^{2}}$, indicate that the weak isospin of the $b$ is $\approx \frac{1}{2}[11]$. This implies that the $b$ quarks must exists in an SU(2) doublet. Thus, before the CDF and D0 results, it was known that the top quark must exist. The strongest coupling of the $b$ to a lighter quark is $V_{c b}=0.045$. The decay time for $b \rightarrow W^{-} c$ is given by $\tau_{b} \approx \frac{192 \pi^{3}}{3 G_{f}^{2} M_{b}^{5} V_{c b}^{2}}$. For a $b$ mass of $5 \mathrm{GeV}$ this 
gives $1.5 \mathrm{ps}$ in the rest frame of the $b$. When boosted into the lab frame this results in the observed $\sim 3 \mathrm{~mm}$ decay length[13].

\subsection{5 $R_{b}$}
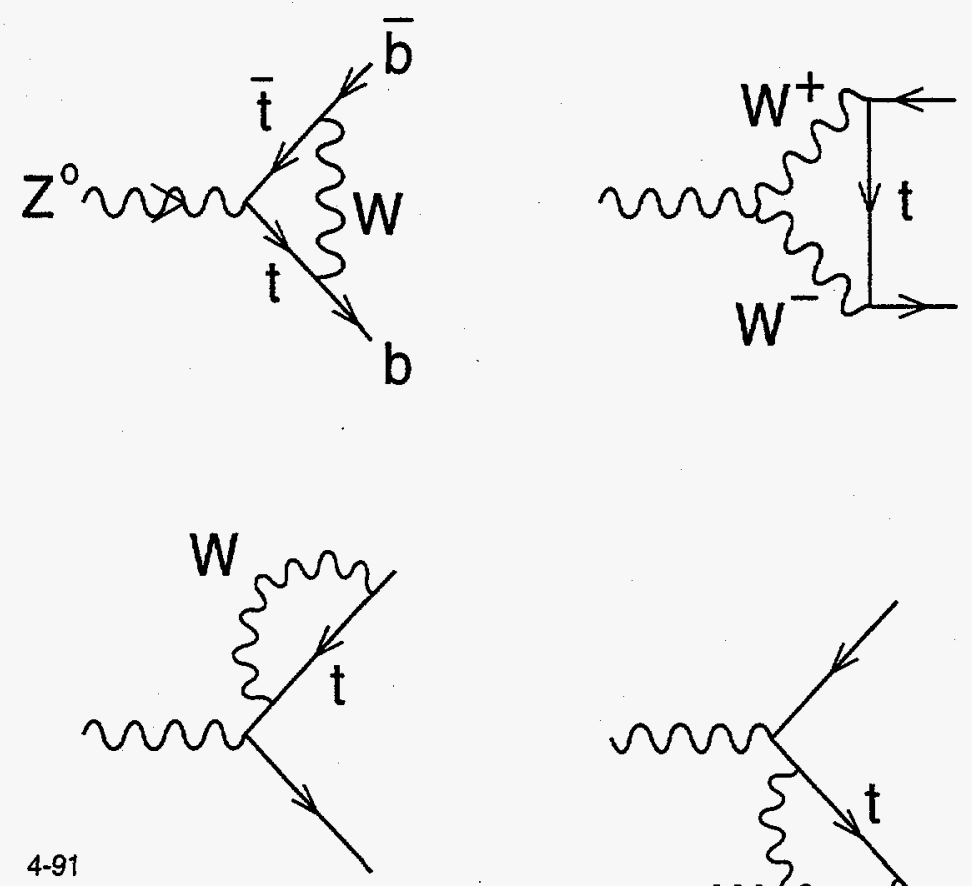

$6913 \mathrm{A1}$

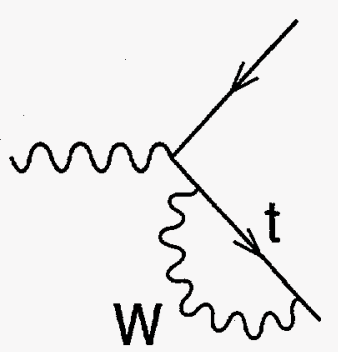

Figure 2.1: Vertex corrections to $\Gamma_{Z b b}$ from $t$ and $W$.

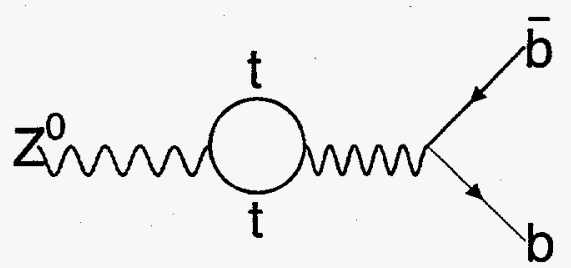

Figure 2.2: An oblique correction to $\sigma\left(Z^{0} \rightarrow b \bar{b}\right)$.

The top mass dependence of $R_{b}$ results from the corrections to the $Z^{0} \rightarrow b \bar{b}$ vertex shown in Fig. 2.1. Oblique corrections arising from diagrams like the self energy of the top shown in Fig. 2.2 make the desired vertex corrections to the $Z^{0} \rightarrow b \bar{b}$ width 
immeasurable. By taking the ratio $R_{b}=\frac{\Gamma\left(Z^{0} \rightarrow b \bar{b}\right)}{\Gamma\left(Z^{0} \rightarrow \text { hadrons }\right)}$ the problem is circumvented because the oblique corrections mostly cancel in this ratio exposing the vertex corrections from $W^{ \pm}, t$ and any new physics particles that can couple the $Z^{0}$ and $b$. Without correcting for the top mass the Standard Model predicts an $R_{b}$ of 0.2193. With an $m_{\text {top }} \approx 174 \mathrm{GeV} / c^{2}$ the predicted value is $0.2157 \pm 0.0006$ for a Higgs mass between $300 \mathrm{GeV}$ and $1000 \mathrm{GeV}[14]$. The current average from LEP is $0.2196 \pm 0.0019$ which is $\sim 2 \sigma$ from the Standard Model prediction[3][14].

The $e^{+} e^{-} \rightarrow Z^{0}, \gamma \rightarrow q \bar{q}$ width is given by the square of the sum of the $e^{+} e^{-} \rightarrow$ $\gamma \rightarrow q \bar{q}$ and $e^{+} e^{-} \rightarrow Z^{0} \rightarrow q \bar{q}$ matrix elements. The matrix element for the electromagnetic coupling is derived from the interaction of the particle current with the field:

$$
-i e\left(j^{e m}\right)^{\mu} A_{\mu}=-i e\left(\bar{\Psi} \gamma^{\mu} Q \Psi\right) A_{\mu}
$$

From this the Feynman factor for the vertices

$$
-i e Q_{f} \gamma^{\mu}
$$

and for the propagator

$$
-i g_{\mu \nu} / s
$$

are obtained. Likewise for the electroweak $Z q$ interaction one has the following current field interaction:

$$
\frac{-i g}{\cos \theta_{W}}\left(J_{\mu}^{N C}\right) Z^{\mu}=\frac{-i g}{\cos \theta_{W}}\left(J_{\mu}^{3}-\sin ^{2} \theta_{W} j_{\mu}^{e m}\right) Z^{\mu}
$$

from which the following factors are obtained:

$$
\begin{gathered}
\text { vertex factor }=\frac{-i g}{\cos \theta_{W}} \gamma^{\mu} 1 / 2\left(\nu_{f}-a_{f} \gamma^{5}\right) \\
\text { propagator factor }=\frac{i\left(-g^{\mu \nu}+k^{\mu} k^{\nu} / M_{Z}^{2}\right)}{s-M_{W}^{2}+i M_{Z} \Gamma_{Z}}
\end{gathered}
$$

These factors are then combined to give the matrix elements

$$
\mathcal{M}_{\gamma}=-e^{2}\left(\bar{q} \gamma^{\nu} q\right) \frac{1}{s}\left(\bar{e} \gamma_{\nu} e\right)
$$


and

$$
\mathcal{M}_{Z}=-\frac{g^{2}}{4 \cos ^{2} \theta_{W}}\left(\bar{q} \gamma^{\nu}\left(\nu_{q}-a_{q} \gamma^{5}\right) q\right) \frac{g_{\nu \mu}-k_{\nu} k_{\mu} / M_{Z}^{2}}{s-M_{Z}^{2}+i M_{Z} \Gamma_{Z}}\left(\bar{e} \gamma^{\mu}\left(\nu_{e}-a_{e} \gamma^{5}\right) e\right)
$$

Finally one obtains the differential cross-section for $Z q$ as follows

$$
\frac{d \sigma}{d \Omega}=\frac{1}{64 \pi^{2} s}\left|\mathcal{M}_{\gamma}+\mathcal{M}_{\mathcal{Z}}\right|^{2}
$$

Averaging over the initial spin states and integrating over the solid angle gives:

$$
\begin{array}{r}
\sigma\left(e^{+} e^{-} \rightarrow q \bar{q}\right)=\frac{4 \pi \alpha^{2}}{s} \times\left[Q_{q}^{2}+2 Q_{q} \operatorname{Re}\left(\frac{\sqrt{2} G M_{Z}^{2}}{s-M_{Z}^{2}+i M_{Z} \Gamma_{Z}} \times \frac{s}{e^{2}}\right) \nu_{q} \nu_{e}+\right. \\
\left.\left|\frac{\sqrt{2} G M_{Z}^{2}}{s-M_{Z}^{2}+i M_{Z} \Gamma_{Z}} \times \frac{s}{e^{2}}\right|^{2}\left(\nu_{q}^{2}+a_{q}^{2}\right)\left(\nu_{e}^{2}+a_{e}^{2}\right)\right]
\end{array}
$$

The definition of $R_{q}$ is

$$
R_{q}=\frac{\sigma\left(e^{+} e^{-} \rightarrow q \bar{q}\right)}{\sum_{i} \sigma\left(e^{+} e^{-} \rightarrow q_{i} \overline{q_{i}}\right)}
$$

which, at the $Z^{0}$ peak, where the $Z^{0}$ coupling effects (represented by the last term of Eqn. 2.4) dominate, has the following simple relation to the vector and axial couplings with values obtained using input from Table 2.3:

$$
\begin{aligned}
& R_{u, c, t}=\frac{\left(\nu_{u}^{2}+a_{u}^{2}\right)}{2 \times\left(\nu_{u}^{2}+a_{u}^{2}\right)+3 \times\left(\nu_{d}^{2}+a_{d}^{2}\right)}=0.171 \\
& R_{d, s, b}=\frac{\left(\nu_{d}^{2}+a_{d}^{2}\right)}{2 \times\left(\nu_{u}^{2}+a_{u}^{2}\right)+3 \times\left(\nu_{d}^{2}+a_{d}^{2}\right)}=0.2193
\end{aligned}
$$

\subsubsection{Top Quark Mass Dependence}

The interest in $R_{b}$ results from the vertex corrections which alter $R_{b}$ from the value in Eqn. 2.5. The top quark mass dependence of $R_{b}$ is explained by J. Bernabeu et al.[15]. They parameterize the width into fermions by:

$\Gamma\left(Z^{0} \rightarrow f \bar{f}\right)=\frac{N_{c}^{f}}{48} \frac{\hat{\alpha}}{\hat{s}_{W}^{2} \hat{c}_{W}^{2}} m_{Z} \sqrt{1-\left(\frac{4 m_{f}^{2}}{m_{Z}^{2}}\right)^{2}}\left(\left|2 a_{f}\right|^{2}\left(1-\frac{4 m_{f}^{2}}{m_{Z}^{2}}\right)+\left|2 \nu_{f}\right|^{2}\left(1+\frac{1}{2}\left(\frac{4 m_{f}^{2}}{m_{Z}^{2}}\right)^{2}\right)\right)$ 


$$
\times\left(1+\delta_{f}^{(0)}\right)\left(1+\delta_{Q E D}\right)\left(1+\delta_{Q C D}\right)\left(1+\delta_{\mu}^{f}\right)\left(1+\delta_{b}\right)_{b \bar{b} \text { only }}
$$

The factors $\hat{\alpha}$ and $\hat{s}_{W}^{2}$ are the values of the fine structure constant and Weinberg angle at the $M_{Z}$ scale. Oblique corrections resulting from the self energy diagrams of the top and Higgs have been absorbed into $\hat{s}_{W}^{2}$. They will be discussed further in Sect. 2.1.6.1. Both $\hat{\alpha}$ and $\hat{s}_{W}^{2}$ have a small dependence on the top mass that will cancel when the ratio of the cross-sections is taken. The $1+\delta_{f}^{(0)}$ factor in Eqn. 2.6 includes small $(\sim 0.5 \%)$ corrections for massless quark loops and gauge boson self energies. The $1+\delta_{Q E D}$ factor includes negligible $(\sim 0.02 \rightarrow 0.08 \%)$ final state QED corrections. QCD corrections resulting from gluon radiation are included in the $1+\delta_{Q C D}$ factor. The form of the QCD correction in terms of the strong coupling constant $\alpha_{s}$ is shown in Eqn. 2.7[17].

$$
1+\delta_{Q C D}=1+\frac{\alpha_{s}}{\pi}+1.41\left(\frac{\alpha_{s}}{\pi}\right)^{2}
$$

These corrections will cancel in the ratio $\left(R_{b}\right)$ leaving only the vertex corrections and contributions from the quark masses.

The last factor in Eqn. 2.6, $1+\delta_{b}$, contains all of the vertex corrections from the top mass and any new physics. For the Standard Model corrections this is:

$$
\delta_{b} \approx 10^{-2}\left(-\frac{1}{2} \frac{m_{t}^{2}}{m_{Z}^{2}}+\frac{1}{5}\right)
$$

Finally, after including the mass effects in each of the widths, the following result for $R_{b}$ is obtained:

$$
\begin{gathered}
R_{b}=\frac{\Gamma\left(Z^{0} \rightarrow b \bar{b}\right)}{\Gamma\left(Z^{0} \rightarrow \text { hadrons }\right)}=\frac{\Gamma_{b}}{\Gamma_{b}+\Gamma_{c}+2 \Gamma_{s}+\Gamma_{u}}=\left(1+\frac{\Gamma_{c}}{\Gamma_{b}}+2 \frac{\Gamma_{s}}{\Gamma_{b}}+\frac{\Gamma_{u}}{\Gamma_{b}}\right)^{-1} \\
=\left(1+\frac{\left(\frac{1}{2}\right)^{2}+\nu_{c}^{2}}{\left(\frac{1}{2}\right)^{2}+\nu_{b}^{2}} \frac{1}{0.9960\left(1+\delta_{b}\right)}+\frac{2}{0.9949\left(1+\delta_{b}\right)}+\frac{\left(\frac{1}{2}\right)^{2}+\nu_{u}^{2}}{\left(\frac{1}{2}\right)^{2}+\nu_{b}^{2}} \frac{1}{0.9955\left(1+\delta_{b}\right)}\right)^{-1}
\end{gathered}
$$

The behavior of the top mass dependence of $R_{b}$ is shown in Fig. 2.3. 


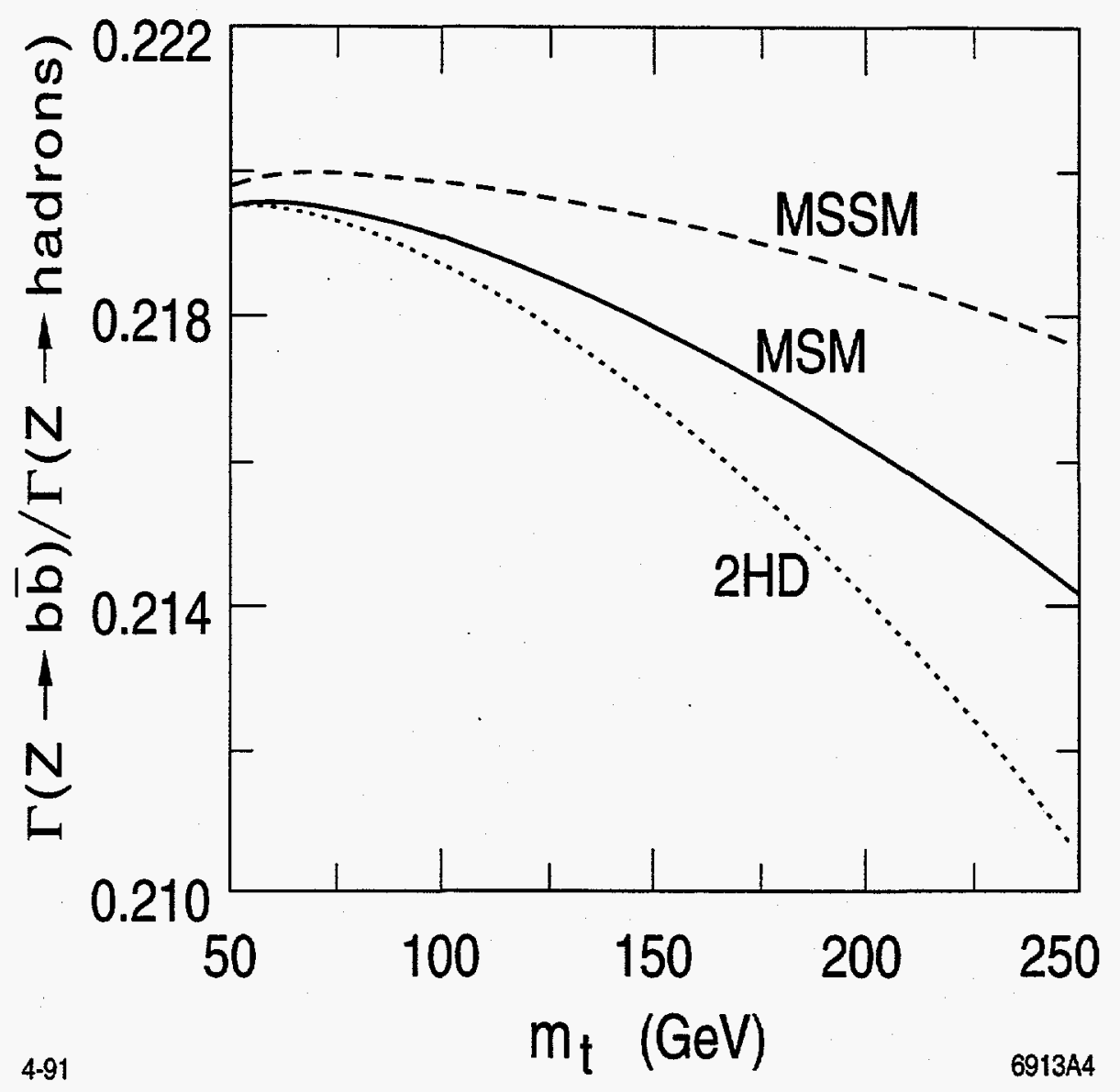

Figure 2.3: Top mass dependence of $R_{b}$ for the Standard Model(MSM), the Minimal Supersymmetric Extension of the Standard Model (MSSM), and the Standard Model with a second Higgs doublet. Corrections that have not been applied would lower the curves. The deviations between models remain unchanged when all corrections are applied.[4]

\subsubsection{Oblique Corrections}

The oblique corrections are worth further discussion as they are responsible for selecting $R_{b}$ instead of $\Gamma\left(Z^{0} \rightarrow b \bar{b}\right)$ as the desired value to be measured. Following the formalism of Verzegnassi et al.[16], $\Gamma\left(Z^{0} \rightarrow b \bar{b}\right)$ may be written in terms of its massless top value $\left(\Gamma_{b}^{(0)}\right)$, its vertex corrections $\left(\delta_{b}\right)$, and its oblique corrections $\left(\delta_{\rho}\right)$ as shown in Eqn. 2.9 .

$$
\Gamma_{b} \approx \Gamma_{b}^{(0)}\left(1+\delta_{b}+\delta_{\rho}\right)
$$




\begin{tabular}{|l|c|c|}
\hline Branching Fraction & Value for $m_{\text {top }}=0 \mathrm{GeV} / c^{2}$ & Value for $m_{\text {top }}=174 \mathrm{GeV} / c^{2}$ \\
\hline$R_{b}$ & 0.2193 & $0.2157 \pm 0.0004[14][15]$ \\
$R_{c}$ & 0.171 & 0.171 \\
$R_{u d s}$ & 0.610 & 0.613 \\
\hline
\end{tabular}

The values of the vertex and oblique corrections are given by Eqn. 2.10.

$$
\delta_{b} \approx-\frac{20}{13} \frac{\alpha}{\pi} \frac{m_{t}^{2}}{M_{Z}^{2}}-\frac{10}{3} \frac{\alpha}{\pi} \ln \frac{m_{t}^{2}}{M_{Z}^{2}}, \delta_{\rho} \approx \frac{19}{13} \frac{\alpha}{\pi} \frac{m_{t}^{2}}{M_{Z}^{2}}
$$

As mentioned earlier, these oblique corrections are due to loops in the $Z^{0}$ propagator resulting from the large top mass. In Eqn. 2.9 and 2.10 one sees that the first term of the oblique correction significantly cancels the vertex correction thus reducing the top mass dependence of the partial width. However, since the propagator corrections apply to all $\Gamma\left(Z^{0} \rightarrow q \bar{q}\right)$ and the vertex corrections only to $\Gamma\left(Z^{0} \rightarrow b \bar{b}\right)$ the influence of the oblique corrections is greatly reduced by taking the ratio of the partial widths for $b$ 's to any other hadronic width or, in the case of this thesis, the total hadronic width of the $Z^{0}$. The effect of the oblique corrections on $R_{b}$ is only $0.1 \%$ [4].

\subsubsection{Supersymmetric Extensions}

A model where all of the Standard Model particles have partners is the Supersymmetric Extension of the Standard Model(MSSM). In MSSM, for each fermion(boson) there is a corresponding supersymmetric boson(fermion). It has the promise of relating fermions and bosons in different representations thus making it possible for the theory to give a clear relation of the quarks to their forces[12]. The super-particle masses are currently believed to be too high to be observed with current accelerators but may be observed at the higher LEP energy expected in 1996. Several forms of supersymmetry theory have been proposed which alter the dependence of $R_{b}$ on the top quark mass. Among these are minimal supersymmetric models with very light super partners.

Michael Boulware and Donald Finnell[4] have calculated the behavior of $R_{b}$ versus the top mass for two non-Standard Models. First they recalculated the top mass 


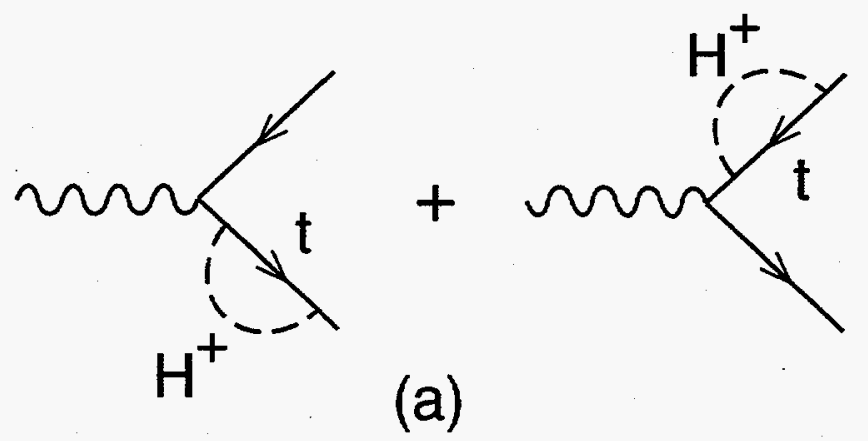

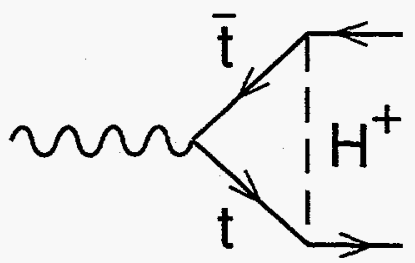

(b)

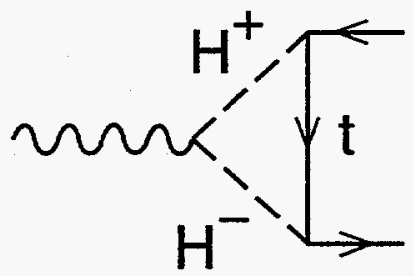

(c) $\quad 4-91$

Figure 2.4: Vertex corrections to $\Gamma_{Z b b}$ from the Higgs Doublet. [4]

dependence of $R_{b}$ with the addition of a second Higgs doublet. The second doublet is necessary for both the top and bottom quarks to have mass in the SUSY models. The additional vertex correction diagrams resulting from the $H^{ \pm}$particles formed from these doublets are shown in Fig. 2.4. This addition is found to significantly enhance the amount of correction versus $m_{t o p}$ (see Fig. 2.3). Finally, they reperform the calculation with the addition of the minimal SUSY particles which are present in the Minimal Supersymmetric extension of the Standard Model(MSSM). These sparticles include the charginos, higgsinos and winos. Examples of the new diagrams resulting from the existence of these particles are shown in Fig. 2.5. It is found that the main effect of MSSM is to increase the width thus reducing the relative magnitude of the radiative corrections from the charged Higgs and top. The result is shown in Fig. 2.3. The higher $R_{b}$ value predicted by the the MSSM is in good agreement with the current world average for $R_{b}$ [18][4]. It should be noted that the deviations from the MSM represent the largest attainable in the parameter space. 


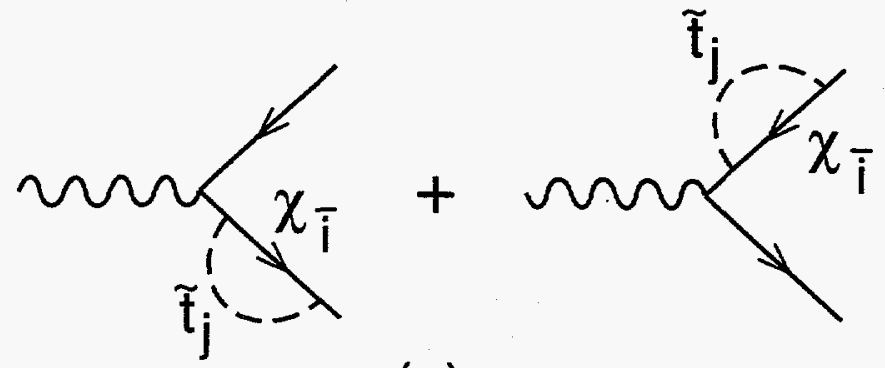

(a)

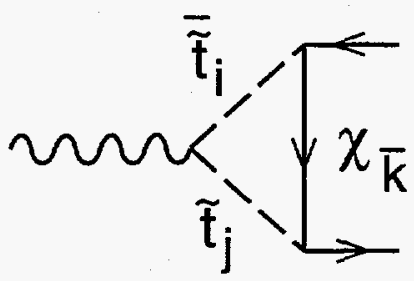

$6913 \mathrm{A3}$

(b)

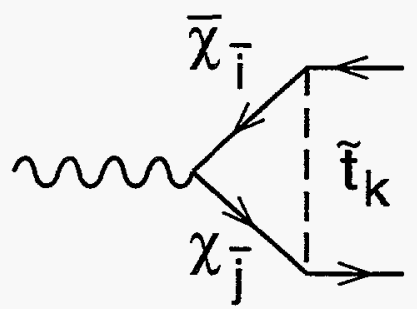

(C) $\quad 4-91$

Figure 2.5: Vertex corrections to $\Gamma_{Z b b}$ from SUSY particles. [4]

\subsection{Theoretical Uncertainties in $R_{b}$}

The largest theoretical uncertainty on $R_{b}$ comes from the lack of knowledge of the $b$-quark mass. The uncertainty is $\sim 5 \%$. This causes a large uncertainty in the "heavy quark pair multiplicity"[19] from gluon splitting in light quark events.

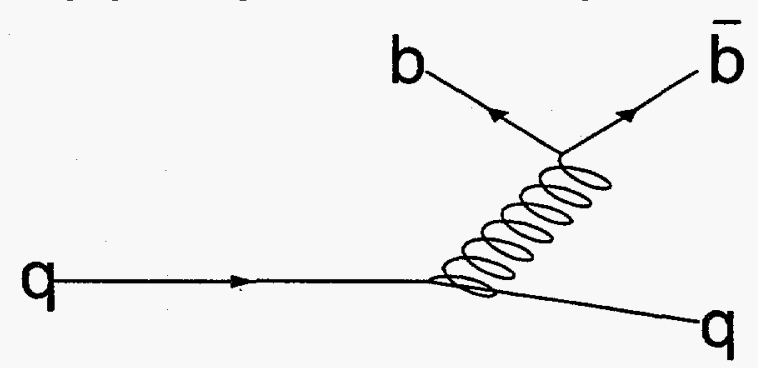

Figure 2.6: Example of gluon splitting. 


\subsubsection{Gluon Splitting}

Gluon splitting is where a quark line radiates a gluon which then produces a quark antiquark pair. This can lead to the production of a $b \bar{b}$ pair in light quark events thus altering the value of $R_{b}$. The rate for producing $b \bar{b}$ pairs in light quark events is strongly dependent on the poorly known $b$-quark mass $(5 \pm 0.25 \mathrm{GeV})$, the QCD scale parameter $(\Lambda)$ and procedure by which the large logarithmic terms in the calculation are summed. These effects are demonstrated in Fig. 2.7. The large uncertainty in the $b$-quark mass is due to the current inability to fully calculate the effects of confinement. Gluon splitting can cause a correction to $R_{b}$ by as much as $1 \%$ and is considered by some to be a likely cause of the current discrepancy between experiment and theory[19]. The $b \bar{b}$ production rate in light quark events for several simulation models is shown in Fig. 2.8. The simulation that will be used for the analyses of this thesis is JETSET.

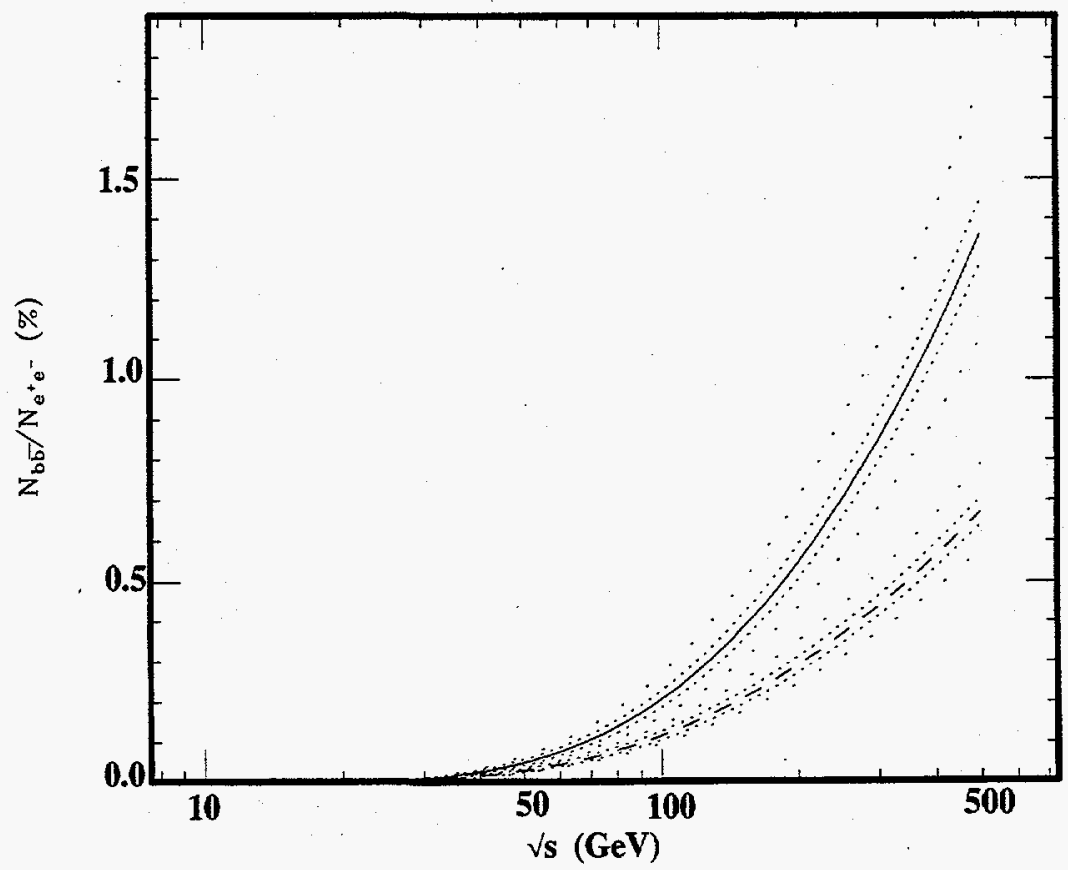

Figure 2.7: Fraction $e^{+} e^{-} \rightarrow u \bar{u}, d \bar{d}$ or $s \bar{s}$ which produce $b \bar{b}$ pairs versus the center of mass energy from the leading order matrix element (dashed) and resummation of logarithmic terms (solid) calculations. The fine-dotted curves correspond to variation of the mass by $5 \%$ and the dotted curves correspond to varying $\Lambda$ by a factor of 2.[19] 


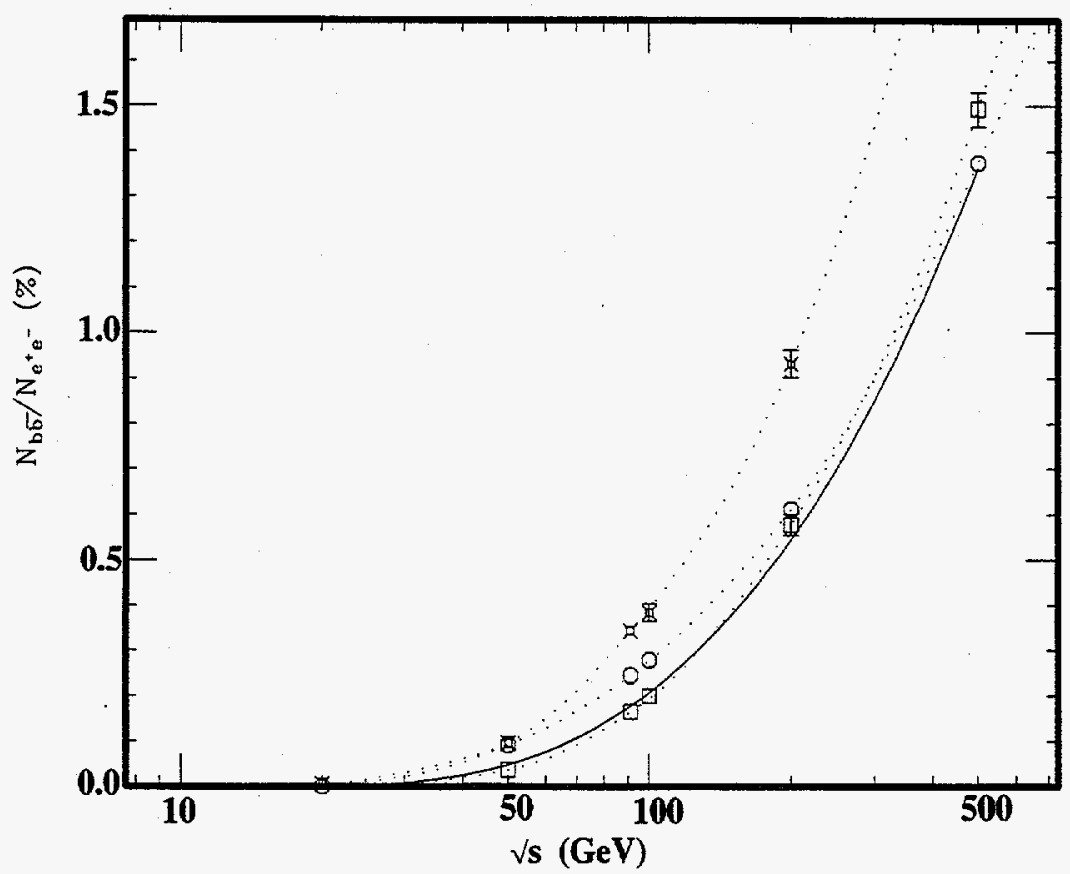

Figure 2.8: Fraction $e^{+} e^{-} \rightarrow u \bar{u}, d \bar{d}$ or $s \bar{s}$ which produce $b \bar{b}$ pairs versus the center of mass energy for three different Monte Carlos ( HERWIG (circles), JETSET (squares), and ARIADNE (crosses) ).[19] 


\section{Chapter 3}

\section{Experimental Apparatus and Detector Simulation}

The data used for the analyses presented in this thesis comes from readout of the SLAC Large Detector(SLD) for interactions produced by the collision of electron and positron bunches from the SLAC Linear Collider(SLC). The $3.2 \mathrm{~km}$ SLC is novel in that it achieved collision energies of $91.2 \mathrm{GeV}$ using a machine that was small compared to what previous technologies had required. Typically, storage rings for high energy beams are considerably larger as a large curvature radius is needed to reduce the energy loss of the particles through synchrotron radiation. An example of such an accelerator and storage ring is LEP whose circumference is $27 \mathrm{~km}$. SLC circumvents this costly problem by accelerating the particles in a single linear section (LINAC) and then bringing the electron and positron bunches around separate arcs rotating their momenta by $\pm 90^{\circ}$ and colliding them into each other inside the SLD. The SLD was designed for observing the products of the $Z^{0}$ decays. First, the aspects of the SLC and the SLD relevant to the analyses of this thesis are discussed in this chapter. Then, the simulation of the SLD and performance checks of the real SLD are described. 


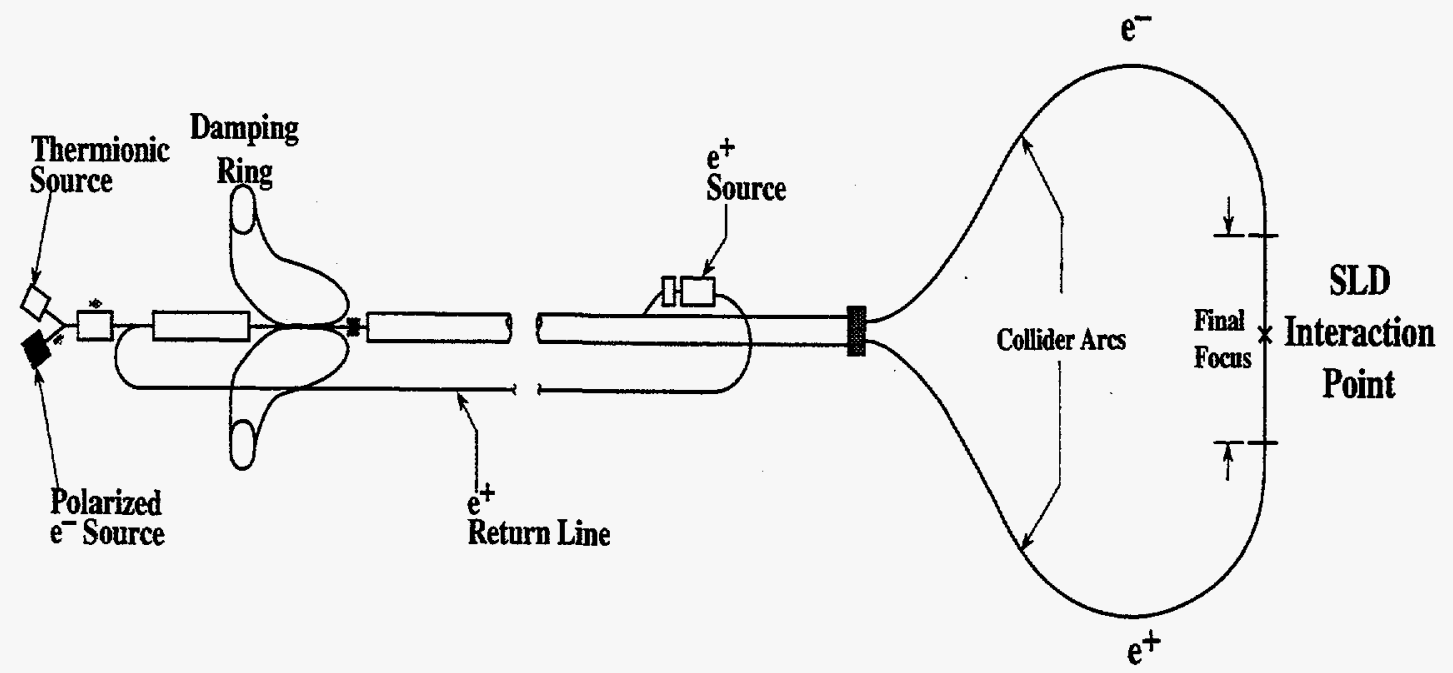

Figure 3.1: The SLAC Linear Collider.

\subsection{The SLAC Linear Collider}

The layout of the SLC is shown in Fig. 3.1. It consists of a $3 \mathrm{~km}$ long linear accelerator(LINAC) and arcs which bring the bunches of electrons and positrons into collision. The LINAC uses $\sim 24465 \mathrm{MWatt}$ klystrons[20] to accelerate the particles. The microwaves produced by the klystrons are guided by copper waveguides to the beam-pipe which is 25 feet below the surface. The microwaves create an alternating field in the cavities which are in phase with the passage of the electrons and positrons such that they will always experience an accelerating field. The electrons and positrons are accelerated to $46.6 \mathrm{GeV}$ by the time they reach the end of the LINAC. The width of the beam is maintained throughout the LINAC by quadrupole and sextupole magnets. Transverse tails on the bunches are also removed by use of collimators. These devices reduce the aperture for the beam and scrape away particles far away from the ideal orbit.

The beams are bunches of $\approx 3 \times 10^{10}$ particles. They are produced at a rate of $120 \mathrm{~Hz}$. Each bunch starts with the excitation of electrons from a strained GaAs cathode using two $2 n s$ pulses from an Nd:YAG-pumped Ti:sapphire laser[21]. A diagram of the setup is shown in Fig. 3.2. The two bunches produced are accelerated 


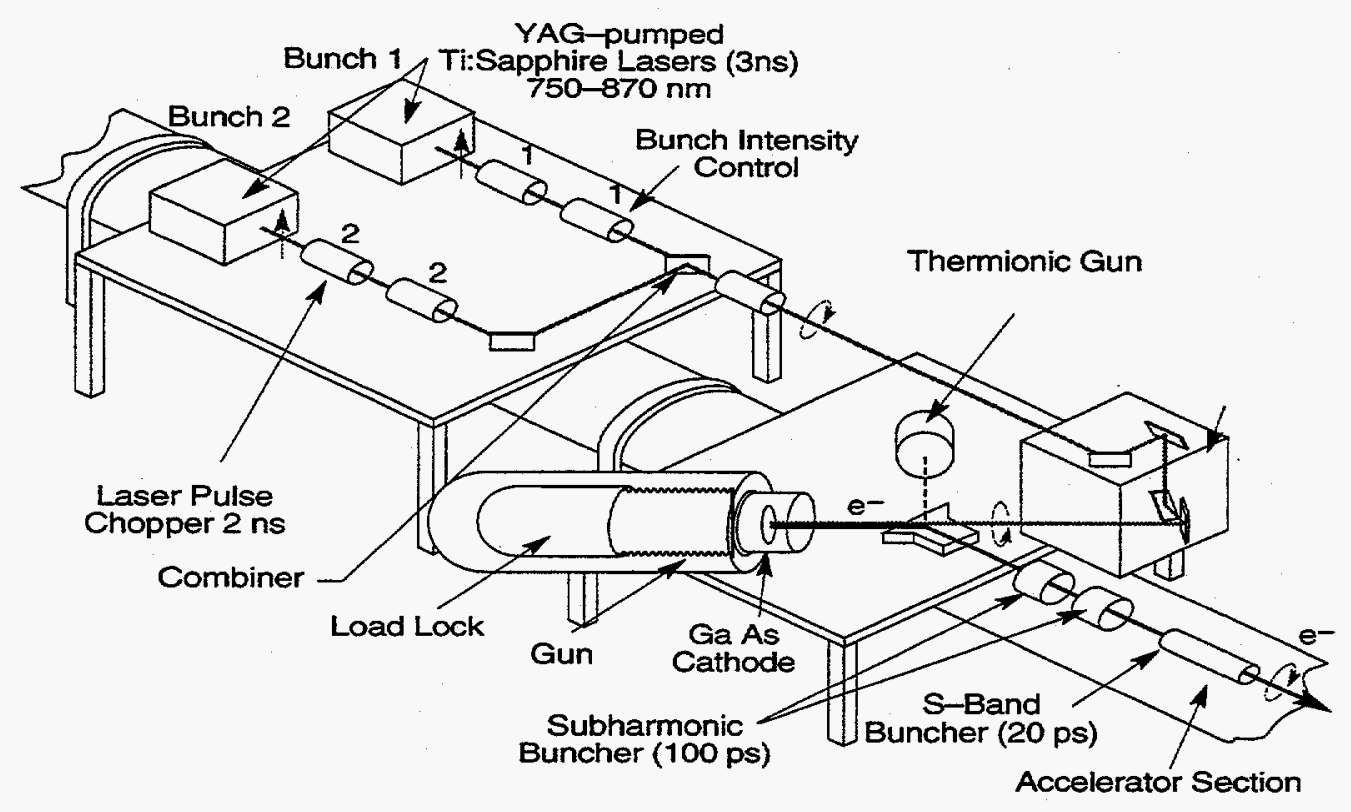

Figure 3.2: The electron source.

to $1.19 \mathrm{GeV}$ and sent into the electron damping ring to reduce emmitances. One bunch is accelerated down the LINAC to an energy of $30 \mathrm{GeV}$ and then is directed into the titanium positron target. Positrons resulting from the interaction are returned to the beginning of the LINAC where they are accelerated and sent into the positron damping ring. The remaining electron bunch as well as a positron bunch from a previous cycle of the accelerator are sent on to the end of the LINAC at which point they have been accelerated to an energy of $46.6 \mathrm{GeV}$. At the end of the LINAC dipole magnets guide the electrons into the north arc and the positrons into the south arc. About $1 \mathrm{GeV}$ of energy is lost in the arcs due to synchrotron radiation. After being focussed by a set of super-conducting quad triplets the bunches then pass through each other at the SLD interaction point where occasionally an interaction between a particle in the electron bunch and one from the positron bunch interact producing a region of $91.2 \mathrm{GeV}$ of energy. This then usually leads to the production of a $Z^{0}$ boson.

To achieve both reasonable luminosities and a stable interaction point the overlap of the beams must be small. The size of the beam-beam overlap at the interaction point is $\sim 2-3 \mu \mathrm{m}$ horizontally by $\sim 1-2 \mu \mathrm{m}$ vertically. The overlaps for LEP are 
considerably larger. It has an overlap of $\sim 250 \mu \mathrm{m}$ horizontally by $\sim 15 \mu \mathrm{m}$ vertically. The luminosity provided by SLC to SLD is

$$
L=\frac{f}{4 \pi} \frac{N^{+} N^{-}}{\sigma_{x} \sigma_{y}} H_{d} \eta
$$

where $N^{-(+)}$are the number of electrons(positrons) per bunch, $\sigma_{x, y}$ are the overlaps of the beams in the transverse dimensions, $f$ is the frequency of the collisions $(120 \mathrm{~Hz}), H_{d}$ is the enhancement factor due to beam-beam focusing, and $\eta$ is the machine efficiency[22]. With the performance parameters shown in Table 3.1, average luminosities of $(0.15,0.40,1.0) \times 10^{10}$ were achieved for runs occurring in 1992 , 1993 and 1994 respectively. The integrated luminosities were $0.3 p b^{-1}, 1.8 p b^{-1}$ and $3.6 \mathrm{pb}^{-1}$. The corresponding number of interactions producing a $Z^{0}$ is $10 \mathrm{k}, 50 \mathrm{k}$ and $100 \mathrm{k}$ where the relation is $N=\sigma_{e^{+} e^{-} \rightarrow Z^{0}} L$ and the the cross-section for producing a $Z^{0}$ is $30 n b$.

\begin{tabular}{|l|c|c|c|}
\hline & 1992 & 1993 & 1994 \\
\hline$\sigma_{x}$ & $2.4 \mu \mathrm{m}$ & $2.6 \mu \mathrm{m}$ & $2.5 \mu \mathrm{m}$ \\
$\sigma_{y}$ & $2.2 \mu \mathrm{m}$ & $0.8 \mu \mathrm{m}$ & $0.6 \mu \mathrm{m}$ \\
$N^{ \pm}$ & $2.8 \times 10^{10}$ & $2.9 \times 10^{10}$ & $3.5 \times 10^{10}$ \\
$\nu$ & $120 H z$ & $120 H z$ & $120 H z$ \\
$\eta$ & $60 \%$ & $70 \%$ & $65 \%$ \\
$H_{d}$ & 1.0 & 1.05 & 1.20 \\
\hline
\end{tabular}

Table 3.1: SLC operating performance.

\subsubsection{Beam Energy}

The beam energy was measured to be $91.2 \mathrm{GeV}$ with a spread of $60 \mathrm{MeV}$. The device used to make this measurement is called the energy spectrometer and has an accuracy of $0.020 \mathrm{GeV}$. There is no significant uncertainty in $R_{b}$ resulting from the $60 \mathrm{MeV}$ spread. This is due to the energy spread being small compared to the $Z^{0}$ width of $2.49 \mathrm{GeV}$ and the fact that it affects all the $Z^{0} \rightarrow q \bar{q}$ widths and largely cancels in the ratio. 


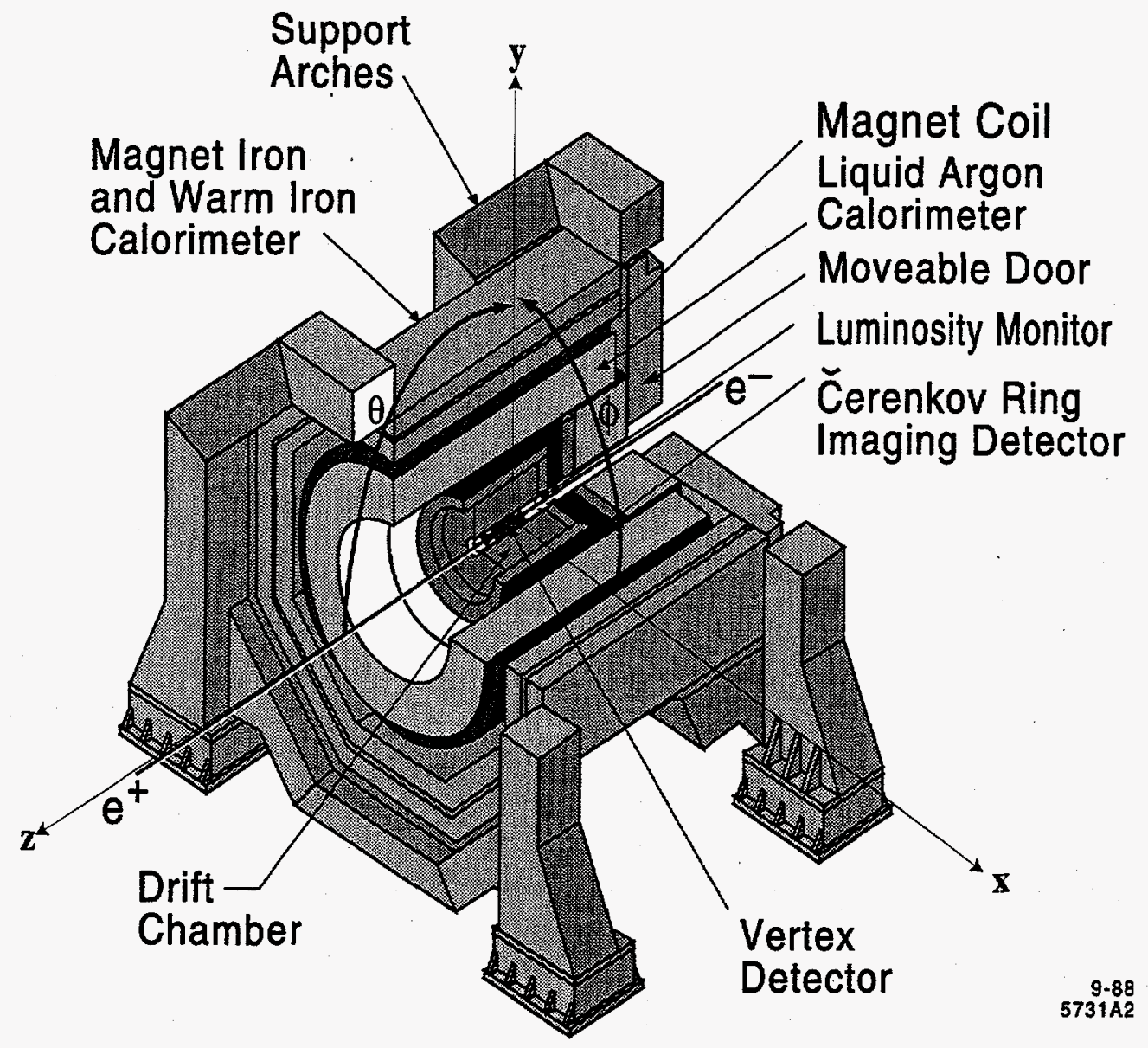

\subsection{The SLAC Large Detector (SLD)}

The SLD was commissioned in 1991 during an engineering run. This run was performed with all sub-detector elements except the full vertex detector. In 1992 the physics running commenced with the completed vertex detector installed and functioning. The detector now consists of the following sub-detectors in order of innermost to outermost[23]:

- The vertex detector is used for very high precision measurements of the trajectory of charged particles near the beam line (see Sect. 3.2.3).

- The drift chamber provides measurements of charged particle trajectories and through the interaction of the particles with the $0.6 \mathrm{~T}$ magnetic field it provides 
measurements of the particle momenta (see Sect. 3.2.2).

- The $\check{C}$ erenkov ring imaging detector is used for particle identification by measuring the velocities of particles from the radii of rings of $\check{C}$ erenkov radiation produced when a particle traverses it liquid and gas radiators at a velocity greater than the phase velocity of light in these materials. When combined with the momenta of the associated drift chamber track a particle mass is estimated. Using this, probabilities that the particle is an $e, \pi, K$ or $p$ are determined.

- A liquid argon calorimeter provides energy measurements for particles by sampling the energy of particle showers produced when they hit its dense high $\mathrm{Z}$ material (see Sect. 3.2.4).

- A warm iron calorimeter provides position and energy measurements of particles escaping the liquid argon calorimeter[24]

In the analyses of this thesis, only the tracking systems and liquid argon calorimeter are used. These devices and their role in the analyses are described in this section. First, we start with the SLD coordinate system which will be used in the detector descriptions and in the analysis sections.

\subsubsection{The SLD Coordinate System}

Frequently throughout this thesis the standard SLD coordinate system notation will be used. The coordinates are illustrated in Fig. 3.3. The $z$ coordinate is longitudinal to the beam-line and points along the positron beam direction. The $x$ and $y$ axes are transverse to the beam-line with $y$ in the direction opposite of gravity. The angle $\theta$ is measured off of the $z$ axis ( $\cos \theta=0$ corresponds to being completely in the $x y$ plane). Often, the $x y$ plane will also be referred to as the $r \phi$ plane. The dip angle is the angle measured to the $x y$ plane. Finally, $\phi$ is measured in the $x y$ plane counter-clockwise from the $x$ axis. 


\subsubsection{The Central Drift Chamber}

The passage of a charged particle through the tracking systems is identified by finding patterns of charge deposition in the Central Drift Chamber(CDC) consistent with belonging to a helical trajectory. The parameters describing the trajectories will be

\section{Radial Position of sub-detectors}

VXD :2.9cm to $4.2 \mathrm{~cm}$

CDC $: 20 \mathrm{~cm}$ to $100 \mathrm{~cm}$

CRID:1.0m to $1.8 \mathrm{~m}$

LAC $: 1.8 \mathrm{~m}$ to $2.9 \mathrm{~m}$

WIC $: 3.3 \mathrm{~m}$ to $4.5 \mathrm{~m}$
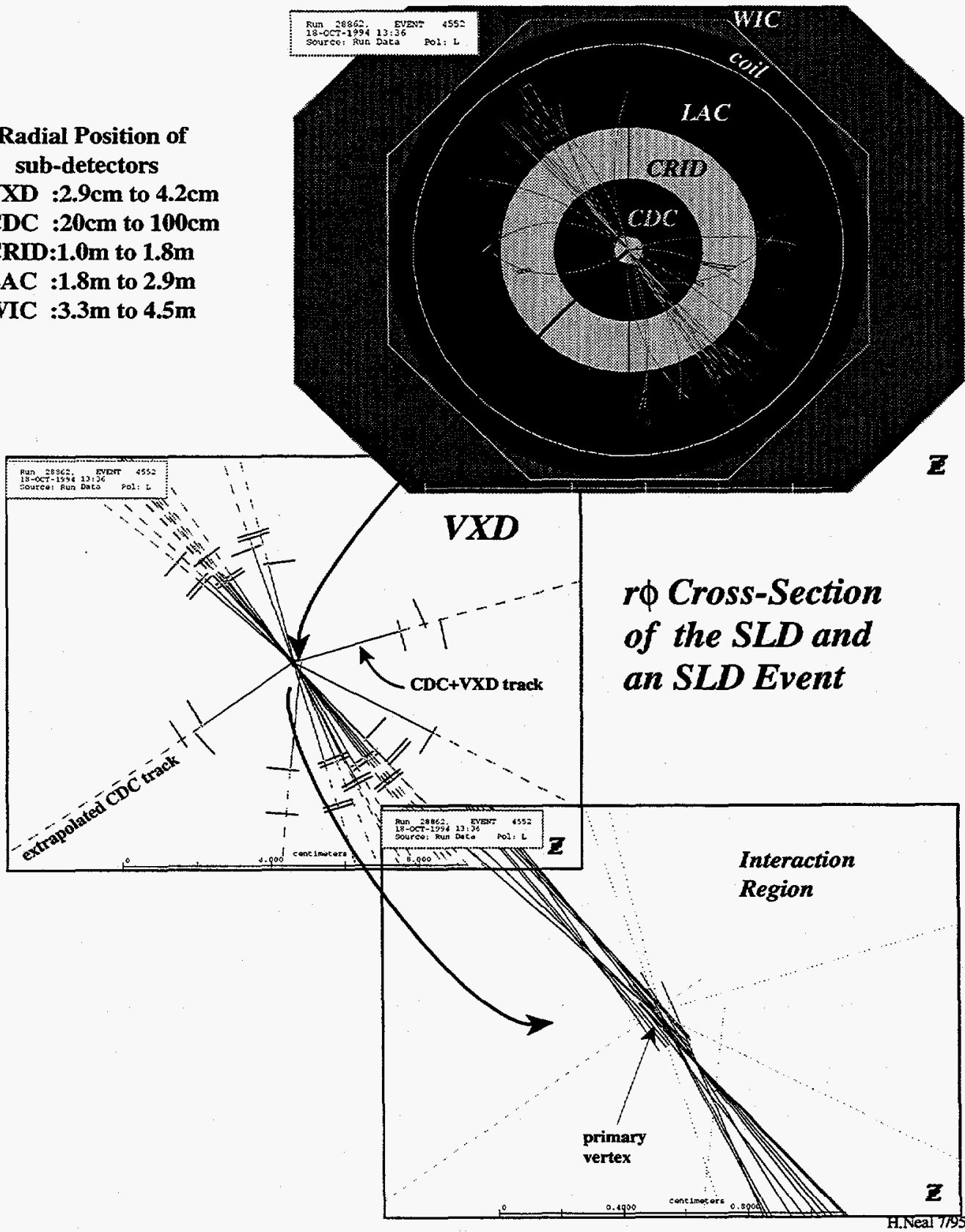


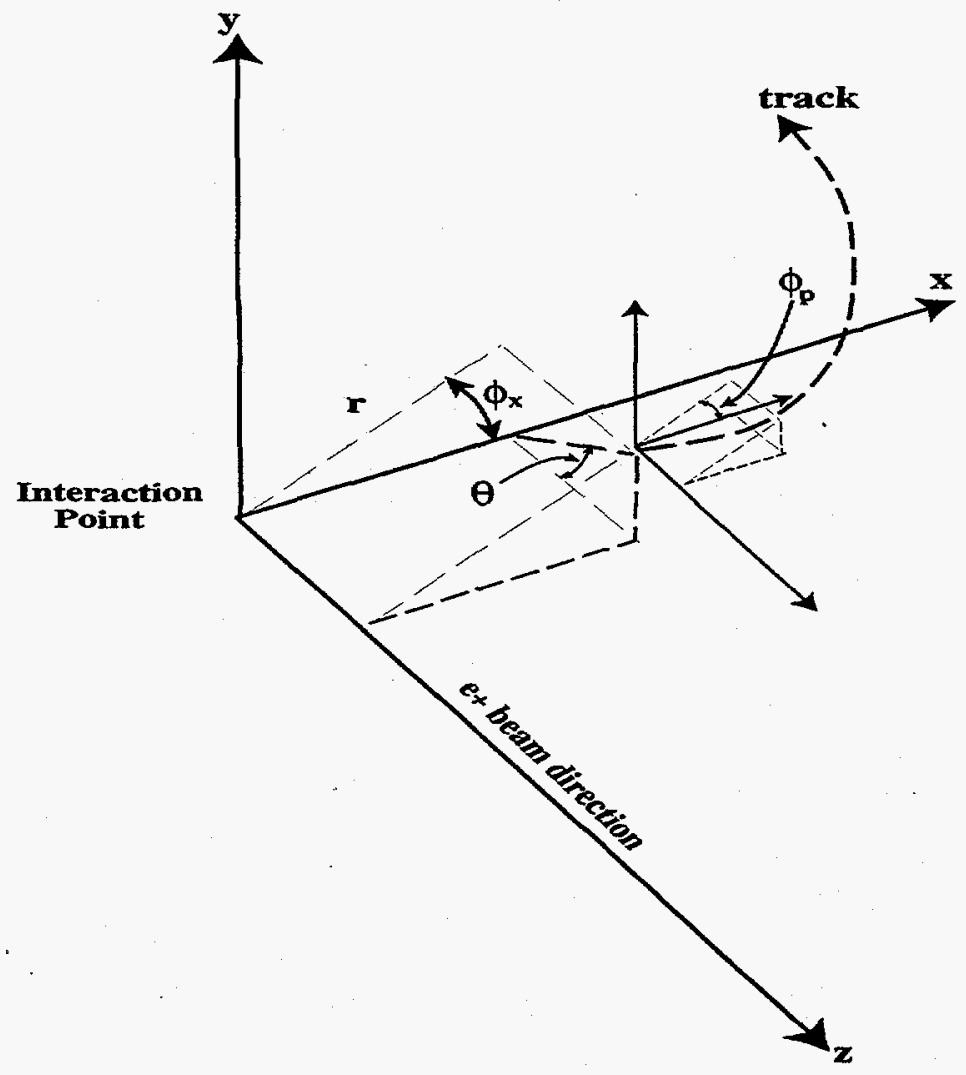

Figure 3.3: SLD coordinate definitions.

combined with hits in the vertex detector and the resulting parameters will be direct inputs in the variables used by the $b$-tags. The following aspect of the CDC make it useful for b-tagging:

- Good momentum resolution for accurately determining the direction that the $B$-hadron was moving before it decayed.

- High efficiency for identifying and reconstructing particle trajectories. (Any loss directly affects the efficiency for tagging events or hemispheres.)

- Good position measurement near the beam-line so that links to the proper vertex detector hits may be made for precise impact parameter measurements. 

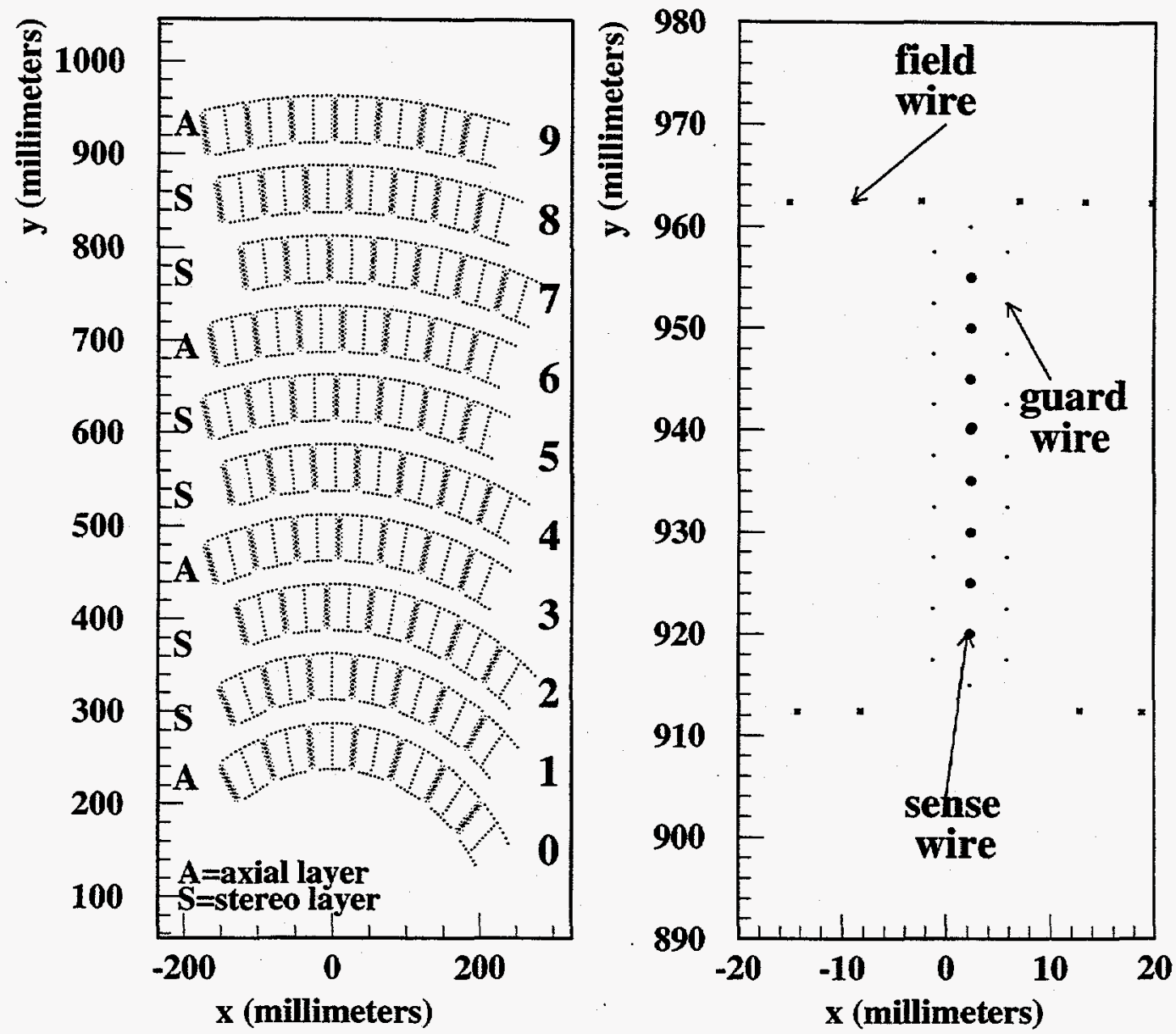

Figure 3.4: A section of the CDC endplate.

- Good dip angle resolution of the tracks. This is necessary as little assistance for this is provided by the vertex detector and it directly affects the $r z$ resolution of the track impact parameters.

These are achieved using a projective wire cell geometry which measures the position of ionization charges left by a charged particle traversing its gaseous volume. It is $\sim 180 \mathrm{~cm}$ longitudinally and extends from $20 \mathrm{~cm}$ to $100 \mathrm{~cm}$ radially relative to the beam-line. Tracks are identified out to $|\cos \theta|=0.85$. Inside the volume is a low drift 
velocity gas and wires parallel to the axis of the cylinder. The charge is collected on $25 \mu \mathrm{m}$ diameter gold plated tungsten sense wires. These are grouped into sets of eight which form a sense wire plane. At $4 \mathrm{~mm}$ from the sense wire plane on both sides are guard wires held at a voltage of $\sim 3 \mathrm{kV}$ and at $30 \mathrm{~mm}$ on either side are field wires held at a voltage of $\sim 5 \mathrm{kV}$. Several additional guard and field wires at the top and bottom of the cell complete the field shaping. These guard and field wires shape the potential and create the field necessary for amplifying the charge. A unit of sense and associated field wires is called a cell. Each superlayer consist of cells $50 \mathrm{~mm}$ along the radius and $\sim 59 \mathrm{~mm}$ wide in azimuth at the midpoint. The cells are grouped into concentric layers called super-layers. Each super-layer is one cell thick. There are four super-layers coaxial to the beam line, three with stereo angles relative to the beam line of $+41 \mathrm{mrad}$ and three with angles of $-41 \mathrm{mrad}$. Stereo layers of opposite angles are paired between axial layers. The relative position of the wires, cells, and layers are indicated in Fig. 3.4.

As a track passes through the $75 \% \mathrm{CO} 2-20 \% \mathrm{Ar}-5 \%$ Isobutane gas it causes an ionization charge to be left in the gas. The potential between the field shaping wires and the sense wires causes a cascade of charges which deposit on the nearest sense wire. The lines of constant potential and field strength are shown in Fig. 3.5. Also shown are the paths that the charge, caused by a track passing through the cell, would follow to the sense wires. A waveform representing the amount of charge deposited versus time is generated by the deposition of charge on the sense wire from each hit. This is read out on both ends along with the times of the arrival of the waveform after the beam crossing signal as given by SLC. The waveform information from each wire of the CDC is passed to the waveform sampling modules (WSM) contained in the fastbus system. From the waveform the charge deposition from the north $\left(Q_{n}\right)$ and south $\left(Q_{s}\right)$ sides of the wire are determined. Only hits which have charge deposition $\left(Q_{d e p}=Q_{n}+Q_{s}\right)$ greater than a threshold cut are used. The asymmetry of the north and south charge deposition is used to get a rough estimate for the longitudinal position of the hit as shown in Fig. 3.6. This information is used in the initial steps of recognizing a collection of hits as a track. More precise information from the arrival times of the hits and stereo information are used in the actual fit of the tracks. 

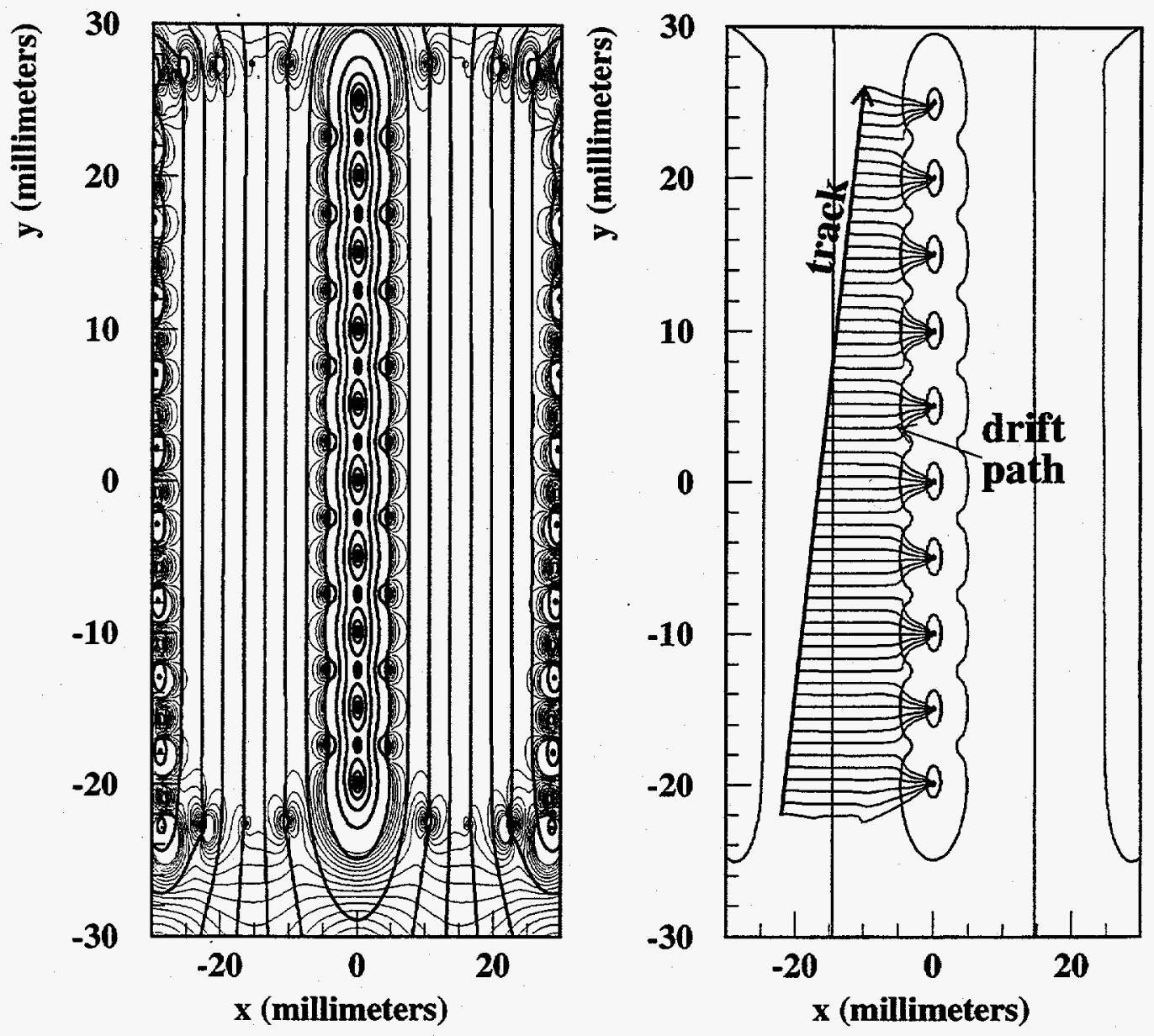

Figure 3.5: The left figure shows lines(bold) of constant potential and lines(thin) of constant field strength within a CDC cell. The right figure shows the drift path of charges caused by the passing of a track through the cell.

The recognition of a track in a large track multiplicity event can be a complex task. Unlike the brain which is capable of examining the entire event at once and then identifying patterns, the algorithms must work from the smallest elements(a hit) and then attempt to create the largest structure which fits a description of a track(a helix). The procedure used by SLD is to first identify segments of tracks called vector hits. These are a set of four or more hits within a cell of eight that are consistent with 


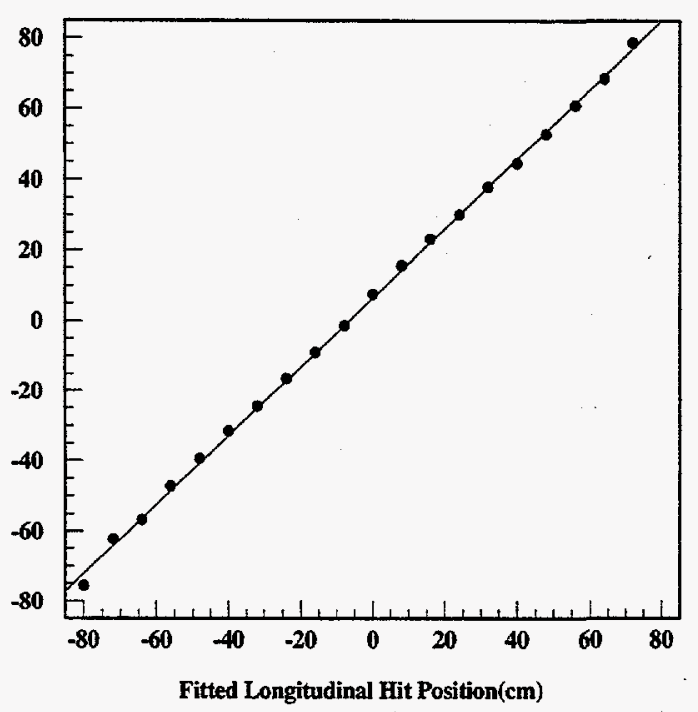

Figure 3.6: CDC hit $z$ position from charge asymmetry versus fitted $z$ position of hit.

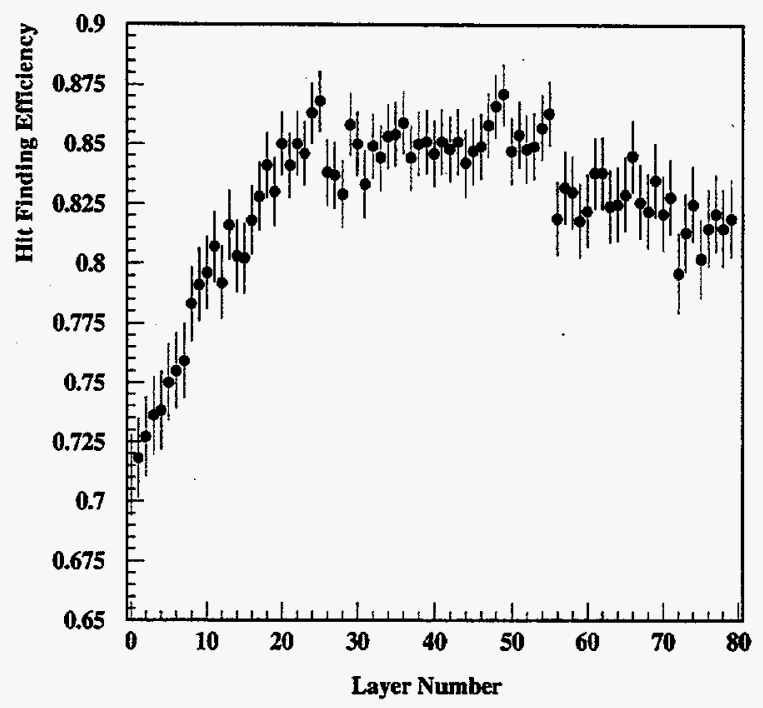

Figure 3.7: CDC hit finding efficiency versus layer number.

belonging to the same helix. Then, groups of vector hits on axial layers are compared for sets consistent with belonging to the same helix. The vector hits are added to the axial track segment depending on whether the angle of the stereo hit matches the track at the appropriate longitudinal position. The stereo angle causes stereo vector hits to rotate slightly as they are moved along the longitudinal axis. The $x$ and $y$ position dependence of the wires on the longitudinal position is given by Eqn. 3.1. Here $x_{0}, y_{0}$ are the position of the wires at $z=0, t_{x}, t_{y}$ are the direction cosines of the wires and $\theta_{\text {stereo }}= \pm 41 \mathrm{mrad}$.

$$
x=x_{0}+z / \cos \theta_{\text {stereo }} * t_{x}, \quad y=y_{0}+z / \cos \theta_{\text {stereo }} * t_{y}
$$

Once the tracks have been formed from the various axial and stereo hit combinations, they are ordered by length and quality and the best set of tracks not sharing vector hits is presented to the fitter. The efficiency for finding hits belonging to a track is shown in Fig. 3.7 versus the layer number where layer 0 is the innermost layer. The lower efficiency on the inner layers is a result of the higher backgrounds in those regions. The fitter makes an initial guess at the values of the parameters for the 


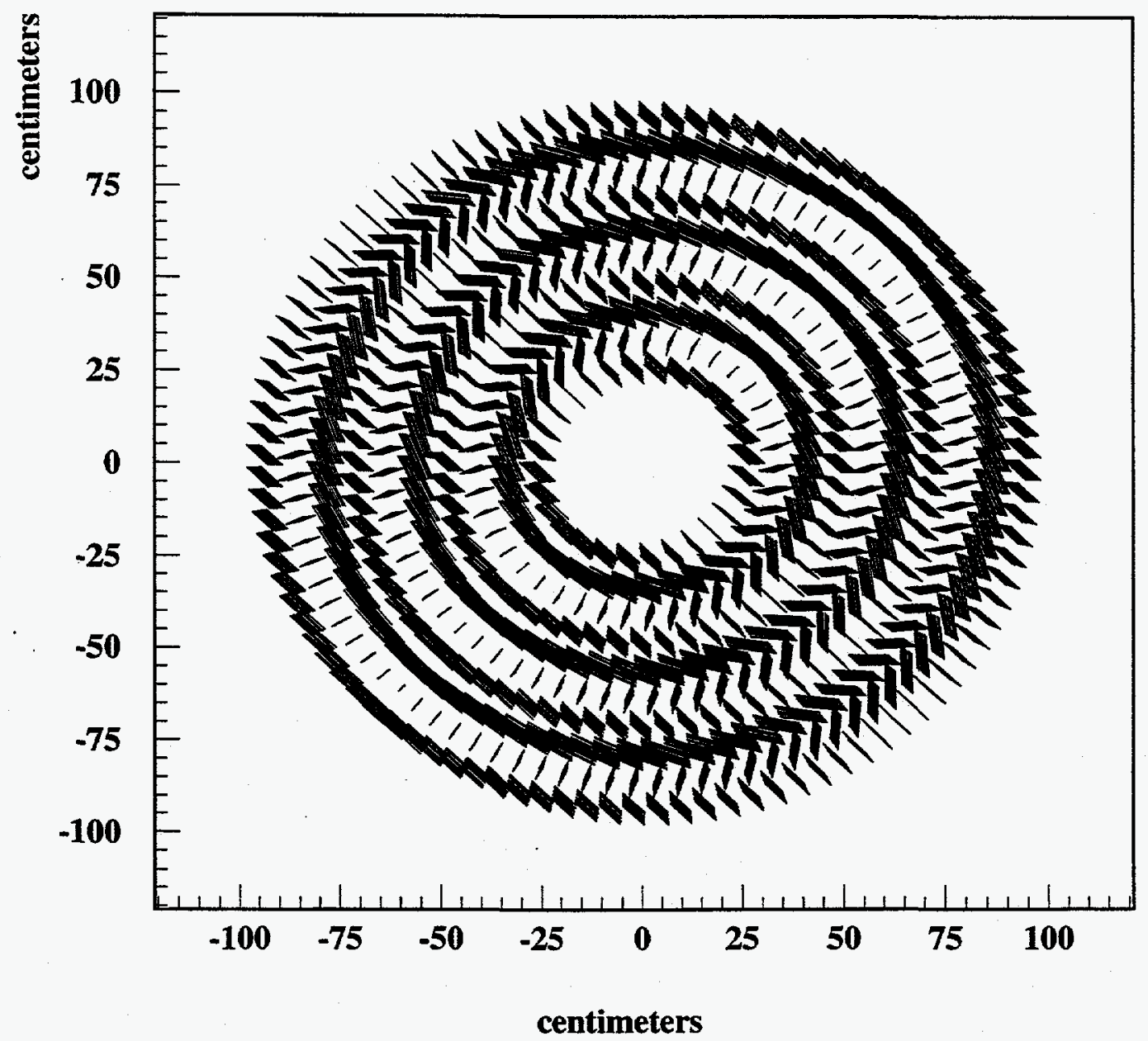

Figure 3.8: A slightly tilted view of the CDC sense wires showing the layout of the stereo wire planes. Only half of the wires are shown for clarity.

helix fit. Then by forming a weight matrix from the derivatives of the residual distances between the track hits and the helix with respect to the helix parameters and using these iteratively to minimize the $\chi^{2}$ of the fit the best set of helix parameters describing the track is obtained. 


\subsubsection{The SLD Vertex Detector}

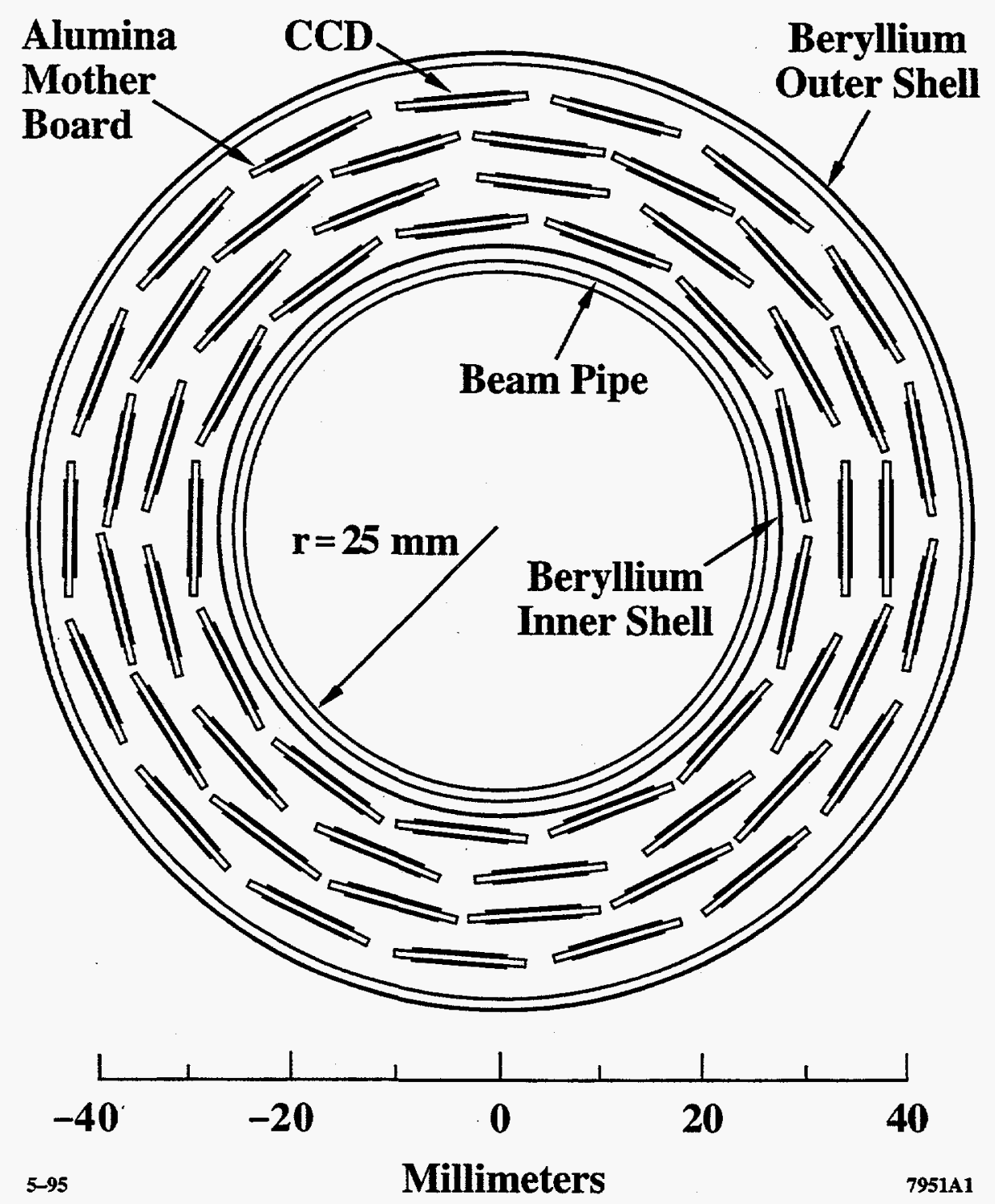

Figure 3.9: $x y$ cross-section of the VXD geometry.

The track hits from the high precision pixel vertex detector[26] reduce the uncertainty on the extrapolation of the track trajectories to the vertex decay points. It is the following aspects of this device which make it useful for the analyses:

- provides unambiguous precise track measurements near the interaction point 
- causes minimal scattering of the particles as they traverse the detector

The geometry of the VXD is illustrated in Fig. 3.9 and Fig. 3.10. It is a cylindrical device with an inner radius of $3.0 \mathrm{~cm}$ from the beam line and outer radius $4.2 \mathrm{~cm}$. The usable acceptance of the detector is $|\cos \theta|<0.71 \%$. It uniquely addresses the requirements listed above by using Charge Coupled Devices(CCDs). Almost all other vertex detectors use a silicon strip technology which have the disadvantage of measuring only the $r \phi$ position of the hits or using two separate planes to measure $r \not \phi$ and $z$ positions thus introducing more material and ambiguities in associating information from the two planes. The VXD is composed of $480 \mathrm{CCDs}$ which provide unambiguous 3-D information for each hit. The CCDs are metal-oxide-semiconductor(MOS) devices of $20 \mu m$ thick EPI silicon residing on $180 \mu m p^{+}$silicon substrate with a 2-dimensional array of 237,000 pixels. Each pixel is $20 \mu \mathrm{m}$ by $20 \mu \mathrm{m}[27]$. When a charged particle passes through a pixel, electrons are released from the epitaxial silicon and become trapped in the depletion layer of the pixel. The depth of the non-depleted silicon before the depletion region is $\sim 16 \mu \mathrm{m}$. The average energy needed to create an electron-hole pair in silicon is $3.62 \mathrm{eV}$ and the energy loss per unit length in silicon is $\frac{d E}{d x} \approx 3 \mathrm{MeV} / \mathrm{cm}$. Therefore, a charge of $\sim 80 e^{-} / \mu m[27]$ is generated by a minimum ionizing particle passing through the silicon. About half of the charge will diffuse into the depletion region resulting in a charge of $\sim 650 e^{-}$being deposited in the depletion region. The rows of charges are then clocked out to a special row on the edge of the chip called the $\mathrm{R}$ register. Charges in the $\mathrm{R}$ register are then output to the on chip FETs one at a time. It takes $90 \mathrm{~ms}$ to read out the entire detector. This means that several beam crossings of hits will be seen in an image but due to the high granularity of the detector this causes very little background. The average number of noise hits per CCD is 5. It is rare that a noise hit falls within the search area when combining VXD hits with the drift chamber track.

The CCDs are supported on a structure consisting of beryllium endplates and alumina boards(henceforth called ladders). There are 60 ladders each with 4 CCDs placed on each side of a $250 \mu \mathrm{m}$ thick ceramic board with printed tracks serving as electrical connections to CCD bond pads. The active detection region of the ladder is $9.2 \mathrm{~cm}$ longitudinally by $1 \mathrm{~cm}$ axially. The ladders are organized in four concentric 


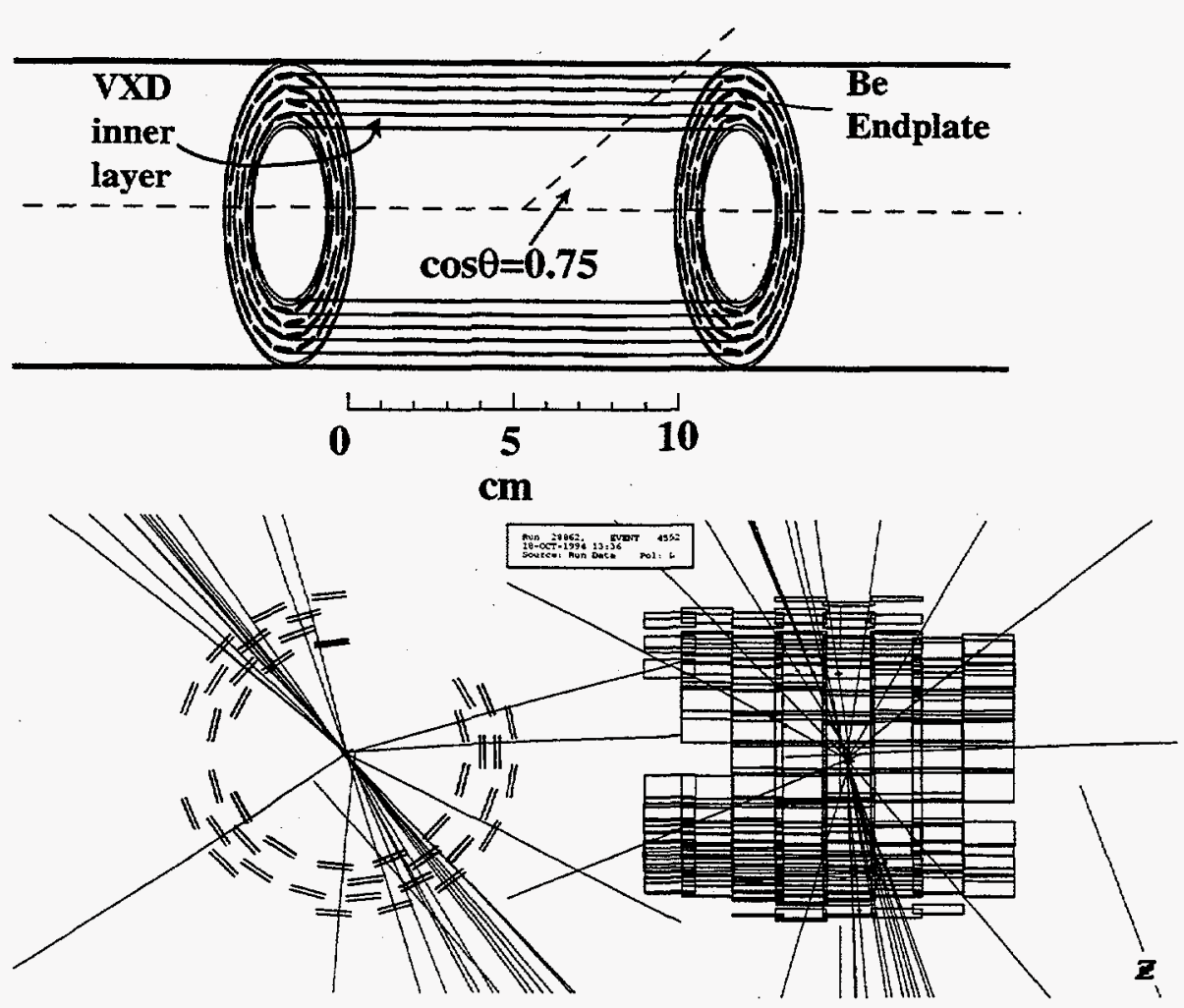

Figure 3.10: A $y z$ view of the VXD(top) and an event showing the VXD CCDs and pixel clusters on fitted tracks in the $x y$ view(bottom-left) and $r z$ view(bottom-right).

cylindrical layers, starting at $29 \mathrm{~mm}$ and extending to $41 \mathrm{~mm}$ from the beam line as shown in Fig 1. The VXD was originally designed for a $16 \mathrm{~mm}$ beam-pipe but due to backgrounds the inner radius had to be increased. This resulted in a much shorter lever arm than in the original design. A consequence is that while the $r \phi$ resolution is great, the dip angle resolution is not and the CDC must be relied on to assist in dip angle determinations. In a vertex detector upgrade to be made to SLD in 1995 this problem will be greatly ameliorated.

\begin{tabular}{|c|c|}
\hline Layer Number & Radius(cm) from Beam Line \\
\hline 1 & 2.9625 \\
2 & 3.3625 \\
3 & 3.7625 \\
4 & 4.1625 \\
\hline
\end{tabular}


The temperature of the VXD is maintained at $195 \mathrm{~K}$ to reduce the CCD dark current. It is cooled with cold $N_{2}$ gas flowing through a low mass cryostat surrounding the VXD. The ladder support system including endplates and barrel gas shells are made of beryllium to provide stability against thermal fluctuations. A $1 \mathrm{~mm}$ thick $\mathrm{Be}$ beam-pipe with a $100 \mu \mathrm{m}$ thick $T i$ liner are located at a radius of $25 \mathrm{~mm}$. Including the $0.5 \mathrm{~mm} \mathrm{Be}$ gas shell, the total material before the first CCD layer is $0.71 \%$ radiation length; each CCD-layer adds $\sim 1.1 \%$ radiation length but only about 2.3 CCDs are intersected by each track on average.

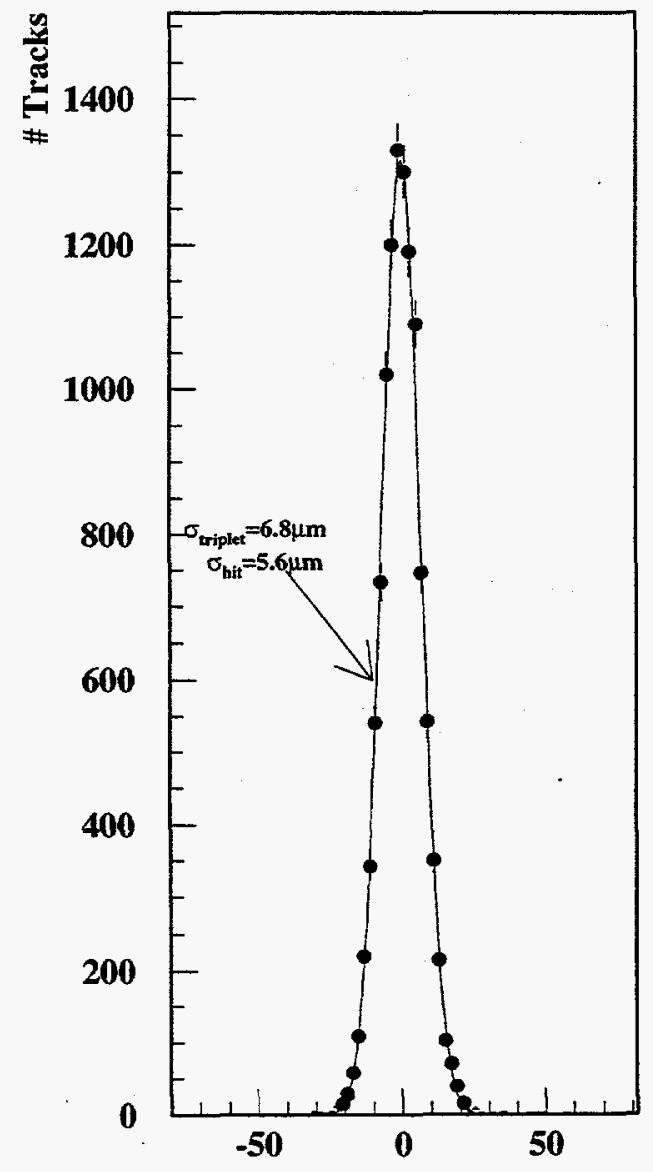

Triplet $\mathbf{r} \phi$ Residual $(\mu \mathrm{m})$

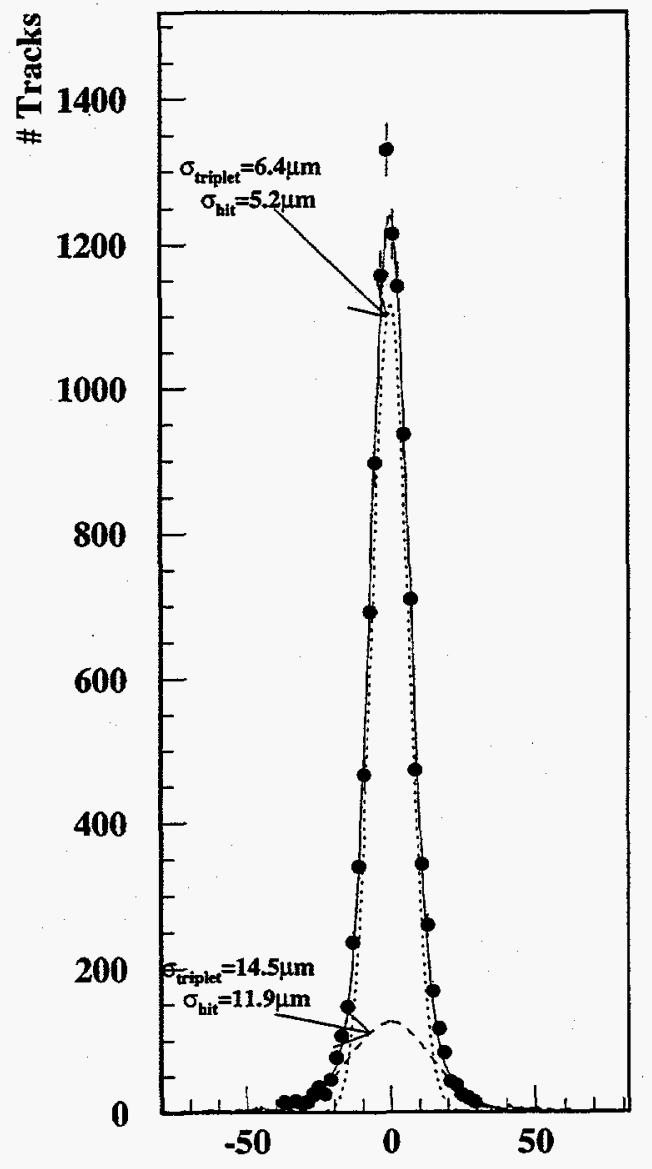

Triplet Z Residual $(\mu \mathrm{m})$

Figure 3.11: The VXD triplet miss distance. 
The hit resolution of the VXD is demonstrated in Fig. 3.11. Here, the residual distance between the trajectory going through two VXD hits separated by a layer and the hit on the middle layer is histogrammed in the $r \phi$ and $r z$ planes. Correcting for the resolution on the outer hits, the single hit resolution is determined to be $\sim 5 \mu \mathrm{m}$ in the $r \phi$ view and $\sim 5-12 \mu \mathrm{m}$ in the $r z$ view. The resolution in the $r z$ view increases as the dip angle increases.

Immediately after installation, $4 \%$ of the CCD channels were found to be dead due to inaccessible connection problems. No significant degradation attributable to radiation damage has been identified.

\subsubsection{The Liquid Argon Calorimeter}

The liquid argon calorimeter(LAC) is used to measure the energy of both charged and neutral particles. This information is provided versus position and allows the direction of the energy flow of the event to be determined[28]. This is then used to determine whether most of the charged tracks, on a given event, are likely to be contained within the tracking devices which have a lesser acceptance. The LAC consists of a central barrel section which covers the acceptance of the tracking systems and endcaps on either end completing the nearly hermetic coverage. The LAC barrel inner radius is $1.77 \mathrm{~m}$ and extends to $2.91 \mathrm{~m}$. The depth of the LAC is split into two layers for measuring the energy in electromagnetic showers and two layers for hadronic showers. In the barrel, the azimuthal and polar segmentations for the electromagnetic section are $\sim 33 \mathrm{mrad}$ and $\sim 66 \mathrm{mrad}$ in the hadronic section. The endcaps cover the region $8^{\circ}$ to $35^{\circ}$ from the beam line with similar segmentation. Altogether, the barrel and endcaps have a 21 radiation length thick electromagnetic section radially before a 2.8 absorption length hadronic section. The segmentation and thicknesses are chosen to maximize the amount of the particle energy sampled and to be able to differentiate between electromagnetic and hadronic particles. The energy resolution for electromagnetic showers in the LAC barrel is $\frac{15 \%}{\sqrt{E}}$ and $\frac{60 \%}{\sqrt{E}}$ for hadronic showers.

The main unit within the calorimeter is a module. An illustration of a typical set of electromagnetic and hadronic modules in the LAC barrel is shown in Fig. 3.13. A 


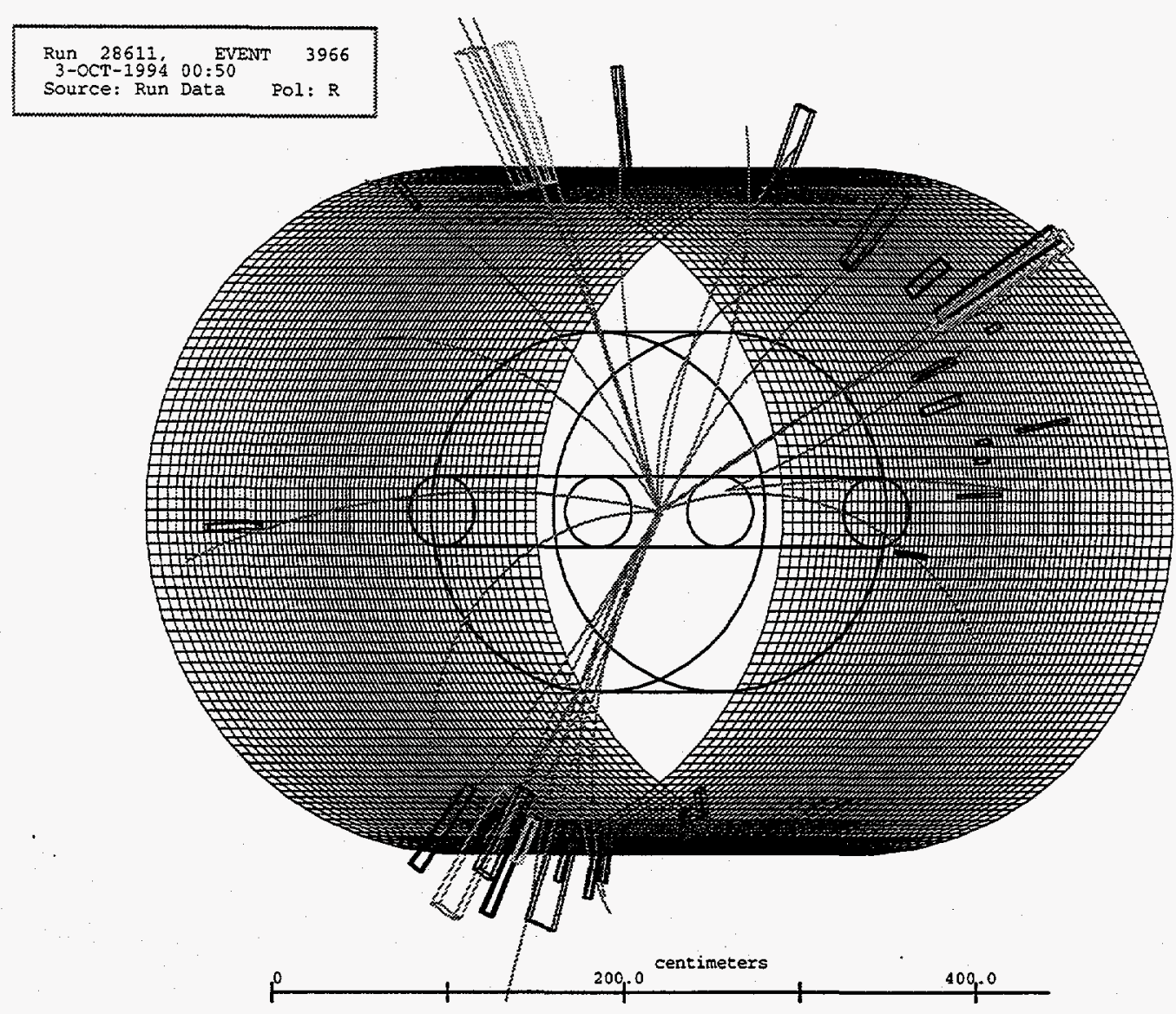

Figure 3.12: An SLD event showing tracks identified in the CDC and the associated energy deposits measured in the LAC.

module consists of stacks each with a lead plate held at ground which spans the full cross section, a gap of liquid argon, and then lead tiles held at a voltage of $2000 \mathrm{~V}$. As a particle passes through a module a shower of particles develops in the lead. The charges from the shower are pulled to the lead tiles through the argon by the potential between the tile and plate. Signals resulting from the charges on the plates are then processed, digitized and transferred to the data acquisition system. 


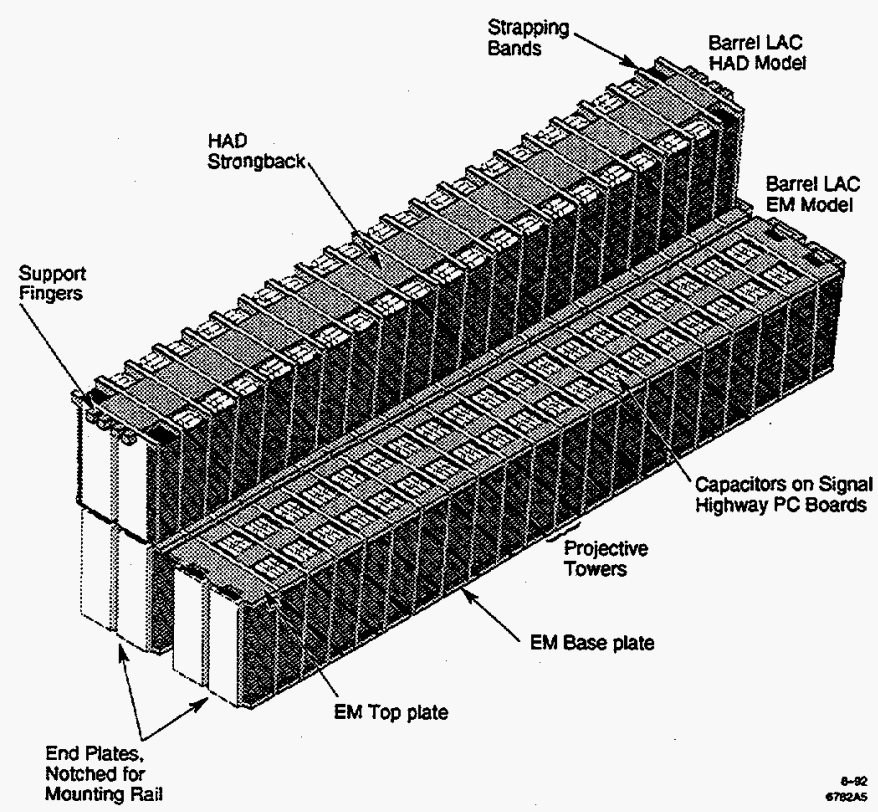

Figure 3.13: Electromagnetic and hadronic modules of the LAC barrel.

\subsubsection{Data Acquisition}

Signals from the detection systems are converted to serialized digital format and transferred over fiber optic cables to the acquisition hub called the fastbus system[29]. Then, the fastbus data processing modules apply corrections to the data including pedestal and gain corrections for the LAC data and snipping of waveforms from the CDC. The readout of the data is then controlled by an ALEPH Event Buffer(AEB) module for each system plus one for the trigger decision and readout of all subdetector systems. The trigger decision involves basic event characteristics from the subsystems. The trigger that is responsible for readout of the data used in the analyses of this thesis will be described in Sect. 7.1.1. If requirements for one of the triggers is satisfied, the data for the processed beam crossing is piped to the SLD VAX mainframe. The VAX transfers the data to tape and generates distributions of data characteristics that are used for quality monitoring. Detector status and beam condition indicators are logged with each event.

\subsubsection{SLD Runs and Run Number}


Data acquisition is done in units called runs with which a run number is assigned. Each unit corresponds to $\sim 4000$ triggers or $\sim 4$ hours. These units were chosen based on the period over which significant changes in the run condition are likely to occur and limits on processing power. The collection of runs between lengthy down times(months) is called an SLD run. It typically lasts 5-6 months with several breaks of up to a couple weeks for servicing of both the accelerator and the detector. The luminosity history for each SLD run is shown in Fig. 3.14.

For this thesis data from the 1992, 1993 and 1994 SLD runs were used. The event tag analysis uses data from the 1992 and 1993 run while the lifetime probability tag

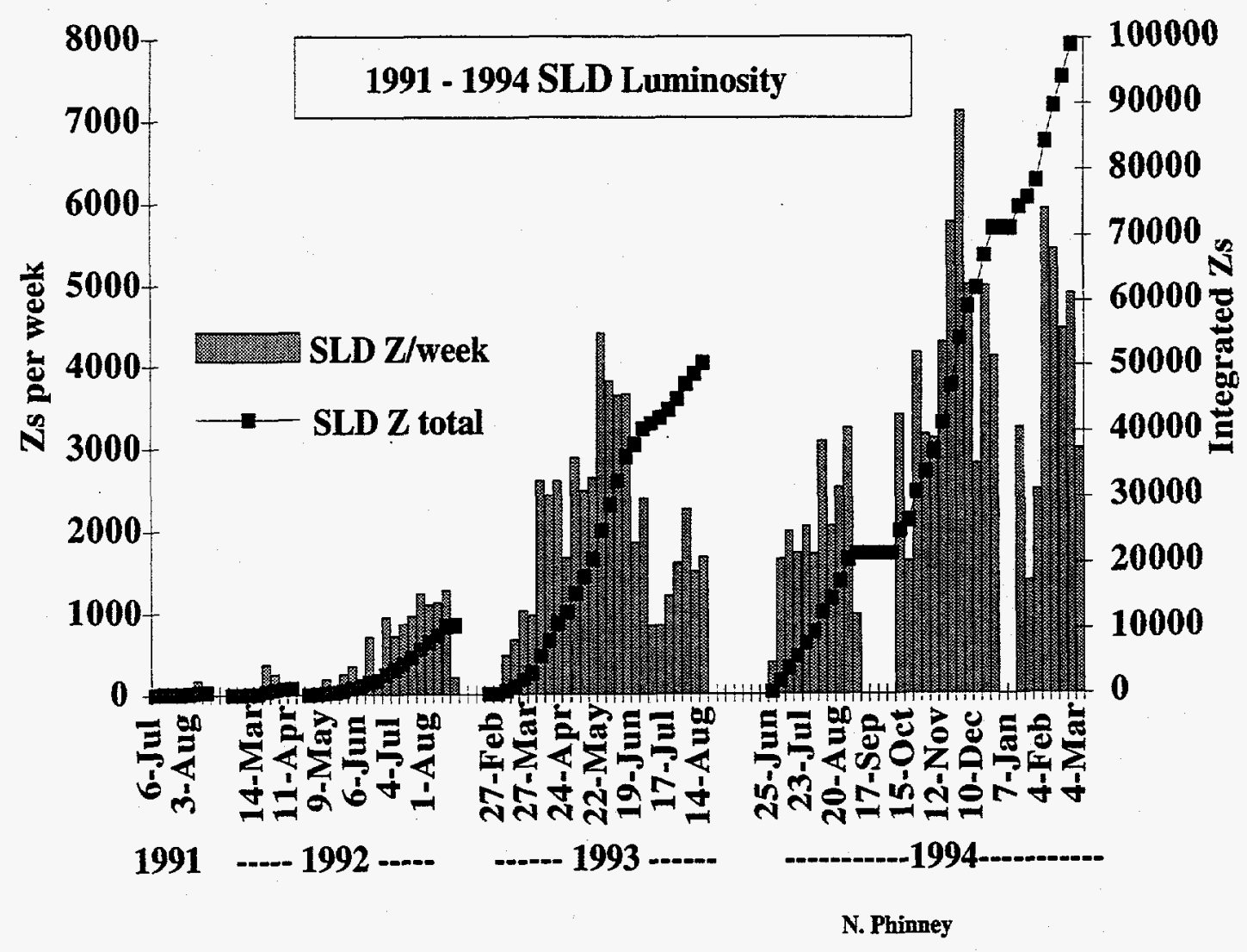

Figure 3.14: Luminosity versus time for all SLD runs. 


\begin{tabular}{|l|c|c|}
\hline SLD Run & Run Range & Number of $Z^{0}$ Events \\
\hline 1992 & $10000 \rightarrow 13700$ & $10 \mathrm{k}$ \\
1993 & $15772 \rightarrow 23700$ & $50 \mathrm{k}$ \\
1994 & $26400 \rightarrow 30700$ & $100 \mathrm{k}$ \\
\hline
\end{tabular}

Table 3.2: Run numbers corresponding to the three SLD runs.

analysis uses data from the 1993 and 1994 run. The distribution of the run numbers among the SLD runs is shown in Table 3.2.

\subsection{Detector Simulations}

The detector simulation is a model of the average response of the SLD to the passage of particles through its volume. The interactions with the detector primarily include scattering from the detector material and the signals that would be observed by the detection elements. The GEANT[31] version of the EGS simulator is used with the details of the detector material added. All pipes, electronics, gases, etc. have been detailed in the geometry. The material simulation is checked by whether it produces the expected resolution degradation and distribution of gamma conversions(see Appendix A). The output of the simulation is raw data, in the same format as from the real SLD, that models the detector's response to charged and neutral particles[23]. Improvements have been made to the simulation to include the effects of imperfect detector hardware and backgrounds coming from the accelerator. This is primarily accomplished by overlaying random triggers onto the generated $Z^{0}$ events. The random triggers are readouts of the detector taken approximately every 20 seconds on a beam crossing. Because of the low frequency for a beam crossing to produce a $Z^{0}$ the data from these triggers only indicate noise both from the detector and the accelerator beams and interactions of the beam with the beam-pipe. Any trigger whose data passes the filters for identifying $Z^{0}$ decays is not used. With the random trigger data is information about the status of the detector hardware. This includes the high voltage status and prevalence of detector readout errors. After the Monte Carlo simulated event and the random trigger data have been merged into the raw data 
format, the event is reconstructed with the same tracking and calorimeter clustering code.

\subsubsection{CDC Simulation}
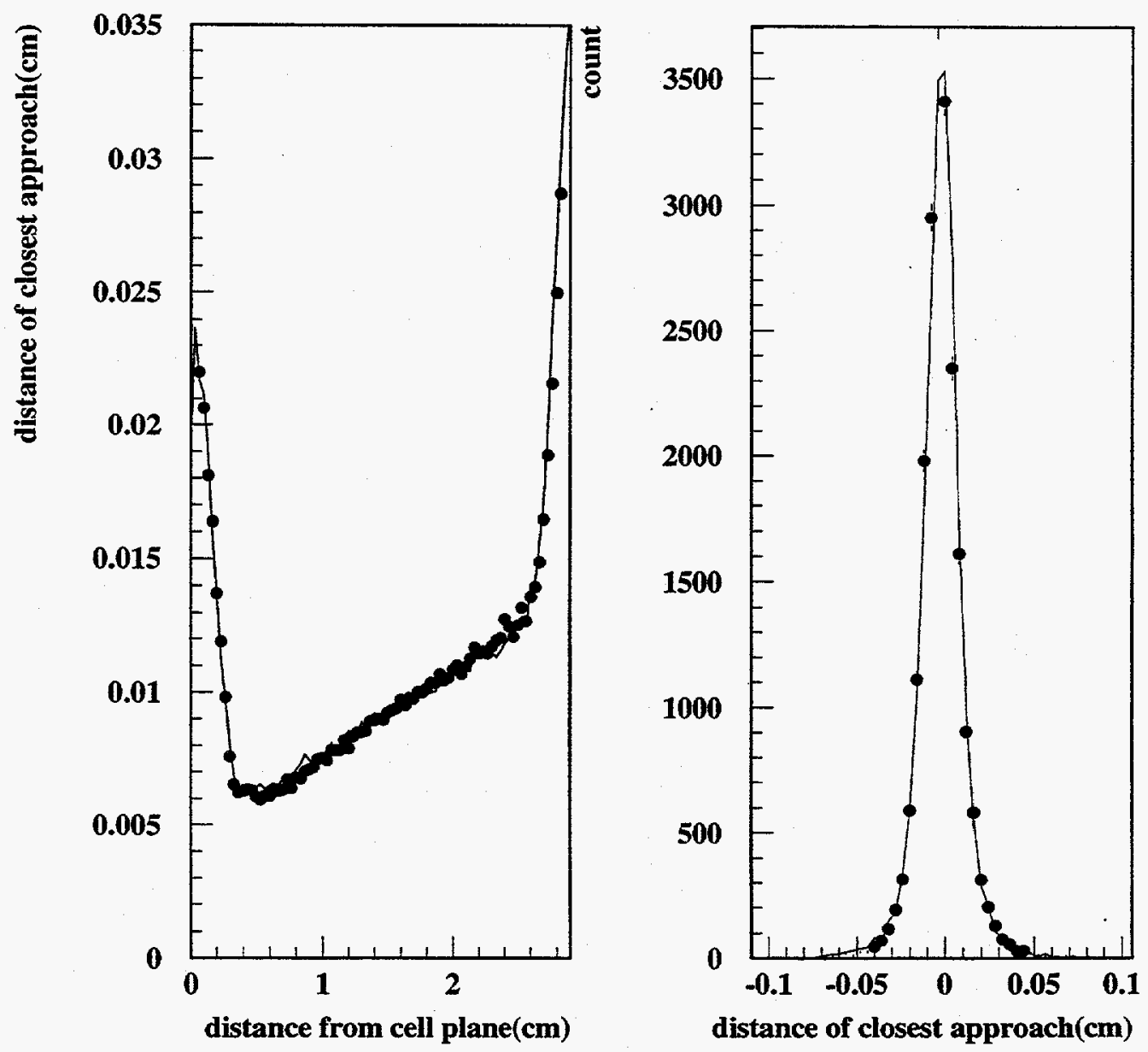

Figure 3.15: Plots of the distance of closest approach of the track hits to the helix describing the track. The left plot shows the increasing uncertainty in this distance as one moves away from the sense wire (or cell) plane. The uncertainty is $\sim 120 \mu \mathrm{m}$ on average for tracks with momentum $>5 \mathrm{GeV} / c$.

The nominal CDC simulation handles detector scattering from detector material and a simplified model of charge deposition and pulse heights. The simulation has been 
modified to better simulate the real efficiency of the chamber. Misalignment effects, hardware status, hit resolution, error records, hit time smearing, and hit loss near wire ends have been added. The modifications are implemented as follows:

\section{- CDC Internal Alignment}

To simulate the uncertainty in the alignment of the CDC cells, ten different maps of the cell geometry with distributions of the displacements consistent with the alignment errors were used. In the procedure for generating the maps any overall rotational offsets of superlayers were removed. The map is selected at the simulation stage based on the event number. Later, at the reconstruction stage, the ideal cell geometry is used. The drift model errors are modified to incorporate the alignment uncertainty. Thus, the resulting $\chi^{2}$ still has the proper peak position and width.

\section{- Two Hit resolution}

The time with which two hits received on the same wire may be resolved with rises from $0 \%$ efficiency at $80 \mathrm{~ns}$ to a plateau at full efficiency over a period of $\sim 25 \mathrm{~ns}$. The probability for resolving the hits is modeled as

$$
\text { probability }=1-e^{-(\Delta t-80 n s) / 25 n s}
$$

Hits with time separation $\Delta t$ are eliminated 1-probability of the time.

\section{- High Voltage Failures}

When a large charge builds up in the gas from high backgrounds from the accelerator the current required to maintain the potential between the field and sense wires can increase enormously. The potential for damaging wires due to high current spikes exists. The chamber is protected by automatically turning off the high voltage to a layer of the chamber when the current exceeds a cut. Usually a set of eight layers(a super-layer) will be shutoff instead of individual layers. This leads to large segments of all tracks in the events occurring during such a period to be missing. This must be simulated as these situations lead to increased uncertainty in the position measurements. Of all hadronic events 
collected approximately $10 \%$ occurred while a layer or layers of the CDC were tripped. The background events include the high voltage levels of the superlayers and if the high voltage is below $95 \%$ of normal the hits to these layers from the Monte Carlo are removed.

\section{- Error Records}

Error records occur when the information from the waveform can not be deciphered. This can result either from machine backgrounds or sporadic failures of the electronics. When an error record occurs only information about the period of the disturbance is returned. The start time of the error is randomly selected. Hits found to fall within an error record are eliminated.

- Hit Loss near Wire Ends

At the wire ends are the grounded endplates for the chamber. These distort the drift field and cause significant inefficiencies within $5 \mathrm{~cm}$ of the wire ends. This has been parameterized both in the distance from the wire end and the dip angle of the track. This is then used to remove Monte Carlo hits to produce the same distribution as observed in data.

\subsubsection{VXD Simulation}

The background hits plus uncertainty in the CCD positions, ladder to ladder alignment, radial shape of the ladders, and orientation with respect to the CDC are simulated. Backgrounds are mainly the result of the long readout time of the events $(90 \mathrm{~ms})$ which is approximately 16 beam crossings. They also come from SLC backgrounds where muons occasionally travel nearly parallel to the surface of the CCD and a track segment along the CCD is observed. The uncertainty in the position of the detector elements is simulated by smearing the positions within the measured uncertainties relative to a nominal geometry on each event. 


\subsubsection{LAC Simulation}

Online calibrations of the readout electronics are performed and stored with the raw data. However, this procedure can not identify shorted/dead towers and not all noisy towers. The raw data is divided into periods of $\sim 1 d a y$ and a search for channels with hits high compared to other channels on the same layer is performed. Also, checks on acquisition periods spanning $\sim 1$ week are searched for channels with no signal. Very noisy channels and channels that appear to be dead in the data are eliminated from the Monte Carlo for the corresponding period.

\subsection{Detector Performance Checks}

The performance of the tracking systems is checked using reconstructed $\mu^{+} \mu^{-}$events and by comparing tracking distributions from the data to the Monte Carlo simulation distributions. The results of these checks are described below.

\subsubsection{Checks using $Z^{0} \rightarrow \mu^{+} \mu^{-}$Events}

A set of $Z^{0} \rightarrow \mu^{+} \mu^{-}$events from the 1993 and 1994 runs have been used to study the tracking performance. Such events are useful because their momenta are well known, they suffer little from interactions with material, they are produced back to back and they come directly from the primary vertex. The $\mu^{+} \mu^{-}$tracks are required to be within $|\cos \theta|<0.6$. In addition, loose cuts have been applied to purify the sample. These include selecting events with two tracks of opposite charge, and requiring that both tracks have $r \phi$ impact parameters less than $2 \mathrm{~mm}, z$ relative to the measured primary vertex position at the $r \phi$ impact point less than $1 \mathrm{~cm}$ and transverse momentum greater than $10 \mathrm{GeV} / c$. Using these, the following distributions have been studied.

\subsubsection{Dip Angle Resolution}

The accuracy of the dip angle $(\lambda)$ measurement of a track is determined by summing the $\lambda$ s for the track from each $\mu$. Ideally, the value would always be zero since the $\lambda$ of 


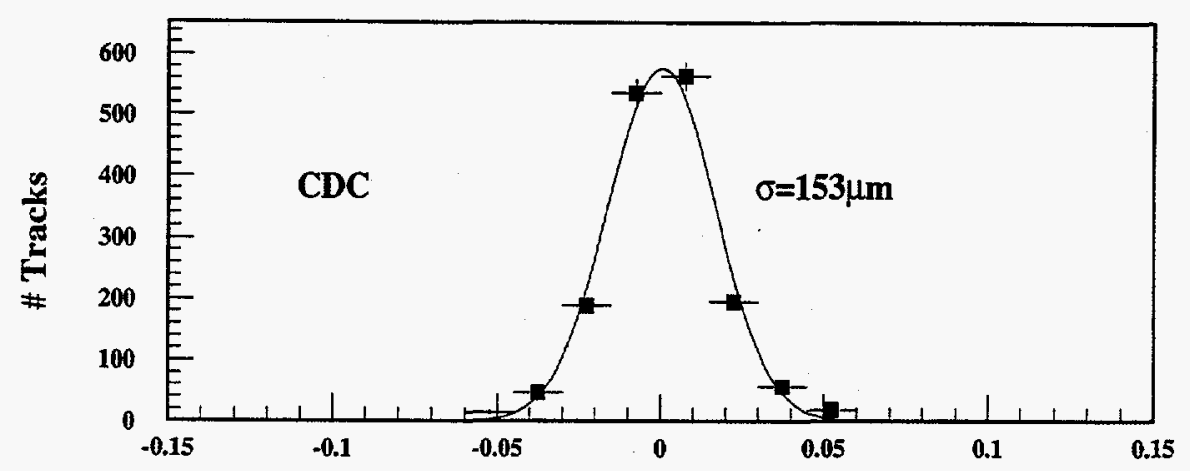

$x y$ impact parameters(cm) with respect to the $P V$ in $\mu^{+} \mu^{-}$events

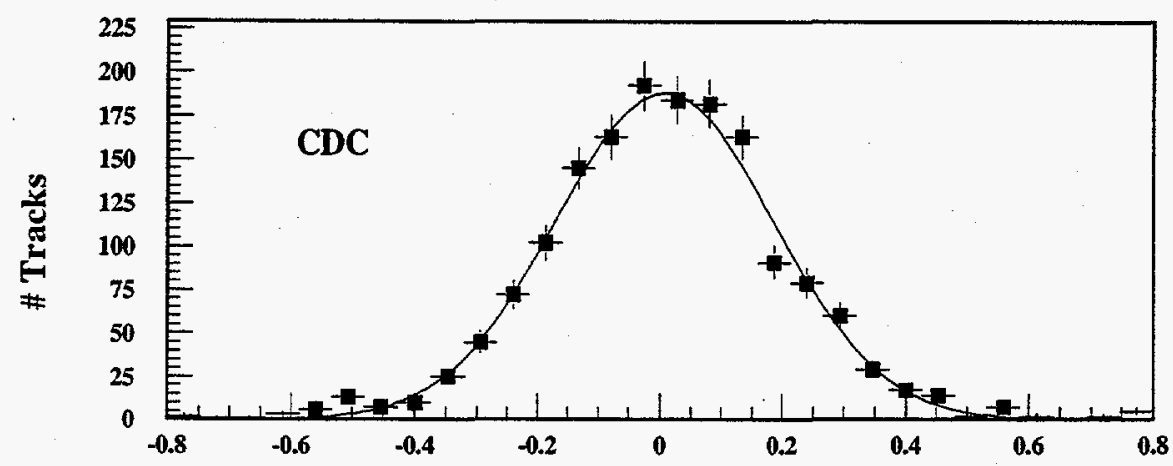

3-D impact parameters(cm) with respect to the $\mathrm{PV}$ in $\mu^{+} \mu^{-}$events

Figure 3.16: $r \phi$ and 3-D impact parameters for CDC alone tracks from $\mu^{+} \mu^{-}$events.

one muon is the opposite sign and same magnitude as the other ( $\lambda$ is negative for $-z$ ). The distribution of the $\lambda$ sum is shown in Fig. 3.17 and has a width of 6 mrad. Since this represents the combined uncertainty on the $\lambda$ measurement of both tracks, the $\lambda$ resolution for a track is $\sim 4 \mathrm{mrad}$. The resolution for a track improves to $2.5 \mathrm{mrad}$ for CDC+VXD tracks. This is shown in Fig. 3.18. Due to multiple scattering and smearing of waveforms as a track becomes more parallel to the CDC sense wires, the resolution degrades with increasing angle. 


\subsubsection{Inverse Momentum}

The inverse momentum $\left(p^{-1}\right)$ distribution indicates how well the curvatures of the tracks are being measured. The distributions are shown in Fig. 3.17 for negative and positive charge tracks. The widths of the distributions for $p^{-1}$ is $0.005 \mathrm{GeV}^{-1}$ for both $\mu^{+}$and $\mu^{-}$tracks. Differences in the widths of the distributions would indicate internal detector alignment problems. The $p^{-1}$ resolution is $0.0035 \mathrm{GeV}^{-1}$ for tracks from $\mu^{ \pm}$when CDC+VXD tracks are used. This is show in Fig. 3.19. It represents a $\sim 30 \%$ improvement over the resolutions obtained using only the CDC.

\subsubsection{Impact Parameters}

The CDC impact parameter distributions are shown in Fig. 3.16. In the $r \phi$ plane a resolution of $153 \mu \mathrm{m}$ is observed. A negligible contribution to the resolution comes from the primary vertex position uncertainty. The CDC 3-D impact parameter distribution is actually composed of two distributions due to phase space. This becomes clearer with the higher resolution obtained with VXD hits added in the track fit. While the impact parameter resolutions achieved with the CDC alone are not sufficient for tagging tracks as coming from $B$-decays, they are sufficient to be used in conjunction with the vertex detector hits to achieve the required resolution. Fig. 3.18 shows the impact parameter distributions obtained when the VXD hits are combined with the CDC track. Approximately a factor of ten improvement in the impact parameter resolutions is observed.

\subsubsection{Tracking Performance vs. Monte Carlo Prediction}

The Monte Carlo simulation represents our knowledge of how the SLD responds to particles traversing the detector and therefore predicts how well the particle trajectories will be reconstructed. Variables which are sensitive to the track reconstruction quality are compared between data and Monte Carlo to identify problems. Poorly matching distributions can indicate problems with the gas mixture of the CDC, hardware electronics problems (e.g. the loss of a VXD ladder), radiation damage, or simply poor calibration constants such as incorrect drift velocity corrections used for the 


\section{CDC Tracks in $\mu^{+} \mu^{-}$Events}

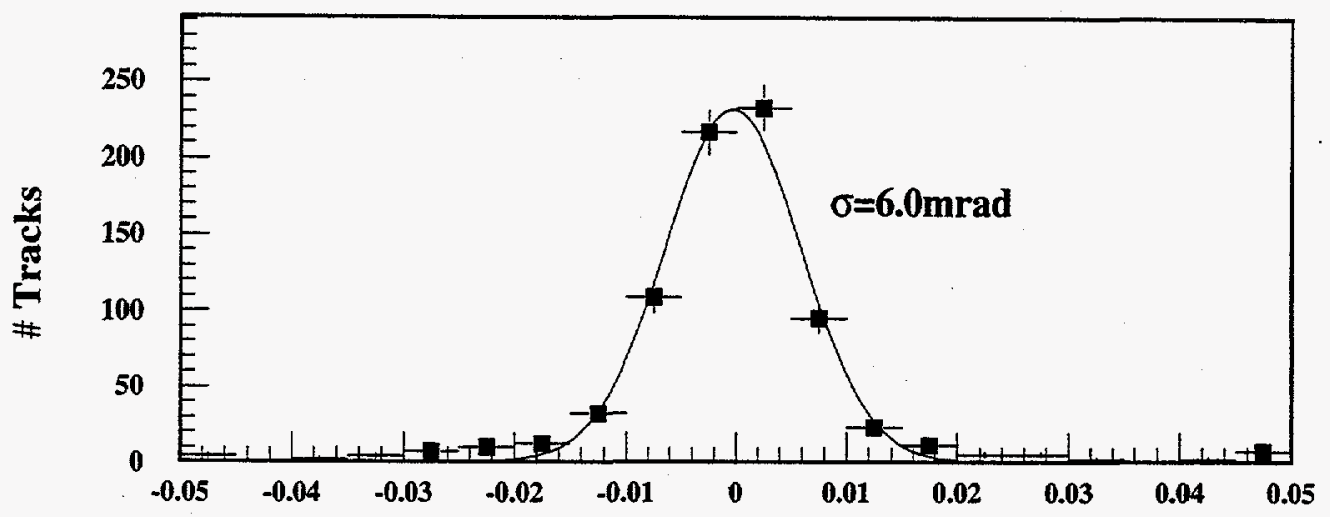

Dip Angle Sum
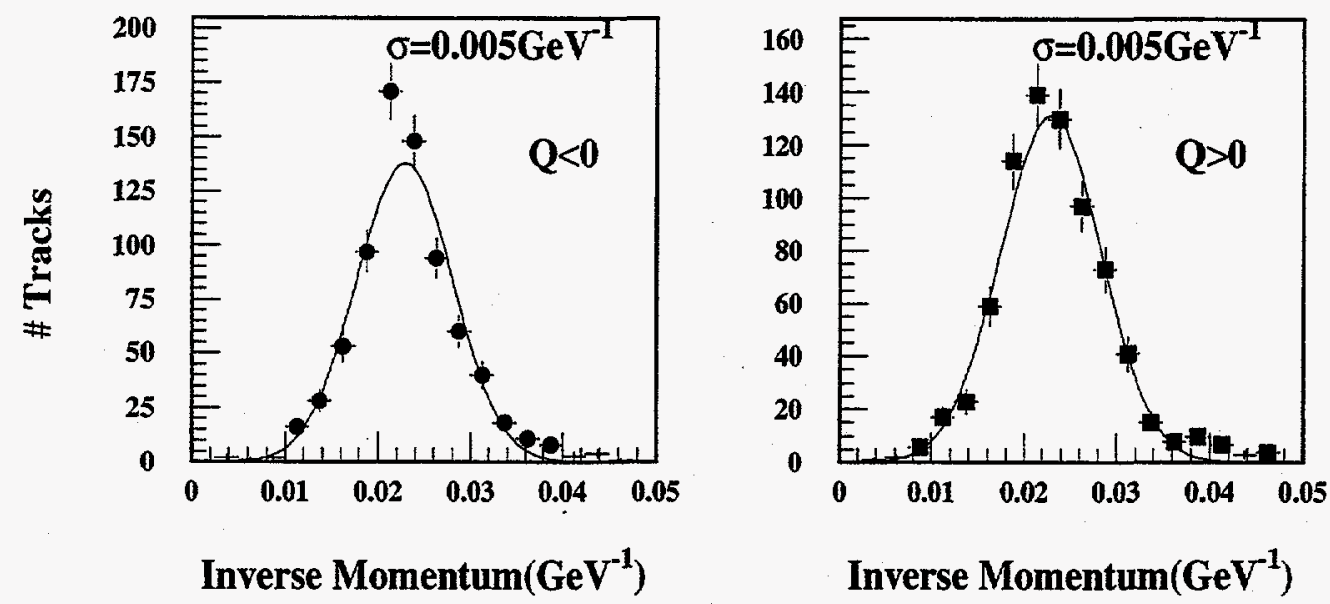

Figure 3.17: Dip angle resolution and momentum resolution from CDC alone tracks in $\mu^{+} \mu^{-}$events.

CDC track reconstruction. As monitors of the tracking performance the following quantities are used: the fit quality of tracks, the number of hits on the tracks, the impact parameters of the CDC track, the miss distance for $\mu$-pairs, the number of vertex hits per track and the distributions of the number of good tracks and fraction of those good tracks that link as a function of position. An event selection has been 


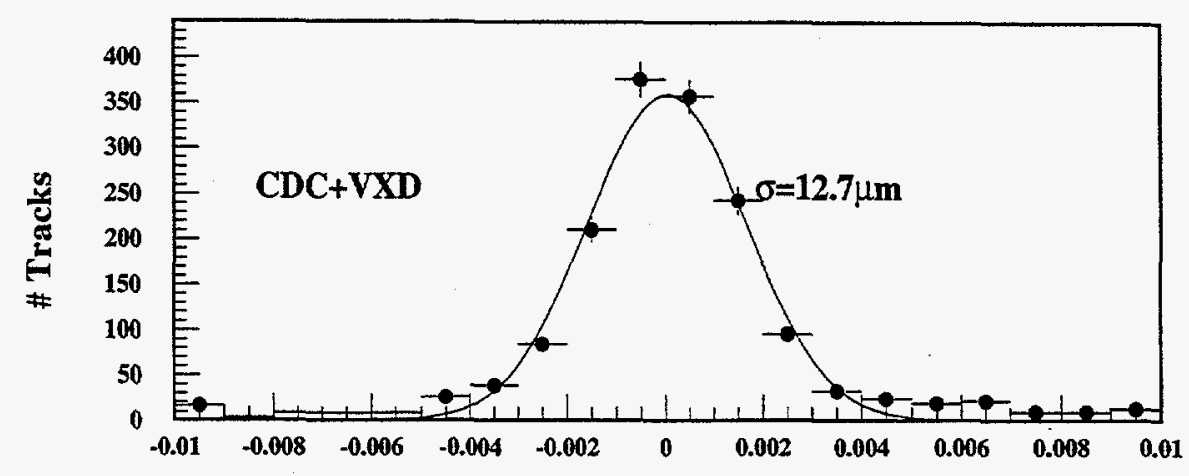

xy impact parameters $(\mathrm{cm})$ with respect to the $\mathrm{PV}$ in $\mu^{+} \mu^{\circ}$ events

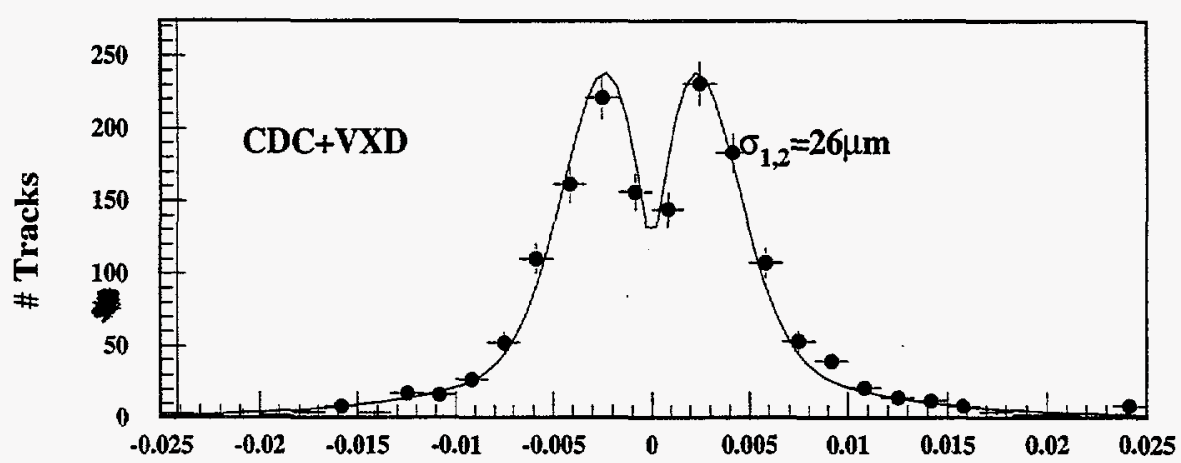

3-D impact parameters(cm) with respect to the PV in $\mu^{+} \mu^{-}$events

Figure 3.18: $r \phi$ and 3-D impact parameters for CDC+VXD tracks from $\mu^{+} \mu^{-}$events.

applied to both Monte Carlo and data to insure that only quantities from hadronic events contained within the acceptance of the tracking systems are being compared. Loose cuts have been applied to the tracks to select only good tracks from the $e^{+} e^{-}$ interaction.

The $\chi^{2}$ distribution for the CDC tracks indicates whether the drift model is correctly predicting the uncertainties on the drift distances measured for each of the hits. If it is, the distribution should peak at 1.0 and have a width of 1.0. Improper distributions would also indicate potential alignment problems or bad drift constants. As shown in the data and Monte Carlo distributions of Fig. 3.20 no such problems are evident. 
CDC+VXD Tracks in $\mu^{+} \mu^{\cdot}$ Events

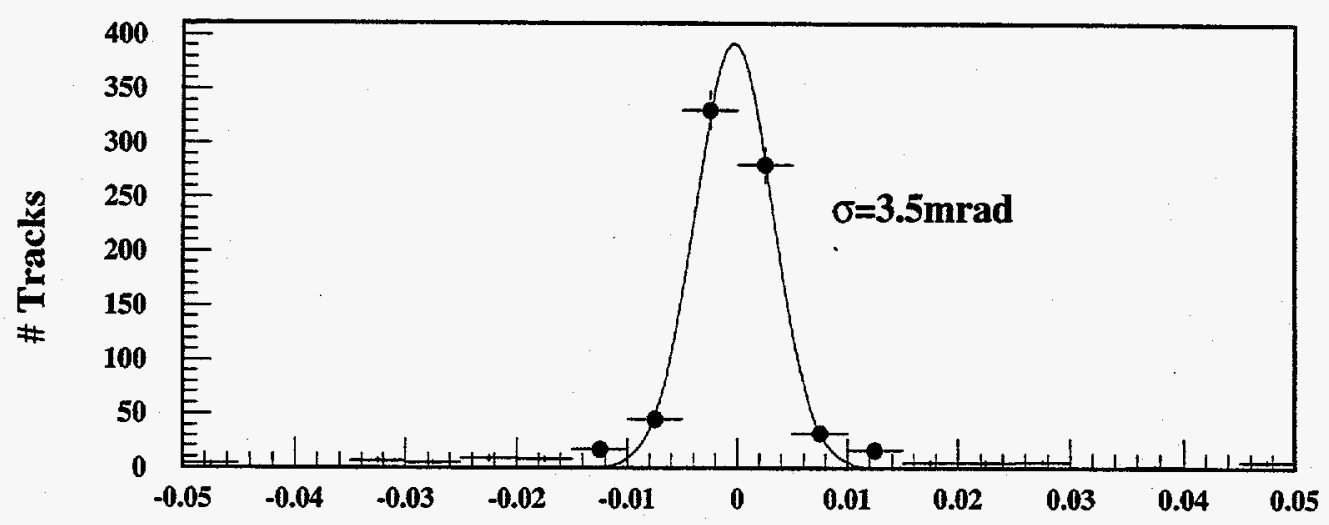

Dip Angle Sum

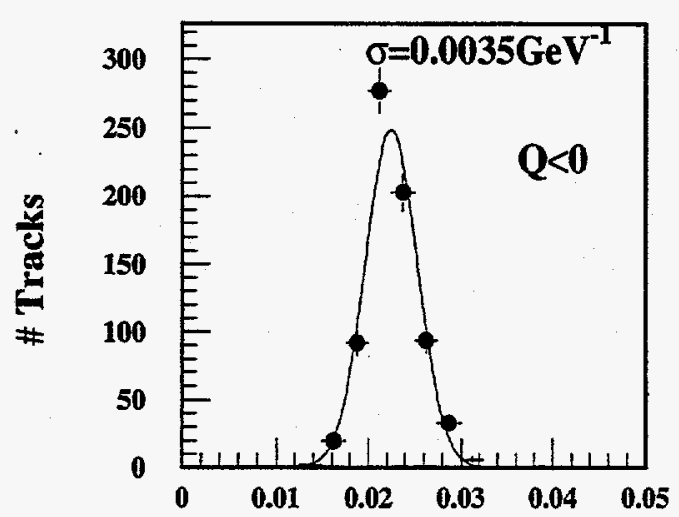

Inverse Momentum $\left(\mathrm{GeV}^{-1}\right)$

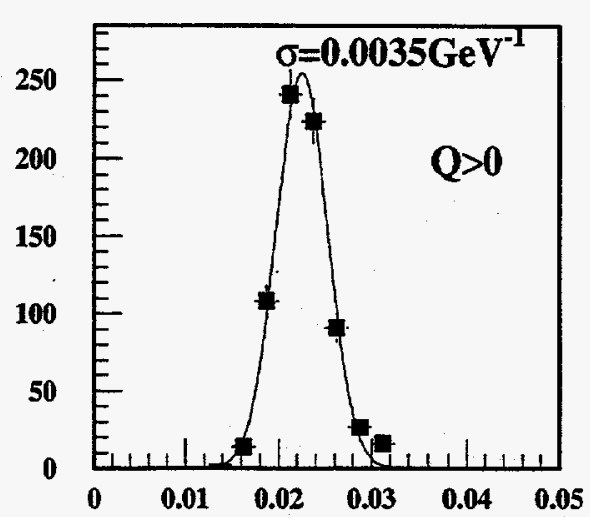

Inverse Momentum( $\left.\mathrm{GeV}^{-1}\right)$

Figure 3.19: Dip angle resolution and momentum resolution for $\mathrm{CDC}+\mathrm{VXD}$ tracks from $\mu^{+} \mu^{-}$events.

The $\chi^{2}$ distributions for the combined CDC+VXD are shown in Fig. 3.21. Included are the distributions for tracks that linked to two or more VXD hits and those that only linked to one hit. It is necessary to consider the two classes separately as a different recognition procedure is used for single hit links and since single hit links are more likely to be contaminated with background hits. The distributions agree 


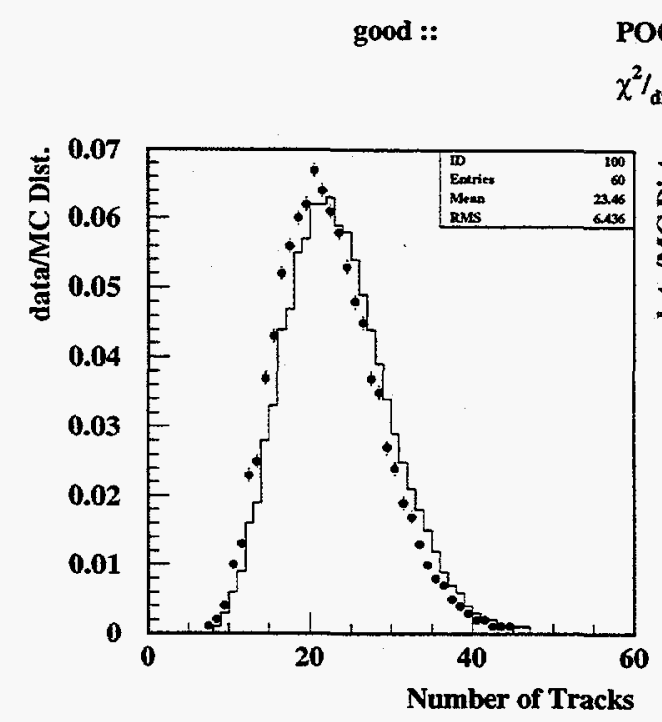

$\begin{array}{ll}\operatorname{POCA}_{\mathrm{z}}<\mathrm{cm} & \mathrm{P}_{\mathrm{xy}}>0.2 \mathrm{GeV} \\ \chi^{2} l_{\mathrm{dr}}<5 . & \cos \theta<0.8\end{array}$
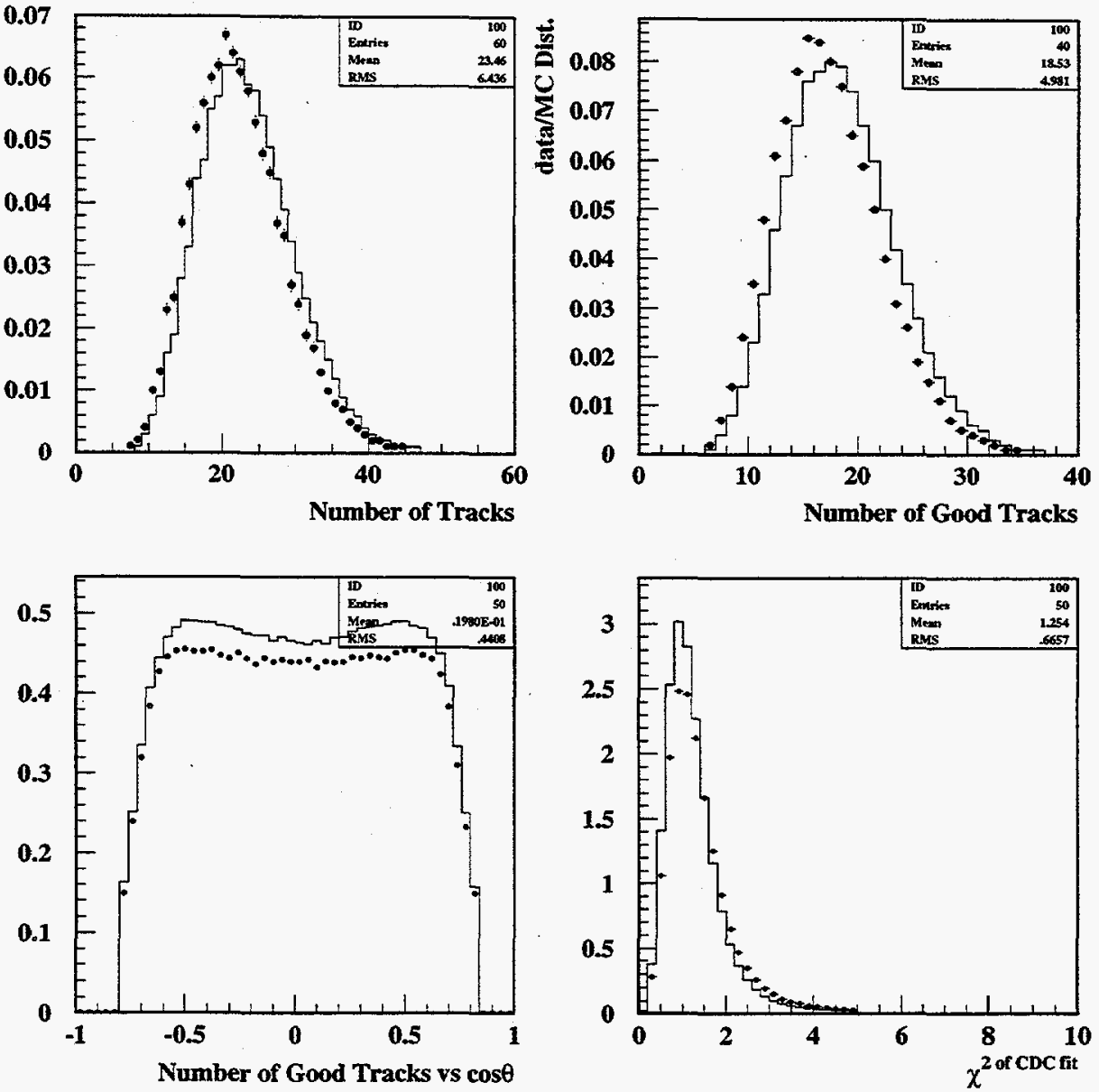

Figure 3.20: Distributions of variables indicative of the tracking performance for the CDC.

well for both classes.

The number of vertex hits per track distribution shown in Fig. 3.21 is a sensitive measure of the VXD alignment and efficiency of the CCDs. A small difference between the number of single hit links and number of multihit links per track is observed and has been identified as a small difference in the radial position of the ladders in the Monte Carlo simulation from the actual radii.

The number of CDC hits per track indicates any problems with hit efficiency in the chamber. The effects of pulse height cuts, frequency of dead layers, and inefficiencies 

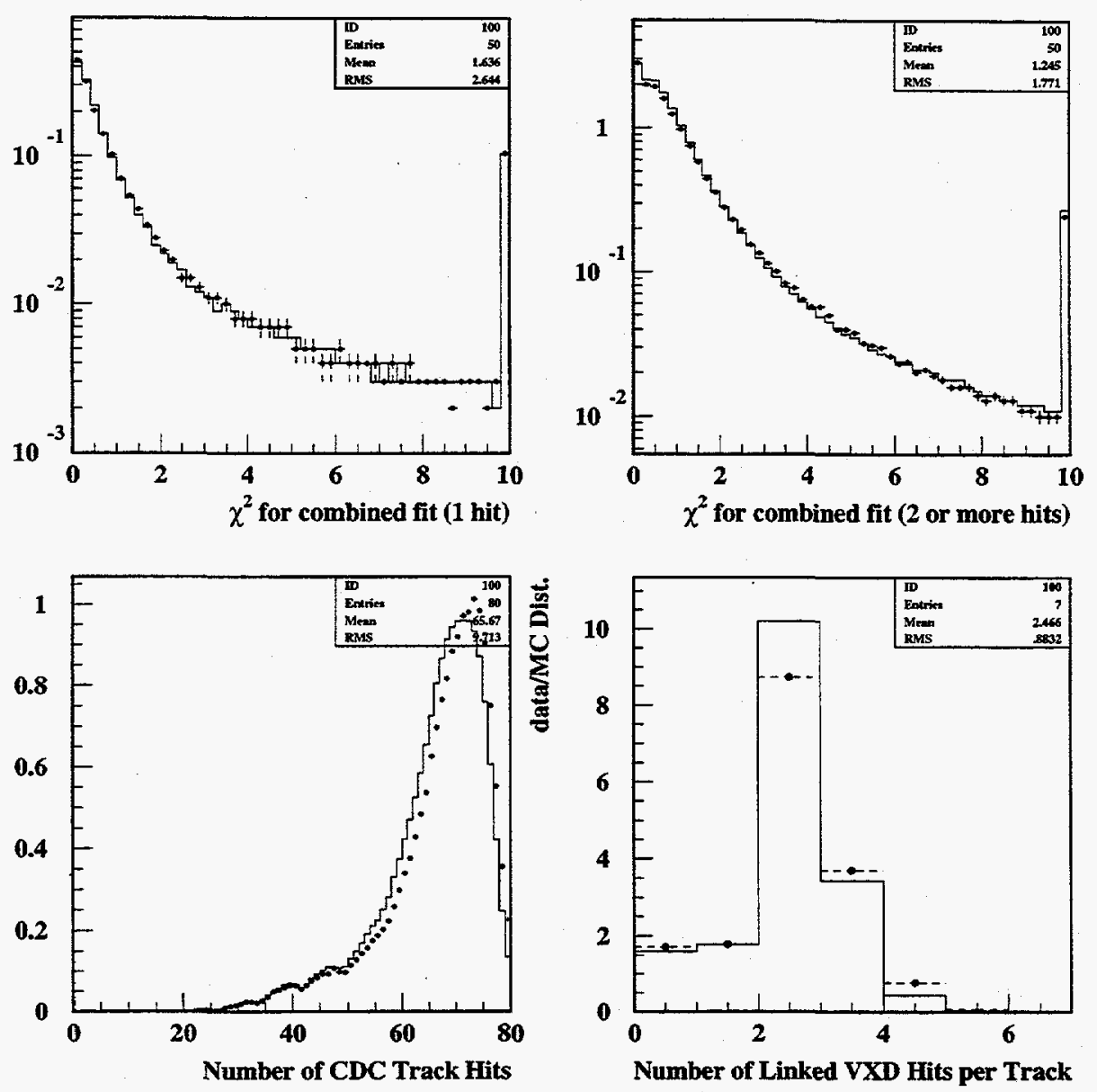

Figure 3.21: Distributions of variables indicative of the tracking performance for the CDC and VXD.

near ends of wires are reflected in these distributions. The discrepancy between Monte Carlo and data for large number of track hits will not significantly affect the analyses as the cut will be at 40 hits and the difference in precision from tracks of various but high number of hits is negligible. The effects of the pulse height cut is most noticeable in the $\cos \theta$ distribution of the number of hits per track. The cut is responsible for the dip near $\cos \theta=0$. shown in Fig. 3.20 . 


\section{Chapter 4}

\section{Primary Vertex Position Measurement}

Knowledge of the primary vertex position is critical to the analyses of this thesis. To have a pure $b$-tag it is necessary to

- clearly distinguish the primary vertex from other vertices

- measure the primary vertex position with a precision comparable to the impact parameter resolutions

The beam-beam overlap is only a few microns in each of the transverse dimensions. The SLC beam position monitoring indicates that the location of this overlap is stable to within $6 \mu \mathrm{m}$ in each of the transverse dimensions over a period of at least $30 Z^{0}$ events. In contrast, the longitudinal spread of the interaction region is $\sim 700 \mu \mathrm{m}$ due to the bunch length. This spread in the interaction region is considerably better than, for instance, the environment for the LEP detectors. LEP detectors typically have a $\sim 30 \mu \mathrm{m} r \phi$ impact resolution and a luminous region of $\sim 215 \mu \mathrm{m} \times \sim 200 \mu \mathrm{m}$ in $r \phi$ and $1.0 \mathrm{~cm}$ in $z$. The difference in the transverse and longitudinal spread of the interaction region in addition to the difference in tracking resolution in these dimensions leads to the use of separate methods for measuring the components of the primary vertex position. The methods described below minimize the occurrence of 

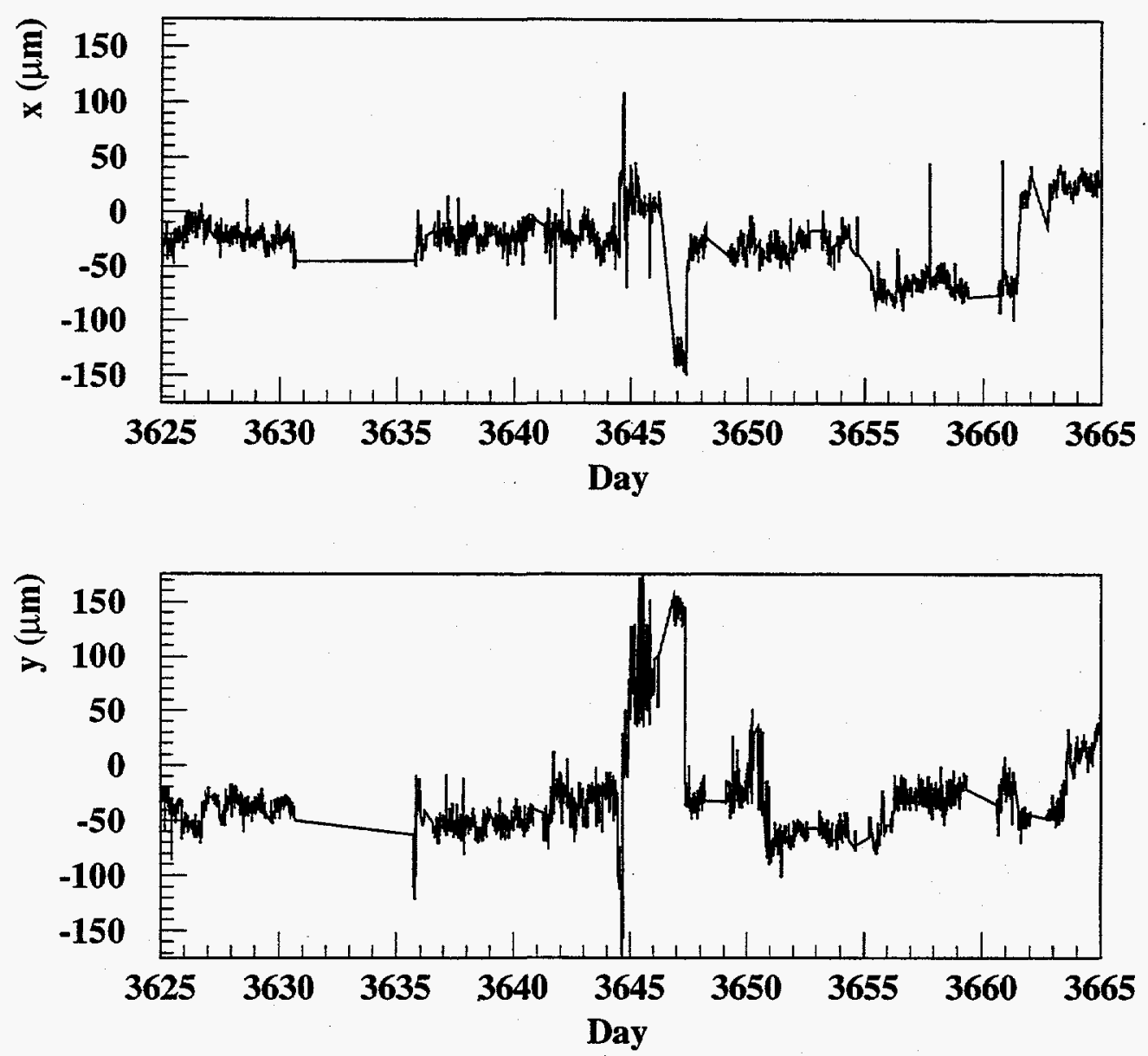

Figure 4.1: Beam positions for the 1994 run as determined from the currents applied to the corrector magnets, located near the interaction point, to keep the $e^{+} e^{-}$beams in collision.

accidentally identifying a $b$-decay vertex, which may be the source of a large fraction of the tracks in an event, as a primary vertex. 


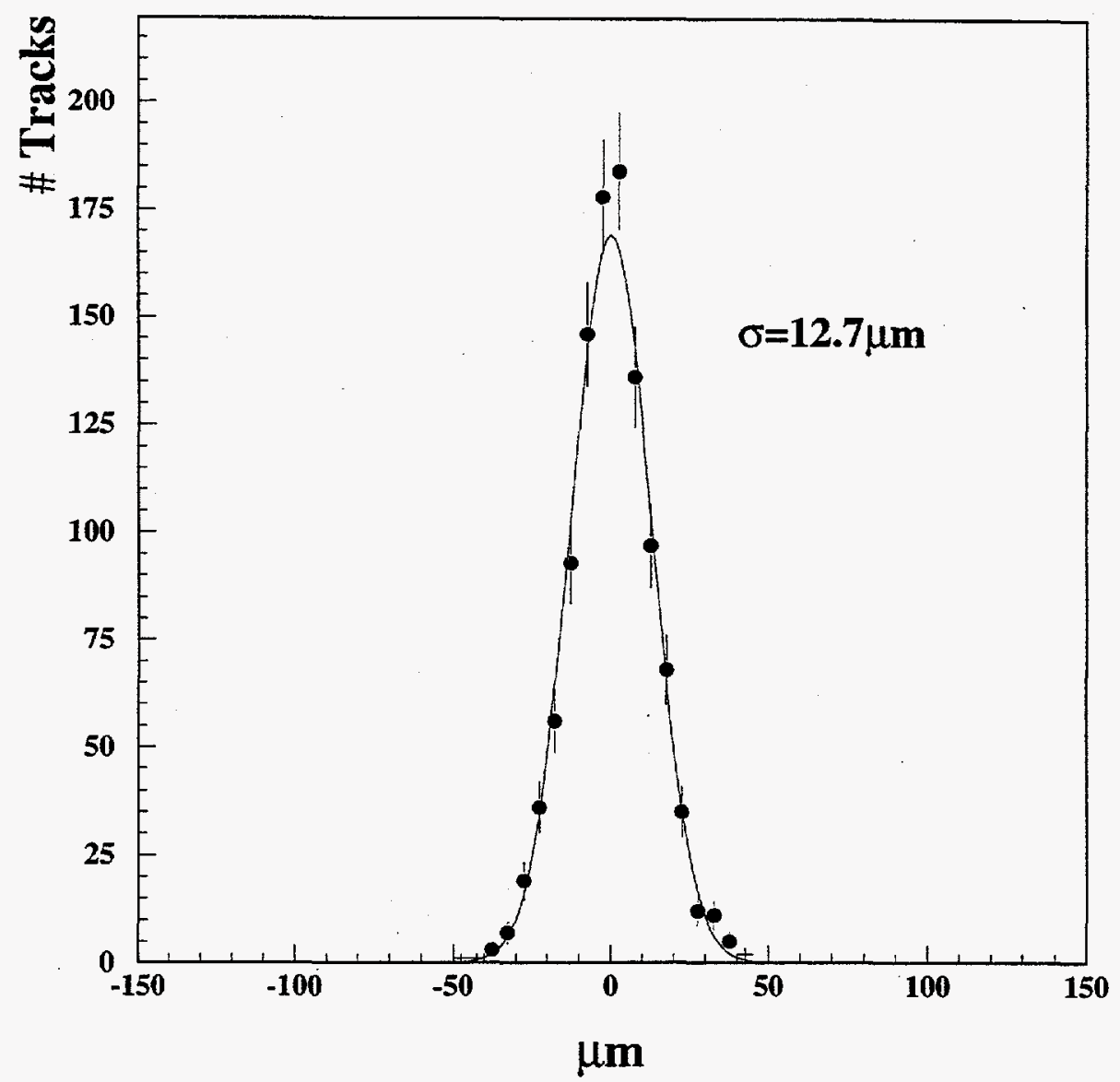

Figure 4.2: $x y$ impact parameters of tracks in $\mu$ pair events with respect to the measured primary vertex position determined independently by hadronic $Z^{0}$ events.

\subsubsection{Determining Transverse Components of the Primary Vertex}

The procedure uses hadronic events for the position measurements along with crosschecks from beam position information provided by SLC and leptonic events[30][23]. In the $r \phi$ plane information on the stability of the beams is provided by the feedback system used to keep the beams in collision. Specifically, the current needed to be applied to the corrector magnets to keep the beams in collision provides information on the relative location of the beams. This is useful both for determining the 
amount of fluctuation in the beam position and for identifying when a significant change $(\sim 100 \mu \mathrm{m})$ in the position has occurred. Such large changes result from either realignment of the magnets, some times necessary to reduce backgrounds, other accelerator problems, or when the accelerator is recovering from a long period of nonoperation. The beam positions from the feedback systems are shown for a period of 40 days in Fig. 4.1. The information from the feedback systems suggest that the $r \phi$ position of the luminous region is stable to within $6 \mu \mathrm{m}$ over the period of many $Z^{0}$ events. Therefore, instead of measuring the position event to event which would have the disadvantage of having large errors along the direction of the events energy flow and high probability of incorrectly selecting a secondary vertex for a primary one, the tracks from $\sim 30$ hadronic events are used. Tracks within $3 \sigma$ to a trial $x y$ position are fit to a common vertex. The fitted position is then used as the new trial position and the process is repeated. The $\chi^{2} / d f$ for the fit is required to be less than 1.3.

To test the accuracy of the measured position, impact parameters of tracks in $\mu^{+} \mu^{-}$events are studied. Since these events are not used in finding the position this yields a completely independent check. The distribution of the impacts is shown in Fig. 4.2. Part of the width is due to tracking resolution. To determine the tracking resolution contribution, the resolution measured from the miss distance of the $\mu^{+} \mu^{-}$ tracks is used (see Fig. 5.3). After applying this correction, an uncertainty in the $x$ and $y$ position of the primary of $7 \mu \mathrm{m} \pm 2 \mu \mathrm{m}$ is obtained[23].

The Monte Carlo generates primary vertex positions that are distributed as a Gaussian. When the primary vertex position measurement is performed on the Monte Carlo the tails on the impact distributions are observed to be negligible. Tails are observed in a small fraction of the data events. The parameters given in Table 4.1 were found to accurately describe the tails.

\subsubsection{Determining the Longitudinal Component of the Pri- mary Vertex}

The larger spread of the luminous region in the longitudinal direction necessitates that an event by event measure of the longitudinal component be made. It was found that 


\begin{tabular}{|c|c|c|}
\hline Year & Gaussian width of tail & Fraction of events affected \\
\hline 1992 & $100 \mu \mathrm{m}$ & $0.25 \%$ \\
1993 & $100 \mu \mathrm{m}$ & $0.50 \%$ \\
1994 & $100 \mu \mathrm{m}$ & $0.50 \%$ \\
\hline
\end{tabular}

Table 4.1: measured $x y$ primary vertex position tails.

the best procedure for locating the longitudinal position of the primary vertex is to use the median $z$ of tracks[23]. Each CDC+VXD track is extrapolated to the point of closest approach to the measured primary vertex position in the dimension transverse to the beam. Then the median of the $z$ position of those tracks which have an $x y$ distance of closest approach with respect to the measured primary vertex position less than $500 \mu \mathrm{m}$ and less than $3 \sigma$ of its uncertainty are taken as the longitudinal position of the primary vertex. If no $\mathrm{CDC}+\mathrm{VXD}$ tracks pass these cuts then all CDC+VXD tracks are used. The resolution is determined from the Monte Carlo to be $38 \mu \mathrm{m}$. 


\section{Chapter 5}

\section{Tagging Variables}

Each analysis uses a property of the tracks(a tag variable) to form a quantity that can be cut on to tag $b$-decays. For the 2-D Impact Parameter Event Tag, the tag variable is the $r \phi$ normalized impact parameter of the tracks with respect to the $r \phi$ primary vertex position. For the Lifetime Probability Tag, the minimized 3-D normalized impact parameter is used. The advantage of each and how they are calculated and modified to enhance their use for $b$-tagging is described in the following sections.

\subsection{The 2-D Impact Parameter}

The 2-D impact parameter is the distance of closest approach between the trajectory of a charged particle, measured by the CDC and VXD, extrapolated to its point of closest approach to the measured primary vertex position as seen in the $r \phi$ plane. The 2-D Impact Parameter Event Tag uses this to take advantage of the high precision of the detector and the accurate knowledge of the primary vertex position in the $r \phi$ plane. The lifetime $(\tau)$ of the particle which decayed to produce the observed track is related to the tracks 2-D impact parameter $(\delta)$ by Eqn. 5.1 .

$$
\langle\delta\rangle=\langle\beta \gamma c \tau \sin \psi \sin \theta\rangle \propto c \tau
$$

Here $\psi$ is the angle between the parent particle decay direction and the direction of the particle in the $r \phi$ plane, and $\theta$ is the polar angle. As described below, the 
impact parameter may be signed in such a way as to allow the resolution and lifetime information of the impact parameters to be separated.

\subsubsection{Impact Parameter Signing}

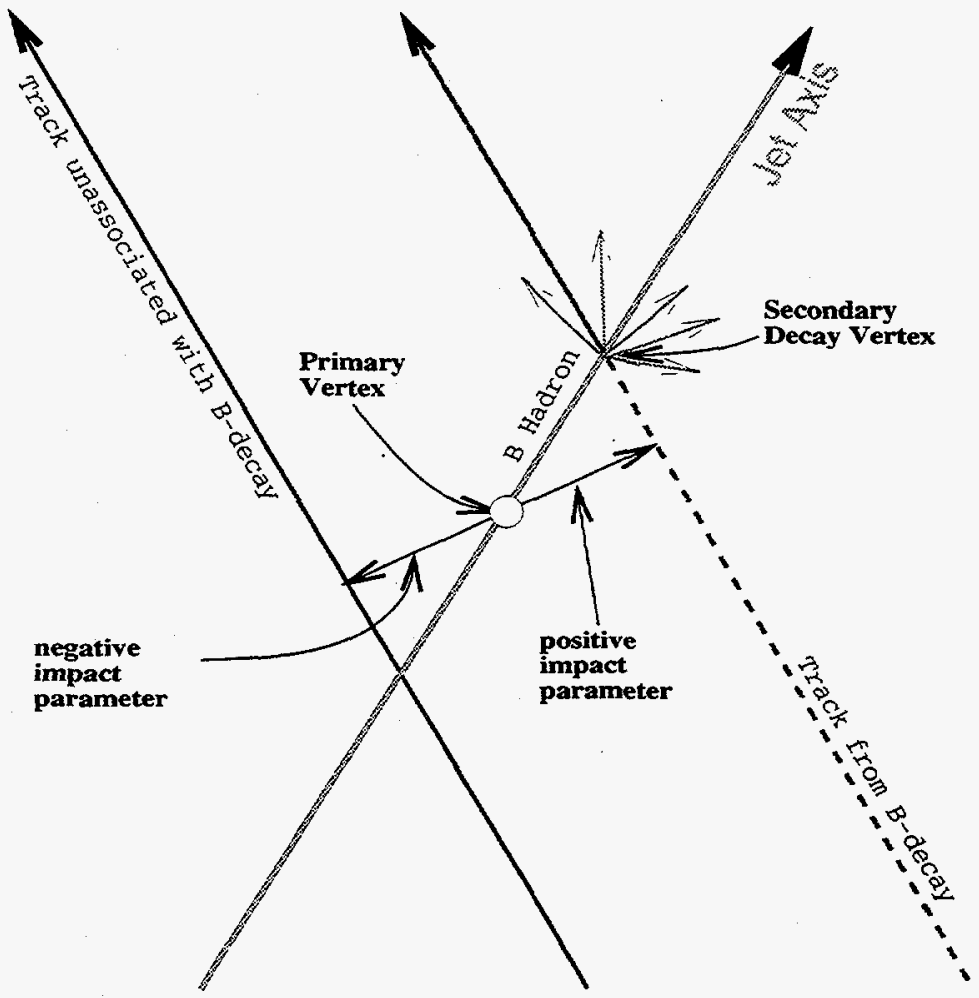

Figure 5.1: Drawing illustrating the signing of the impact parameters. Tracks which appear to have come from decays in the direction opposite to the associated jet are assigned negative impact parameters. Tracks from decays of particles with long lifetimes should have positive impact parameters.

The 2-D impact parameter is signed positive if the associated track appears to have originated in front of the measured primary vertex position along the decay direction of the $B$-hadron. Otherwise, it receives a negative sign. This is done by giving the impact parameter the sign of the dot product of the measured primary vertex position to the point of closest approach direction with the jet direction in the $r \phi$ plane. An example of this is shown in. Fig. 5.1. The jets are identified using the JADE[49] jet finding algorithm with $y_{\text {cut }}=0.02$. At this cut, an average of 2.5 jets per $Z^{0}$ event 
are found. The value of $y_{\text {cut }}$ was chosen to maximize the fraction of tracks from the $B$-decay that are signed correctly. This is shown in Fig. 5.2. Only positive impact parameters will be used in the tag but the negative impact parameters allow the impact parameter resolution to be calibrated with most of the tracks from long lived particle decay products removed. The means by which the latter is done is described in Sect. 7.4.

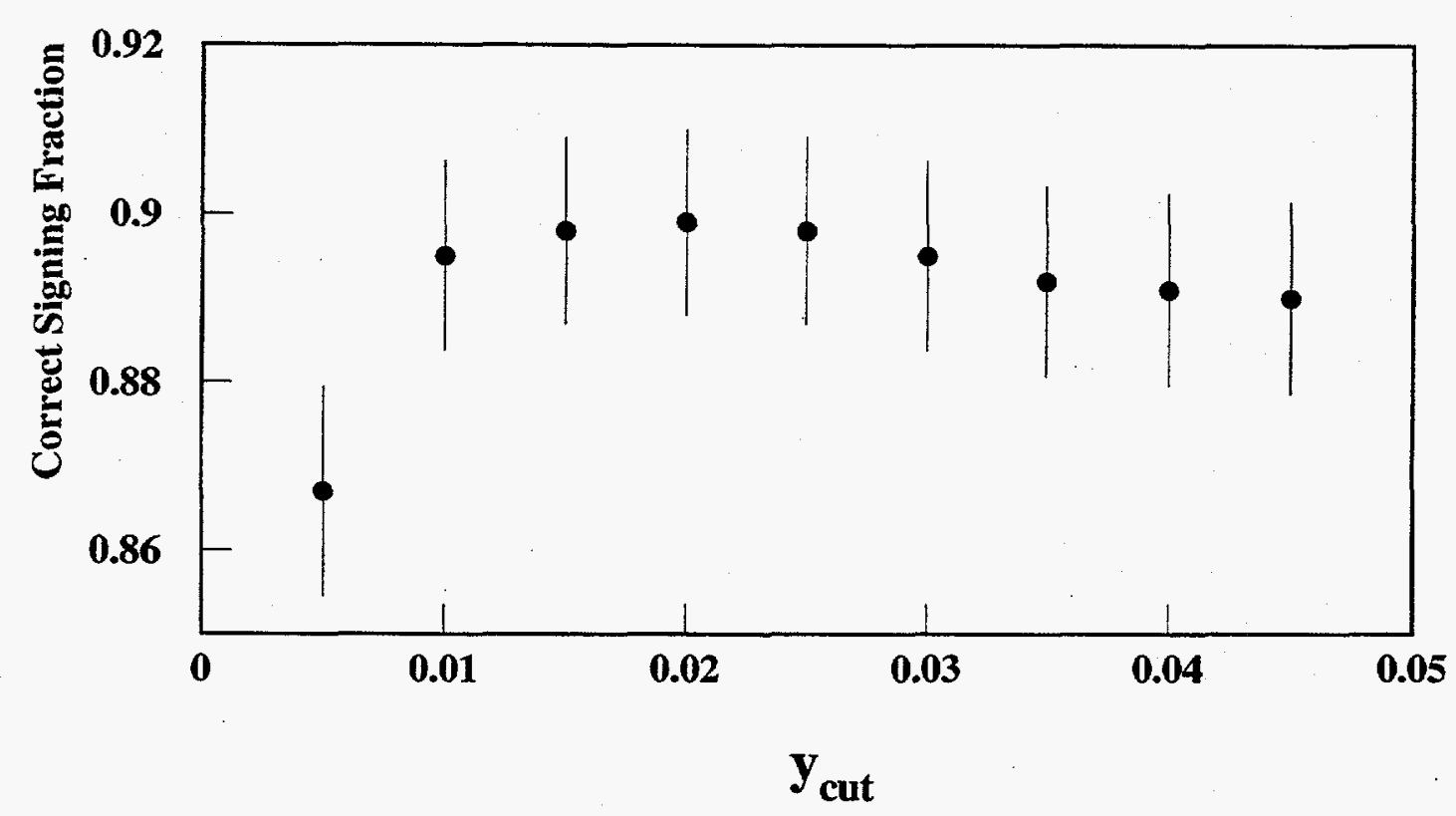

Figure 5.2: Fraction of $B$-decay tracks correctly signed versus $y_{\text {cut }}$.

\subsubsection{Normalizing the Impact Parameter}

Large impact parameters frequently result from poorly fitted tracks. Furthermore, the amount of multiple scattering and precision of the tracking varies significantly with the dip angle of the track. Therefore, it is best to use a tagging parameter that indicates the significance of an impact parameter with respect to its uncertainties. The quantity representing the significance of a tracks $r \phi$ impact parameter is called 
the $r \phi$ normalized impact parameter $\left(\delta_{\text {norm }}\right)$. It is obtained by dividing the impact parameter $(\delta)$ by the sum of the uncertainty on the track impact parameter and the uncertainty on the $x y$ position of the primary vertex added in quadrature $\left(\sigma_{\delta}^{n e t}\right)$.

\subsubsection{Contributions to the Impact Parameter Uncertainty}

The uncertainty in the $r \phi$ impact parameter contains contributions from the intrinsic resolution of the tracking devices, the scattering of the particle as it passes through the SLD material, and the uncertainty in the primary vertex position measurement. These contributions combine in the manner shown in Eqn. 5.2[51].

$$
\sigma_{\delta}=\sqrt{\left(\sigma_{\delta}^{\text {intrinsic }}\right)^{2}+\left(\sigma_{\delta}^{\text {primary vertex }}\right)^{2}+\left(\frac{\sigma_{\delta}^{\text {scattering }}}{p_{t} \times \sqrt{\sin \theta}}\right)^{2}}
$$

The contribution from the transverse primary vertex position measurement is discussed in Chapter 4. The intrinsic and multiple scattering contributions are described below.

\subsubsection{Intrinsic Detector Resolution}

The intrinsic resolution is the uncertainty due to detector resolution on the position of track hits contributing to the track fit. For a pair of hits with precision $\sigma$ at distance $r_{1}$ and $r_{2}$ from the primary vertex position with distance $\delta r$ between them, the intrinsic resolution is given by:

$$
\sigma_{\delta}^{\text {intrinsic }}=\frac{r_{1} \times \sigma}{\delta_{r}} \oplus \frac{r_{2} \times \sigma}{\delta_{r}}
$$

By having the inner layer of the VXD close to the beam-line (small $r_{i}$ ) this uncertainty is reduced compared to ALEPH whose minivertex detector has an inner radius of $63 \mathrm{~mm}$. However, the VXD suffers from a small lever $\operatorname{arm}\left(\delta_{r}\right)$. A comparison of the intrinsic impact parameter resolutions, between SLD and ALEPH, is shown in Table 5.1 .

The intrinsic resolution is measured using the miss distance of tracks in $\mu^{+} \mu^{-}$ 


\begin{tabular}{|l|c|c|}
\hline Detector & $\sigma_{r \phi}^{\text {intrinsic }}$ & $\sigma_{r z}^{\text {intrinsic }}$ \\
\hline SLD & $11 \mu \mathrm{m}$ & $38 \mu \mathrm{m}$ \\
ALEPH & $25 \mu \mathrm{m}$ & $30 \mu \mathrm{m}$ \\
\hline
\end{tabular}

Table 5.1: SLD and ALEPH intrinsic impact resolutions. [50]

events. Due to the high energy of these tracks $(45 \mathrm{GeV})$ the multiple scattering contribution to the impact uncertainty is negligible. Since the primary vertex position is not used, the uncertainty coming from this is removed. The $\mu^{ \pm}$tracks should each originate from the primary vertex so any difference between the measured origins of the tracks is attributable to tracking resolution. The distribution of the miss distance is shown in Fig. 5.3. The width of the $r \phi(r z)$ miss distance distribution is $16 \mu \mathrm{m}(52 \mu \mathrm{m})$. This corresponds to a resolution of $11 \mu \mathrm{m}$ in the $r \phi$ plane and $37 \mu \mathrm{m}$ in the $r z$ plane for the impact of each track.

\subsubsection{Multiple Scattering}

As a particle passes through the material of the beam pipe, vertex detector, cryostat and $\mathrm{CDC}$ it suffers small angle scattering from Coulomb interactions with the nuclei. It is necessary to determine the mean scattering angle and number of scatters experienced by the particle as it traverses through each layer of material. The problem is complicated by varying thicknesses and mixtures of materials. Initial attempts at using Gaussian approximations resulted in significant discrepancies in both the core and tails of the $r \phi$ impact parameter distributions. A modified Moliere scattering procedure [31] is now used and as will be shown in later chapters it is a remarkably good model of both the core and tails of the impact parameter distribution. The model uses Kummers hypergeometric functions with inputs of the particle charge and energy, material density and molecular weight, and the total path length through the scattering medium. The approximate uncertainty in the impacts introduced by a particle passing through several layers $(i)$ of material with mean $\operatorname{radii}\left(R_{i}\right)$ and thickness $\left(L / L_{R}\right)_{i}$ is given in Eqn. 5.4. 

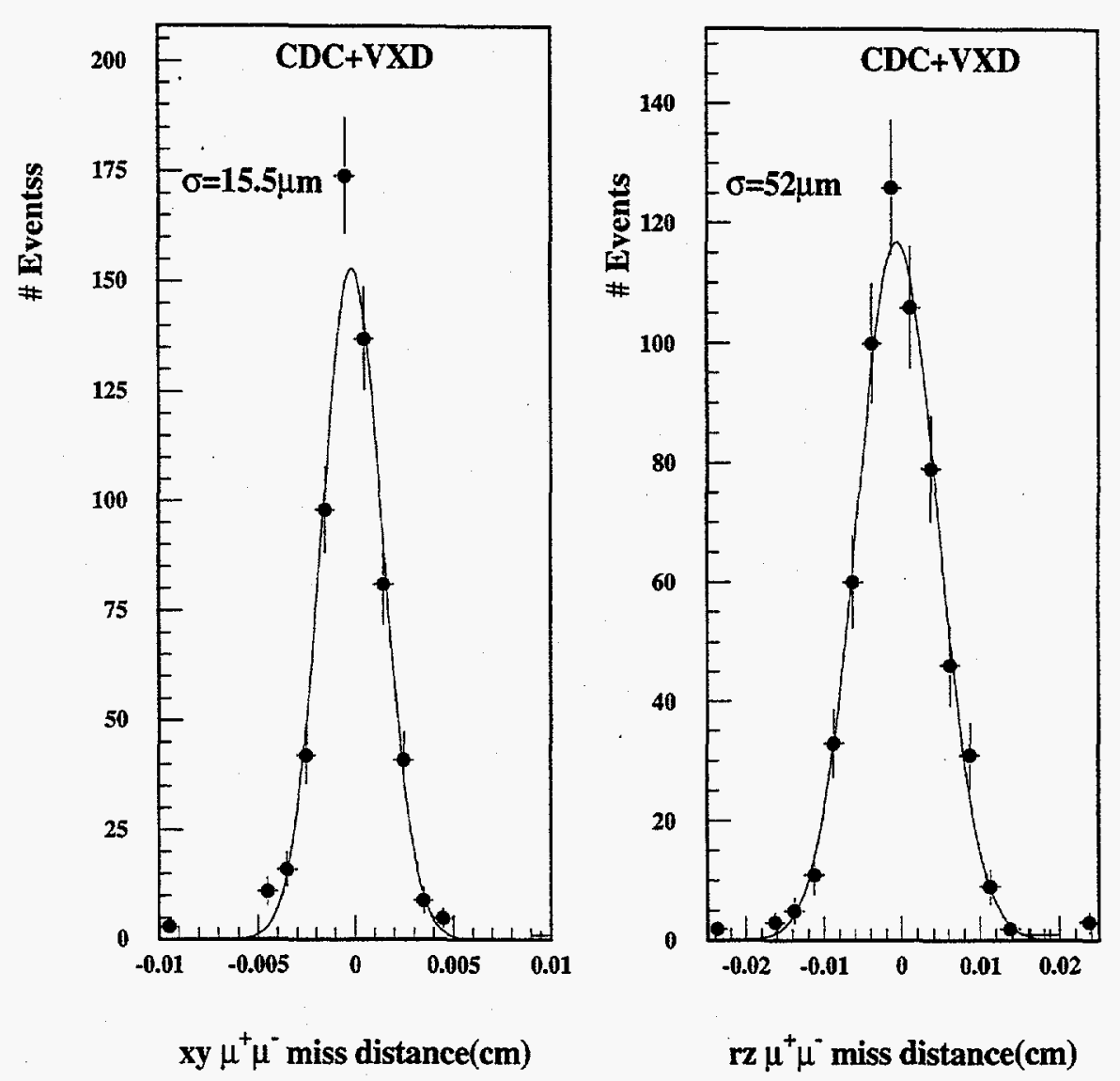

Figure 5.3: Miss distance of tracks in $\mu^{+} \mu^{-}$events.

$$
\sigma_{\delta}^{m s c t}=\frac{0.015}{p \times \sin ^{3 / 2} \theta} \sum R_{i} \sqrt{\left(L / L_{R}\right)_{i}}
$$

The radii and thicknesses of material traversed by a particle passing through the SLD at $\cos \theta=0$ are given in Table 5.2. A track will typically intersect 2.3 CCDs. When a particle reaches the outside of the VXD it will have traversed an average $L / L_{R}$ of $3.5 \%$. The ALEPH minivertex detector has significantly thicker ladders(wafers). They are $300 \mu \mathrm{m}$ thick. When a particle reaches the outside of their detector it has traversed an average of $4.4 \%$ radiation lengths including the beam-pipe[50]. 


\begin{tabular}{|c|r|r|}
\hline & $\begin{array}{r}\text { Mean Radius } \\
(\mathrm{mm})\end{array}$ & $\begin{array}{r}L / L_{R} \\
(\%)\end{array}$ \\
\hline Ti Liner & 25.0 & 0.28 \\
Be Beam Pipe & 25.5 & 0.28 \\
Be Inner Shell & 27.0 & 0.14 \\
Layer 1 Ladder & 29.5 & 1.15 \\
Layer 2 Ladder & 33.5 & 1.15 \\
Layer 3 Ladder & 37.5 & 1.15 \\
Layer 4 Ladder & 41.5 & 1.15 \\
Be Outer Shell & 45.5 & 0.20 \\
N2 Gas & 80.0 & 0.06 \\
Cryostat & 165.5 & 0.98 \\
CDC Inner Wall & 200.0 & 1.80 \\
CDC Gas & 600.0 & 0.50 \\
CDC Wires & 600.0 & 0.20 \\
\hline
\end{tabular}

Table 5.2: Radiation lengths seen by a track passing through the central tracking volume at $\theta=90^{\circ}$.

\subsubsection{Parameterization of Impact Uncertainty}

The intrinsic and multiple scattering contributions to the impact parameter uncertainties, discussed above, yield the uncertainty from tracking alone shown in Eqn. 5.5. Here, $p_{t}$ is the momentum transverse to the beam-line. The momentum dependence of the $r \phi$ and $r z$ impact parameter uncertainties for a track at $\cos \theta=90^{\circ}$ is shown in Fig. 5.4.

$$
\begin{aligned}
& \sigma_{\delta}^{r \phi}=11 \mu \mathrm{m} \oplus \frac{80 \mu \mathrm{m}}{p_{t} \sin \theta} \\
& \sigma_{\delta}^{r z}=38 \mu \mathrm{m} \oplus \frac{70 \mu \mathrm{m}}{p_{t} \sin \theta}
\end{aligned}
$$

The $r \phi$ impact parameter, $r \phi$ normalized impact parameter and $r \phi$ impact parameter uncertainty(not including primary vertex position contribution) are shown in Fig. 5.5 for several ranges in momentum transverse to the beam-line. These show the decreasing influence of multiple scattering as the momentum increases and the constant core 


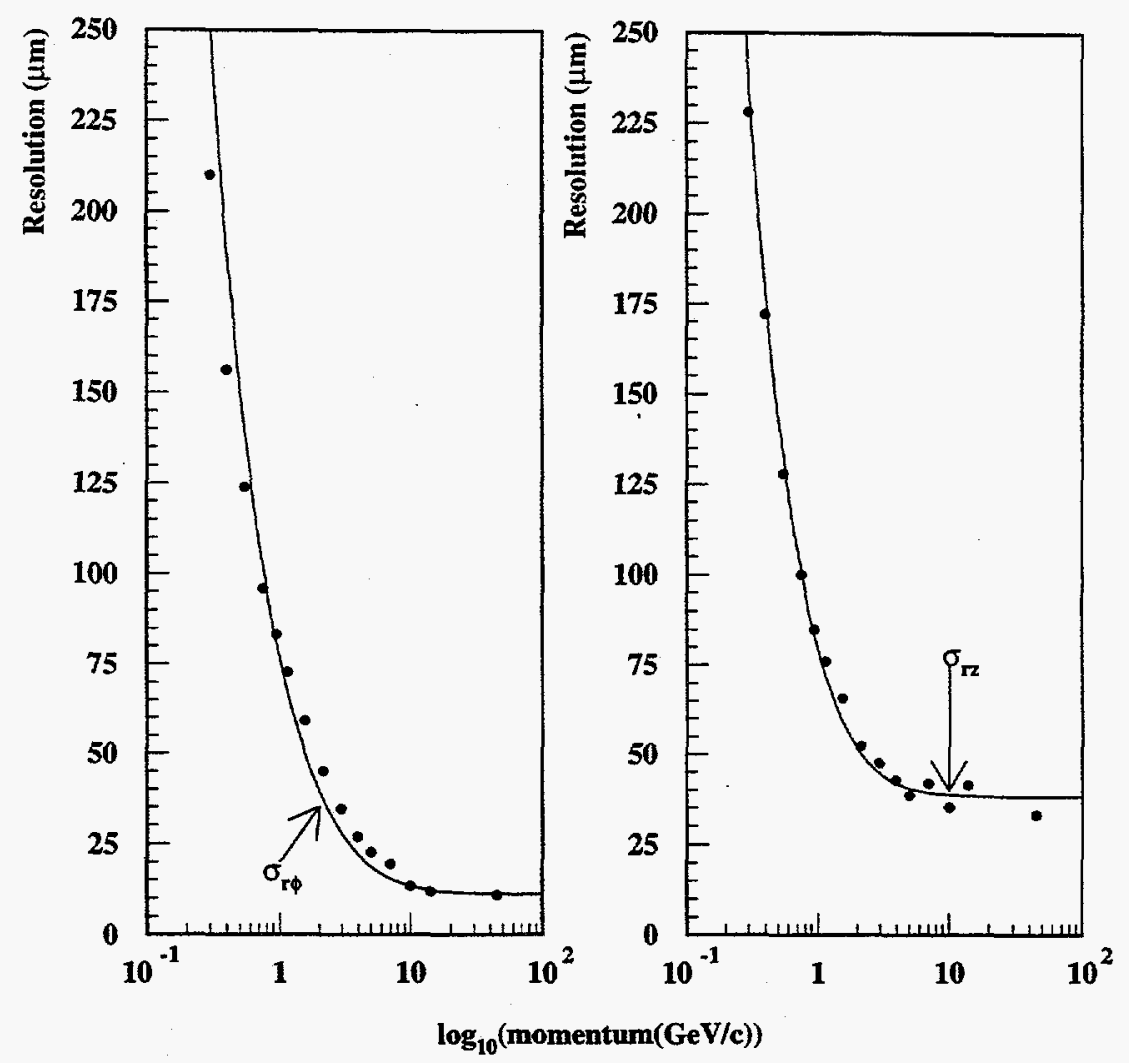

Figure 5.4: $r \phi$ (solid) and $r z$ (dashed) impact parameter resolutions vs. momentum for tracks at $\cos \theta=90^{\circ}$.

width of the normalized distribution.

\subsection{The Minimized 3-D Impact Parameter $(\chi)$}

For the Hemisphere Tag, the tag variable is the minimized 3-D normalized impact parameter, henceforth called $\chi$. It is effectively the minimal value obtained on the track trajectory for the quantity

$$
\delta^{3 D}=\sqrt{\left(\frac{x-x_{P V}}{\sigma_{x}}\right)^{2}+\left(\frac{y-y_{P V}}{\sigma_{y}}\right)^{2}+\left(\frac{z-z_{P V}}{\sigma_{z}}\right)^{2}}
$$

where $(x, y, z)$ represent a position on the track trajectory, $(x, y, z)_{P V}$ represent the measured primary vertex position and $\sigma_{x, y, z}$ are the impact parameter uncertainties. 
Similarly to the $r \phi$ impact parameters shown in Fig. 5.5, one can see the extra lifetime information in the $r z$ dimension in Fig. 5.6 that is employed by this variable. Using the full 3-D information makes the tag variable a more sensitive measure of the lifetime. In this section, the procedure by which $\chi$ is determined, and the signing of

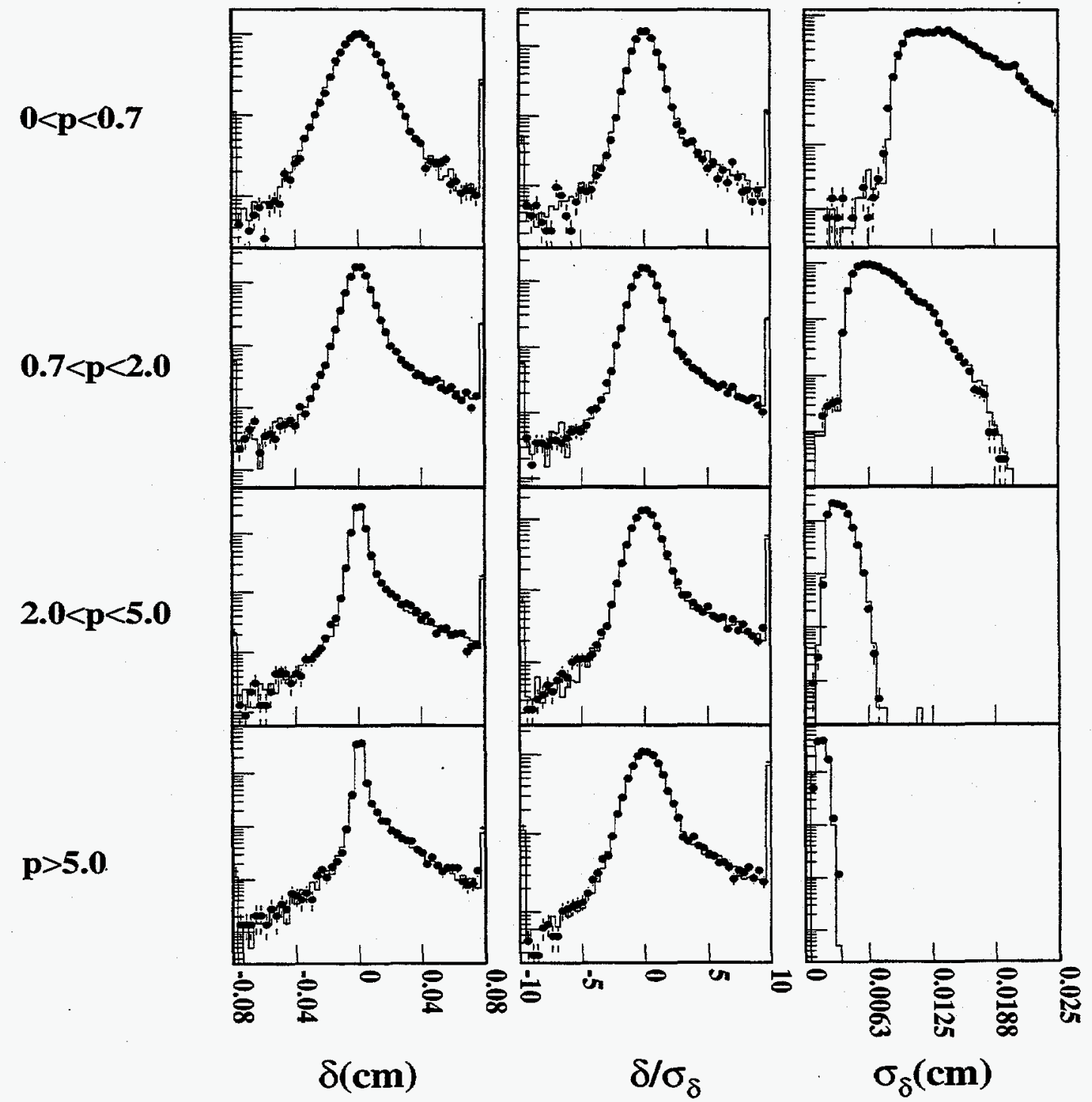

Figure 5.5: The 2-D $r \phi$ impact parameter $(\delta), 2$-D $r \phi$ normalized impact parameter $\left(\delta / \sigma_{\text {delt }}\right)$ and 2-D $r \phi$ impact parameter uncertainty $\left(\sigma_{\text {delta }}\right)$. The data is represented by the points and the Monte Carlo by the histogram. 
$\chi$ will be described.

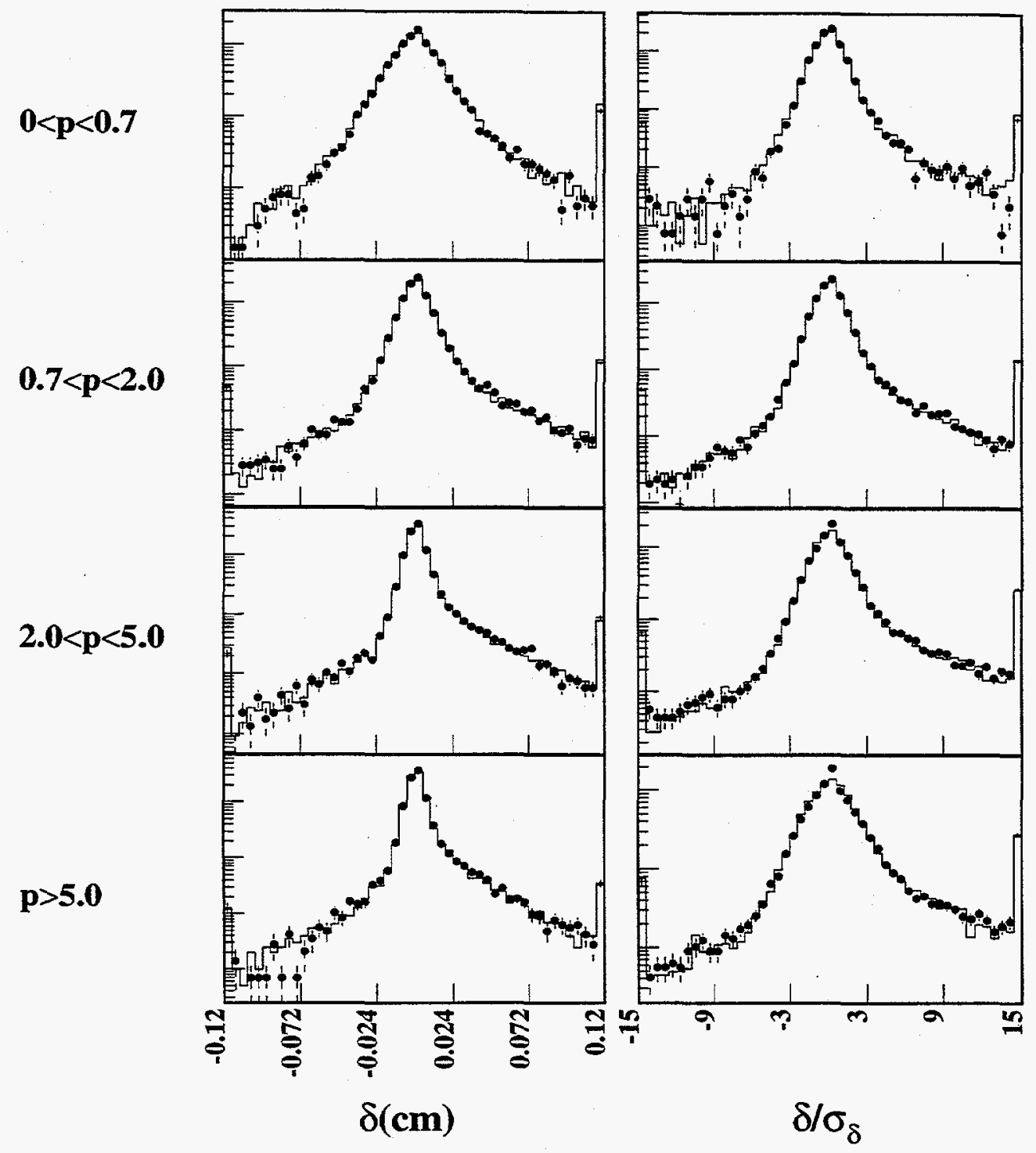

Figure 5.6: The 2-D $r z$ impact parameter $(\delta), 2-\mathrm{D} r z$ normalized impact parameter $\left(\delta / \sigma_{\text {delta }}\right)$. The data is presented the points and the Monte Carlo by the histogram.

The tag variable, $\chi$, is the contribution to the fit quality of a track from adding the primary vertex as an extra track hit with its corresponding position uncertainties. The uncertainty in the primary vertex position was determined from the individual procedures for measuring the $x y$ and $z$ of the primary vertex position. As described 


$$
\begin{aligned}
& x_{0}=\varphi=\text { azimuthal angle of momentum vector } \\
& x_{1}=k=1 / p_{\perp}=\text { curvature } \\
& x_{2}=s=\tan (\lambda)=\text { tangent of dip angle } \\
& x_{3}=\xi=\left(P O C A_{x}-P V_{x}\right) \sin \phi-\left(P O C A_{y}-P V_{y}\right) \cos \phi \\
& x_{4}=\eta=\left(z \text { at } P O C A_{x y}-P V_{z}\right) \cos \lambda
\end{aligned}
$$

Table 5.3: Track helix parameter definitions.

in Sect. 4, the uncertainty was determined to be $\sigma_{P V}=(7 \mu m, 7 \mu m, 38 \mu m)$. This is then used to modify the error matrix from the CDC+VXD track fit as follows:

$$
\chi^{2}=(\vec{m}-\vec{x})^{\dagger} \times V_{n e w} \times(\vec{m}-\vec{x})+\left(\frac{\xi}{\sigma_{\xi, \xi}}\right)^{2}+\left(\frac{\eta}{\sigma_{\eta, \eta}}\right)^{2}
$$

where

- $\mathrm{x}=$ vector of new track parameters defined in Table 5.3.

- $\mathrm{m}=$ vector of original measured track parameters

- $V^{-1}=$ inverse of the original track error matrix

- $\sigma_{\xi, \xi}=$ variance of $\xi$ from uncertainty in measured primary vertex position

- $\sigma_{\eta, \eta}=$ variance of $\eta$ from uncertainty in measured primary vertex position

$P O C A_{i}$ and $P V_{i}$ are the $i$ th components of the $r \phi$ point of closest approach and primary vertex position respectively.

Next, the track parameters are recalculated using the new error matrix, which includes the primary vertex, by minimizing the $\chi^{2}$ with respect to each parameter as follows:

$$
\frac{\delta \chi^{2}}{\delta m}=0=m^{\dagger} V^{-1}-\left(x^{\dagger} V^{-1}+\xi / \sigma_{\xi, \xi}+\eta / \sigma_{\eta, \eta}\right)
$$

The new error matrix $V_{n e w}^{-1}$ is: $V^{-1}$ except 
- $V_{3,3 \text { new }}^{-1}=V_{3,3}^{-1}+1 / \sigma_{\xi, \xi}$

- $V_{4,4 \text { new }}^{-1}=V_{4,4}^{-1}+1 / \sigma_{\eta, \eta}$

The new track parameters are then obtained from:

$$
\vec{x}=V_{\text {new }} * V^{-1} \times \vec{m}
$$

Once this has been done the $\chi^{2}$ coming from the contribution of the primary vertex is calculated from Eqn. 5.6.

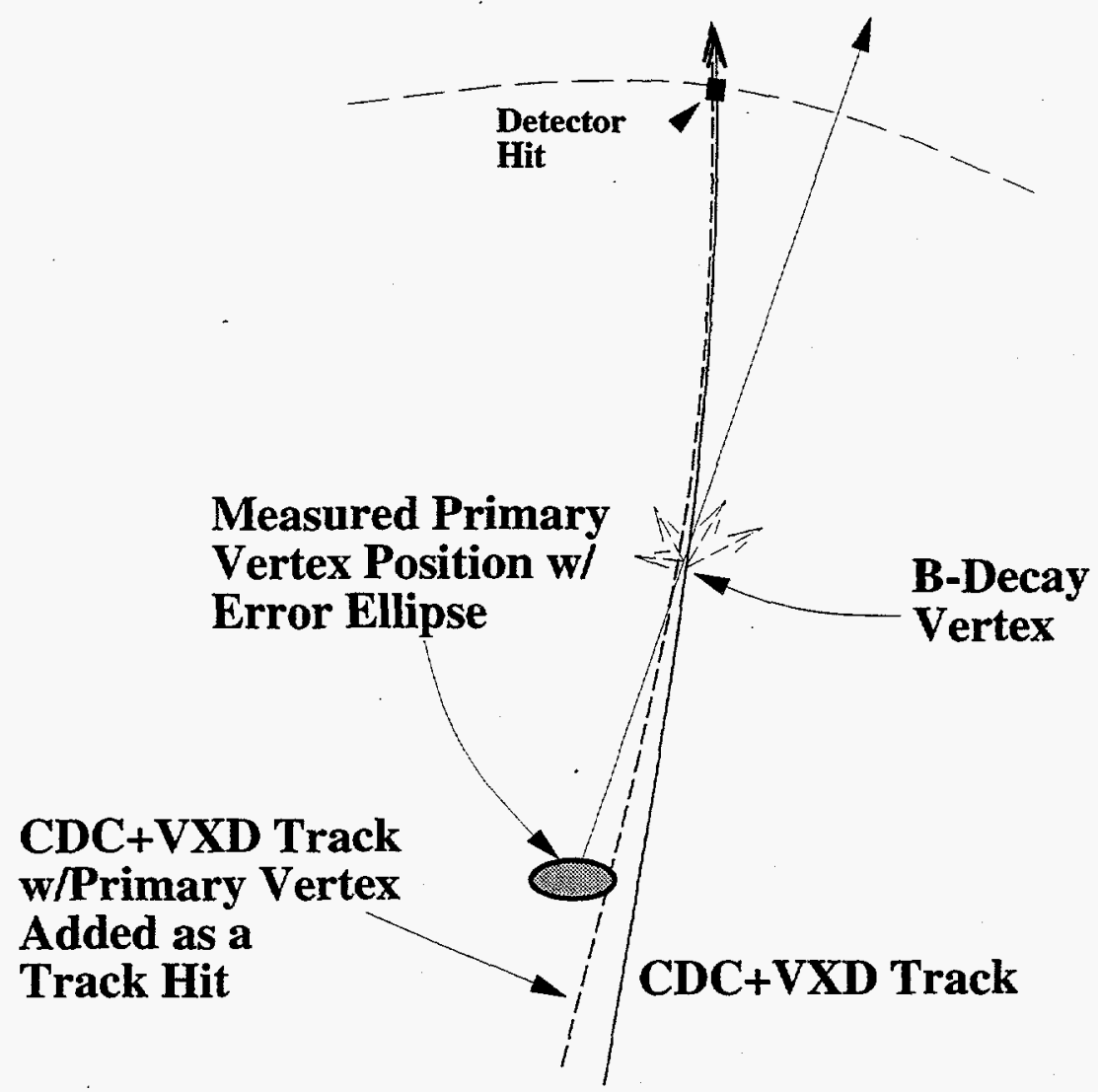

\subsubsection{Signing the Tag Variable}

As for the $r \phi$ impact parameter the variable representing the significance of the impact is signed to indicate whether it is likely to be associated with a long lived particle. An improved method, relative to the 2-D impact parameter signing, is used which 


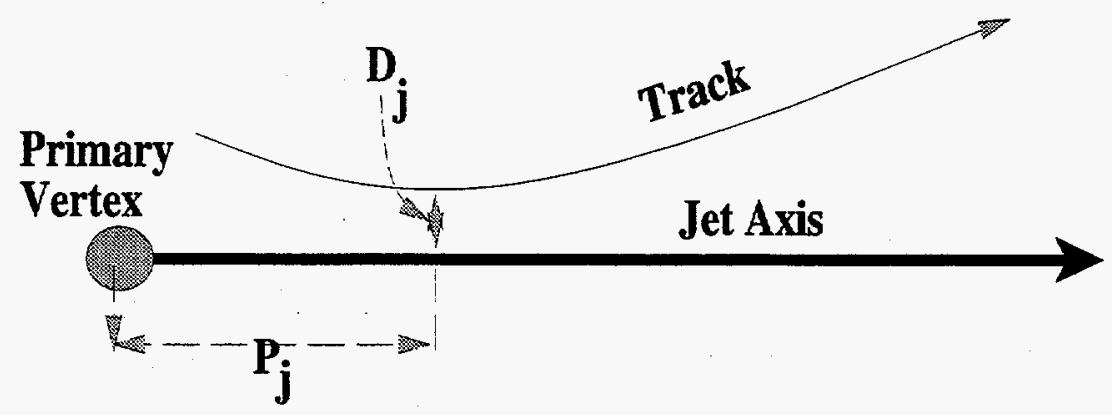

Figure 5.7: The signing of $\chi$.

includes information from the longitudinal dimension. The $\chi$ is signed by whether the track passes closest to the jet axis it is associated with in front(positive) or behind(negative) the primary vertex. This is the sign of $P_{j}$ in Fig. 5.7. The signed $\chi$ distributions for Monte Carlo and data are shown in Fig. 5.8. An obvious difference between these and the 2-D impact parameter distributions is the dip near $\chi=0.0$. This is due to phase space.

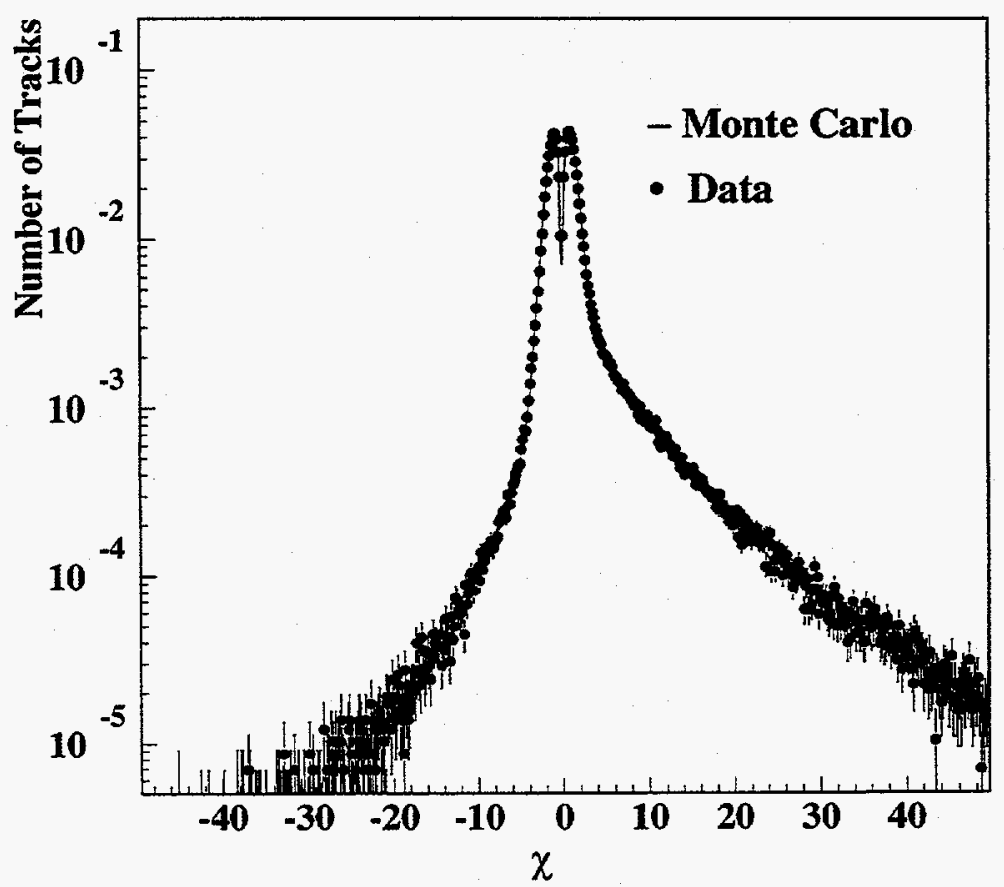

Figure 5.8: Data and Monte Carlo distributions of the tagging variable $\chi$. 


\section{Chapter 6}

\section{Physics Simulation}

Modified JETSET 6.3[32] and JETSET 7.4 generators are used for the simulation of $Z^{0} \rightarrow$ hadrons events. The Monte Carlo simulation is particularly important for the 2-D Impact Parameter Event Tag analysis where it is needed to accurately predict the tagging efficiencies for $b \vec{b}$ events. The Lifetime Probability Tag analysis will be most sensitive to the simulation of charm hadron decays. In this chapter, the means by which the decays of the $Z^{0}$ are simulated is described.

\subsection{Simulation of $B$-Hadron Decays}

For semileptonic $B$ decays the CLEO simulation is used in place of JETSET[23]. The CLEO simulation was found to better simulate the distribution of the lepton momentum in $B$ decays[33]. The CLEO model was further tuned [34][35] to obtain the excellent agreement with data shown in Fig. 6.1. Through tuning of the $D^{* *}$ production fraction and rate at which decays occurred by internal $W$ emission a good description of the charm hadron momentum spectrum in $B$ meson decays has been achieved[36]. The vector/pseudoscalar particle production ratios and strange quark yields in the fragmentation were also adjusted to get a good description of various measured particle production fractions and momentum spectra. The $D$ production spectrums are shown in Fig. 6.2. The distribution of the branching fraction into semileptonic, two body and $b \rightarrow c$ decays are given in Table 6.1 . 


\begin{tabular}{|l|c|l|}
\hline$B$-Decay type & $\begin{array}{l}\text { Fraction of } \\
\text { total width }\end{array}$ & Description \\
\hline $\begin{array}{l}\text { Semileptonic } \\
\text { decays } \\
\text { decan }\end{array}$ & $25.0 \%$ & $\begin{array}{l}\text { The ISGW[37] form factor model is used } \\
\text { with modes for } D^{* *} \text { production added. The } \\
\text { fractions of decays producing charmed spec- } \\
\text { tator mesons are } 33 \%(D), 58 \%\left(D^{*}\right), \text { and } \\
9 \%\left(D^{* *}\right) . \text { The semileptonic branching frac- } \\
\text { tions are } 11.0 \%\left(e^{ \pm}\right), 11.0 \%\left(\mu^{ \pm}\right) \text {and } 3.0 \%\left(\tau^{ \pm}\right)\end{array}$ \\
\hline $\begin{array}{l}\text { Two } \\
\text { body hadronic } \\
\text { decays }\end{array}$ & $12.5 \%$ & $\begin{array}{l}\text { Exclusive branching ratios based on various } \\
\text { measurements of two body hadronic decays } \\
\text { according to the PDG[38]. }\end{array}$ \\
\hline $\begin{array}{l}B \text { mesons with } \\
\text { charm baryons }\end{array}$ & $6.0 \%$ & $\begin{array}{l}\text { Produced by means of internal } W \text {-emission } \\
\text { diagrams with diquark popping in the frag- } \\
\text { mentation involving the charm quark. }\end{array}$ \\
\hline Others & $56.5 \%$ & \begin{tabular}{l} 
decayed by LUND \\
\hline
\end{tabular}
\end{tabular}

Table 6.1: Distribution of the $B$ decay branching fraction.

The mean decay lifetimes of $B$-hadrons in the Monte Carlo are set to $1.55 \mathrm{ps}$ for $B$ mesons and $1.10 \mathrm{ps}$ for $B$ baryons, which are in good agreement with current measurements as shown in Fig.s 6.3 and 6.4 [14]. The $B$ baryon production in the Monte Carlo amounts to $8.9 \%$ of $B$ hadron production which in turn translates to an average Monte Carlo $B$ hadron lifetime of 1.51 ps.

\subsection{Simulation of Charm Hadron Decays}

The charm simulation has also been adjusted to produce more accurate spectra. To obtain better agreement in the inclusive production rates of leptons, kaons[38] and the $D$-meson decay charged multiplicity distributions, decay modes for which there was a large uncertainty on their branching fractions were adjusted. Details on the simulation of the various decay modes are given in Table 6.2. The charmed hadron lifetimes are according to the PDG values shown in Table 6.3[38]. 


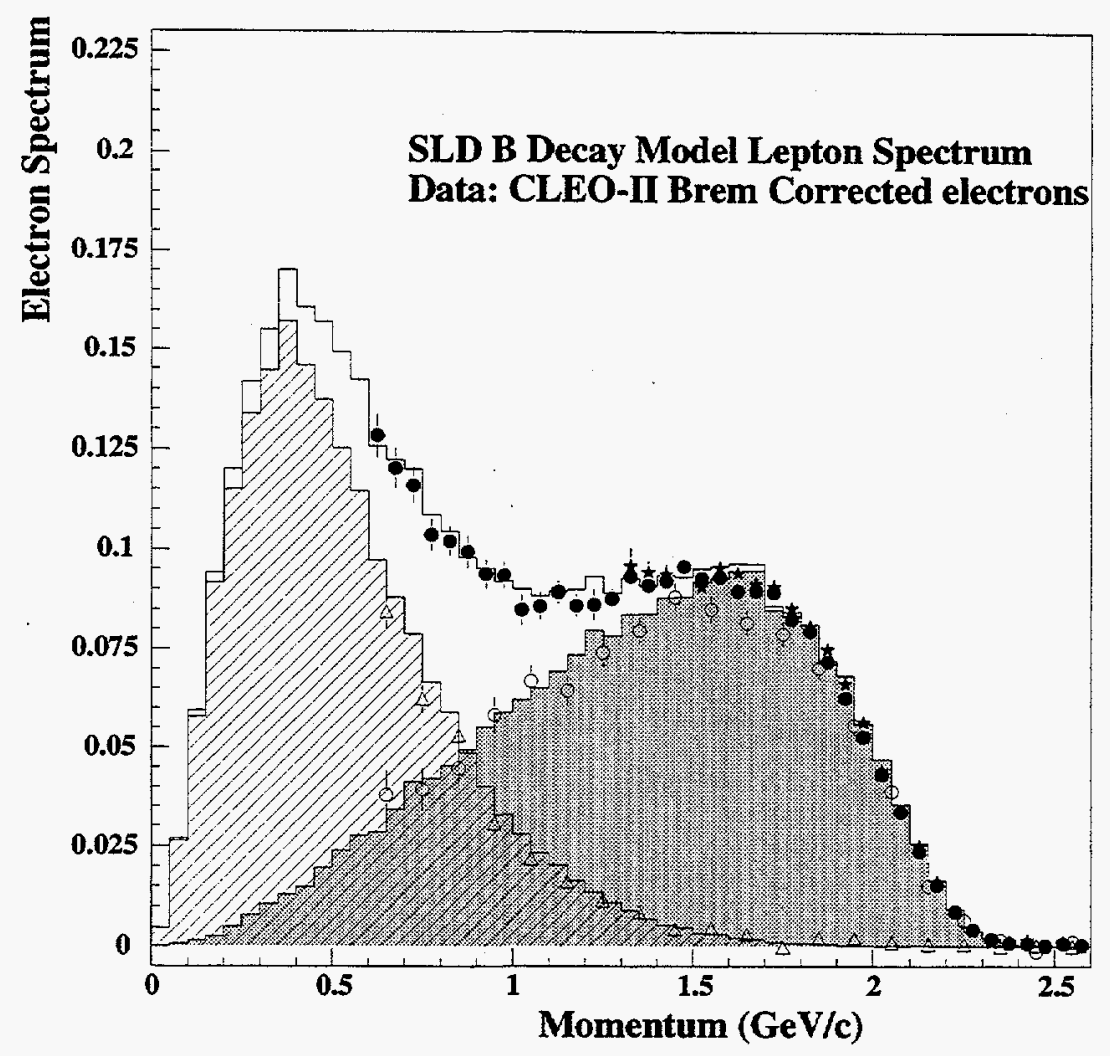

Figure 6.1: Spectrum of lepton momentum from $B$ decays compared between the Monte Carlo simulation and CLEO data.[34]

\subsection{QCD Simulation Parameters}

The thrust, sphericity, jet rates, particle multiplicity, and particle momentum distributions are functions of the fragmentation model. Within the model the control parameters include the minimum energy for a parton, leading logarithm scale which controls the evolution of the parton showers, variables controlling the fragmentation, and the width of the transverse momentum distribution for primary quarks. It has been shown by OPAL that the parameters determined by TASSO at $\sqrt{s}=35 \mathrm{GeV}$ [43] for QCD parton showers and LUND string fragmentation are consistent with data at the $Z^{0}$ energy[44]. The Monte Carlo corresponding to the 1992 and 1993 data was 

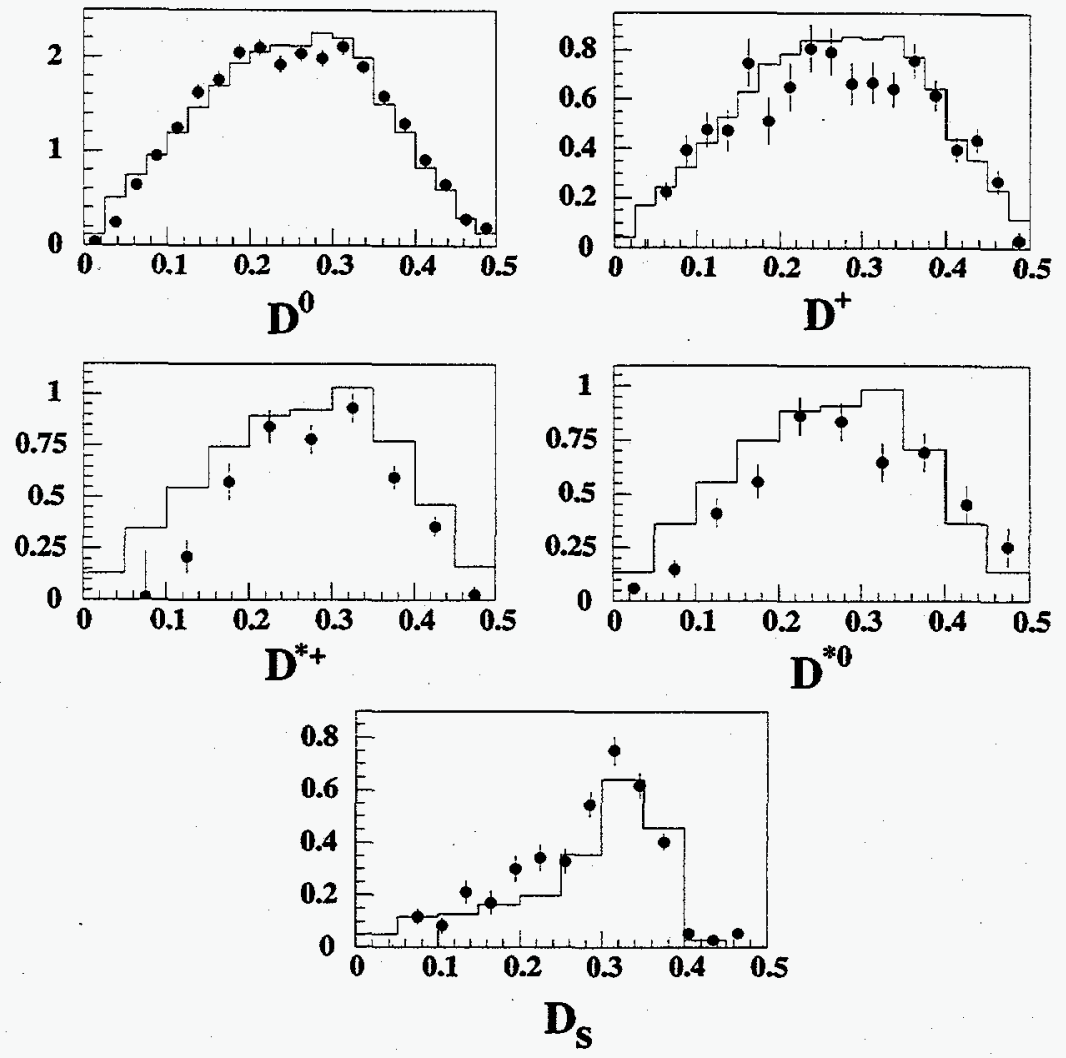

Figure 6.2: Spectra of different species of $D$ meson produced in $B$ hadron decays.[34]

generated using the TASSO parameters. Minor updates to the parameters were made for the 1994 Monte Carlo.

\subsubsection{Fragmentation}

Fragmentation models describe how hadrons are formed from the initial quarks[10]. In particular fragmentation functions are used which give the probability that a hadron will be formed at a given fraction of the quark energy carried by the hadron. Several models exists for the fragmentation functions with the most widely used one being the Peterson Function[45] shown in Eqn. 6.1. 


\section{$\tau_{b}$ Meson Measurements}

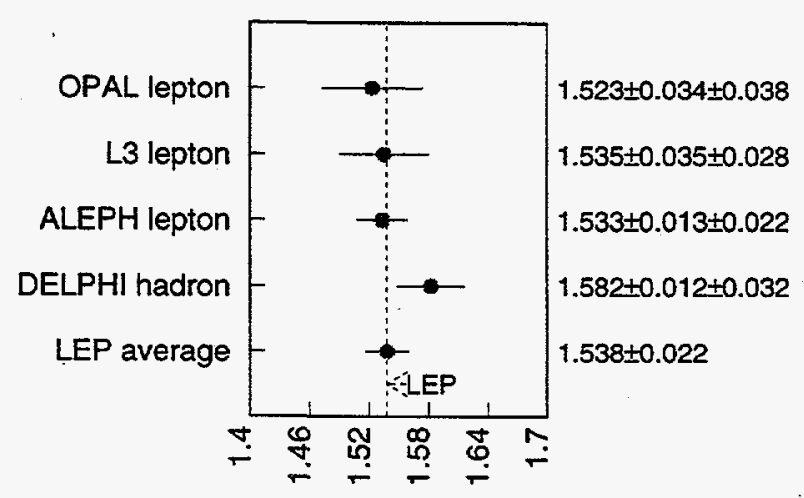

Figure 6.3: Recent $B$ meson lifetime measurements from LEP(Moriond 1995).

\section{$\tau_{\mathrm{b}}$ Baryon Measurements}

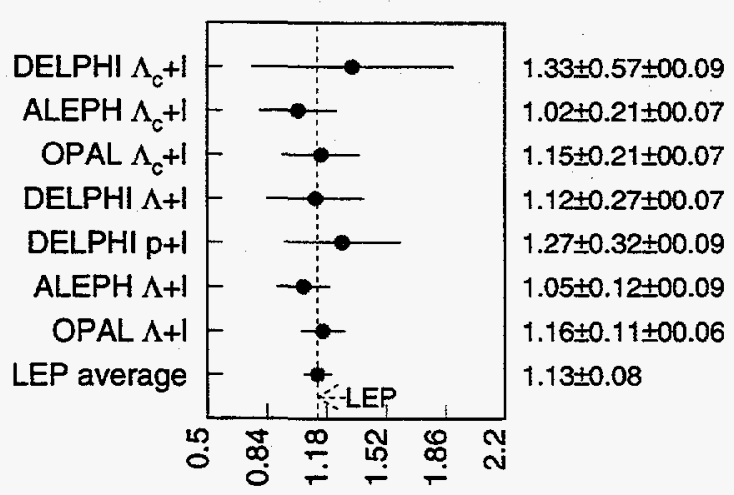

Figure 6.4: Recent $B$ baryon lifetime measurements from LEP(Moriond 1995).

$$
d \sigma / d z \sim z(1-z)^{2} /\left[(1-z)^{2}+\epsilon z\right]^{2} \quad, \quad z=\frac{\left(E+p\|\|_{\text {hadron }}\right.}{(E+p)_{\text {quark }}}
$$

The parameter $\epsilon$ controls the average energy of the hadron. The plot above shows the distributions for where the mean energy is set to that for $B$-hadrons and for $D^{*}$ 's. In our simulation values of $\epsilon_{b}=0.006$ for $B$ hadrons and $\epsilon_{c}=0.06$ for $D^{*}$ are 


\begin{tabular}{|l|l|}
\hline $\mathrm{D}$ decay type & Description \\
\hline $\mathbf{D}^{0}, \mathbf{D}^{+}, \mathbf{D}_{s}$ mesons $+\Lambda_{c}$ baryons & $\begin{array}{l}\text { branching ratios according to parti- } \\
\text { cle data group review[38] }\end{array}$ \\
\hline $\mathbf{D}^{*+}$ and $\mathbf{D}^{* 0}$ decay branching ratios & $\begin{array}{l}\text { updated to the recent measurements } \\
\text { from CLEO[41]. }\end{array}$ \\
\hline Meson 3-body semileptonic decay & WSB[42] form factor model \\
\hline $\begin{array}{l}\text { Weakly decaying charm baryons } \\
\text { excluding } \Lambda_{c}\end{array}$ & JETSET[32] \\
\hline Remaining decay modes & simulated by phase space distribution \\
\hline
\end{tabular}

Table 6.2: Summary of the charm decay model.

\begin{tabular}{|l|c|}
\hline Charmed Hadron & lifetime $(p s)$ \\
\hline$D^{+}$ & $1.057 \pm 0.015$ \\
$D^{0}$ & $0.415 \pm 0.004$ \\
$D_{s}$ & $0.467 \pm 0.017$ \\
\hline
\end{tabular}

Table 6.3: Charmed Meson Lifetimes from 1995 PDG

used. These correspond to average energies of $\left\langle x_{E}\right\rangle=0.695$ for $B$ hadrons in $b \bar{b}$ events and $\left\langle x_{E}\right\rangle=0.501$ for $D^{*}$ mesons in $c \bar{c}$ events and are in good agreement with the existing measurements[46]. Other models such as the Bowler Fragmentation model yield momentum distributions which are in reasonable agreement with current data but have a significantly different shape. For this reason, the variation in $R_{b}$ resulting from using different models will be included as a systematic.

The average charged particle multiplicity per event in the Monte Carlo is $\left\langle n_{c h}\right\rangle=21.1$ which is in agreement with current experimental measurements[47]. 


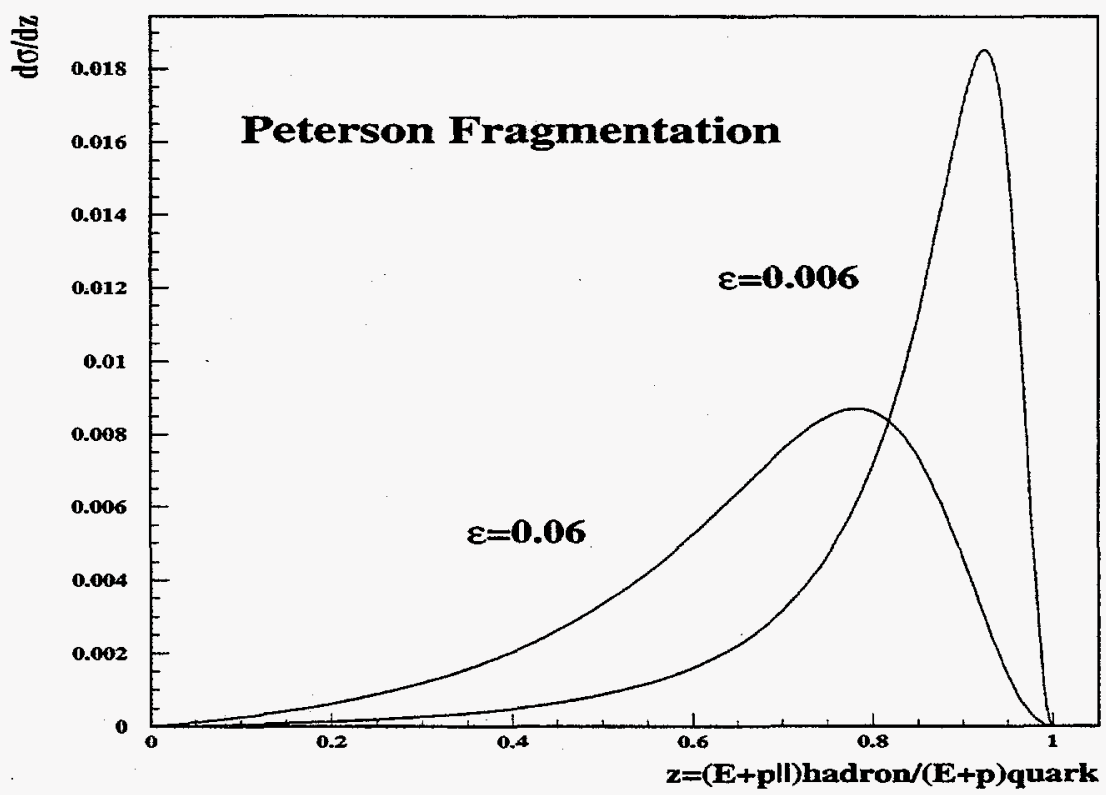

Figure 6.5: Peterson fragmentation distribution for $b$ and $c$ quarks. 


\section{Chapter 7}

\section{Event and Track Selection}

The events and tracks used by the tags must be purified of many possible backgrounds. For the events, potential contaminants from leptonic decays of the $Z^{0}$ and triggers resulting from machine backgrounds must be removed. While, for the tracks, those not resulting from the decay of the $Z^{0}$ and those tracks which are poorly measured must be removed. The event and track selection cuts common to both methods are described in this chapter. In addition, the correction procedure for discrepancies observed in the tracking distributions after event and track selection cuts have been applied will be presented.

\subsection{Hadronic Event Selection}

Most of the $e^{+} e^{-}$beam crossings at the SLD do not produce $Z^{0}$ decays. The crossings occur at a rate of $120 \mathrm{~Hz}$ but typically $Z^{0} \mathrm{~s}$ are produced at a rate of $0.8 \times 10^{-2} \mathrm{~Hz}$ to $1.6 \times 10^{-2} \mathrm{~Hz}$. To filter out the useful events, a particular event trigger and event selection scheme, as described below, is used.

\subsubsection{Event Triggers}

The event trigger is run during acquisition of data from the detector to eliminate events which clearly have no analysis use. Several triggers are used during data 
acquisition. Of these, one trigger, the hadron trigger plus EIT filter, selects events that are used by the $b$-tags. It is $93 \%$ efficient for passing hadronic decays. The trigger uses hardware tracking and calorimeter cuts as described below.

\section{- Calorimeter Cuts}

The algorithm requires at least $15 \mathrm{GeV}$ of energy in LAC towers passing a high threshold cut (240 MeV for EM towers, $1.2 \mathrm{GeV}$ for HAD towers). At least 10 towers must have contributed to the energy sums with at least one being in the forward and one being in the backward section of the barrel. The latter is to reduce triggers from single beam backgrounds which typically cause many hits on one end of the SLD only.

\section{- Track Cut}

Additionally, a track must have been found in the CDC. A track is identified, in the acquisition system, by constructing a map of cells which have hits on at least four wires. Then the list of these cells versus superlayer is compared against a lookup table for combinations which are consistent with nearly straight tracks. Any matching combination indicates a track. To avoid triggering on beam background bursts the trigger is vetoed if the number of CDC cells with at least six of the eight wires hit is $>275$.

\subsubsection{Event Selection}

Further event selection requirements are necessary to eliminate events outside the acceptance of the tracking systems, to reject leptonic decays and to ensure stable detector conditions. The following cuts have been chosen with these objectives in mind:

\section{- Acceptance Cut}

The thrust axis, reconstructed from refined calorimeter clusters, is required to lie well within the VXD acceptance $(|\cos \theta|<0.71)$. For this cut the coverage of the $\mathrm{LAC}$ is very important. Since no endcap tracking is currently used, any attempt 

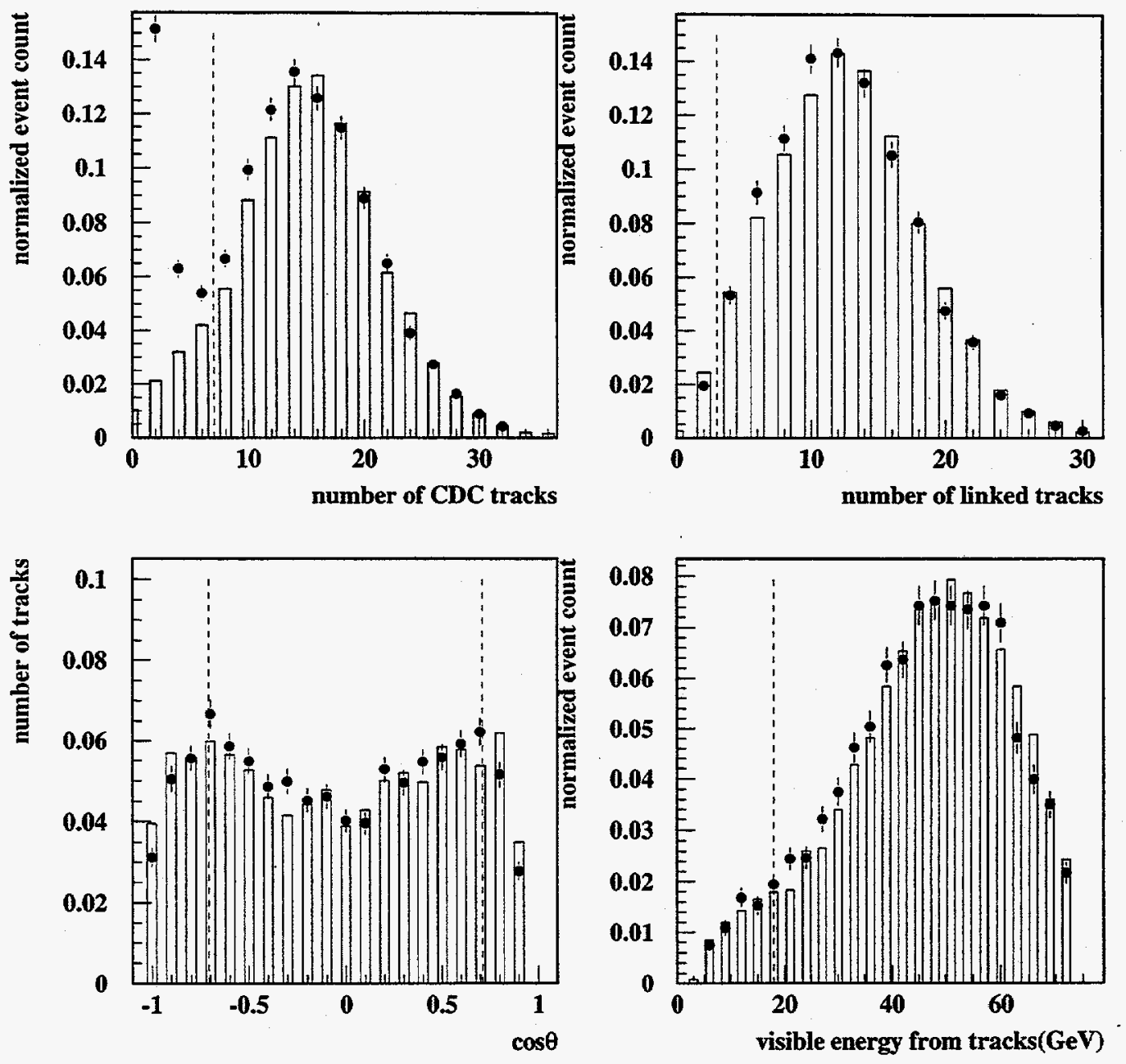

Figure 7.1: Distributions to which cuts are applied to select a pure hadronic event sample. The Monte Carlo is the line and data are the markers.

at using a thrust axis determined from tracks would cause significant biases near the edges of the CDC acceptance. As shown in Fig. 7.1, the acceptance of the CDC drops off rapidly at $|\cos \theta|=0.85$. The majority of hadronic events lost in the event selection are due to this cut. Approximately $61 \%$ of hadronic events pass. The cuts on the distributions are shown by the dashed lines in the figure. 


\section{- CDC Track Multiplicity and Visible Energy Cuts}

To eliminate leptonic $Z^{0}$ decays at least seven charged tracks must have been identified in the event. The distributions of the number of charged tracks in the Monte Carlo, which is of purely hadronic decays, and the data are shown in Fig. 7.1. Further purification of the sample is achieved by requiring that the amount of energy in the charged tracks be greater than $18 \mathrm{GeV}$. After these cuts are applied the contamination for leptonic events primarily from $\tau^{+} \tau^{-}$is found to be $<0.2 \%$. The cut on the multiplicity is shown by the dashed line in the figure.

\section{- VXD+CDC Track Multiplicity Cut}

For the 1992 data only, it was observed that occasionally an event passing the CDC track multiplicity cut would have no links to the VXD in the data. As is shown in Fig. 7.1, this does not occur in the Monte Carlo and the cause was not identified. To correct for this, events which have fewer that three linked $\mathrm{CDC}+\mathrm{VXD}$ tracks are eliminated. The cut is shown by the dashed line in the figure.

\section{- Known Bad Detector Operation Periods Removed}

Periods where documented VXD or CDC failures occurred have been eliminated. A period of the SLD 1993 run used an incorrect cell count cut for the CDC track trigger veto. This period has been eliminated from the data sample. No such periods exist for the LAC. As a further operational check on the tracking systems at least three tracks with two or more VXD links must exist in the event.

The count of events passing selection cuts are $37 \mathrm{k}$ for $1994,16 \mathrm{k}$ for 1993 , and $5 \mathrm{k}$ for 1992. The largest loss of events come from the acceptance cuts, removal of the 1993 bad veto period, and the unavailability of data collected in 1995 for the SLD 1994 run. The flavor bias introduced by this selection for $b$-quarks relative to all hadronic $Z^{0}$ decays is found from Monte Carlo to be less than $0.2 \%$. 


\subsection{Track Selection}

Not all reconstructed tracks are useful in the analysis. Tracks that can reduce the tagging performance of the tag are:

- tracks from single beam interaction with the beam-pipe or detector material near the beam-line

- CDC tracks that fail to link to a VXD hit and will thus have poor impact parameter resolution

- tracks suffering from many missing CDC hits

- low momentum tracks which can suffer from large multiple scattering

- kinked tracks that have scattered off of detector material

Tracks are selected in two steps. First, cuts are applied to the CDC track variables only, then cuts are applied to the combined CDC+VXD track. Tracks surviving all cuts are called quality tracks.

The CDC track cuts are:

\section{- Radius of innermost CDC measurement}

To insure that the track did not have to be extrapolated over a long arc the innermost measurement from the CDC must be within $39.0 \mathrm{~cm}$ of the beam line. As the extrapolation distance increases, the uncertainty on the track parameter also increases and becomes more likely to mislink to VXD hits.

\section{- Minimum Number of Hits Requirement}

Tracks with few hits are likely segments of a kinked track, a track that curved back towards the beam-line before traversing the full radius of the $\mathrm{CDC}$, a track from the decay of a very long lived neutral particle or a track that had hits stolen by another track. To eliminate these, the tracks are required to have at least 40 CDC hits. 


\section{- Impact Parameter Cuts}

To remove tracks from interactions with detector material a constraint is placed on the apparent origin of the track. This is done by requiring that the CDC track extrapolate within $1.0 \mathrm{~cm}$ in the $x y$ plane and $1.5 \mathrm{~cm}$ in the $r z$ plane.

\section{- Fit Quality Cut}

The quality of the fit of a helix to the CDC drift distance measurements is determined from the residual distances between the hits and the helix and the uncertainties from the drift model. A cut of $\frac{x^{2}}{d f}<5$ is used.

\section{- $V^{0}$ rejection}

Tracks from long lived neutral particle $\left(V^{0}\right.$ 's) decays are rejected using the procedure described in Sect. A.

The additional cuts on the CDC+VXD track are:

\section{- Impact Parameter Uncertainty Cut}

The 2-D impact parameter error from the track extrapolation alone is required to be $<250 \mu \mathrm{m}$. This eliminates both poorly measured tracks and acts as a momentum cut of $\sim 300 \mathrm{MeV}$.

\section{- Impact Parameter Cut}

A cut of $3 \mathrm{~mm}$ is used to help remove very long lived particle decays that were not rejected by the $V^{0}$ removal procedure.

\section{- Fit Quality Cut}

To reduce the possibility of having an incorrect vertex detector hit linked to the $\mathrm{CDC}$ track the quality of the CDC+VXD fit is required to satisfy $\frac{x^{2}}{d f}<5$.

The fraction of CDC tracks passing each of the CDC quality cuts is compared between data and Monte Carlo to check for any cut on a poorly simulated distribution. No significant discrepancies are seen. A similar check is performed for the fraction of good CDC tracks that link in the data and Monte Carlo. Both comparisons are shown in Fig. 7.2. 

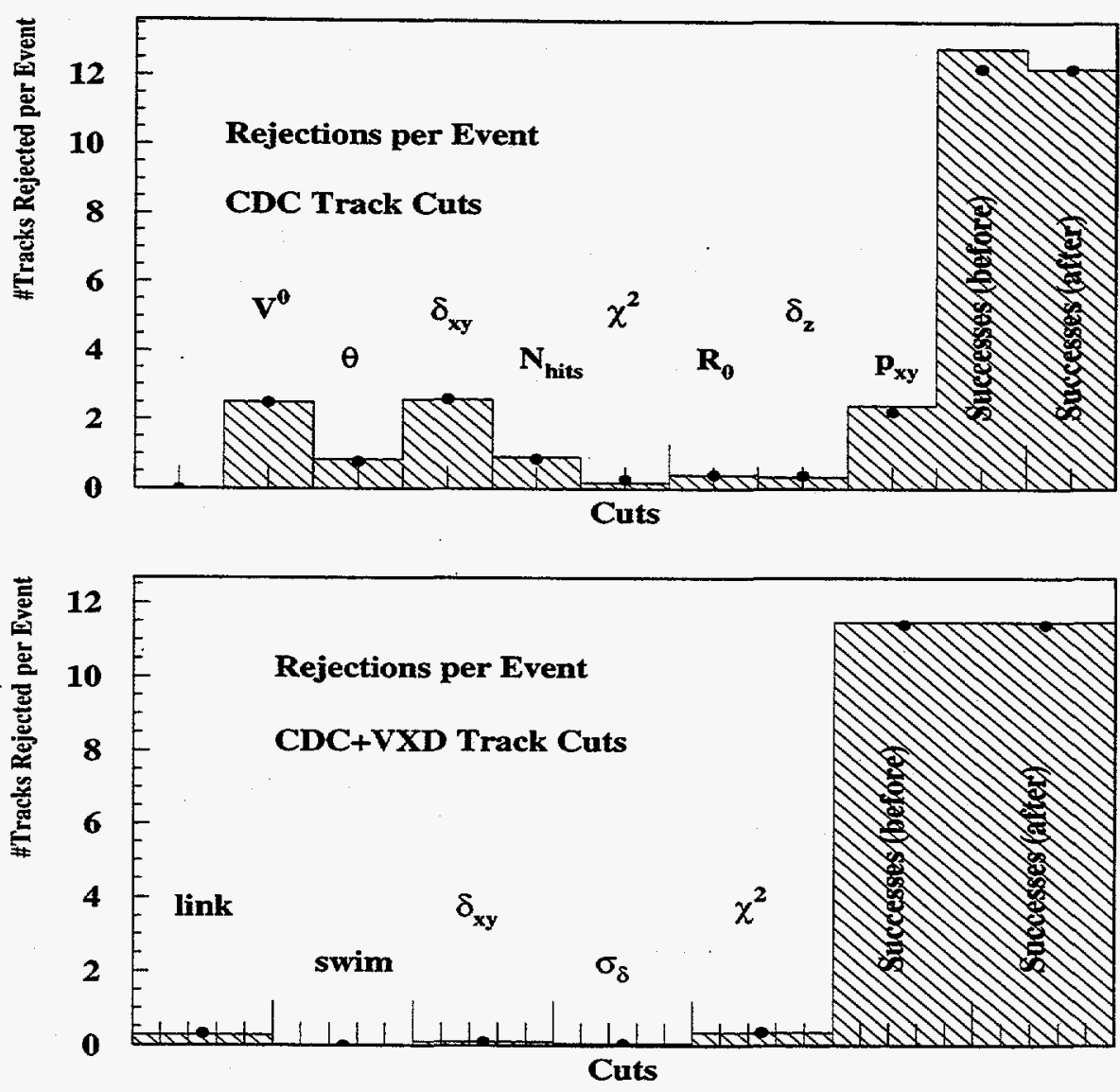

Figure 7.2: Number of tracks per event failing each track selection cuts for the CDC track alone and the CDC+VXD track. The last two bins show the number of tracks that pass the selection before and after efficiency corrections have been applied.

\subsection{Tracking Efficiency Corrections}

The fraction of tracks passing the quality cuts is different between data and Monte Carlo. The exact cause is not known. The average multiplicity of quality tracks in the Monte Carlo is 12.81 and for the data it is 12.24 . This is $\sim 2 \sigma$ of the $Z^{0}$ decay multiplicity uncertainty. An example of a simulation problem that caused a $\sim 1 \%$ discrepancy was a simplified simulation of the dependence of the hit efficiency on the position within a CDC cell. An example of the hit efficiency dependence on the drift distance is shown in Fig. 7.3. The drop in efficiency occurs near the field shaping wires where the drift velocity is not well known and was previously not well simulated. 
However, after correcting this discrepancy the $\approx 4.7 \%$ discrepancy mentioned above remained.

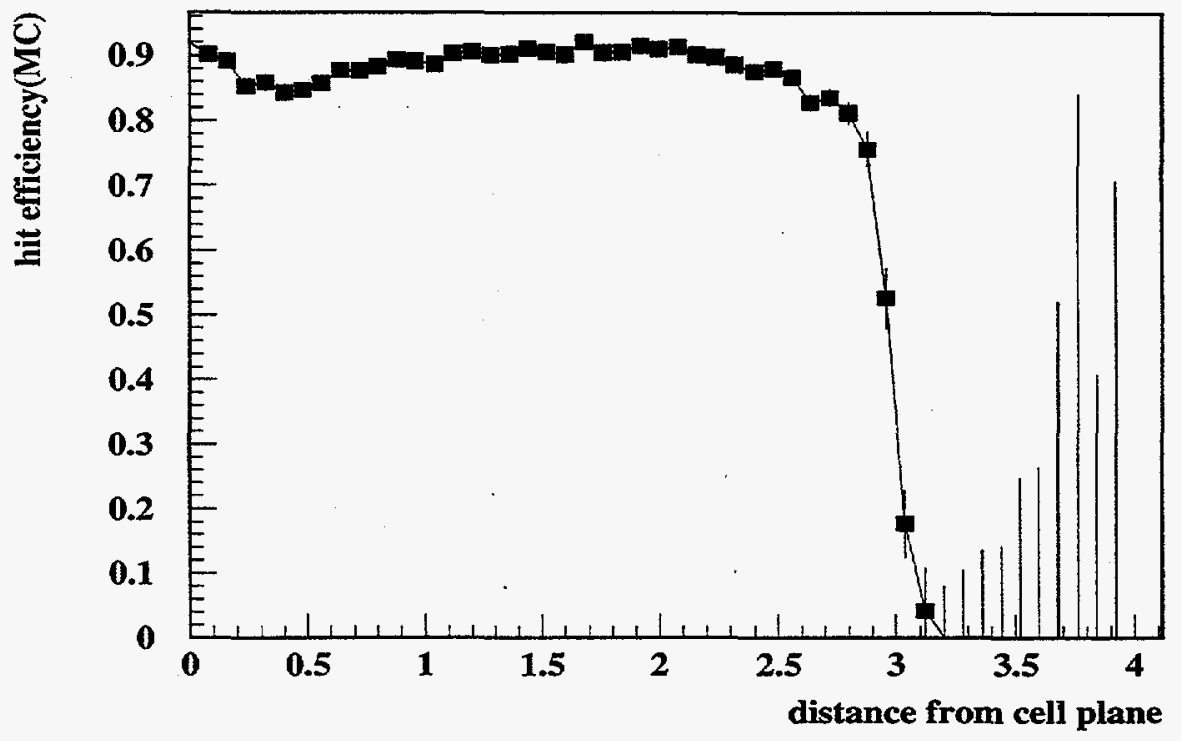

Figure 7.3: Hit efficiency versus drift distance in the CDC.

To correct for observed differences in the multiplicity of good tracks between data and the Monte Carlo, position and momentum dependent efficiency corrections are introduced in the simulation. The corrections are applied to the multiplicity of tracks passing the CDC cuts and the fraction of those tracks that link, separately. The . number of tracks per event passing the CDC quality cuts are binned in:

- $\phi$, to account for problems with CDC motherboards or cells

- $\cos \theta$ to account for problems with pulse height simulation and and unsimulated inefficiencies near wire ends

- momentum transverse to the beam line

- angle between the track and the jet it belongs to. A difference in the rate of tracks lost in the core of jets can result from improperly simulated resolutions 
The distribution of the efficiencies is shown in Fig. 7.4. To correct the simulation tracks are randomly removed with a frequency determined by which bin the tracks are associated with.
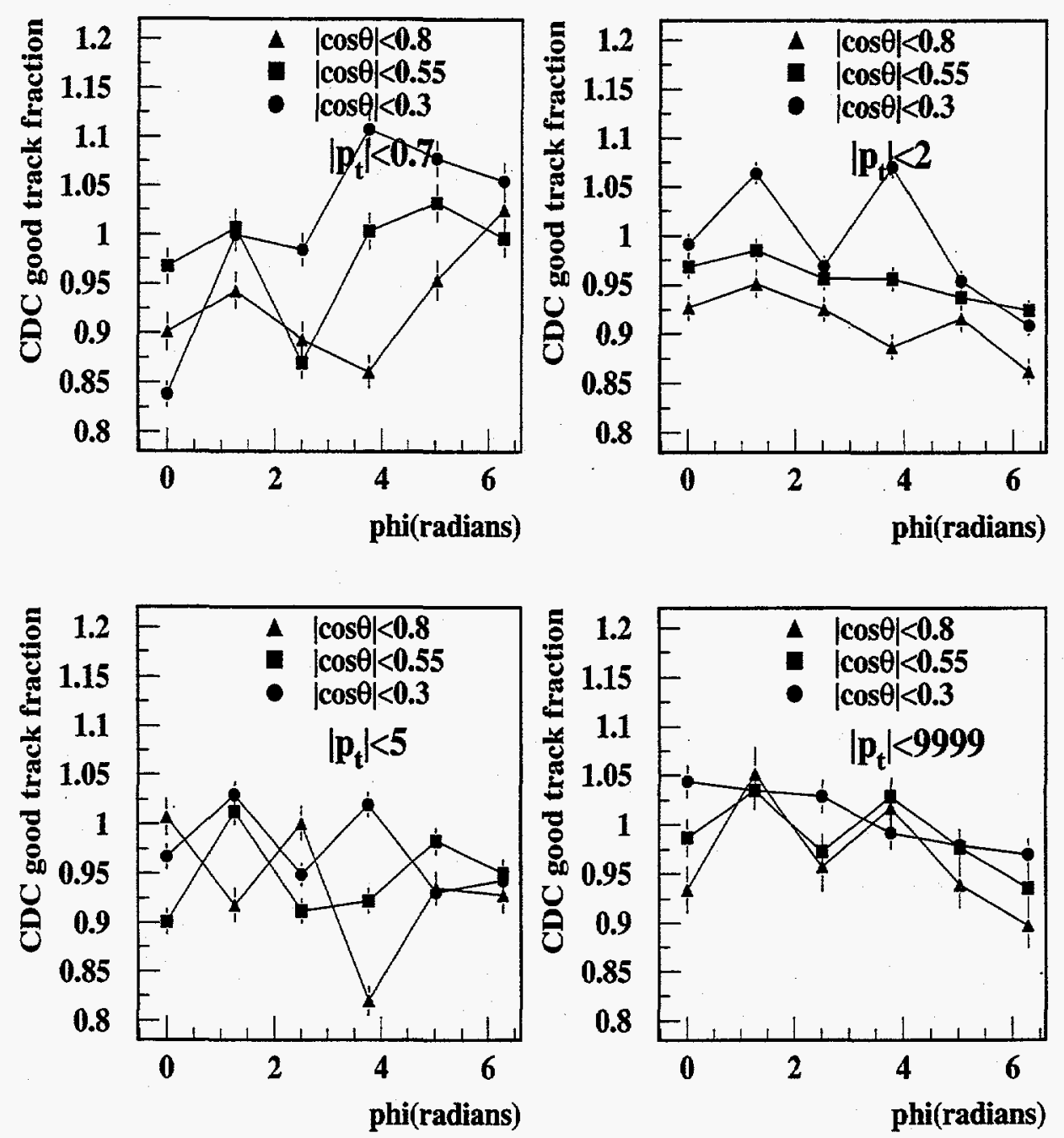

Figure 7.4: Efficiency corrections applied to the Monte Carlo for quality CDC tracks.

It is important to apply the efficiency corrections before the $V^{0}$ rejection is applied. This is due to the fact that if one of the tracks of a $V^{0}$ decay was lost to some inefficiency, the $V^{0}$ would never have been identified. Failure to perform the efficiency corrections before the $V^{0}$ rejection results in too many tracks being thrown out of the Monte Carlo. 
The fraction of good CDC tracks that linked to VXD hits was binned in:

- $\phi$, to account for inefficient ladders

- $\cos \theta$, to account for inefficient CCDs

- total momentum to account for inefficiencies due to pulse hight cuts on the VXD hits

The distribution of the efficiencies is show in Fig. 7.5.

An average of about $4.7 \%$ of the Monte Carlo tracks passing the CDC quality cuts are removed and a negligible amount of links are removed. Indications of a particular cut causing the difference in the fraction of tracks passing but such a problem would clearly show up in Fig. 7.2. Further indication that the cuts are equally affecting data and Monte Carlo tracks is in the fact that the initial discrepancy observed in the mean number of tracks before cuts is equal to that after cuts have been applied. This is shown in Fig. 3.20. After the corrections are applied the discrepancy is eliminated.

\subsection{Tracking Resolution Corrections}

Tracks that appear not to come from the primary vertex can be eliminated from the impact parameter distributions as described in Sect. 5.1.1. The resulting distribution is indicative of the convolution of the detector resolution and the primary vertex resolution. This distribution is called the negative impact parameter distribution and is shown in Fig. 7.6. The negative impact parameter distribution from the data and the Monte Carlo are compared in the $r \phi$ and $r z$ projections to determine the residual amount of resolution degradation to be applied to the Monte Carlo.

A procedure was developed to identify any deficiencies in the simulation of the distributions and to correct them. A fraction of the tracks have the resolution of the impact parameters degraded by a Gaussian of varying width. For the various fractions and widths the $\chi^{2}$ of the match between the distribution of the data impact parameters and the degraded Monte Carlo impact parameters was plotted. This was performed for three polar regions $(0.00>|\cos \theta|<0.3,0.30>|\cos \theta|<0.55,0.55>$ 

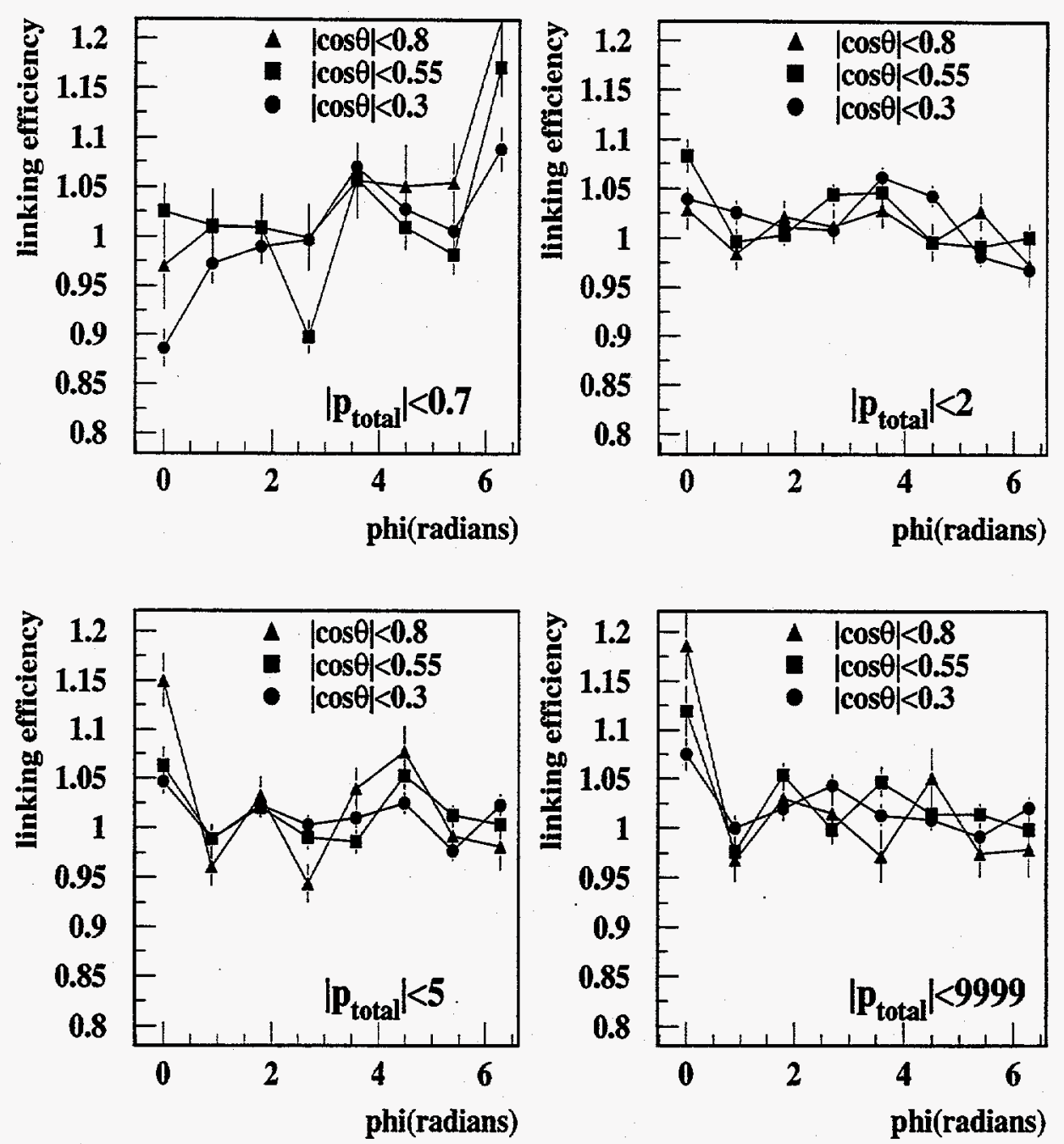

Figure 7.5: Efficiency corrections applied to the Monte Carlo for the rate that quality CDC tracks link to VXD hits.

$|\cos \theta|)$ that approximately corresponded to CCD boundaries, and four momentum regions $(0.0<p<0.7,0.7<p<2.0,2.0<p<5.0,5.0<p)$. For the mid $\cos \theta$ region the result is shown in Fig. 7.7. The contour lines correspond to values of $\chi^{2}=(1,2,3)$. In $r \phi$ the required width of the Gaussian used for degrading the resolution of the core of the distribution was determined to be less than $4 \mu \mathrm{m}$ and no degradation is applied. Also, no adjustment for the tail was found to be necessary. In $r z$ the width of the Gaussian degradation function needed to obtain the best match of the Monte Carlo 

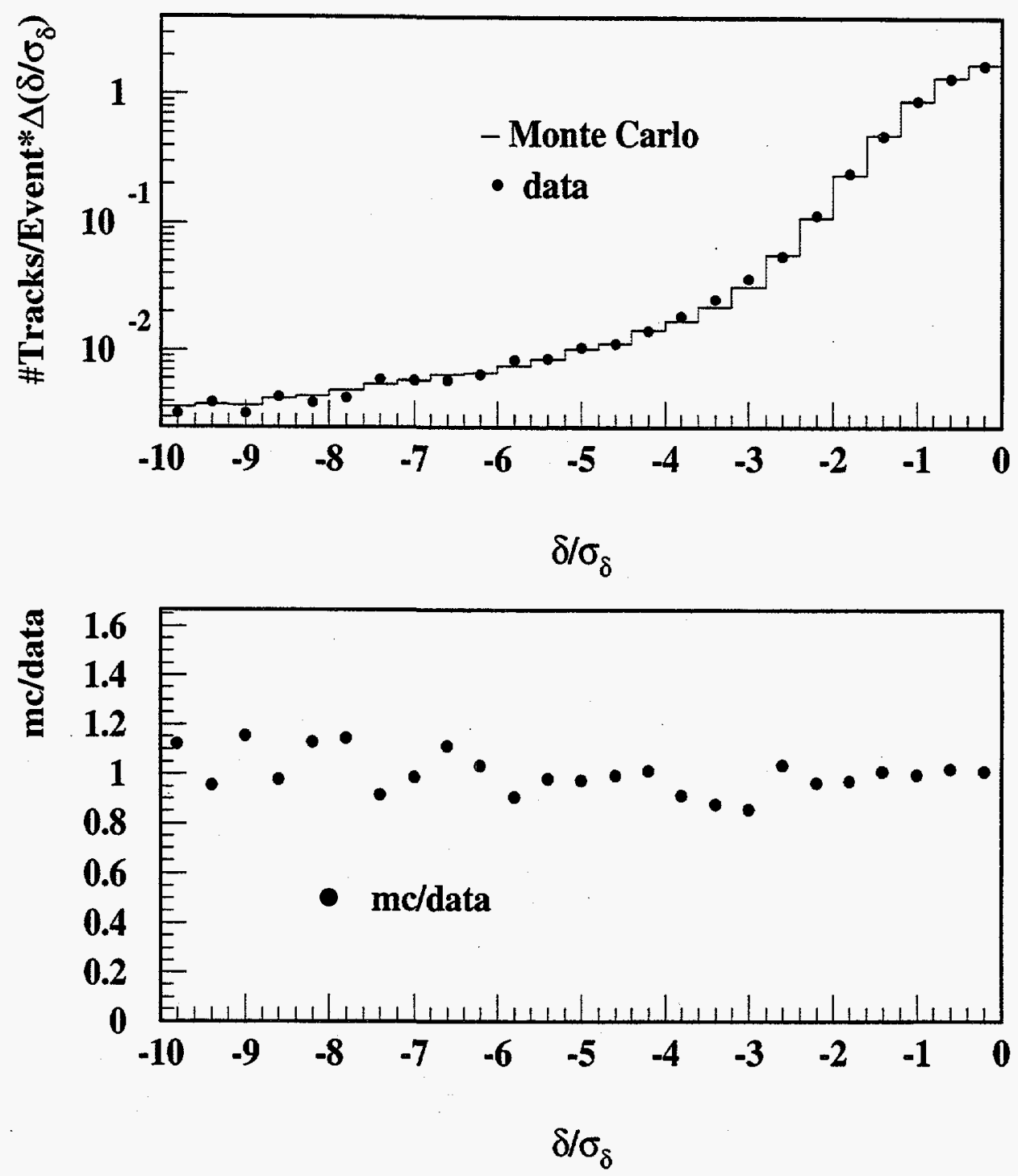

Figure 7.6: Distribution of the impact parameter divided by its uncertainty showing the accurate simulation of the resolution from the Monte Carlo.

core with the data was determined to be $\frac{16 \mu m}{\sin \theta}$ for tracks in 1992 and 1993 Monte Carlo and $\frac{24 \mu m}{\sin \theta}$ for 1994 Monte Carlo. No adjustment for the non-Gaussian tail was required. Systematic $\phi$ dependent shifts of the track $z$ at the $x y$ point of closest approach $\left(z_{\text {doca }}\right)$ with magnitudes typically around $\pm 20 \mu \mathrm{m}$ were observed in the data and are applied to the $z_{d o c a}$ of the Monte Carlo tracks. 

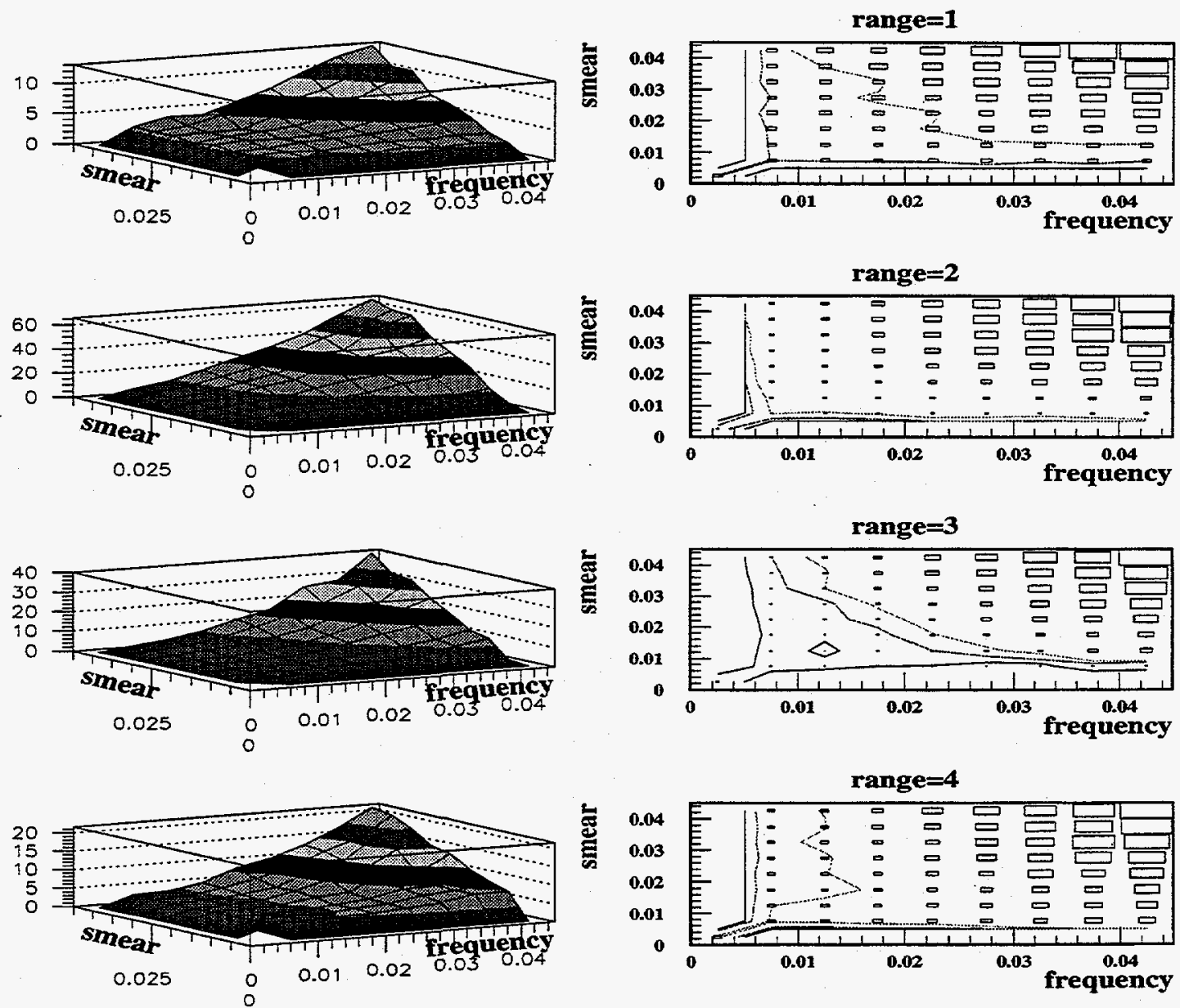

Figure 7.7: $\chi^{2}$ of the fit of the data and Monte Carlo impact distributions versus width of the Gaussian used to degrade the resolution in the Monte Carlo and the frequency with which it is applied. 


\section{Chapter 8}

\section{The 2-D Impact Parameter Event Tag}

\subsection{Introduction}

The previous chapters have detailed how the data is obtained and the events are selected and corrected. This chapter describes how $R_{b}$ is measured using the 2-D Impact Parameter Event Tag to select a pure sample of $Z^{0} \rightarrow b \bar{b}$ events. For this method, the $r \phi$ normalized impact parameter is used as the tag variable. The $b$ tagging power provided by this variable is shown in Fig. 8.1. Here one sees that $b \bar{b}$ events have a large excess of tracks with large positive $r \phi$ normalized impact parameters. It will be a cut on the number of such tracks that will be used to $b$-tag events. A method similar to this was first used by the MARK II experiment[48]. Here, the much larger dataset and more precise tracking of the SLD experiment are exploited to improve on the measurement.

\subsection{Procedure}

We start with events passing selection cuts from the 1992 and 1993 SLD runs. For various tag cuts the $b$-tagging efficiencies for $b, c$ and $u d s$ events are measured. These are then used to apply corrections for light quark contamination to the $b$-tagging 

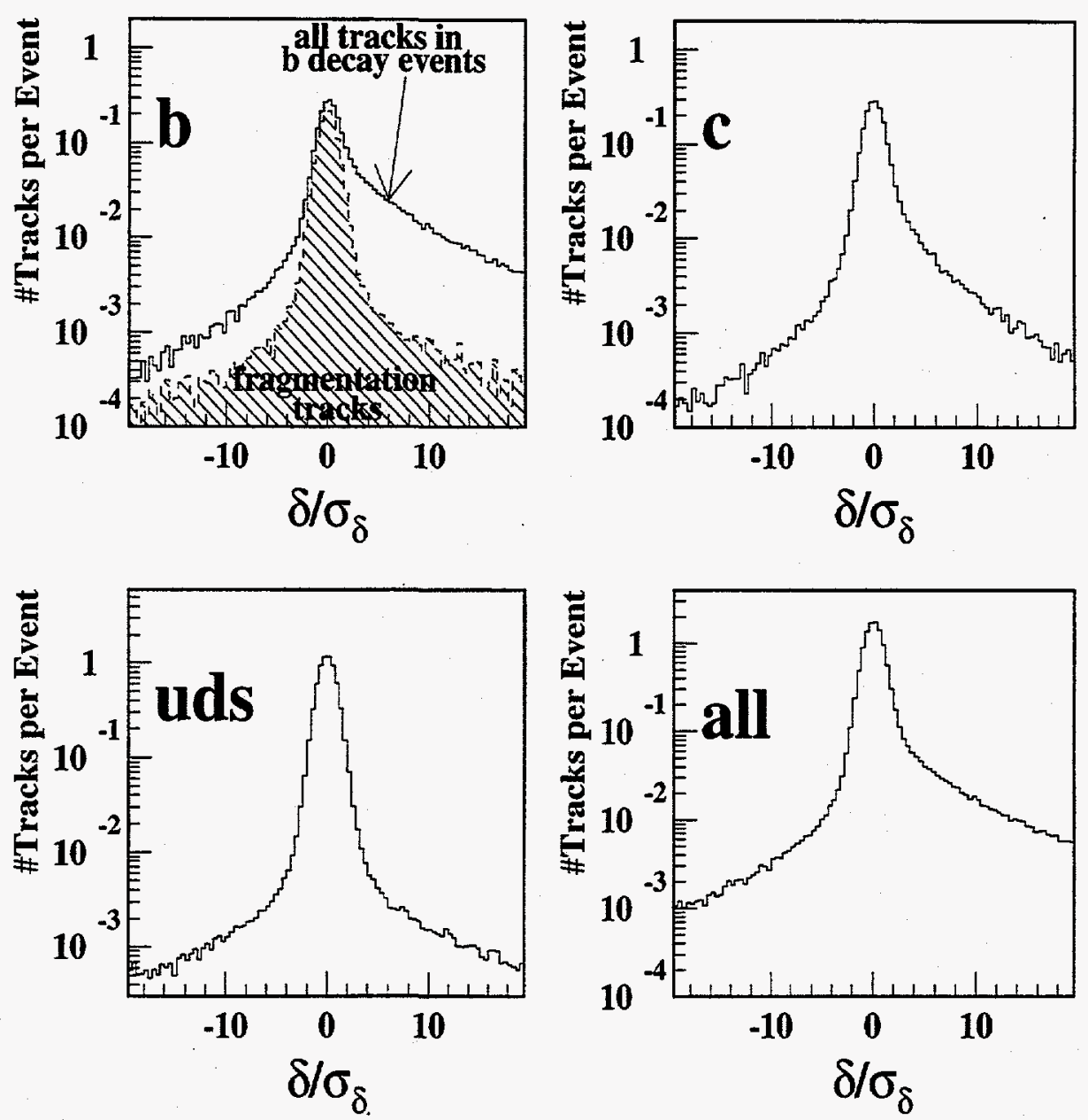

Figure 8.1: $r \phi$ normalized impact parameters for $b, c, u d s$, and all Monte Carlo and data events.

rates in data to measure $R_{b}$. Then, the systematics and statistical uncertainties are estimated for these cuts and the cuts which minimize the net uncertainty on $R_{b}$ are selected. First, we start with a discussion of the means by which $R_{b}$ is obtained from the $b$-tagging rates. 


\subsubsection{Determining $R_{b}$ from Event Tag Counts}

We start by determining the number of tagged events given the $b$-tagging efficiencies for $b \bar{b}\left(\epsilon_{b}\right), c \bar{c}\left(\epsilon_{c}\right)$ and $u \bar{u}+d \bar{d}+s \bar{s}\left(\epsilon_{u d s}\right)$ events. Biases introduced by the event selection are accounted for by correction factors $\left(\eta_{q}\right)$ for each of the corresponding efficiencies. The tag count is then given by Eqn. 8.1.

$$
n_{t a g}=\epsilon_{b} \eta_{b} n_{b}+\epsilon_{c} \eta_{c} n_{c}+\epsilon_{u d s} \eta_{u d s} n_{u d s}
$$

Dividing this by the total number of events passing selection cuts and using the definitions of the hadronic width ratios as shown in Eqn. 8.2, the fraction of events tagged $\left(f_{\text {tag }}\right)$ is given by Eqn. 8.4.

$$
\begin{gathered}
R_{b}=\frac{n_{b}}{n_{\text {hadronic }}}, R_{c}=\frac{n_{c}}{n_{\text {hadronic }}}, R_{u d s}=\frac{n_{u d s}}{n_{\text {hadronic }}} \\
f_{\text {tag }}=\frac{n_{\text {tag }}}{n_{\text {hadronic }}}=\epsilon_{b} \eta_{b} R_{b}+\epsilon_{c} \eta_{c} R_{c}+\epsilon_{u d s} \eta_{u d s} R_{u d s} \\
=\frac{n_{\text {tag }}}{n_{\text {hadronic }}}=\epsilon_{b} \eta_{b} R_{b}+\eta_{u d s c}\left(\epsilon_{c} R_{c}+\epsilon_{u d s}\left(1 .-R_{b}-R_{c}\right)\right)
\end{gathered}
$$

This is then solved for $R_{b}$ resulting in the expression of Eqn. 8.5.

$$
R_{b}=\frac{f_{t a g}-\epsilon_{u d s}-\left(\epsilon_{c}-\epsilon_{u d s}\right) R_{c}}{\epsilon_{b}-\epsilon_{u d s}+\xi_{b}\left(\epsilon_{b}-f_{t a g}\right)}
$$

In Eqn. 8.5 the fact that $\eta_{c}=\eta_{u d s}$ within the statistical uncertainty of 0.3 is used. The event selection bias correction $\xi_{b}$ is defined to be $\eta_{b} / \eta_{u d s c}-1$. The value of $R_{c}$ is set to 0.17 which is the Standard Model value. This is a standard practice as the experimental uncertainties in $R_{c}$ are large relative to the theoretical prediction. However, instead of using the theoretical uncertainty on this number the uncertainty on the world average of the $R_{c}$ measurements is used. Along with the tagging efficiencies the $b$-purity of the tagged events is used as monitor of the $b$-tag performance. The purity is defined by

$$
\Pi_{b}=\frac{\epsilon_{b} \cdot R_{b}^{S M}}{\epsilon_{b} \cdot R_{b}^{S M}+\epsilon_{c} \cdot R_{c}^{S M}+\epsilon_{u d s} \cdot R_{u d s}^{S M}}
$$

where $S M$ denotes that the Standard Model value is used. 


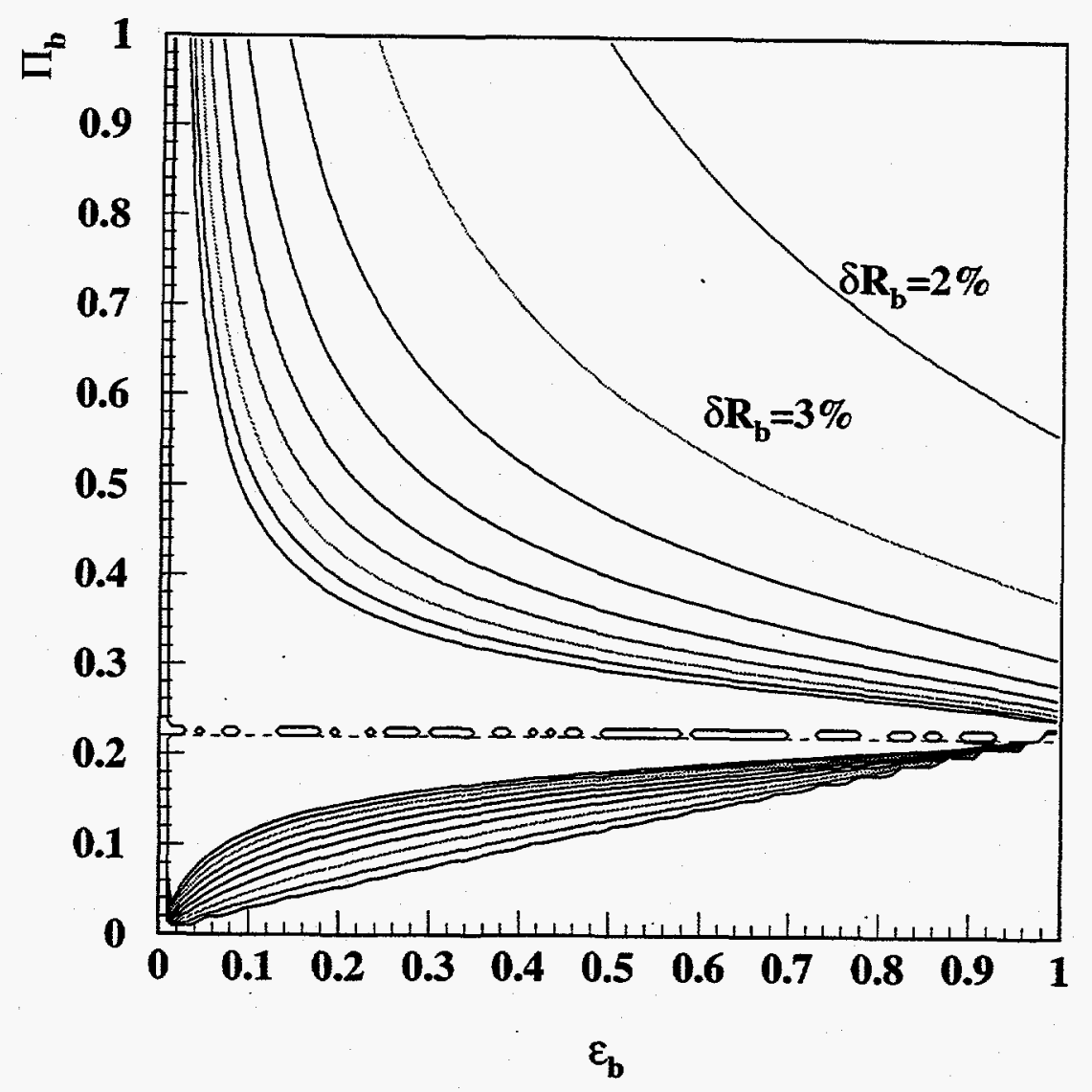

Figure 8.2: Statistical uncertainty on $R_{b}$ for a fiducial data sample of $21 \mathrm{k}$ events versus $\epsilon_{b}$ and $\Pi_{b}$. The curves for $\Pi_{b}<R_{b}$ are unphysical since they correspond to anti-tagging $b$ events. With no tag $\Pi_{b}=R_{b}$.

\subsubsection{Statistical Uncertainties}

The statistical uncertainties come from the errors on the counting rates. The most significant of these is the uncertainty on $f_{\text {tag }}$ but small non-negligible contributions come from the efficiencies. The contributions from each are added in quadrature as shown in Eqn. 8.7. Since the tag count is restricted by the total count, binomial statistics are used. The final form for the uncertainty in $R_{b}$ from the tagging rates is given in Eqn. 8.9. 

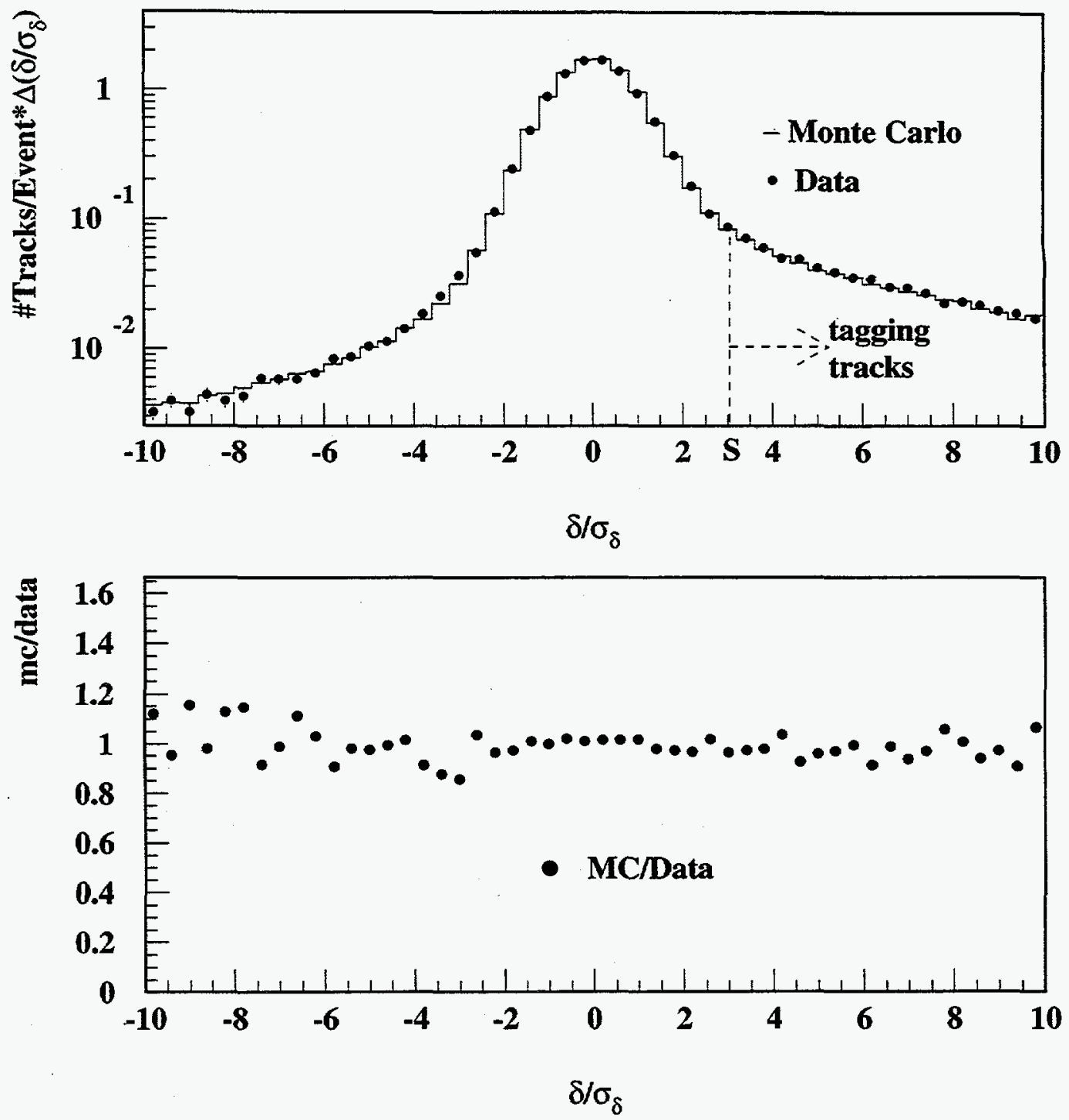

Figure 8.3: Normalized impact parameter distribution for Monte Carlo and data after resolution and efficiency corrections have been applied.

$$
\begin{gathered}
\left(\delta R_{b}\right)^{2}=\left(\frac{\delta R_{b}}{\delta f_{\text {tag }}}\right)^{2} \delta f_{\text {tag }}^{2}+\left(\frac{\delta R_{b}}{\delta \epsilon_{b}}\right)^{2} \delta \epsilon_{b}^{2}+\left(\frac{\delta R_{b}}{\delta \epsilon_{c}}\right)^{2} \delta \epsilon_{c}^{2}+\left(\frac{\delta R_{b}}{\delta \epsilon_{u d s}}\right)^{2} \delta \epsilon_{u d s}^{2} \\
\text { binomial statistics: } \delta_{f_{i}}^{2}=f_{i}\left(1-f_{i}\right) / N_{i}
\end{gathered}
$$




$$
\delta R_{b}^{2}=\frac{f_{\text {tag }}\left(1-f_{\text {tag }}\right)}{N_{\text {data }}}+R_{b}^{2} \frac{\epsilon_{b}\left(1-\epsilon_{b}\right)}{N_{b}}+R_{c}^{2} \frac{\epsilon_{c}\left(1-\epsilon_{c}\right)}{N_{c}}+\left(1-R_{b}-R_{c}\right)^{2} \frac{\epsilon_{u d s}\left(1-\epsilon_{u d s}\right)}{N_{u d s}}
$$

The statistical uncertainty on $R_{b}$ versus the $b$-tag efficiency and purity are shown in Fig. 8.2. Tags which result in both high $\epsilon_{b}$ and high $\Pi_{b}$ will have the lowest statistical uncertainty. As the efficiency decreases the count of events from which the result is derived dwindles causing higher statistical uncertainty on $\epsilon_{b}$. As the purity decreases the uncertainty from background contamination statistics increases.

\subsubsection{The Tag}

In the 2-D Impact Parameter Event Tag an event is $b$-tagged by selecting events with $N_{S}$ quality tracks having $r \phi$ normalized impact parameter $\delta_{\text {norm }}$ (described in Sect. 5.1.2) greater than a selected cut $S$. The $S$ cut is illustrated in Fig. 8.3 which shows the distribution of $r \phi$ normalized impact parameters in data and Monte Carlo. One sees from the ratio of the distributions that the data distribution is well simulated by the Monte Carlo.

\subsection{Measurement Results}

The values measured for $R_{b}$ for various $N_{S}$ and $S$ cuts are shown in Fig. 8.6. The optimal cut will be $S=3.0$ and $N_{S}=3$. This is based on the statistical and systematic uncertainties on $R_{b}$. It will be justified in Sect. 8.6 after the systematics have been presented. The Monte Carlo estimation of the tag performance at this cut is given in Table 8.2. In the 1993 data 2617 events of the 16235 events passing selection cuts are tagged and 815 of 5125 events in 1992 are tagged. These measurements were combined according to Eqn. 8.5 to obtain the results shown in Table 8.1, where the uncertainty is statistical only.

The curves of $b$-tagging efficiency versus purity of the tagged sample are shown in Fig. 8.4 for fixed multiplicity cuts while the significance cut is allowed to vary along the curve. At $S=3.0$ the multiplicity of tracks per event is as shown in Fig. 


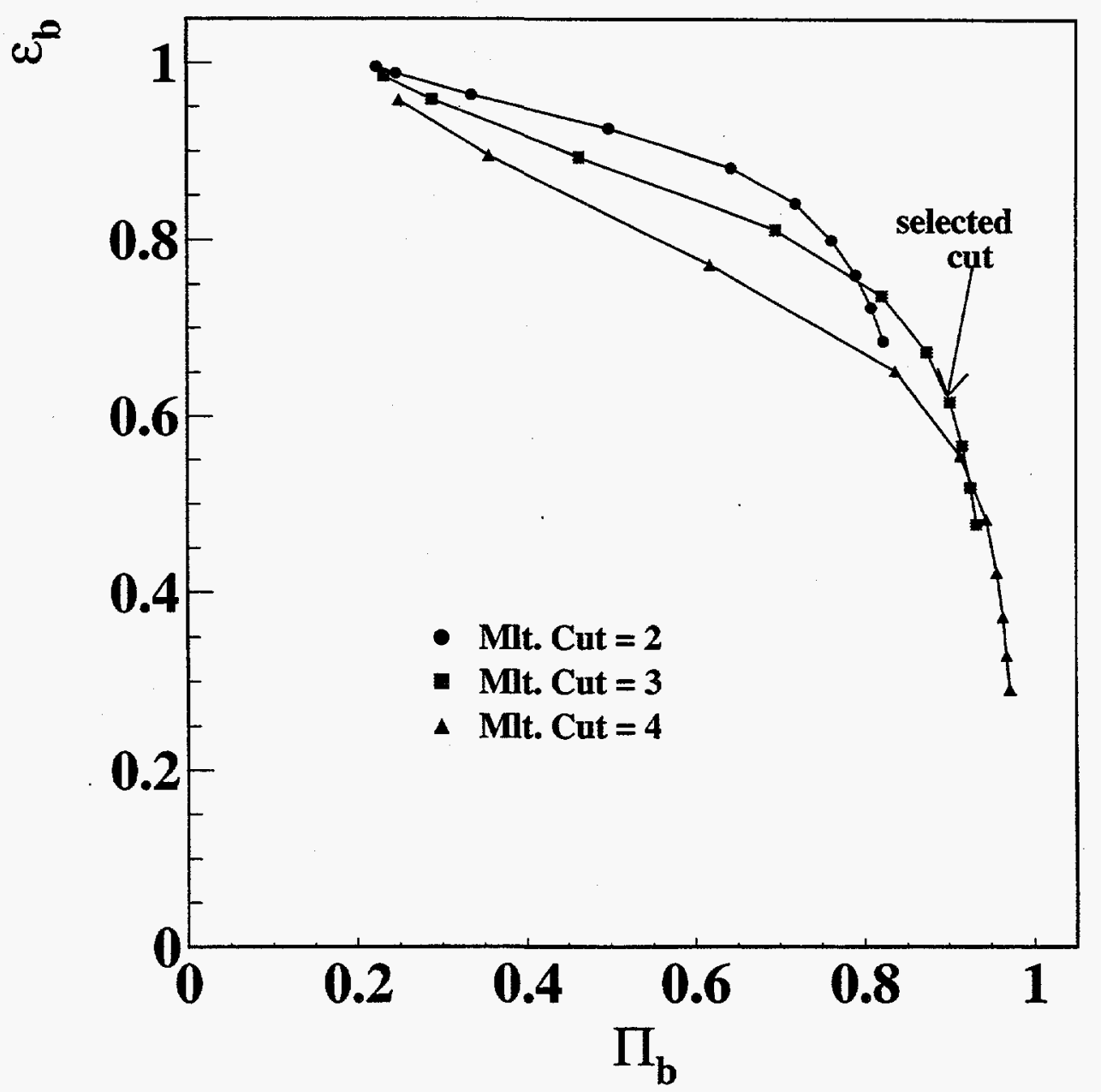

Figure 8.4: Plot of the tagging efficiency versus purity for $b$-quark events.

8.5. There is good agreement between the Monte Carlo total count and the data over the full range. All tagged events (i.e. those passing $N_{S}=3$ ) appear in the bins to the right of 3 on this plot. 


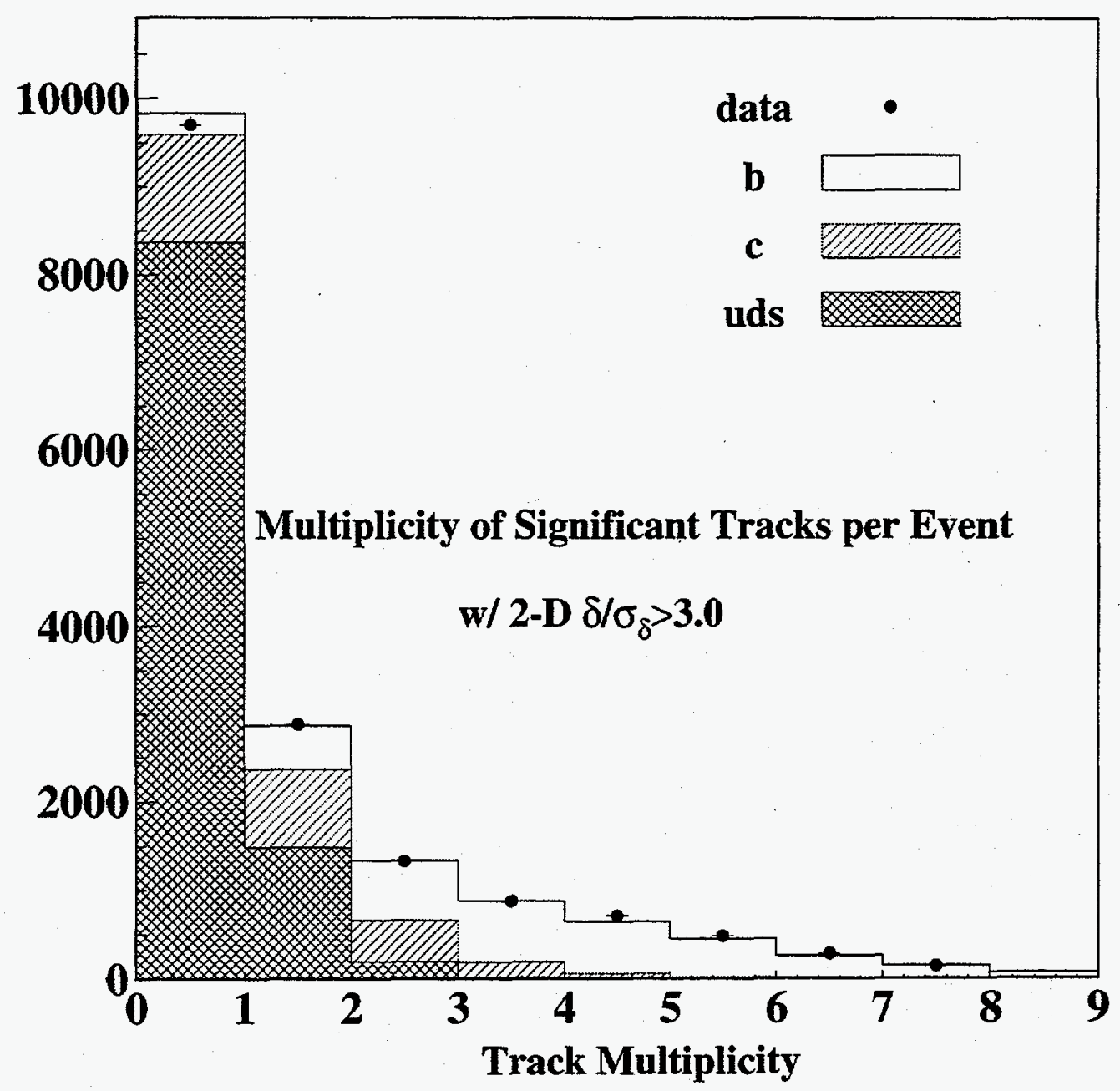

Figure 8.5: Plot of the number of tracks per event with significance $>3 \sigma$.

\subsection{Systematics}

Systematical uncertainties in $R_{b}$ come from the uncertainties in the physics and detector simulation used to estimate the $b$-tagging efficiencies. The sources of these systematics and their magnitudes are described below. 


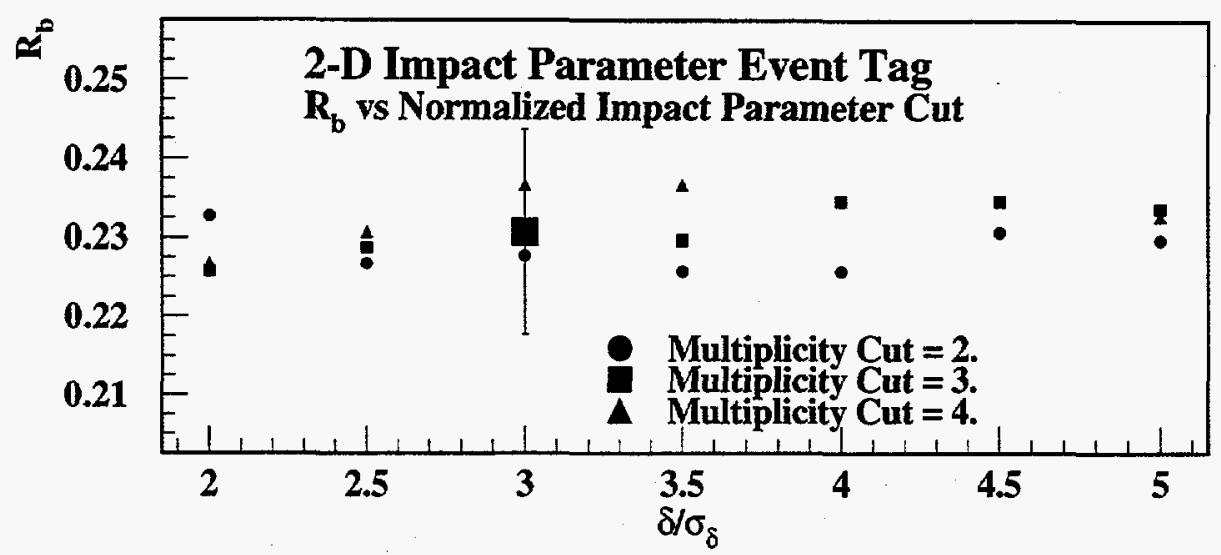

Figure 8.6: Variation in $R_{b}$ result with changes in tagging cuts.

\begin{tabular}{|l|l|}
\hline 1992 Result & $R_{b}=0.230 \pm 0.005_{\text {statistical }}$ \\
1993 Result & $R_{b}=0.230 \pm 0.009_{\text {statistical }}$ \\
\hline
\end{tabular}

Table 8.1: Event tag $R_{b}$ measurements.

\subsubsection{Detector Systematics}

\section{- Tracking Efficiency Corrections}

Tracking efficiency systematics result from the uncertainty in the corrections applied to the Monte Carlo to obtain the correct yield of quality tracks. To estimate the systematic error associated with the $p, \phi$ and $\cos \theta$ dependence of the track efficiency corrections, the change in $R_{b}$ when it is remeasured with these dependencies averaged out relative to the nominal $R_{b}$ result is used. The

\begin{tabular}{|l|c|c|}
\hline Parameter & 1992 Value & 1993 Value \\
\hline$\epsilon_{b}$ & $(61.7 \pm 0.8) \%$ & $(62.7 \pm 0.4) \%$ \\
purity of b tag & $(89 \pm 0.6) \%$ & $(89 \pm 0.3) \%$ \\
$\epsilon_{c}$ & $(9.1 \pm 0.5) \%$ & $(8.9 \pm 0.3) \%$ \\
$\epsilon_{u d s}$ & $(0.20 \pm 0.04) \%$ & $(0.3 \pm 0.02) \%$ \\
\hline
\end{tabular}

Table 8.2: Performance of the 2-D Impact Parameter Event Tag with a cut of 3 tracks with significance $>3.0$. The uncertainties are statistical only. 
observed change is $\sim 1 \%$ and is taken as the systematic contribution. In addition, a variation of 0.3 tracks per event between two halves of the 1993 data is unexplained by the known deficiencies in the Monte Carlo simulation. Changes in $R_{b}$ due to a \pm 0.3 quality track event multiplicity variation in the Monte Carlo is included as a systematic error.

\section{- Tracking Resolution}

The tracking resolution systematic comes from the various corrections to the impact parameters in the Monte Carlo. These include degradation of the $r z$ resolution and the $\phi$ dependent shift corrections, as explained in section 7.4. These predominantly affect only the core of the impact parameter distribution. The uncertainty is taken as the change in $R_{b}$ that results from not applying these corrections in turn then added in quadrature.

\section{- Primary Vertex Position}

The uncertainty coming from the non-Gaussian tails of the residual primary vertex position is taken from the change in $R_{b}$ resulting from adding these tails as described in Sect. 4.

\section{- Event Selection Bias}

While no flavor bias due to the event selection was observed, the uncertainty on the measurement of the bias is taken as a systematic.

The combined detector and primary vertex simulation uncertainty is shown in Table 8.3 .

\subsubsection{Physics Systematics}

Systematics due to the uncertainty on physics simulation parameters are estimated using an event weighting scheme. The weights are assigned the ratio of the distribution obtained for a variable(e.g. $B$-hadron lifetime) corresponding to the physics parameter(e.g. the average $B$-hadron lifetime) when it has been changed to the limit of its uncertainty and the distribution obtained at the nominal value of the 


\begin{tabular}{|l|c|}
\hline $\begin{array}{l}\text { Detector } \\
\text { Modelling }\end{array}$ & Uncertainty (\%) \\
\hline Tracking/Linking Efficiency & 2.9 \\
Tracking Resolution & 0.5 \\
Beam Position Tails & 0.5 \\
Trigger/Event Selection & 0.5 \\
\hline \multicolumn{1}{|c|}{ Total } & 3.1 \\
\hline
\end{tabular}

Table 8.3: Summary of detector systematics for the 2-D Impact Parameter Event Tag with a cut of 3 tracks with significance $>3.0$.

parameter. If $\langle\xi\rangle$ is the parameter, $\xi$ the variable, and $f_{\langle\xi\rangle}^{\text {nominal }}$ and $f_{\langle\xi\rangle}^{\text {varied }}$ are the nominal and varied distributions, then the weight $(W)$ for an event characterized by $\xi$ is $W(\xi)=\frac{1}{A} \times \frac{f_{(\xi)}^{\text {varied }}(\xi)}{f_{\langle\xi\rangle}^{\text {nominal }}(\xi)}$. The normalization constant $A=\frac{\sum_{i}^{\text {all }} \text { events }}{N_{i}(\xi)}$ insures that the sum of the weights equals the number of events being weighed. All normalization factors are within $1 \%$ of 1.0. The systematic is taken as half the difference in $R_{b}$ obtained by weighing the Monte Carlo with the parameter $1 \sigma$ high and $1 \sigma$ low. Using the event weighting scheme the following physics systematics are studied.

\section{- B-Lifetime}

The average $B$-hadron lifetime is varied by $\pm 0.10 \mathrm{ps}$ for $B$ mesons and $\pm 0.30 \mathrm{ps}$ for $B$-baryons. These variations are based on the current exclusive $B$-lifetime measurement uncertainties.[14]. This uncertainty will be one of the dominant contributions to the $R_{b}$ systematic at tight cuts.

\section{- $B$ and $C$ fragmentation}

The uncertainty from the simulation of heavy flavor fragmentation is from varying the mean energy of the Peterson distribution and from the difference in the nominal result and that obtained using the Bowler fragmentation model with the same mean energy. The $\epsilon$ parameter of Peterson fragmentation function to correspond to $\delta\left\langle x_{E}\right\rangle= \pm 0.025$ and \pm 0.021 for $c$ and $b$ quarks respectively. to account for the complexities and inability to directly determine the fragmentation process a generous variation of $2 \sigma$ is used[46]. The change in the result 


\begin{tabular}{|l|c|}
\hline$B$ Physics Simulation Systematic & $R_{b}$ Uncertainty (\%) \\
\hline $\begin{array}{l}\text { B-lifetimes } \\
\left(\tau_{\text {Bmeson }}=1.55 \pm 0.10 \mathrm{ps}, \tau_{\text {Bbaryon }}=1.10 \pm 0.30 \mathrm{ps}\right)\end{array}$ & 3.1 \\
$b$-fragmentation & \\
$\begin{array}{l}\left.\text { (Peterson }\left\langle x_{e}\right\rangle=0.695 \pm 0.021\right) \\
b \text {-fragmentation } \\
\left(\text { Bowler vs. Peterson w } /\left\langle x_{e}\right\rangle=0.695\right)\end{array}$ & 2.2 \\
$b$-baryon production & 0.2 \\
$\begin{array}{l}(8.9 \pm 3.0 \%) \\
\text { B-decay to } D^{+}\end{array}$ & 0.6 \\
$\begin{array}{l}\text { B-decay multiplicity } \\
( \pm 0.25 \text { tracks per } B \text { decay) } \\
\text { B-model } \\
(\text { exclusive phase space vs. tuned LUND } 6.3)\end{array}$ & 0.3 \\
\hline \multicolumn{1}{|c|}{ Total } & 2.6 \\
\hline
\end{tabular}

Table 8.4: Summary of $b$-physics systematics for the 2-D Impact Parameter Event Tag with a cut of 3 tracks with significance $>3.0$.

obtained from modified Bowler fragmentation function[52] for $c$ and $b$ quarks to that obtained results obtained using the Peterson function for the same $\left\langle x_{E}\right\rangle$. The significant difference in the fragmentation function shape between the two gives assurance that any model dependence has been fully account for.

\section{- $B$-decay multiplicity}

The mean charged multiplicity per $B$-hadron decay is varied by \pm 0.25 tracks. This corresponds to a $\sim 2 \sigma$ error on $B$ meson decay average charged multiplicity measured at the $\Upsilon(4 s)$ [53][54]. The lesser known $B_{s}$ and $B$-baryon decay multiplicities are covered by this variation.

\section{- Charm hadron production in $b \bar{b}$ events}

The contamination of the tag from charm decays results from the long lifetimes of the charm hadrons. Among the charm hadrons the $D^{+}$has the longest lifetime (see Table 6.3) and contributes the most to the impurity. The $B$ decay $D^{+}$ 


\begin{tabular}{|l|c|}
\hline Charm Hadron Species & Production Uncertainty \\
\hline$D^{0}$ & $\pm 5.3 \%$ \\
$D^{+}$ & $\pm 3.7 \%$ \\
$D_{s}$ & $\pm 7.0 \%$ \\
$\lambda_{c}$ & $\pm 0.5 \%$ \\
\hline
\end{tabular}

Table 8.5: Variations applied to charm hadron production.

production ratio $\frac{\Gamma\left(B \rightarrow D^{+} X\right)}{\Gamma(B \rightarrow a l l)}$ is varied by $6 \%$ absolute to account for production of all species of charmed hadrons. The current experimental uncertainty for $D^{+}$ production is $B$ decays is $\pm 4 \%$ [14].

\section{- B-baryon production}

The $B$-baryon has a significantly shorter average lifetime(1.1 ps) compared to the $B$-mesons $(1.55 \mathrm{ps})$. The $B$-baryon production rate in $b \bar{b}$ events is varied by $3 \%$ to study the effect that these differing lifetimes have on the tag.

\section{- $B$-Decay simulation}

An alternate model which is also tuned to the various measurements at $\Upsilon(4 s)$ but with decay product momenta for hadronic $B$ decay modes distributed by pure phase space was used to test the effects of differing kinematic distributions.

\section{- Charm hadron production in $c \bar{c}$ events}

The fractions of $D^{0}, D^{+}, D_{s}$ and $\lambda_{c}$ in charm events are varied according to the recommendations of reference [39]. The variations are shown in Table 8.5.

\section{- Charm decay multiplicities}

The average charged track multiplicities of the various species of charm hadrons are varied. The variations of the $D^{0}$ and $D_{s}$ decay charged multiplicities are according to the MARK-III measurement [40]. For $D^{+}$, the discrepancy between the Monte Carlo and MARK-III measurement is used. The discrepancy is due a deficit of 1-prong $D^{+}$decay modes in the Monte Carlo. The variations, which are shown in Table 8.6, are applied to $b \bar{b}$ as well as $c \bar{c}$ events in the determination of $R_{b}$ variations. 


\begin{tabular}{|l|c|}
\hline Charm Hadron Species & Decay Multiplicity Uncertainty \\
\hline$D^{0}$ & $\pm 0.06 \%$ \\
$D^{+}$ & $\pm 0.10 \%$ \\
$D_{s}$ & $\pm 0.31 \%$ \\
$\lambda_{c}$ & $\pm 0.40 \%$ \\
\hline
\end{tabular}

Table 8.6: Variations applied to charm hadron decay multiplicities.

\section{- Strange Particle Production}

The production of long lived strange particles in the fragmentation process can be a significant cause of tagging light flavor events. This effect is studied from the event fragmentation $K^{0}$ and $\Lambda$ production multiplicity by varying the Monte Carlo fragmentation $s \bar{s}$ popping suppression factor relative to $u \bar{u}$ and $d \bar{d}$ popping in the range $0.30 \pm 0.03$.

- $u d s$ Charged Particle Multiplicity The influence due to the uncertainty associated with the fragmentation of $u d s$ events is checked further by a variation of the average event charged multiplicity by \pm 0.3 tracks for $u d s$ events corresponding to the typical measurement error of the event mean charged multiplicity for all $Z^{0} \rightarrow$ hadrons events [47].

\section{- Gluon splitting in light quark events}

A crude estimate is made for the effect of heavy quark pair production due to gluon splitting. This is done by simply varying the $g \rightarrow b \bar{b}$ and $g \rightarrow c \bar{c}$ rates by $50 \%$ from that predicted by JETSET.

- Charm branching fraction $\left(R_{c}\right)$

The effect of the present experimental uncertainty in the $Z^{0} \rightarrow c \bar{c}$ branching fraction of $\delta R_{c}= \pm 0.017$ is also included as a systematic error.

\section{- Jet Axis Finding}

The uncertainty introduced by Monte Carlo jet axis modelling is determined by varying the JADE jet finding algorithm parameter $y_{\text {cut }}$ from 0.01 to 0.10 . 


\begin{tabular}{|c|c|}
\hline Physics Systematic & $R_{b}$ Uncertainty (\%) \\
\hline$c$-fragmentation & \\
\hline$\left(\right.$ Peterson $\left\langle x_{e}\right\rangle$ for $\left.D^{*}=0.501 \pm 0.025\right)$ & 0.4 \\
\hline$c$-fragmentation & \\
\hline (Bowler vs. Peterson $\mathrm{w} /\left\langle x_{e}\right\rangle=0.501$ ) & 0.1 \\
\hline$c$-decay to $D^{+}$ & \\
\hline ( $\pm 5 \%$ absolute $)$ & $<0.1$ \\
\hline $\begin{array}{l}c \text { decay multiplicity } \\
s \text { production }\end{array}$ & 0.5 \\
\hline$(s \bar{s}$ popping varied by $10 \%)$ & 0.3 \\
\hline$u d s$-decay multiplicity $( \pm 0.3$ tracks $)$ & 0.1 \\
\hline$g \rightarrow b \bar{b}$ splitting $( \pm 50 \%)$ & 0.5 \\
\hline$g \rightarrow c \bar{c}$ splitting $( \pm 50 \%)$ & 0.3 \\
\hline$\Gamma\left(Z^{0} \rightarrow c \bar{c}\right)$ & \\
\hline$\left(R_{c}=0.171 \pm 0.017\right)$ & 1.0 \\
\hline $\begin{array}{l}\text { Jet axis modelling } \\
\left(\mathrm{JADE} y_{\text {cut }} \text { varied from } 0.01 \text { to } 0.10\right)\end{array}$ & 0.8 \\
\hline Total & 1.7 \\
\hline
\end{tabular}

Table 8.7: Summary of physics systematics, other than those directly resulting from the $b$-physics simulation, for the 2-D Impact Parameter Event Tag with a cut of 3 tracks with significance $>3.0$.

The systematic contribution to $R_{b}$ caused by each of these variations is given in Tables 8.4 and 8.7. The fragmentation systematics represent two independent means in which the functional form of the fraction of the quark energy carried by the hadron may vary. It is possible to error in the shape of the function while still having the correct average energy fraction. The variation of the average energy for the Bowler function was not performed. It is expected to yield a similar variation to that obtained from the Peterson function. The two systematics are thus combined in quadrature. The gluon splitting systematics are independent of the fragmentation. The radiation of a gluon alters the energy of the quark but not the fraction of the quark energy carried by the hadron. The two gluon splitting systematics are correlated through choice of simulation model, and QCD scale. Thus, since the effect of the $c$ and $b$ gluon splitting on the value of $R_{b}$ are cumulative the sum of the splitting systematics 
is used and then combined in quadrature with the other systematics. There is also no direct correlation between the track multiplicity of the $B$ and $D$ hadron decays and the specific $B$ or $D$ hadron type. These are then each taken as independent uncertainties and combined in quadrature with the other systematics. The remaining systematics are clearly independent.

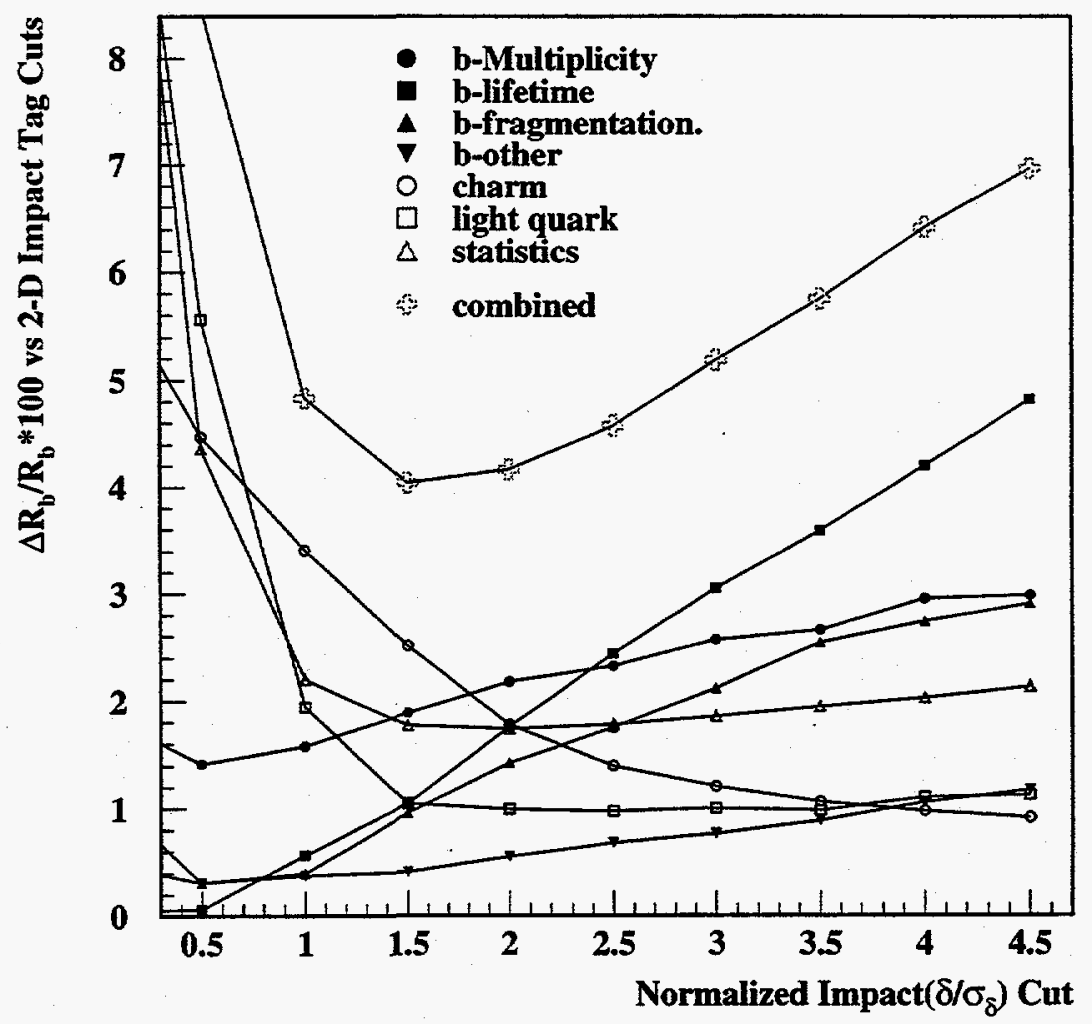

Figure 8.7: Plot of the contributions to the uncertainty in $R_{b}$ versus the significance cut at a multiplicity cut of 3 .

\subsection{Verifications}

Improper detector or physics simulation would be reflected in a dependence of the $R_{b}$ result on the $b$-tag cuts. At cuts of low $S$ and low $N_{S}$ the result becomes sensitive to simulation of the detector resolution and efficiencies. On the other end of the 


\begin{tabular}{|ccccc|c|}
\hline \multicolumn{3}{|c|}{ SLD $\phi$} & Quadrant & $R_{b}$ \\
\hline$\frac{-\pi}{4}$ & $<$ & $\phi$ & $<$ & $\frac{\pi}{4}$ & $0.234 \pm 0.009$ \\
$\frac{\pi}{4}$ & $<$ & $\phi$ & $<$ & $\frac{3 \pi}{4}$ & $0.231 \pm 0.009$ \\
$\frac{3 \pi}{4}$ & $<$ & $\phi$ & $<$ & $\frac{5 \pi}{4}$ & $0.230 \pm 0.009$ \\
$\frac{5 \pi}{4}$ & $<$ & $\phi$ & $<$ & $\frac{-\pi}{4}$ & $0.225 \pm 0.009$ \\
\hline
\end{tabular}

Table 8.8: Measurements of $R_{b}$ from the 2-D Impact Parameter Event Tag versus orientation of the thrust axis. The errors are statistical only.

spectrum, high $S$ and high $N_{S}$ the result becomes sensitive to the physics simulation. All of the variations are contained within the uncertainty of the value at the chosen cut.

\subsubsection{Event Orientation}

It is possible that a poorly functioning section of the detector could cause unsimulated high impact parameters for tracks passing through that region or unsimulated efficiency problems. To check for this the $R_{b}$ result versus orientation of the events is studied by measuring $R_{b}$ for events with thrust axis direction in different $\phi$ regions separately. The ambiguity in the thrust direction is removed by taking the thrust axis to always point along the positron beam direction. The result in each $\phi$ region is given in Table 8.8 and the results in different $\phi$ regions are statistically consistent with each other.

\subsection{Final Result}

A summary of the systematics is shown in Fig. 8.7 for a multiplicity cut of 3 . The minimum total uncertainty occurs near $S=2.0$. This is mainly being driven by the $\tau_{\mathcal{B}}$ uncertainty. To reduce the influence of any unsimulated resolution effects, which would be reflected in the core of the distribution, a cut of $S=3.0$ with $N_{S}=3$ is selected.

Combining the 1992 and 1993 results from Table 8.1 and including the physics and detector systematics the following result is obtained:

$$
R_{b}=0.230 \pm 0.004_{\text {statistical }} \pm 0.013_{\text {systematic }}
$$




\section{Chapter 9}

\section{The Lifetime Probability Tag}

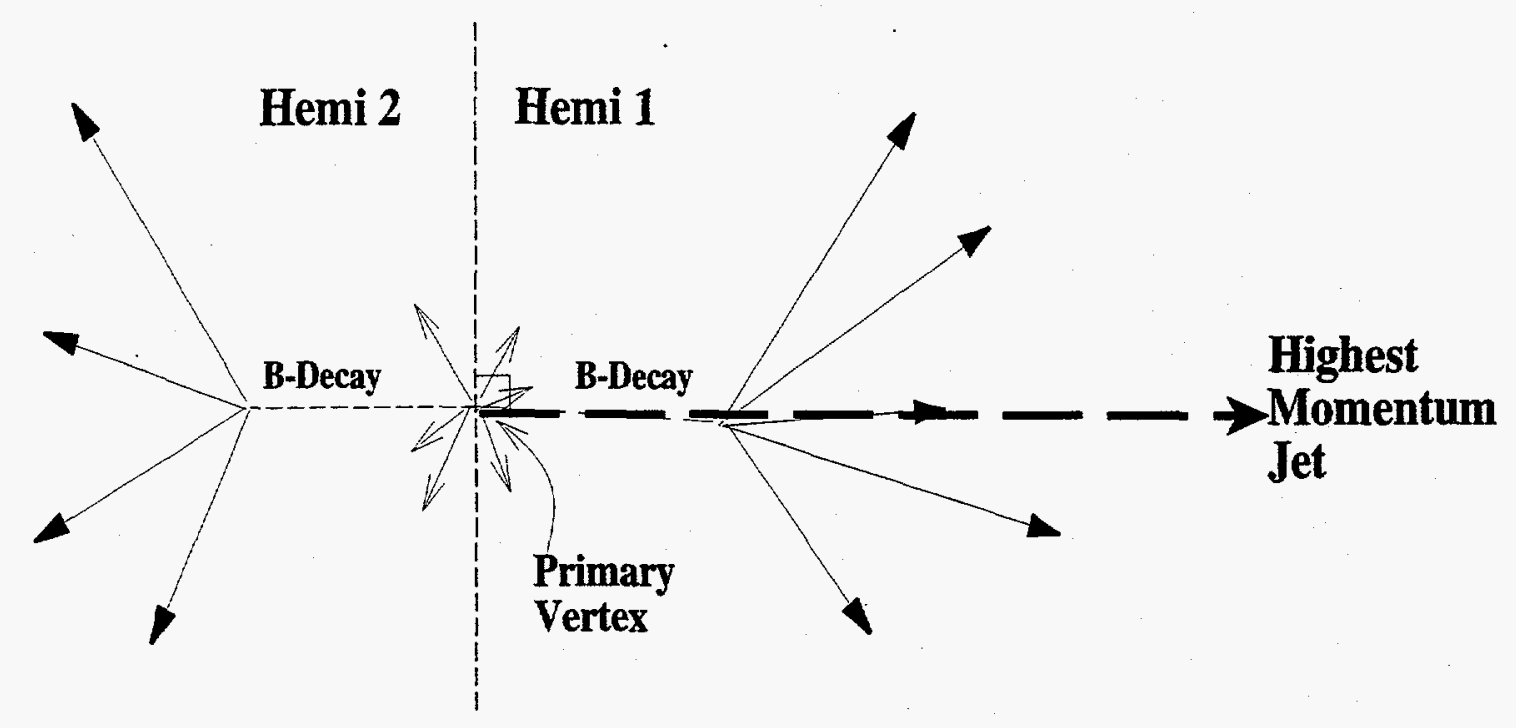

\subsection{Introduction}

In the previous chapter, the event tag method was presented. As demonstrated, it is already systematics limited due to uncertainties on $b$-lifetime, $b$-fragmentation, and the $b$-decay multiplicity. The measurements that contribute to these errors are not expected to significantly improve in the near future and a method less sensitive to the $b$-decay simulation must be used to make further progress. Such a method has been developed by ALEPH[8]. In this section, the lifetime probability tag method, a 
variation of the ALEPH technique, is described. This method compares the rate at which an event hemisphere is $b$-tagged to the rate at which both hemispheres of an event are $b$-tagged, to measure $\epsilon_{b}$ simultaneously with $R_{b}$. Ideally, this would entirely remove any dependence on the $B$-decay simulation. Contributions from physics and detector effects, that correlate one hemisphere with the other are determined from the $B$-model and introduce a residual systematic that is small compared to that coming from the tagging efficiency for $c$ and $u d s$ decays. The result from this measurement is competitive with many of those from LEP.

For this method, the tag variable $\chi$ (see Sect. 5.2) is used to employ the full 3-D information provided by the tracking systems. The addition of the $r z$ information is needed to maintain a high $b$-tagging purity while avoiding large statistical uncertainties. The result of adding the $r z$ impact information for tagging an event is an improvement of approximately $7 \%$ over the 2-D impact parameter tagging efficiency while achieving the same tagging purity. The distribution of $\chi$ for $b, c, u d s$ and all Monte Carlo and data is shown in Fig. 9.1.

The results will be shown to complement the event tag results because the sources of systematics are considerably different. It can ultimately be the best measurement with the data from future SLD runs, using the upgraded vertex detector, VXD3.

\subsubsection{Outline of the Probability Tagging Procedure}

Hemispheres containing a $b$-decay will be identified by a cut on the probability that the collection of tracks in a hemisphere are consistent with coming from the measured primary vertex position; hence the term "probability tag". The hemispheres in the event are defined by the highest momentum jet in the event. First, each track is assigned a probability for being consistent with the measured primary vertex position. Then these are combined to form the hemisphere probability. A hemisphere is $b$-tagged by requiring that the hemisphere probability be small, that is, below a selected cut-off. Finally, $R_{b}$ is measured from the rate of events in which one and both hemispheres are tagged. 

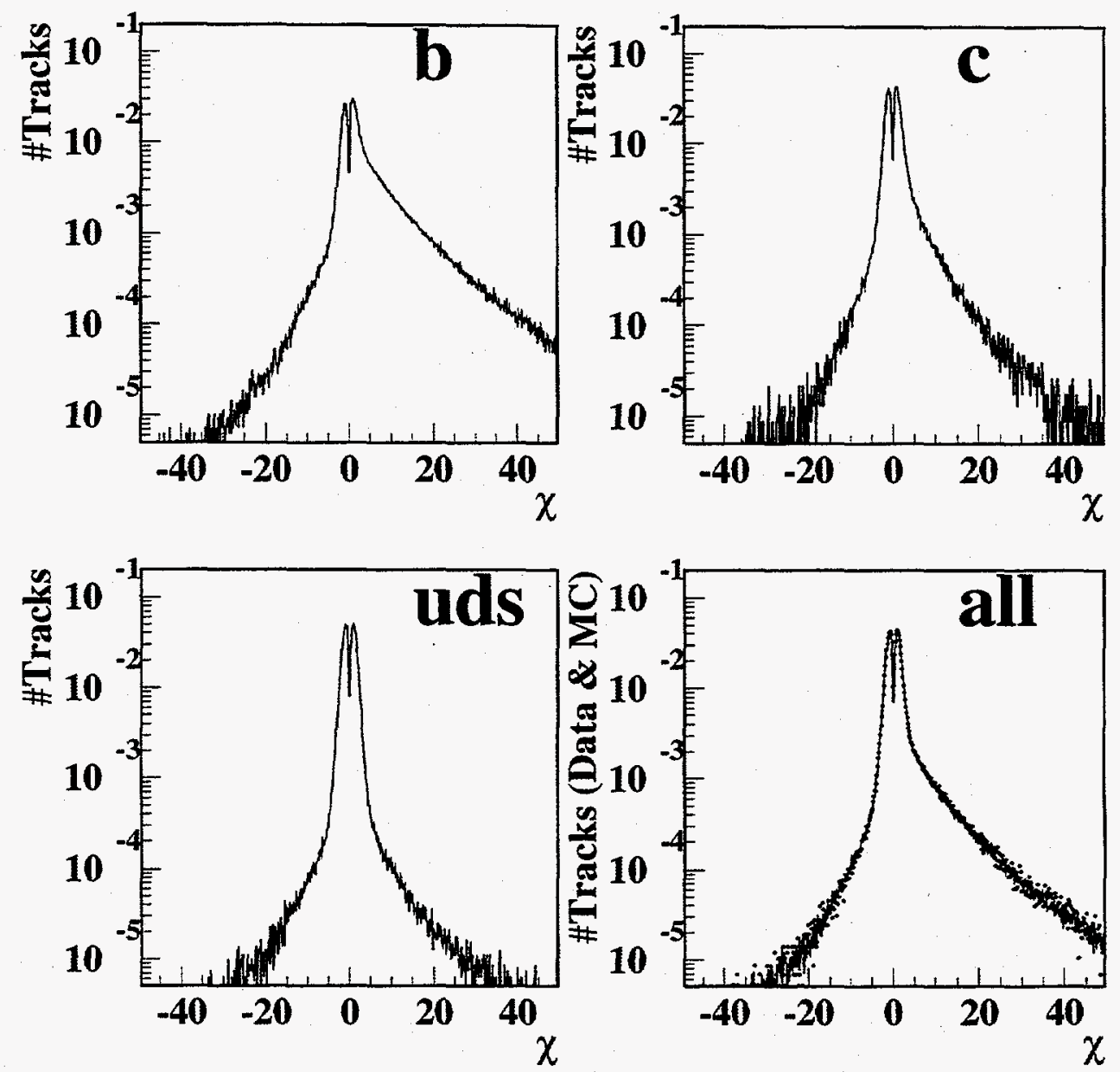

Figure 9.1: Distributions of the 3-D significance variable $\chi$ for $\mathrm{b}, c$ and $u d s$ flavored events.

First, we start by determining the probability that a track is consistent with coming from the primary vertex. This is derived from the distribution of $\chi$ for light quark events. The reason for using the light quark events is that one needs a representation of the background excluding the lifetime information of $b$ and $c$ hadrons. Next, the probabilities for tracks within a hemisphere are combined to obtain the probability that the collection of tracks in the hemisphere are consistent with the primary vertex. 


\subsection{Event and Track Selection Modifications}

Modifications to the event and track selection cuts discussed in Chapter 7 were made to reduce hemisphere correlations and to exploit the extra information provided by the $r z$ dimension. These are described in the following sections.

\subsubsection{Event Selection and Counts}
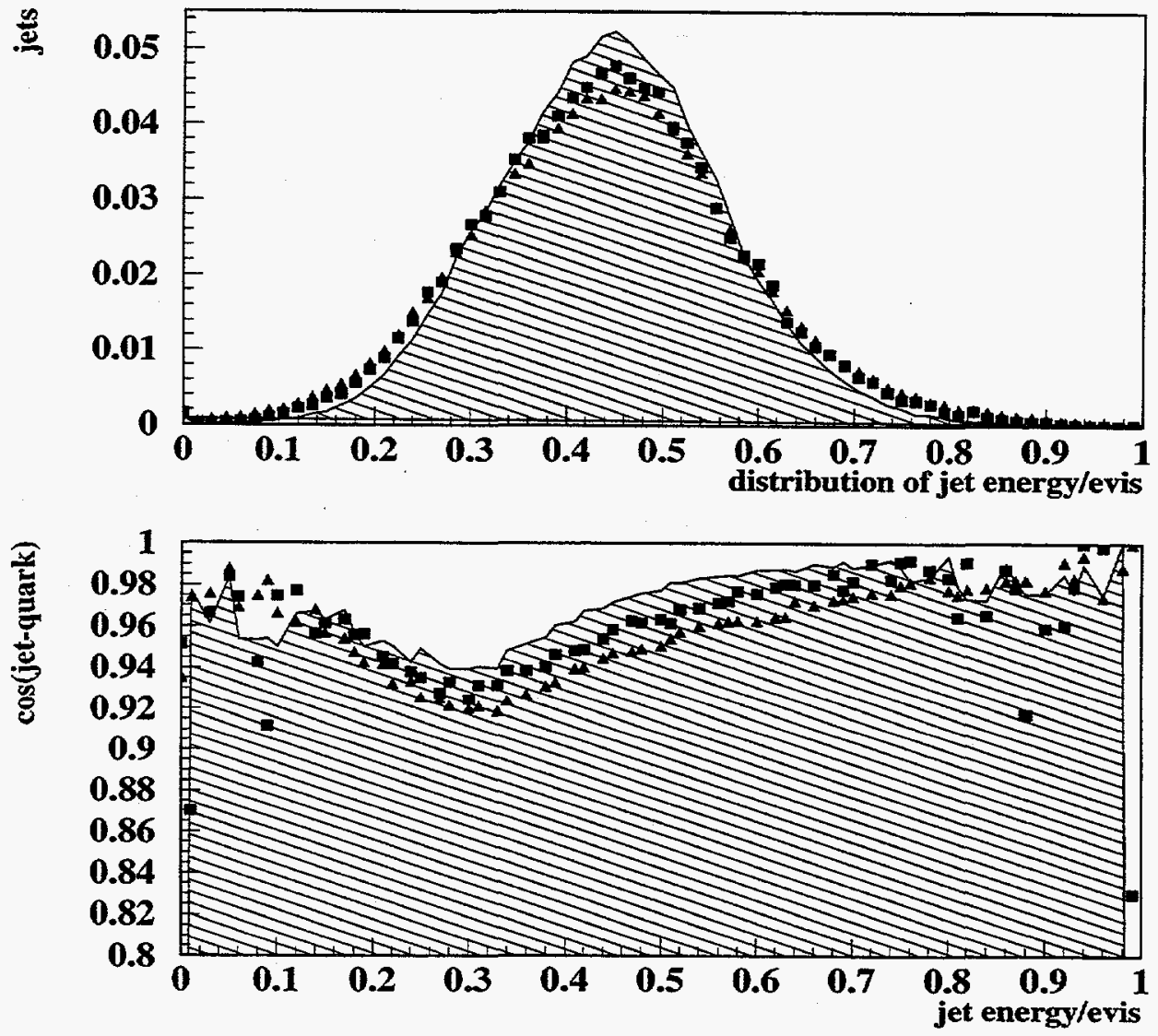

Figure 9.2: The distribution of the energy in the first two jets of the events relative to the total visible energy and the dot product of the quark and jet direction as a function of this. 
The event selection is identical to that used for the 2-D Impact Parameter Event Tag with the extra requirement that at least two jets with energy $>10 \%$ of the visible energy must exist in the event and only two and three jet events are used. This helps to eliminate jets from gluon radiation that would result in a poor estimation of the $B$-hadron flight direction. The distribution of the fraction of energy, relative to the visible energy, in the two highest momentum jets is shown in Fig. 9.2. The cosine of the angle between the jet direction and associated quark direction is also shown in Fig. 9.2. The dip near a fractional energy of 0.3 is from the presence of a hard radiated gluon.

The choice of $y_{\text {cut }}=0.02$ is based on a similar analysis to that described in Sect. 5.1.1 which found the cut that maximizes the fraction of $B$-decay tracks correctly signed. The combination of this $y_{c u t}$ and the jet count are based on retaining a high fraction of the events while also having a good estimate of the $B$-hadron direction. At a $y_{\text {cut }}=0.005$ and keeping only two jet events the average cosine of the angle between the highest momentum jet direction and nearest $B$-hadron is 0.997 . This is $\sim 0.025$ better than what is obtained using the analysis cuts. However, only $0.17 \%$ of the events are retained. The distribution of events retained for a given $y_{c u t}$ and jet count cut and the angular accuracy are shown in Fig. 9.3. If one requires that at least $80 \%$ of the events are retained then the allowed cuts are as shown in the lower figure of Fig. 9.3. With this requirement the chosen $y_{\text {cut }}$ and jet count cuts appear as the second best choice in terms of directional accuracy for the hemisphere axis. The difference between the two most accurate solutions is small as the minimum box size represents an average cosine of 0.9 .

Data from the 1993 and 1994 SLD runs and corresponding Monte Carlo are used. To reduce the systematic uncertainty coming from the statistical uncertainty on the hemisphere correlation, extra $b \bar{b}$ samples were generated. A total of $53 \mathrm{k}$ data events, and from the Monte Carlo $83 \mathrm{k} b \bar{b}, 21 \mathrm{k} c \bar{c}$, and $73 \mathrm{k} u d s$ events are used for the analysis. 


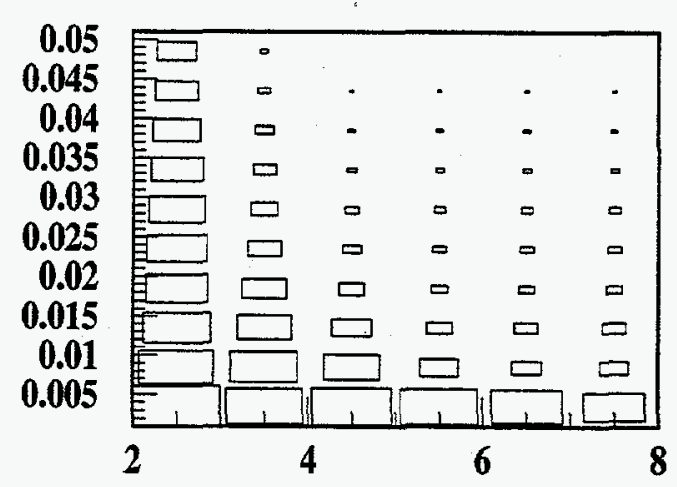

$$
\cos \theta\left(\mathbf{P}_{\text {jet }} \mathbf{P}_{\mathrm{B} \mathrm{hadron}}\right) \text { vs } \mathbf{y}_{\text {cut }} \text { and \#jets }
$$

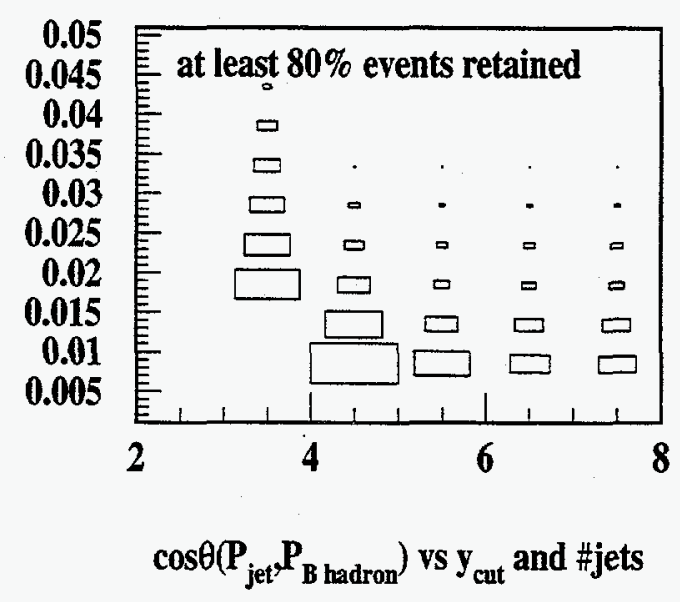

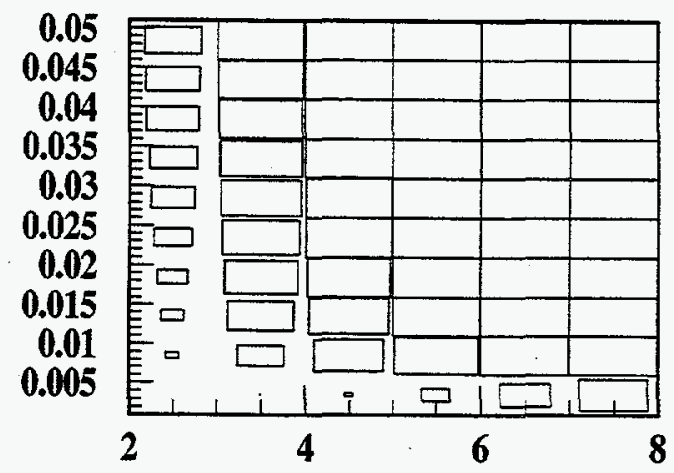

\#Events vs $\mathbf{y}_{\text {cat }}$ and \#jets

Vertical axes $=\mathbf{J A D E} \mathbf{y}_{\text {cut }}$

Horizontal axes $=$ cut on number of jets allowed

Figure 9.3: Indicators of accuracy to which the $B$-hadron direction is determined versus different jet finding and jet count cuts.

\subsubsection{Track Selection}

All of the track quality cuts for the 2-D Impact Parameter Event Tag analysis are used. Additional track cuts are applied to further help in removing contamination to the tag from very long lived particle decays. A cut on the distance of closest approach of the track to the jet axis $\left(\delta_{j}\right)$ and on the distance from the primary vertex to the point on the jet at which the track is closest $\left(P_{j}\right)$ are used. These quantities are 

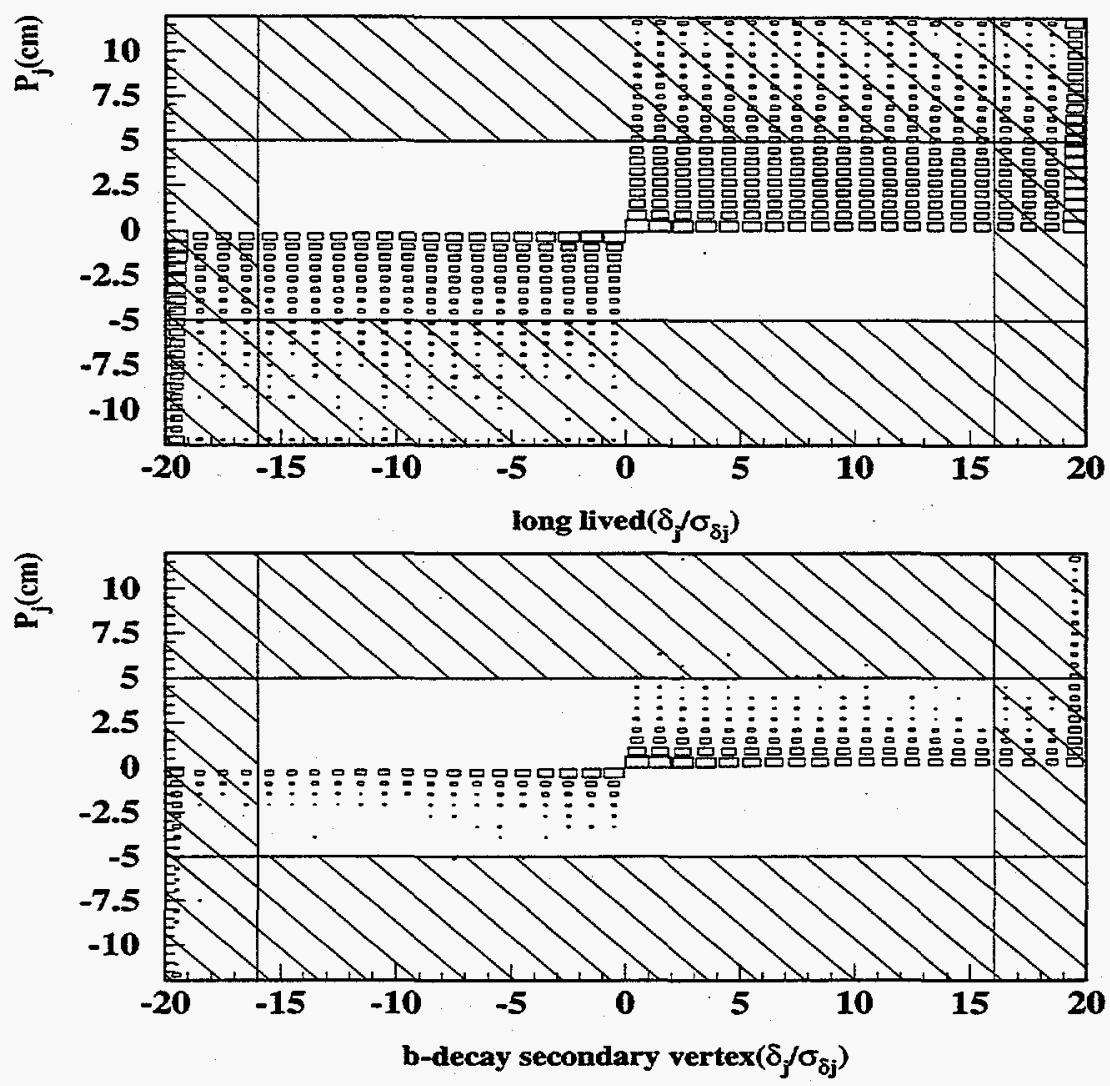

Figure 9.4: The distance along the jet axis to the point of closest approach between the track and the jet versus the distance of closest approach between the track and jet normalized by the uncertainty on the track position along the d.o.c.a. direction. This is shown for long lived particles (i.e. $K_{s}^{0}, \Lambda, \Sigma$ decay products etc...) which contaminated the signal and for tracks from the secondary $b$ vertex only which contain the desired tagging information.

illustrated in Fig. 5.7. In Fig. 9.4 one sees that tracks from very long lived particle decays which dilute the tag can be separated from the $b$-decay tracks by cutting both on $\delta_{j}$ normalized by the uncertainty on the track position $\left(\sigma_{\delta_{j}}\right)$ along the direction of $\delta_{j}$ and $P_{j}$. A cut of $16 \sigma$ on $\delta_{j e t} / \sigma\left(\delta_{j e t}\right)$ and a cut of $5 \mathrm{~cm}$ on $P_{j}$ results in $92 \%$ of the $b$ decay tracks being kept but only $45 \%$ of the tracks from long lived particle decays still remain. 


\begin{tabular}{|l|c||l|c|}
\hline Parameter & Value & Parameter & Value \\
\hline$p_{1}$ & $59.2 \times 10^{3} \pm 0.3 \times 10^{3}$ & $p_{2}$ & $0.846 \pm 0.002$ \\
$p_{3}$ & $22 \pm 11$ & $p_{4}$ & $14 \pm 3$. \\
$p_{5}$ & $810 \pm 43$ & $p_{6}$ & $3.5 \pm 0.2$ \\
$p_{7}$ & $12.1 \times 10^{3} \pm 0.3 \times 10^{3}$ & $p_{8}$ & $1.281 \pm 0.005$ \\
\hline
\end{tabular}

Table 9.1: Parameters of resolution function fit.

\subsection{Procedure}

From the $\chi$ of the track, the probability that the track is consistent with coming from background events is determined. This is accomplished by fitting the uds distribution of $\chi$ to a resolution function. The resolution function $(R(\chi))$ is formed from a factor of $\chi$ to account for phase space multiplied by Gaussians for describing the core and exponentials for describing the tails. The probability $\left(P_{\text {track }}\right)$ that one will measure the same $\chi$ or worse is given by $\int_{-\infty}^{-\chi} R / \int_{-\infty}^{0} R$. The probability is then assigned the same sign as that of the associated $\chi$. The resolution function is:

$$
R(-|\chi|)=-p_{1} \cdot \chi \cdot e^{-.5\left(\chi / p_{2}\right)^{2}}+p_{3} \cdot e^{\chi / p_{4}}+p_{5} \cdot e^{\chi / p_{6}}+p_{7} \cdot \chi \cdot e^{-.5\left(\chi / p_{8}\right)^{2}}
$$

where the $p_{i}$ variables are the parameters that are fitted. The $-\chi$ distribution for tracks in light quark events along with the fitted resolution function is shown in Fig. 9.5. The distributions of the probabilities for the tracks are shown in Fig. 9.6. Since the probabilities are derived from the $u d s$ distribution one expects the probability distribution for $u d s$ events to be flat; in sharp contrast, the tracks from $b$ events exhibit a large peak near low probability for being consistent with the primary vertex. This is due to tracks from the $b$-decay vertex that have large impacts with respect to the primary vertex. The tails are due to fragmentation tracks.

The probability that the group of tracks belonging to a hemisphere are consistent with coming from the primary vertex is formed from the product of the positive track probabilities within the hemisphere $\left(P_{\text {kemi }}=\prod_{j=0}^{N_{\text {tracks }}} P_{\text {track }}^{j}\right.$. From this the integral probability which expresses the chance that a larger $\chi^{2}$ corresponding to the set of tracks would be measured is calculated. Since one is dealing with small samples, the 


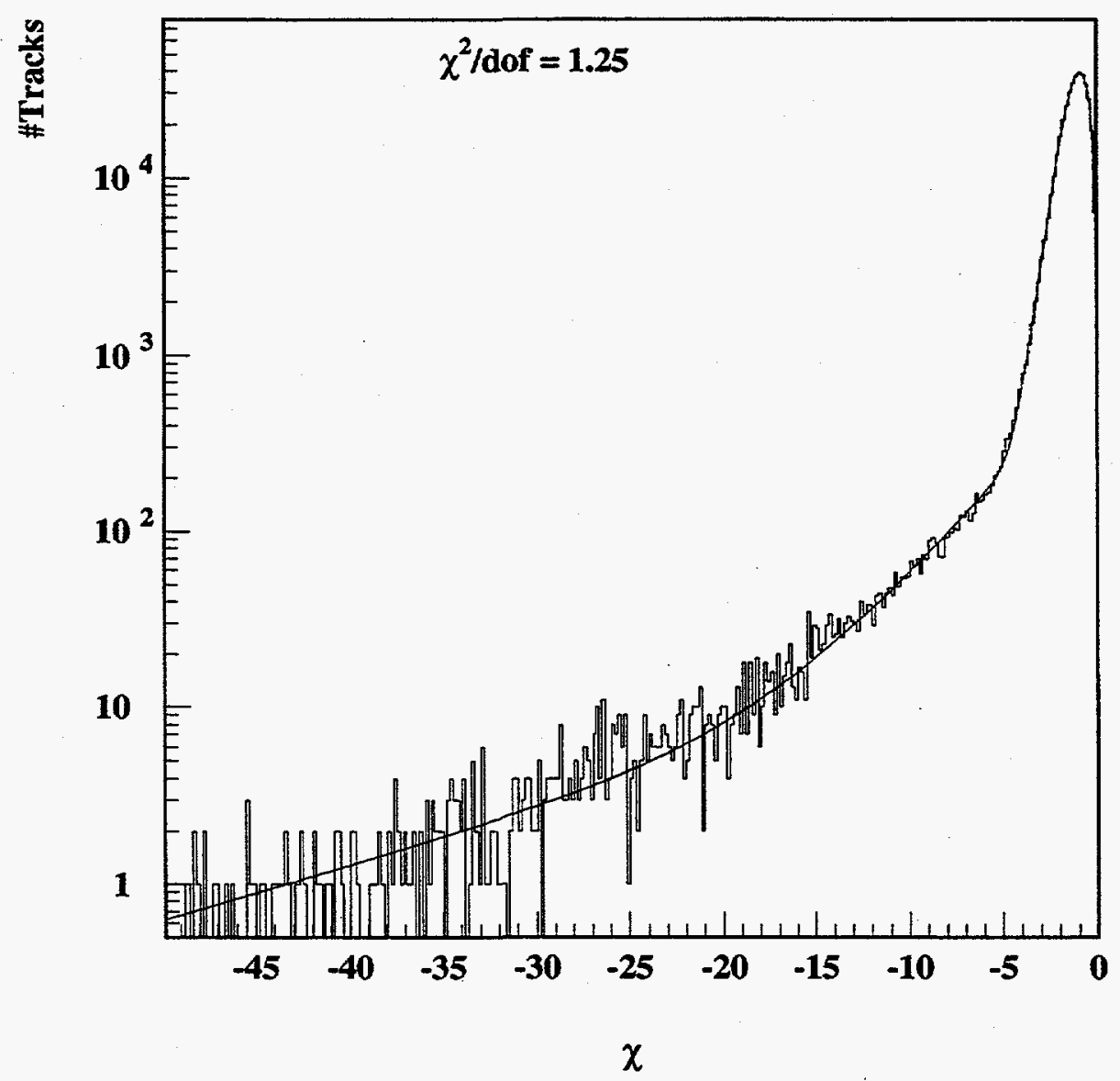

Figure 9.5: Negative $\chi$ distribution for tracks in $u d s$ events overlayed with the fit of the resolution function.

Poisson $\chi^{2}$ probability distribution[55] is used:

$$
P\left(\chi^{2} \mid \nu\right)=e^{-\frac{\chi^{2}}{2}} \sum_{j=0}^{\frac{\nu}{2}-1} \frac{{\frac{\chi^{2}}{2}}^{j}}{j !}
$$

where $\chi^{2} / 2=-\ln \left(P_{h e m i}\right)$ and $\nu$ is the number of degrees of freedom. For each track there are two degrees of freedom $\xi$ and $\eta$. Putting in the values for $\chi^{2}$ and $\nu$ one gets:

$$
\Pi_{\text {hemi }}=\left(\prod_{j=0}^{n} P_{\text {track }}^{j}\right) \sum_{k=0}^{n-1} \frac{\left(-\ln \left(\prod_{j=0}^{n} P_{\text {track }}^{j}\right)\right)^{k}}{k !}
$$



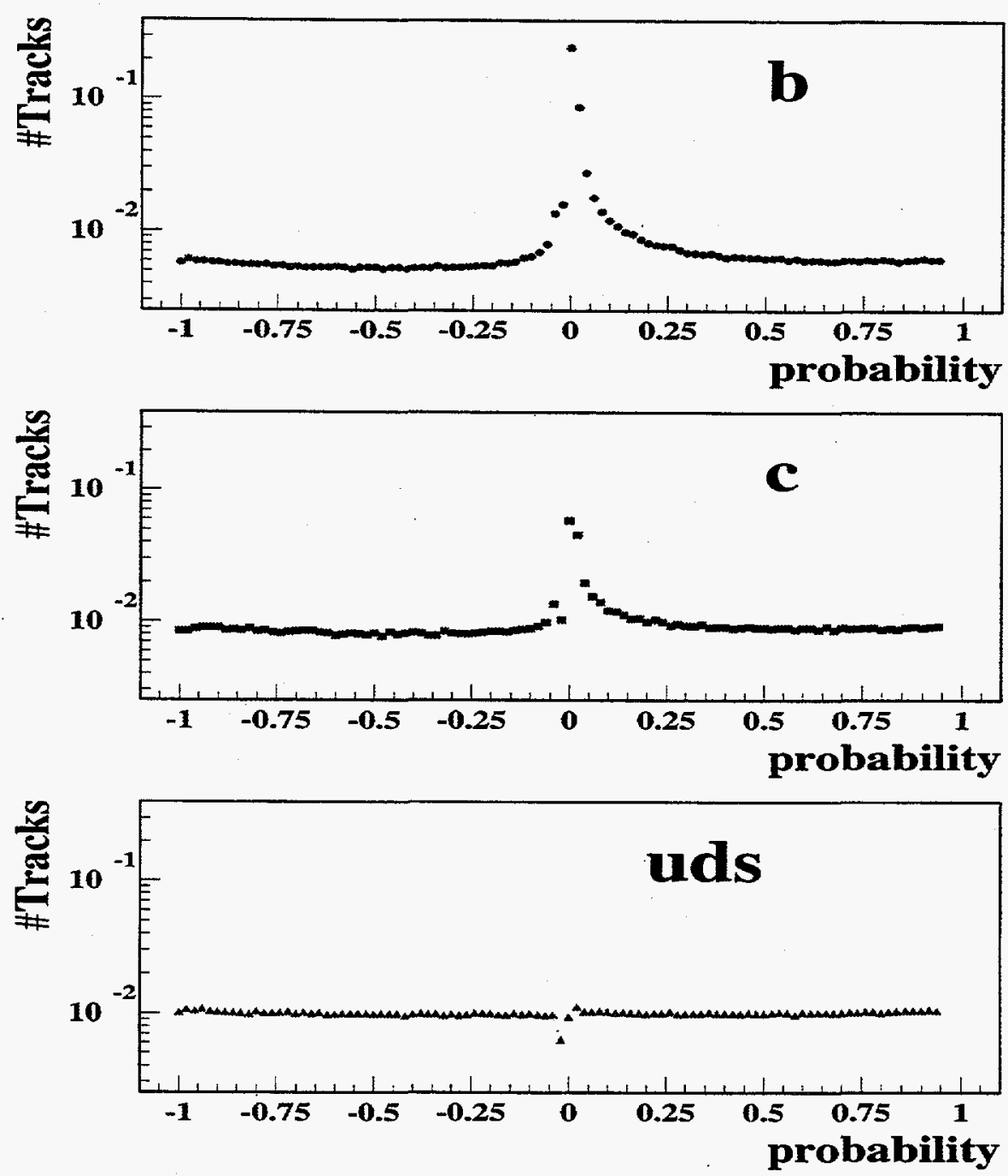

Figure 9.6: Distribution of tracks probabilities for consistency with coming from the primary vertex for $\mathrm{b}, c$ and $u d s$ events.

The distribution of these hemisphere probabilities is shown in Fig. 9.7. As lower probability cuts are required the $b$ content of the tagged sample is greatly enhanced relative to the other flavors. Note that on the scale of $-\ln \left(\Pi_{\text {hemisphere }}\right)$, as one goes to higher values (to the right) one is requiring that the tracks in the hemisphere be less likely to be consistent with the primary vertex. 


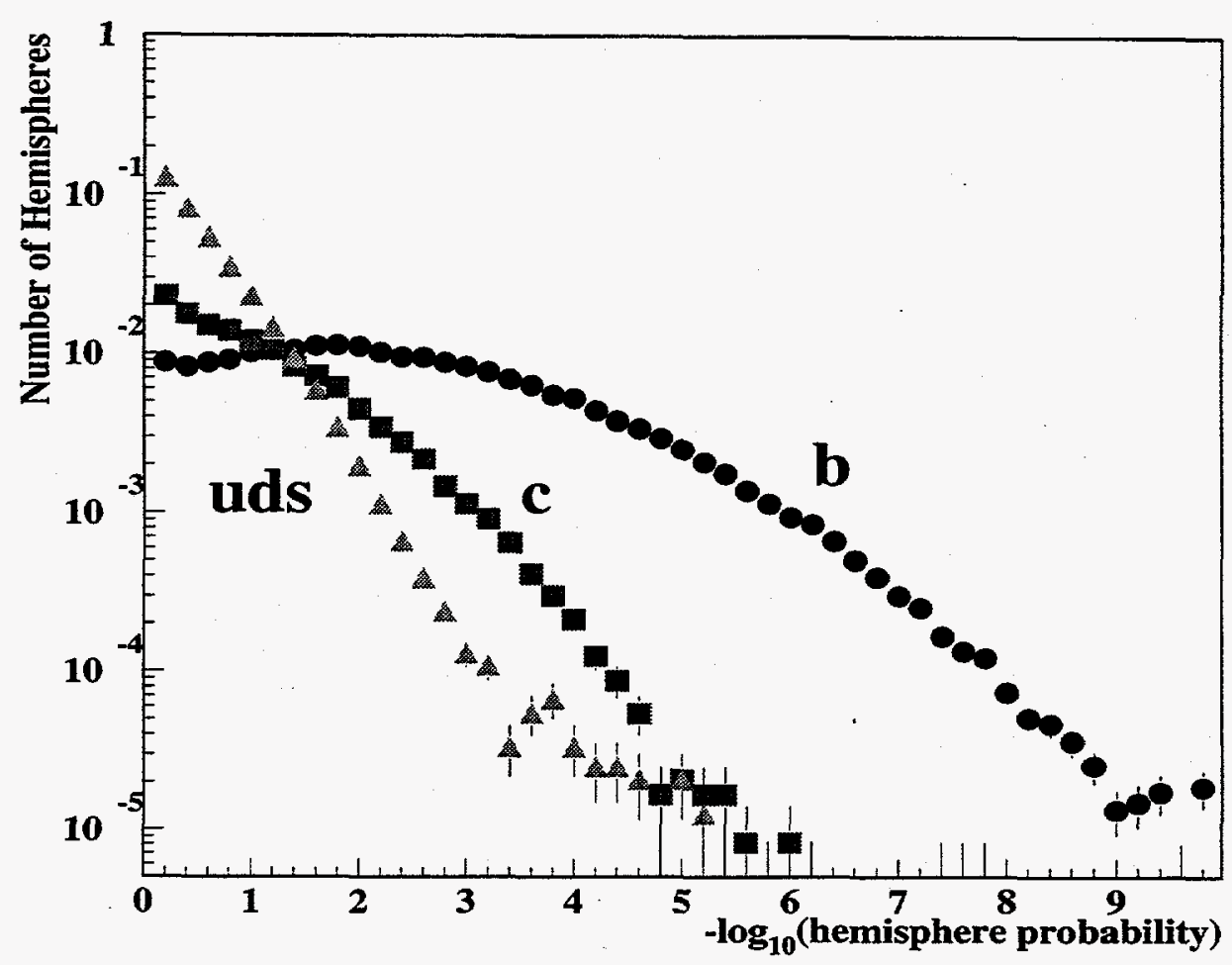

Figure 9.7: Distribution of hemisphere probabilities for $b, c$ and $u d s$ flavored events.

\subsubsection{Resolution Corrections}

The same resolution corrections, as described in Sect. 7.4, are used. After the resolution and efficiency corrections have been applied the comparison of the Monte Carlo and data for the significance variable, $\chi$, is as shown in Fig. 9.8.

\subsubsection{Determining $R_{b}$ from Tag Counts}

A hemisphere is tagged by requiring that the probability for the hemisphere $\Pi_{h e m i}<$ $P_{c u t}$. In the analysis the number of hemispheres tagged (single tags) and number of events with both hemispheres tagged (double tags) are used to simultaneously measure $\epsilon_{b}$ and $R_{b}$. As with the derivation of $R_{b}$ from the event tag one begins by expressing the net tag count in terms of the efficiencies for each of the flavor samples and the number of events in the untagged sample. The same equation that was used for the event now applies to the individual hemispheres. The number of tagged 

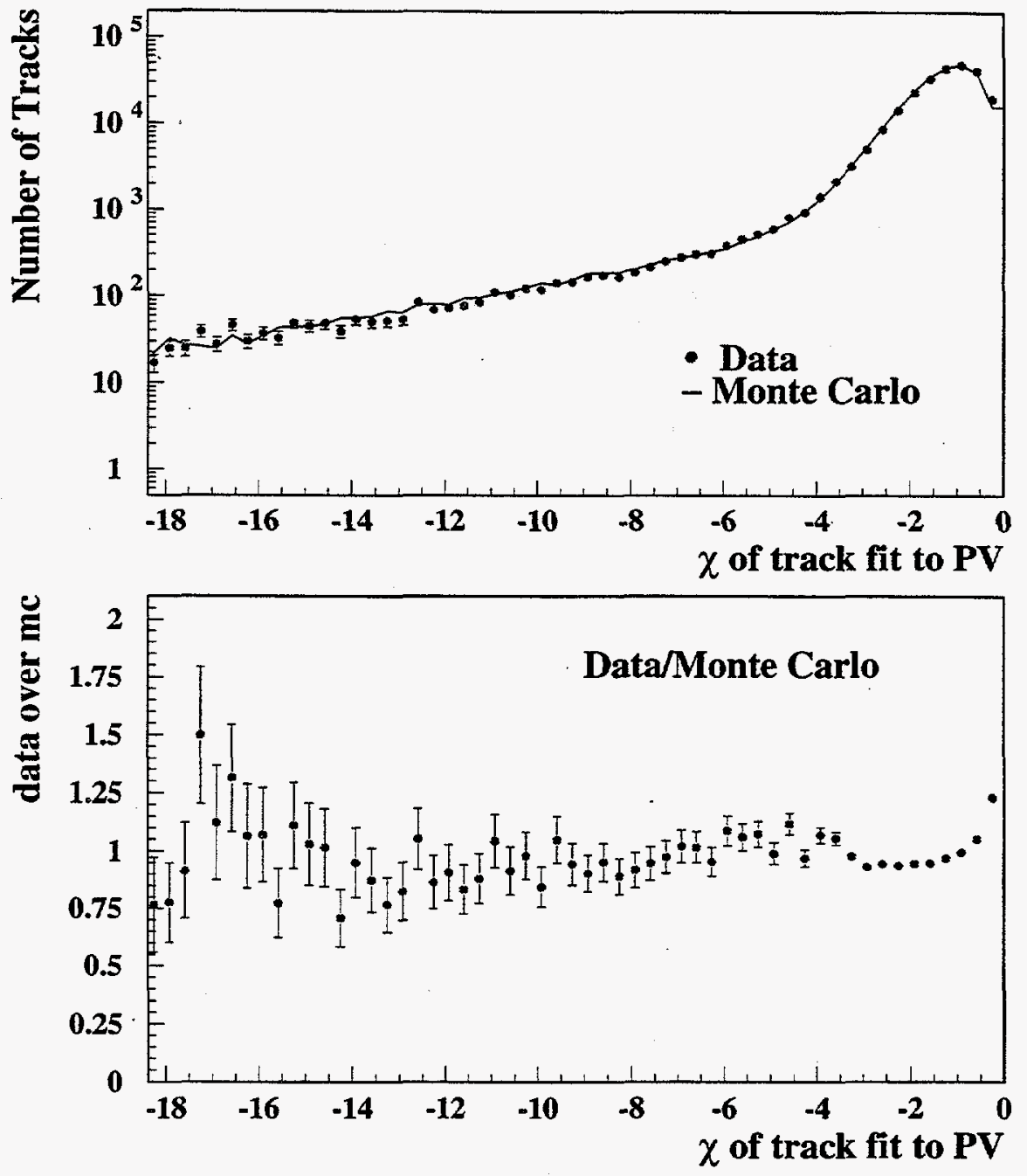

Figure 9.8: Comparison of the 3-D significance variable $\chi$ between data and Monte Carlo for tracks whose point of closest approach to the jet access is behind the primary vertex. Efficiency and minor resolution corrections have been applied.

hemispheres is given by:

$$
n_{h e m i}=\epsilon_{b} n_{b}+\epsilon_{c} n_{c}+\epsilon_{u d s} n_{u d s}
$$

However, now there is an additional equation for the tag count for the case where both hemispheres are tagged with the corresponding efficiencies for tagging both hemispheres as a function of the flavor. The number of double tagged hemisphere is 
given by:

$$
n_{\text {double }}=\epsilon_{b}^{\text {double }} n_{b}+\epsilon_{c}^{\text {double }} n_{c}+\epsilon_{u d s}^{\text {double }} n_{u d s}
$$

If no correlation existed between the hemispheres then $\epsilon_{i}^{\text {double }}=\epsilon_{i}^{2}$. The degree of correlation between the hemisphere should express the difference between these two quantities. The correlation, $\lambda$, is derived from the standard formula:

$$
\lambda=\frac{\langle x y\rangle-\langle x\rangle\langle y\rangle}{\sqrt{\left(\left\langle x^{2}>-<x>\langle x\rangle\right)\left(<y^{2}\right\rangle-<y><y>\right)}}
$$

with the variables $x$ and $y$ being $\Theta\left(P_{\text {cut }}-P\right)$ (i.e. 1 if the hemisphere passes the tag, 0 otherwise) for the first and second hemispheres respectively. The average of either variable $(x$ or $y)$ is the hemisphere tagging efficiency. The same is true for the square of the variables. The average of the product of the variables is the double hemisphere tagging efficiency. Thus, one gets:

$$
\lambda_{q}=\frac{\epsilon_{q}^{\text {double }}-\epsilon_{q}^{2}}{\epsilon_{q}-\epsilon_{q}^{2}}
$$

The terms in Eqn. 9.1 and 9.2 for the tag count can be rearranged so that everything is expressed in terms of the single hemisphere tag efficiencies and the correlation as shown below:

$$
\begin{array}{r}
n_{\text {double }}=\left(\epsilon_{b}^{2}+\frac{\epsilon_{b}^{d o u b l e}-\epsilon_{b}^{2}}{\epsilon_{b}-\epsilon_{b}^{2}}\left(\epsilon_{b}-\epsilon_{b}^{2}\right)\right) n_{b}+ \\
\left(\epsilon_{c}^{2}+\frac{\epsilon_{c}^{d o u b l e}-\epsilon_{c}^{2}}{\epsilon_{c}-\epsilon_{c}^{2}}\left(\epsilon_{c}-\epsilon_{c}^{2}\right)\right) n_{c}+ \\
\epsilon_{u d s}^{2}+\frac{\epsilon_{u d s}^{d o u b l e}-\epsilon_{u d s}^{2}}{\epsilon_{u d s}-\epsilon_{u d s}^{2}}\left(\epsilon_{u d s}-\epsilon_{u d s}^{2}\right)\left(n_{\text {hadrons }}-n_{b}-n_{c}\right) \\
n_{\text {double }}=\left(\epsilon_{b}^{2}+\lambda_{b}\left(\epsilon_{b}-\epsilon_{b}^{2}\right)\right) n_{b}+\left(\epsilon_{c}^{2}+\lambda_{c}\left(\epsilon_{c}-\epsilon_{c}^{2}\right)\right) n_{c}+ \\
\left(\epsilon_{u d s}^{2}+\lambda_{u d s}\left(\epsilon_{u d s}-\epsilon_{u d s}^{2}\right)\right)\left(n_{\text {hadrons }}-n_{b}-n_{c}\right)
\end{array}
$$


Due to the low efficiency for tagging $c$ and $u d s$ events and since the hemisphere correlations will be small, the $\lambda_{c}$ and $\lambda_{u d s}$ are second order terms that may be dropped. The resulting equation is:

$$
n_{\text {double }}=\left(\epsilon_{b}^{2}+\lambda_{b}\left(\epsilon_{b}-\epsilon_{b}^{2}\right)\right) n_{b}+\epsilon_{c}^{2} n_{c}+\epsilon_{u d s}^{2}\left(n_{\text {hadrons }}-n_{b}-n_{c}\right)
$$

$R_{b}=\frac{n_{b}}{n_{\text {hadrons }}}$ can be solved for with the dependence on $\epsilon_{b}$ removed by combining the equations for the number of hemisphere tags and double tagged events. For $R_{b}$ this yields:

$$
R_{b}=\frac{\left(F_{s}-R_{c}\left(\epsilon_{c}-\epsilon_{u d s}\right)-\epsilon_{u d s}\right)^{2}}{F_{d}-R_{c}\left(\epsilon_{c}-\epsilon_{u d s}\right)^{2}+\epsilon_{u d s}^{2}-2 F_{s} \epsilon_{u d s}-\lambda_{b} R_{b}\left(\epsilon_{b}-\epsilon_{b}^{2}\right)}
$$

where $F_{s, d}=$ fraction of single(s)/double(d) tags. For $\epsilon_{b}$ the corresponding equation is:

$$
\epsilon_{b}=\frac{F_{d}-R_{c} \epsilon_{c}\left(\epsilon_{c}-\epsilon_{u d s}\right)-F_{s} \epsilon_{u d s}-\lambda_{b} R_{b}\left(\epsilon_{b}-\epsilon_{b}^{2}\right)}{\left(F_{s}-R_{c}\left(\epsilon_{c}-\epsilon_{u d s}\right)-\epsilon_{u d s}\right.}
$$

These equations are coupled through the correlation terms and must be solved iteratively. Initially, $R_{b}$ and $\epsilon_{b}$ are set to zero and then the equations are iterated until they converge. Convergence, within the necessary precision, is typically obtained within a couple of iterations.

To determine the inputs, a 2-D histogram of the $-\log _{10} \Pi_{h e m i}$ of one hemisphere versus the other for $b, c$ and $u d s$ events and data are used to determine the single and double hemisphere tagging efficiencies and tagging fractions. The distribution for the 2-D histogram for each of the flavors is shown in Fig. 9.9. For each of the systematics studied a corresponding set of 2-D histograms is used.

\subsubsection{Bias Corrections}

The extra event selection used in this method introduces a bias towards $b \bar{b}$ events. This is mainly due to the energy cut on the jets. The correction is modeled by introducing a coefficient that modifies the various efficiencies in the equations for 

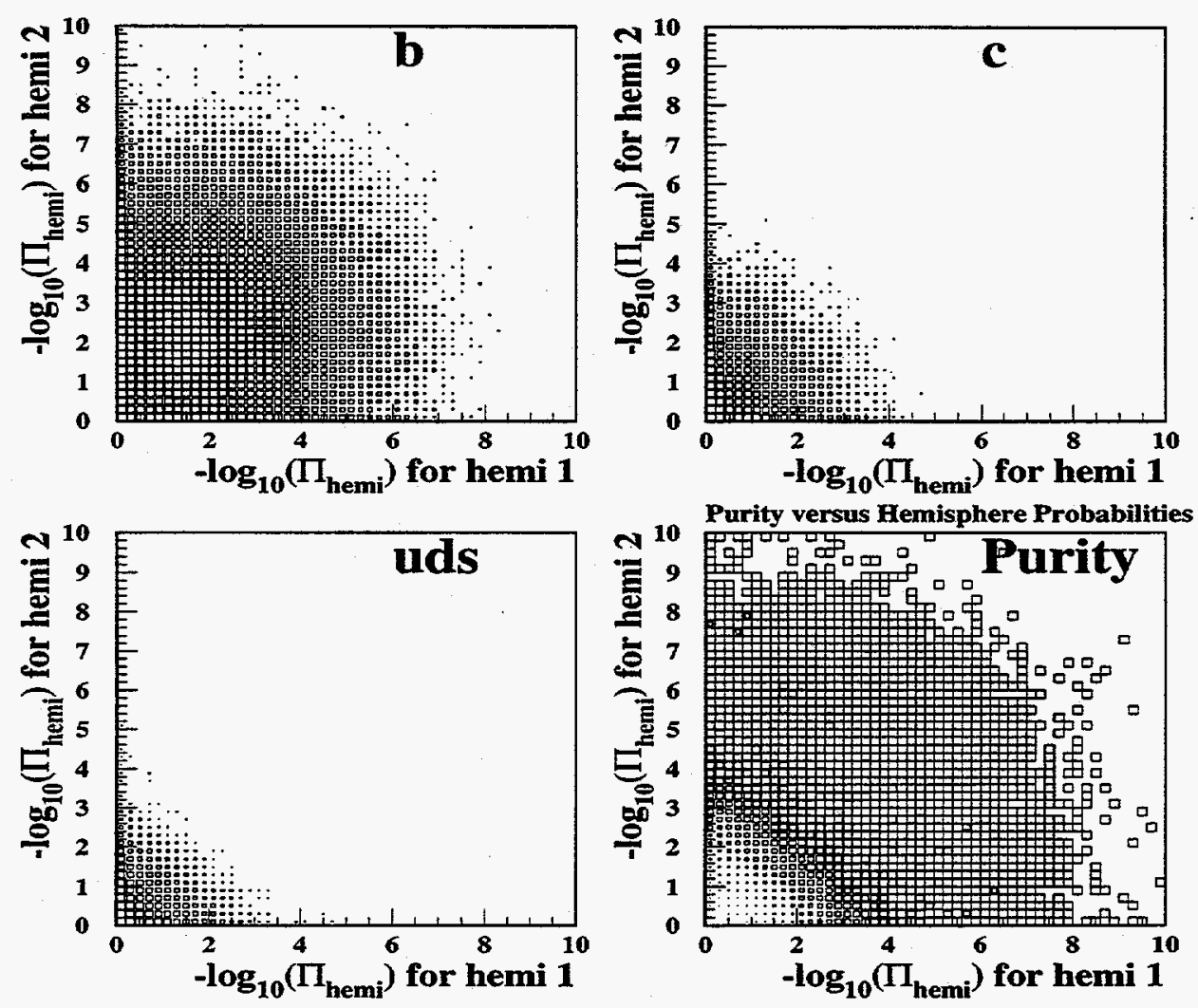

Figure 9.9: Log of number of events versus probability for each hemisphere to be consistent with the primary vertex.

the single and double tag counts from which $R_{b}$ and $\epsilon_{b}$ are rederived. The resulting equations are:

$$
\begin{gathered}
\epsilon_{b}=\frac{1}{\xi+1} \times \frac{\left(\xi R_{b}+1.0\right) F_{d}-R_{c} \epsilon_{c}\left(\epsilon_{c}-\epsilon_{u d s}\right)-F_{s} \epsilon_{u d s}\left(\xi R_{b}+1.0\right)-(\xi+1) \lambda R_{b}\left(\epsilon_{b}-\epsilon_{b}^{2}\right)}{\left(\left(\xi R_{b}+1.0\right) F_{s}-R_{c}\left(\epsilon_{c}-\epsilon_{u d s}\right)-\epsilon_{u d s}\right.} \\
R_{b}=\frac{1}{\xi+1} \times \\
\left(\left(\xi R_{b}+1\right) F_{s}-R_{c}\left(\epsilon_{c}-\epsilon_{u d s}\right)-\epsilon_{u d s}\right)^{2} /
\end{gathered}
$$




$$
\begin{gathered}
\left(\xi R_{b}+1\right) F_{d}-(\xi+1.0) \lambda R_{b}\left(\epsilon_{b}-\epsilon_{b}^{2}\right)-\epsilon_{u d s}^{2}-\left(\epsilon_{c}^{2}-\epsilon_{u d s}^{2}\right) R_{c}+ \\
\epsilon_{u d s}^{2} \frac{\xi}{\xi+1.0} R_{b}-\frac{2 \epsilon_{u d s}}{\xi+1.0}\left(\left(\xi R_{b}+1\right) F_{s}-R_{c}\left(\epsilon_{c}-\epsilon_{u d s}\right)-\epsilon_{u d s}\right)
\end{gathered}
$$

The fraction of events by flavor passing the event selection is given in Table 9.2.

\begin{tabular}{|l|c|}
\hline $\begin{array}{l}\text { Event } \\
\text { Flavor }\end{array}$ & $\begin{array}{l}\text { Selection } \\
\text { Efficiency }\end{array}$ \\
\hline$b$ & $(58.3 \pm 0.1) \%$ \\
$u d s c$ & $(57.9 \pm 0.1) \%$ \\
\hline
\end{tabular}

Table 9.2: Event selection efficiencies.

\subsubsection{Statistical Uncertainties with Correlation Effects In- cluded}
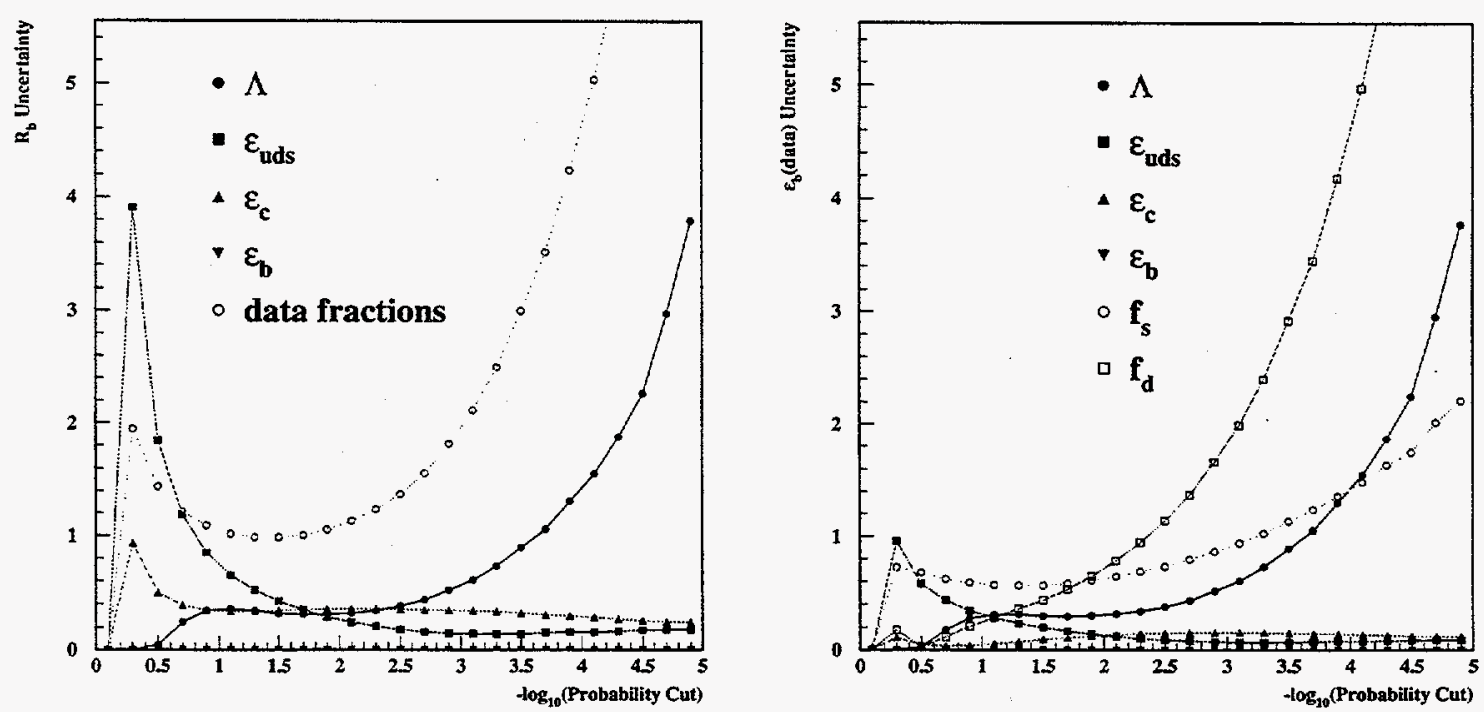

Figure 9.10: Statistical uncertainties in $R_{b}$. Figure 9.11: Statistical uncertainties in $\epsilon_{b}$.

Correlations are carefully handled in the estimation of the statistical uncertainties. The tag counts are separated into three independent categories; the count of events with both hemispheres are tagged $\left(n_{2}\right)$, only one is tagged $\left(n_{1}\right)$, and where neither are 
tagged $\left(n_{0}\right)$. The counts for each case are then varied by their statistical error and the corresponding tag fraction or efficiencies are recalculated. The resulting changes in $R_{b}$ are combined in quadrature to obtain the $R_{b}$ statistical uncertainty. First, one starts by varying a count by its statistical uncertainty:

$$
n_{i}^{\prime}=n_{i} \pm \sqrt{n_{i} \times\left(N_{\text {events }}-n_{i}\right) / N_{\text {events }}}, \quad i=(0,1,2)
$$

The total event count is resummed:

$$
N_{\text {total }}=\sum_{i=0}^{2} n_{i}^{\prime}
$$

Then, the new tagging fractions $\left(F_{s}, F_{d}\right)$ or estimated $b$-tagging efficiencies $\left(\epsilon_{b}, \epsilon_{b}^{\text {double }}\right)$ are recalculated.

$$
\begin{gathered}
F_{s}, \epsilon_{b}=\left(n_{1}^{\prime}+2 \times n_{2}^{\prime} /\left(2 N_{\text {total }}\right)\right. \\
F_{d}, \epsilon_{b}^{\text {double }}=n_{2}^{\prime} / N_{\text {total }}
\end{gathered}
$$

The variation in $R_{b}\left(\delta R_{b}\left(\delta F_{s}^{\delta n_{i}}, \delta F_{d}^{\delta n_{i}}\right)\right)$ and $\epsilon_{b}^{d a t a}\left(\delta \epsilon_{b}^{d a t a}\left(\delta F_{s}^{\delta n_{i}}, \delta F_{d}^{\delta n_{i}}\right)\right)$ from each of these $(i)$ is then summed in quadrature to get the total variation $\left(\delta R_{b}, \delta \epsilon_{b}^{\text {data }}\right)$.

$$
\begin{aligned}
\delta R_{b} & =\sqrt{\sum_{i=0}^{2}\left(\delta R_{b}\left(\delta F_{s}^{\delta n_{i}}, \delta F_{d}^{\delta n_{i}}\right)\right)^{2}} \\
\delta \epsilon_{b}^{d a t a} & =\sqrt{\sum_{i=0}^{2}\left(\delta \epsilon_{b}^{d a t a}\left(\delta F_{s}^{\delta n_{i}}, \delta F_{d}^{\delta n_{i}}\right)\right)^{2}}
\end{aligned}
$$

The statistical uncertainties on $R_{b}$ and $\epsilon_{b}^{\text {data }}$ are shown in Fig. 9.10 and Fig. 9.11 versus the hemisphere probability cut.

\subsection{Measurement Results}

The $b$-tagging purity $\left(\Pi_{b}\right)$ versus $\epsilon_{b}$ performance of the $b$-tag is shown in Fig. 9.12. Also shown in this figure is the ALEPH hemisphere tag performance[50]. The $b$-tag performance for the hemispheres versus $\Pi_{h e m i}$ is shown in Fig. 9.13. Note that the estimated and measured $b$-tagging efficiencies agree well over the whole probability range. Deviations between these values would indicate either incorrect physics simulation or detector resolution simulation. These efficiencies along with the $b$-hemisphere correlations measured from the Monte Carlo and the single and double hemisphere tagging fractions in data collected from the 1993 and 1994 SLD runs yield the $R_{b}$ values shown in Fig. 9.14. The results have been corrected for the event selection bias. 

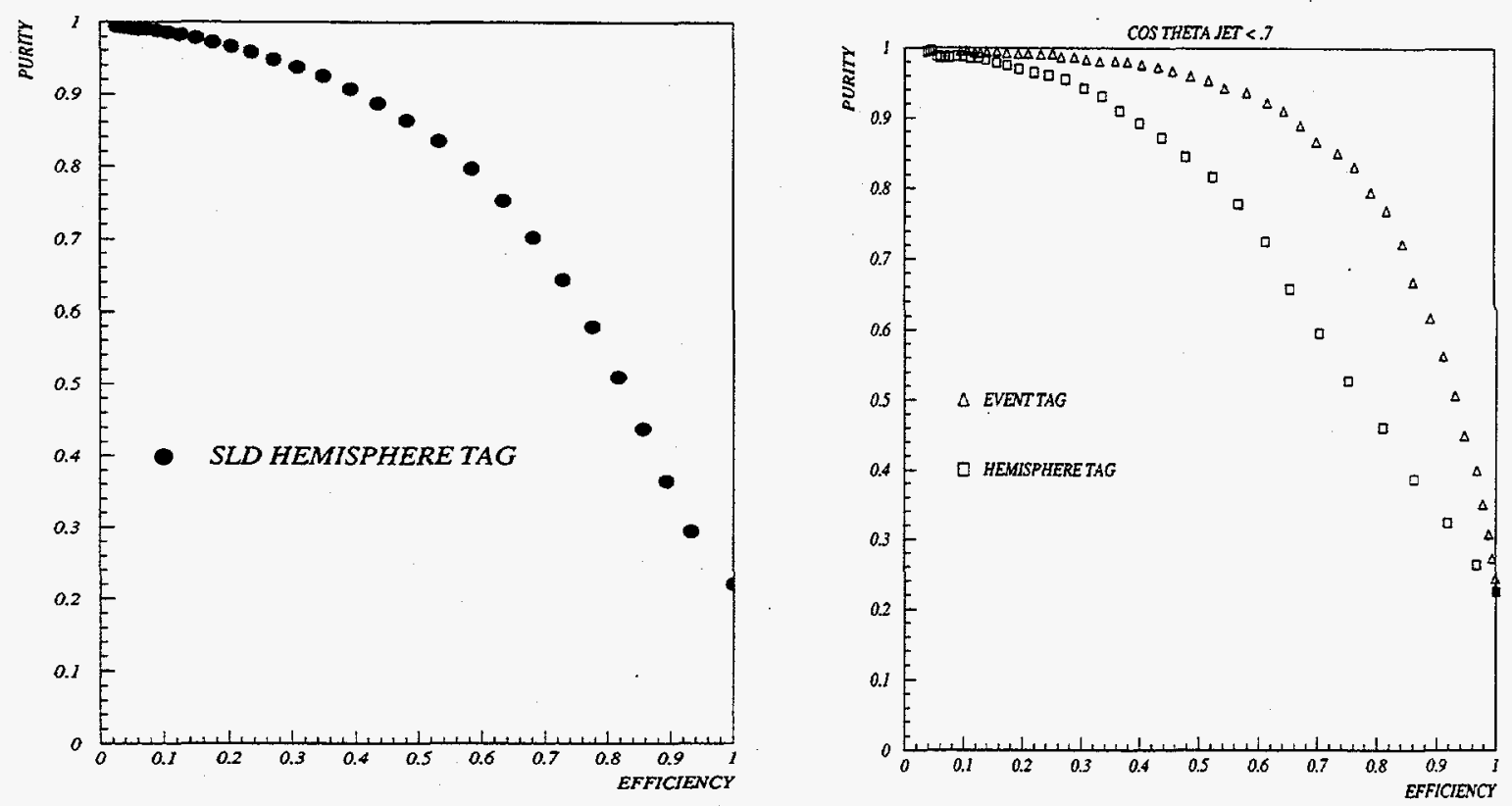

Figure 9.12: Tag performance comparison between SLD and ALEPH.

\subsection{Systematics}

The systematics come from correlation uncertainties, $c$-tagging efficiency uncertainties, and uds tagging efficiency uncertainties. The same weighting procedures as described for the event tag were used with correlations being taken into account. These systematics are described below:

\subsubsection{Physics Systematics}

\section{- Correlation Systematics}

Contrary to the event tag method the $b$-physics systematics enter only indirectly through the correlation estimation since the $b$-tagging efficiency is simultaneously measured from the data. The same variations in the physics parameters, as described for the event tag, were used in estimating the contributions here. The results are shown in Fig. 9.15. 


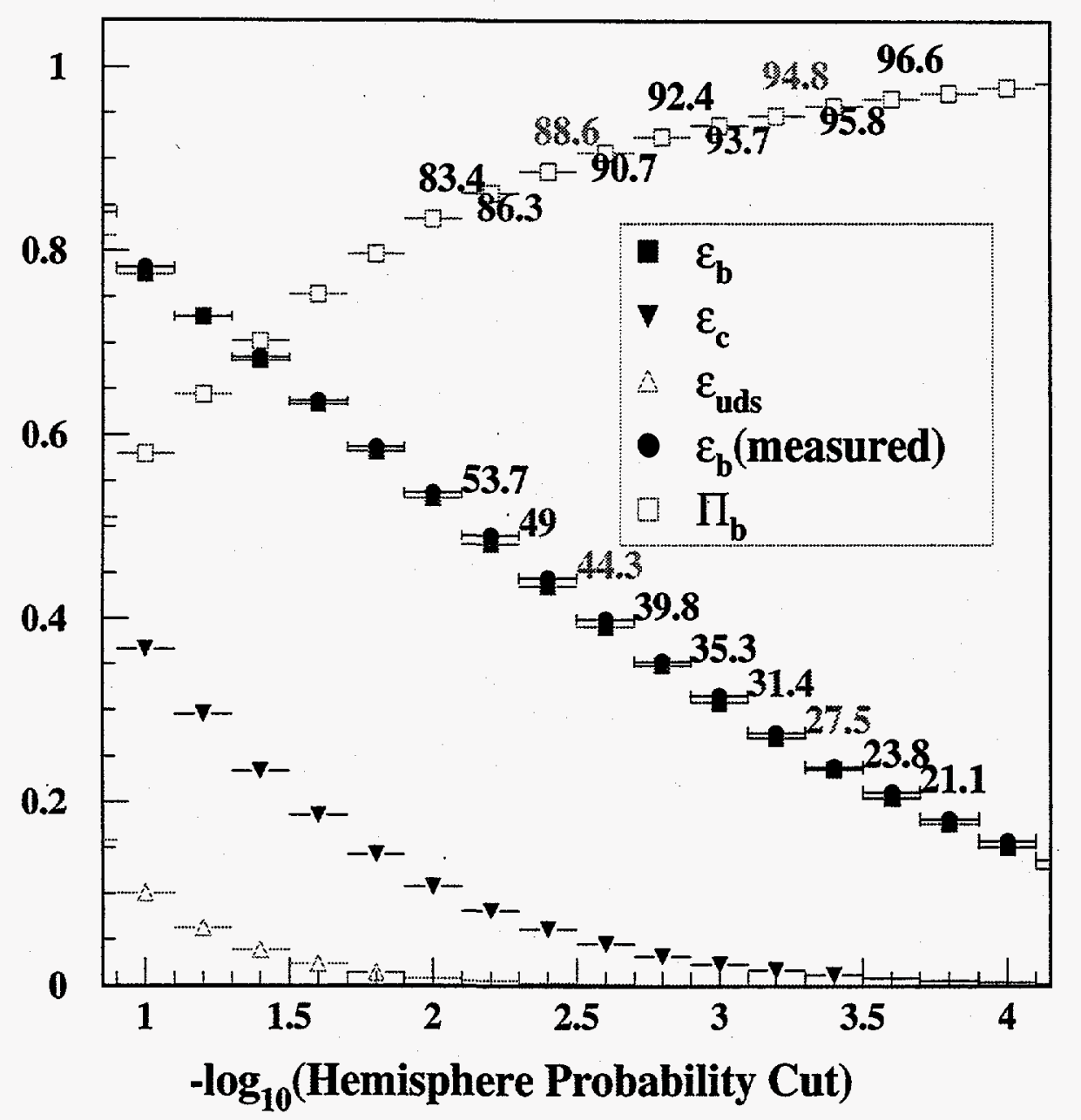

Figure 9.13: $b$-tag dependence on hemisphere probability cut.

- Charm systematics

Again, the same variation in the physics modelling parameters as for the event tag were used. As is shown in Fig. 9.15, the largest contribution to the total uncertainty comes from the estimation of the charm tagging efficiency. This is expected as it has the largest tagging efficiency not measured from the data. The largest contribution is from the uncertainty in the charm fragmentation. 


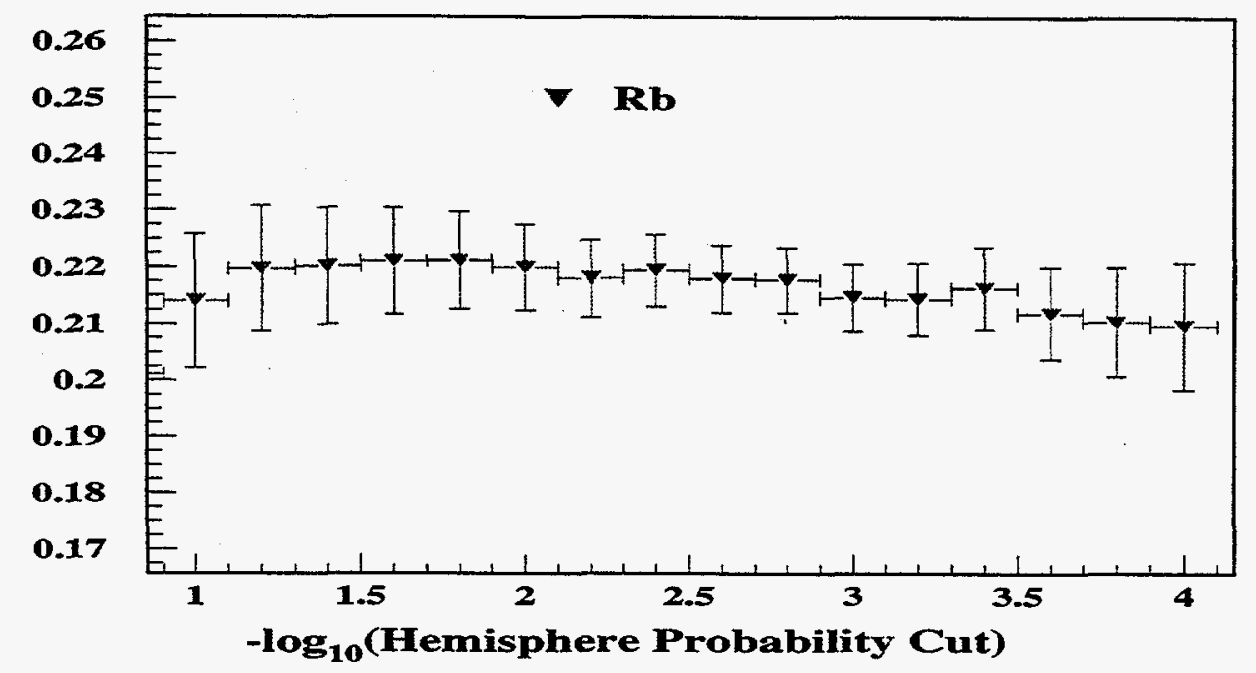

Figure 9.14: $R_{b}$ from the lifetime probability tag versus the hemisphere probability cut.

Comparable in magnitude to this is the rate at which $D^{+} \mathrm{s}$ are produced in $c$ events relative to other charmed hadrons. As expected, the $D^{+}$contribution dominates over the others due to is significantly longer lifetime.

- $R_{c}$

There is a correlation of $R_{b}$ with $R_{c}$ of -0.14 . The current uncertainty on $R_{c}$ of $10 \%$ gives the systematic from $R_{c}$ shown in Fig. 9.15.

\section{- Light Quark Systematics}

As the efficiency for tagging light quarks is very small relative to $\epsilon_{c}$ the light quark systematic contributions are likewise small despite the factor of 3 greater production. As is shown in Fig. 9.15 all of the systematics are well under $1 \%$. Due to concern about the lack of knowledge about gluon splitting in light quark events and the potential sensitivity that this measurement might have to it the amount has been varied by $100 \%$ compared to the $50 \%$ used for the event tag. 


\subsubsection{Detector Systematics}

The detector systematics include tracking efficiency and resolution corrections. The affects of these on $R_{b}$ and $\epsilon_{c}$ are shown in Fig. 9.16 as a function of the hemisphere probability cut. Other contributions coming from tails on the primary vertex position
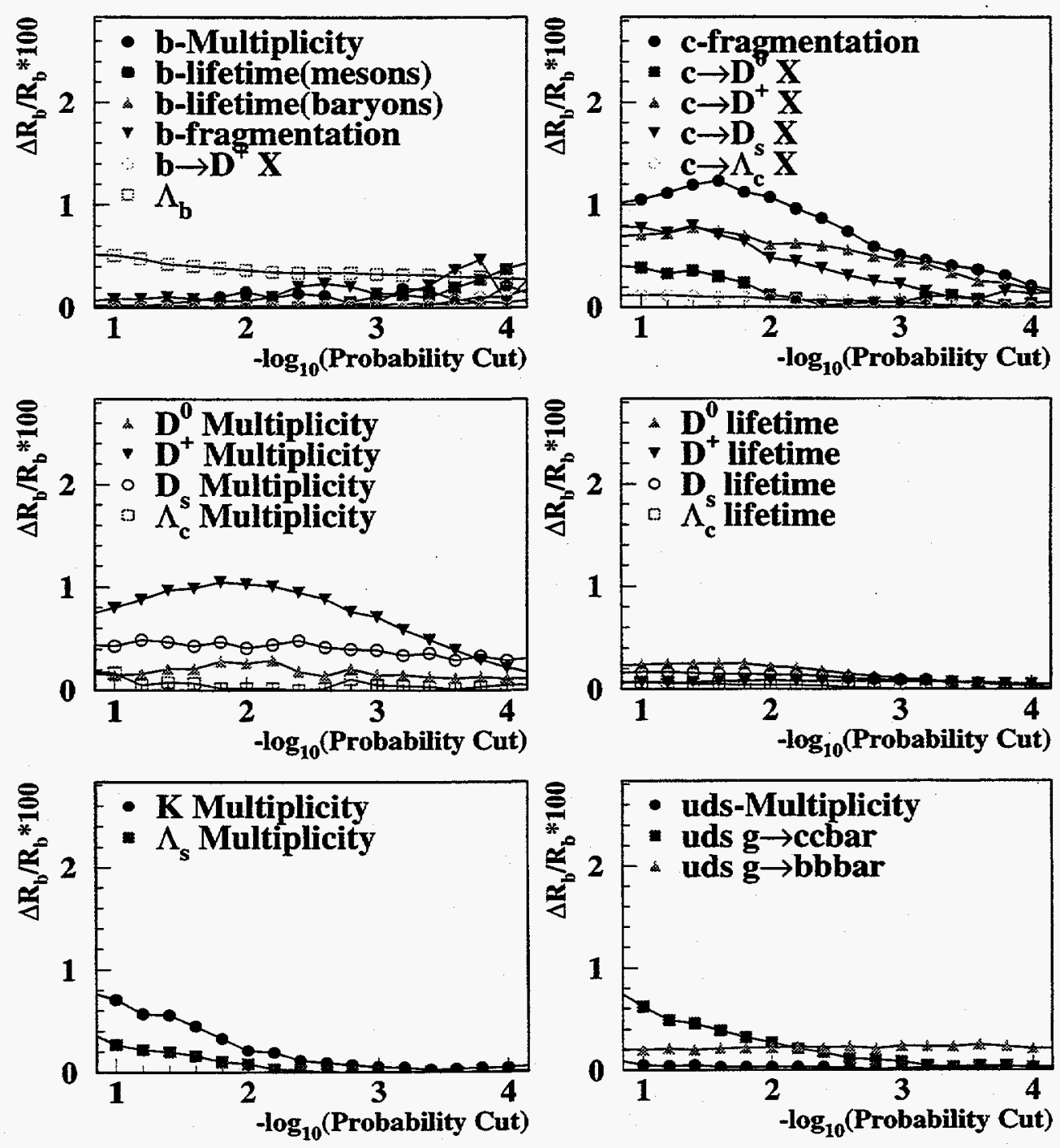

Figure 9.15: Physics contributions to the uncertainty in $R_{b}$ versus the hemisphere probability cut. 
measurement and from $V^{0}$ rejection are not included in this plot and are only given at the optimal probability cut.
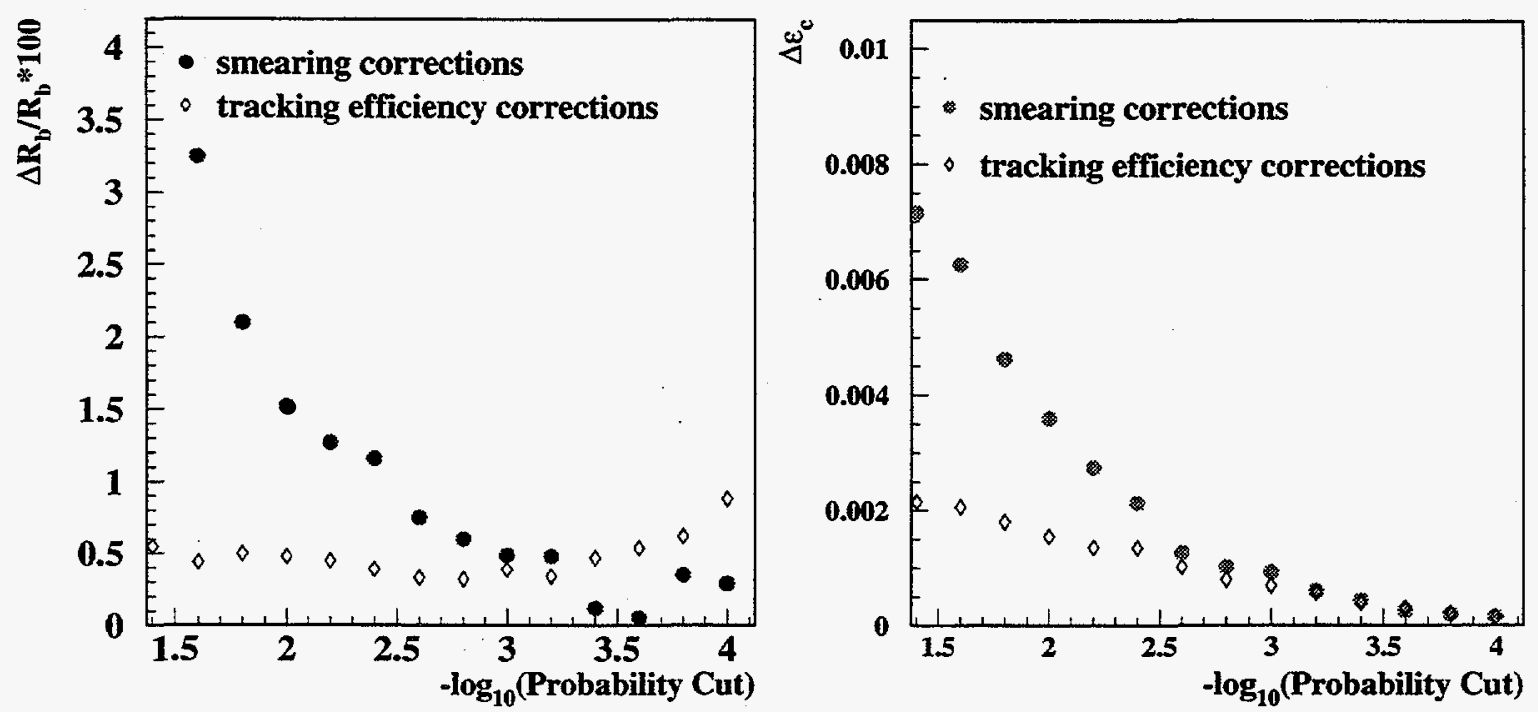

Figure 9.16: Uncertainties in $R_{b}$ from effi- Figure 9.17: Uncertainties in $\epsilon_{c}$ from efficiency and smearing corrections. ciency and smearing corrections.

\section{- Impact Resolution Systematics}

The full amount of the change caused by the smearing corrections described in Sect. 9.3.1 is taken as the systematic. In Fig. 9.17 this uncertainty is shown as strongly decreasing with tighter cuts. This is due directly to the decrease in $\epsilon_{c}$ with tighter cuts.

\section{- Tracking Efficiency Corrections}

The same variations as described in Sect. 7.3 are used to determine the systematic from the efficiency corrections. The resulting variations in $R_{b}$ are summed in quadrature. The dominant contribution comes from the 0.4 track per event variation. The contribution from each variation as a function of the probability cut is show in Fig. 9.18. The probability tag is much less sensitive, than the event tag, to these uncertainties. This is understood as $\epsilon_{b}$ is not affected since it is measured from the data and the uncertainty mostly enters just through 


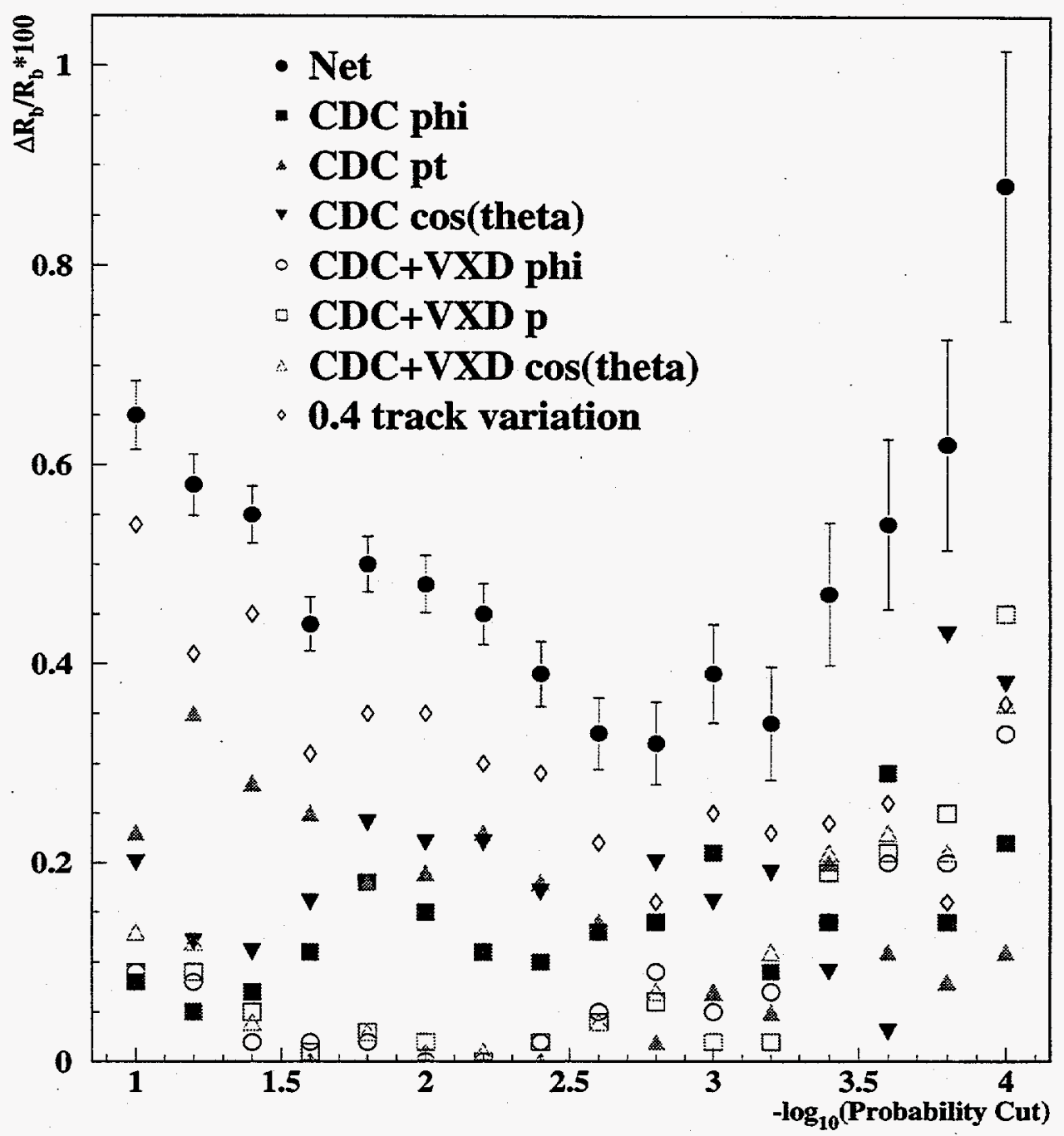

Figure 9.18: Uncertainties in $R_{b}$ from efficiency corrections.

$\epsilon_{c}$. The uncertainty in $\epsilon_{c}$ caused by the efficiency uncertainties is shown in Fig. 9.17 .

- Primary Vertex Position

The measurement was repeated with simulation of the primary vertex position tails described in Sect. 4 added. At the optimal cut of $10^{-2.8}$ the resulting variation is $0.3 \%$ and is taken as the systematic. 


\section{- $V^{0}$ rejection}

The measurement was reperformed with the $V^{0}$ rejection removed. At the optimal cut of $10^{-2.8}$ the total change was $0.9 \%$. Since, this is a significant over estimate of the systematic, half of this change is used.

A general feature of all of the physics and smearing systematics is that they decrease with tighter tagging requirements. What prevents one from using a very tight cut are the contributions from the statistical uncertainties. This is promising as it implies that with more data both statistical and systematical uncertainties will decrease as one can move towards tighter cuts. An optimal set of cuts having the highest combined (statistical plus systematic) precision can always be found.

\subsubsection{Verifications}

The result is checked for sensitivity to cuts, consistency between inputs from the individual runs used, and any geometrical effects. Furthermore, attempts at independently determining the contributions to the correlation systematics are made.

\subsubsection{Sensitivity to Cuts}

Variations in the $R_{b}$ result versus the probability cut would indicate simulation problems. Deviations from the central at low probability cuts would suggest a problem with the resolution simulation. Deviations at high probability cuts would suggest problems with the tracking efficiency simulation. As can be seen from Fig. 9.14 no deviations outside of the statistical plus systematic error bars are observed.

\subsubsection{Tag Inputs versus Position}

The significance variable $\chi$ is checked for any unsimulated variations as function of position within the detector. Such variations can cause contributions to the correlation. For example, in symmetric inefficiencies the jets from both quarks may have tracks in inefficient regions causing a positive correlation contribution. As can be seen from Fig. 9.19 and Fig. 9.20 no aberrations are observed. 

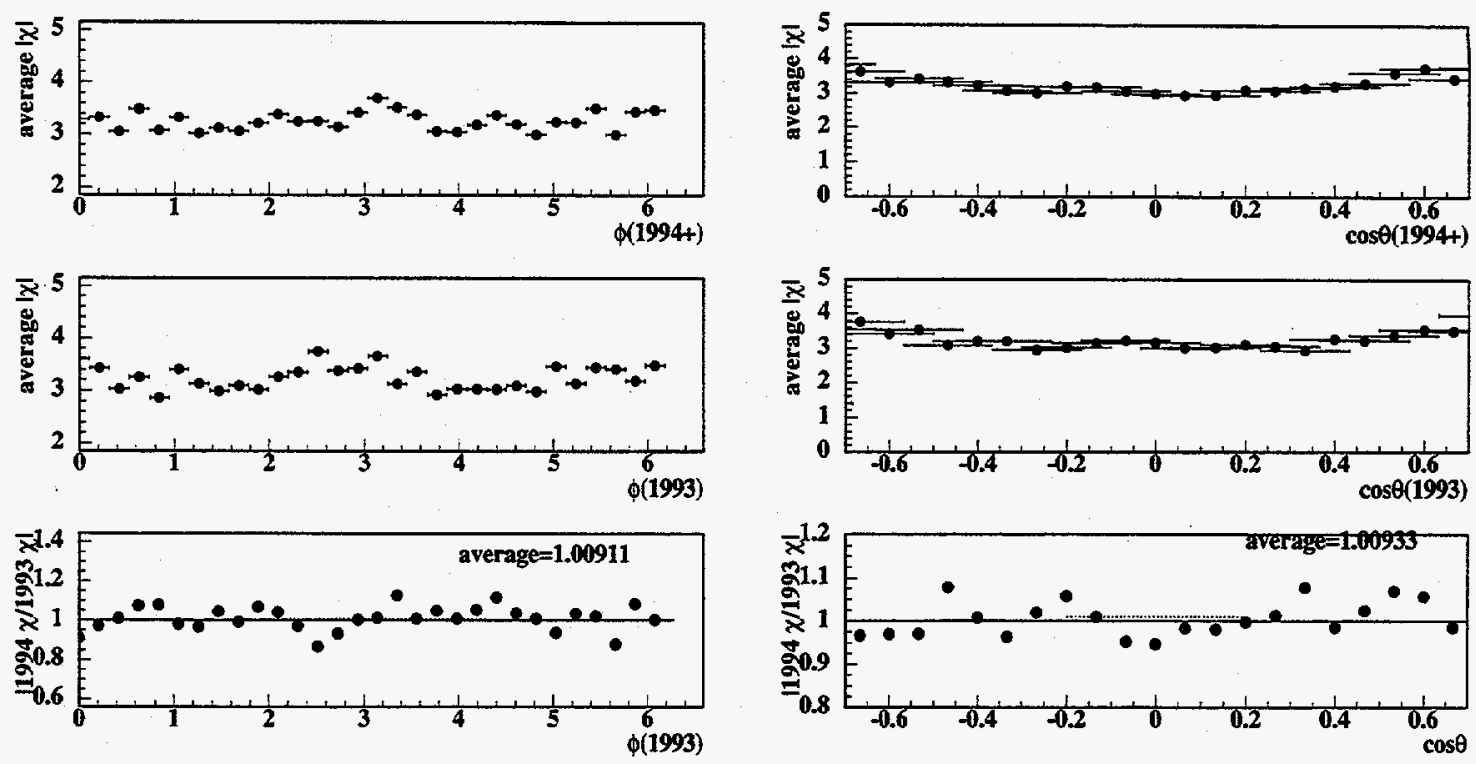

Figure 9.19: Distribution of the significance Figure 9.20: Distribution of the significance variable $\chi$ versus the azimuthal angle of the variable $\chi$ versus the polar angle of the dedetector. tector.

\subsubsection{Run Dependant Variations}

It is important to check for run periods where either bad beam positions or detector failures may have caused errantly high tagging rates that would not be simulated properly. In Fig. 9.21 the average $|\chi|$ versus run number for 1993 and 1994 is shown. No significant flyers are revealed.

\subsubsection{Correlation Components}

The correlation between the $b$-hemispheres results from both physics and detector effects. The correlation between the $b$-hemispheres is shown versus the hemisphere probability cut in Fig. 9.22. It is desirable to isolate the contributions to the correlation to ensure that all systematic contributions have been accounted for and not duplicated. A method has been developed whereby components of the correlation may be projected out[8]. The method involves determining how much of an influence tagging in one hemisphere as a function of some variable $\nu$ has on the distribution 

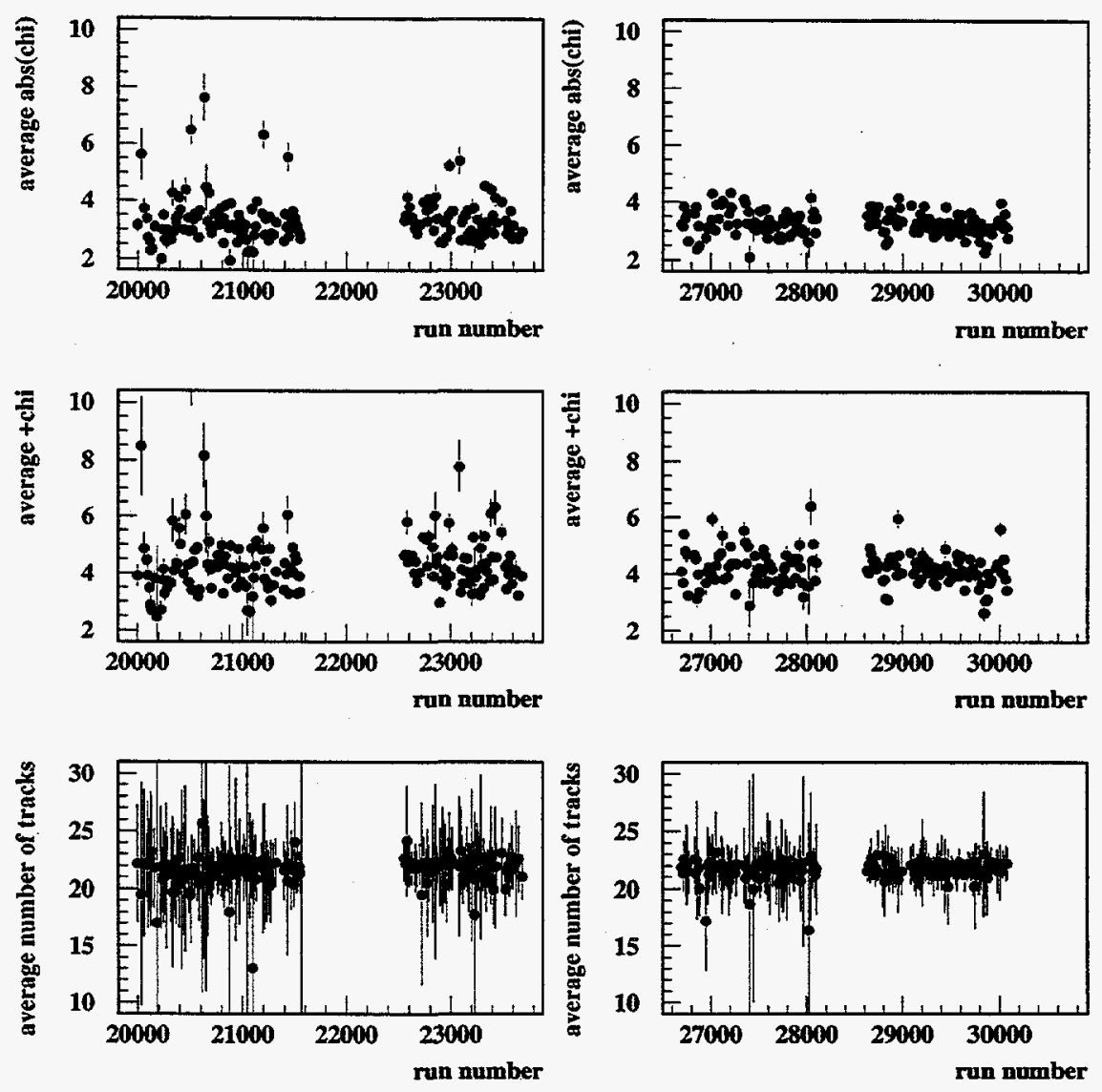

Figure 9.21: Average $\chi$ and track counts for the 1993 and 1994 runs.

of $\nu$ in the other hemisphere. To calculate the correlation the distribution of the efficiency as a function of $\nu$ is convoluted with the distribution of $\nu, h(\nu)$ in a hemisphere after the other hemisphere has been tagged as shown in Eqn. 9.7. Effectively, the correlation component is a measure of the probability that if a hemisphere has a value $\nu$ that is in a high(low) tagging efficiency region the other hemisphere is also in a high(low) tagging efficiency region.

$$
\epsilon_{\nu}=\int \epsilon(\nu) h(\nu) d \nu
$$


The influenced distribution $h(\nu)$ is determined from the convolution of the histogram $\left(H\left(\nu_{1}, \nu_{2}\right)\right)$ containing $b$ event counts versus $\nu_{h e m i 1}$ and $\nu_{h e m i 2}$ with the efficiency, $\epsilon_{\nu}$

$$
h(\nu)=\sum_{\nu_{1}} H\left(\nu_{1}, \nu_{2}\right) \cdot \epsilon\left(\nu_{1}\right)
$$

The double tag efficiency is then $\epsilon \times \epsilon_{\nu}$ thus the correlation component is:

$$
\lambda_{\nu}=\frac{\epsilon \cdot \epsilon_{\nu}-\epsilon^{2}}{\epsilon-\epsilon^{2}}
$$

Ideally, if all of the component have been identified then the sum of all of the components will add to the total correlation. It is necessary to insure that the components are independent. The components that have been studied are the $\cos \theta$ of the quark direction, the $\phi$ of the quark direction, the momentum of the quark, the deviation of the $r \phi$ and $r z$ of the beam position used from the true beam position, the $B$-lifetime and the $b$-momentum sum.

In Fig. 9.23 the distributions used to calculate a component of the correlation are shown. In this case $\nu$ is $\cos \theta$. The top left plot shows the distribution of $\nu$ over the full range of the variable. Next, is shown the $b$-hemisphere tagging efficiency versus $\nu$. The distribution of $\nu$ (the line) for tagged hemispheres compared to the distribution $h(\nu)$ obtained after the other hemisphere has been tagged is shown. If no correlation between the hemispheres existed then the distributions would be identical. In this case the convolution of $\epsilon(\nu)$ and $h(\nu)$ would yield the b-hemisphere tagging efficiency, $\epsilon$. As can be seen from equation 9.9 this would result in $\lambda_{\nu}=0$.. Finally the ratio of the influenced distribution to the original distribution is shown to clarify the $\nu$ dependence of the correlation. The distributions are for a cut of $\Pi_{\text {hemi }}=10^{-2.0}$ which was chosen because the correlation components are large at this cut.

\section{- $\cos \theta$ component}

Correlations in $\cos \theta$ are expected due to the back to back nature of the quark jets. If one jet points in a direction of low tagging efficiency so should the other thus a positive correlation should be observed. 


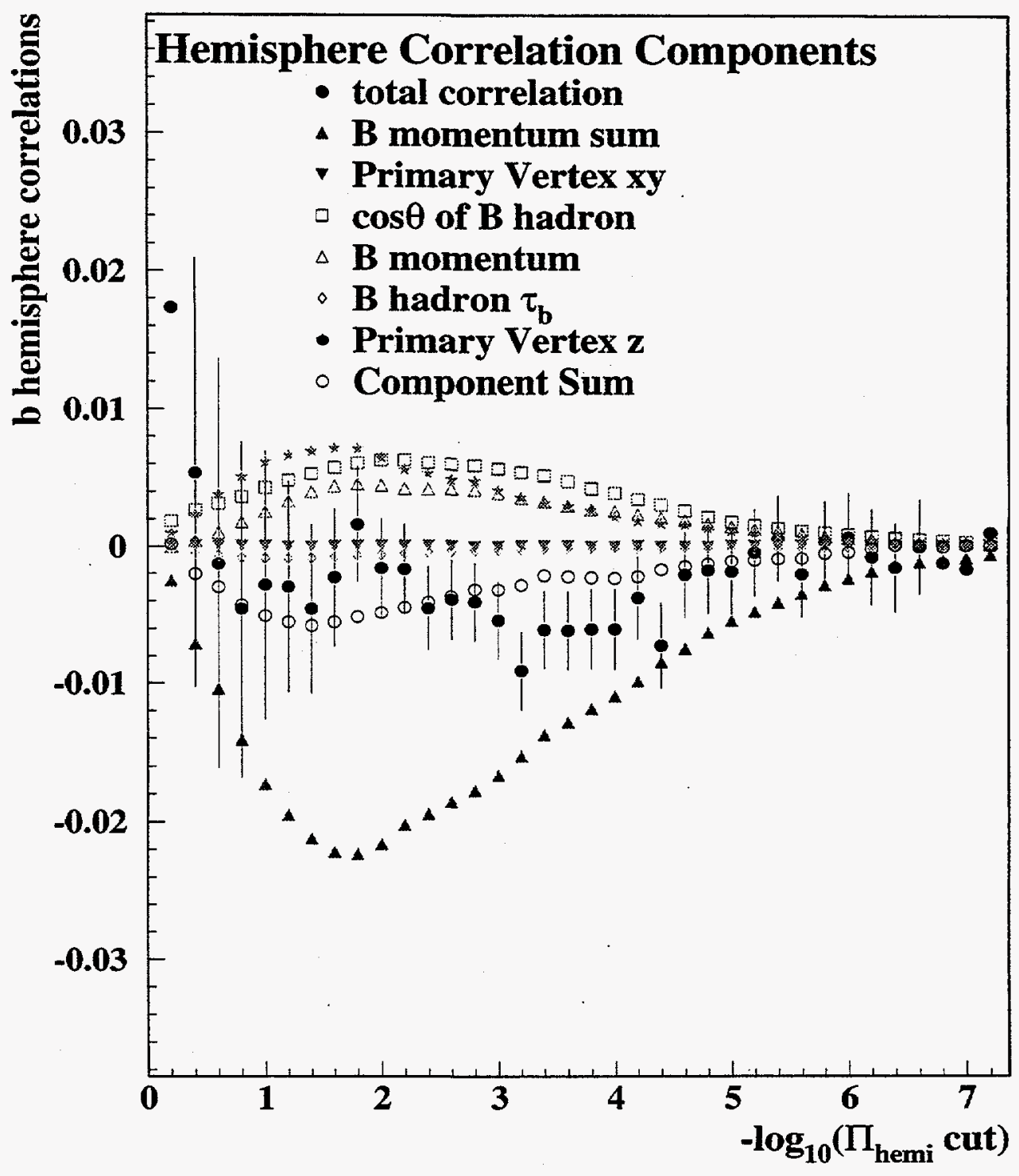

Figure 9.22: $b$-hemisphere correlation components.

- $\phi$ component

No correlation in $\phi$ is expected as the detector is nearly axially uniform. It is both a check for whether an error is causing a correlation to be seen where there isn't one and any unexpected detector defects. No correlation in the $\phi$ component is observed.

- components from poorly measured beam positions 


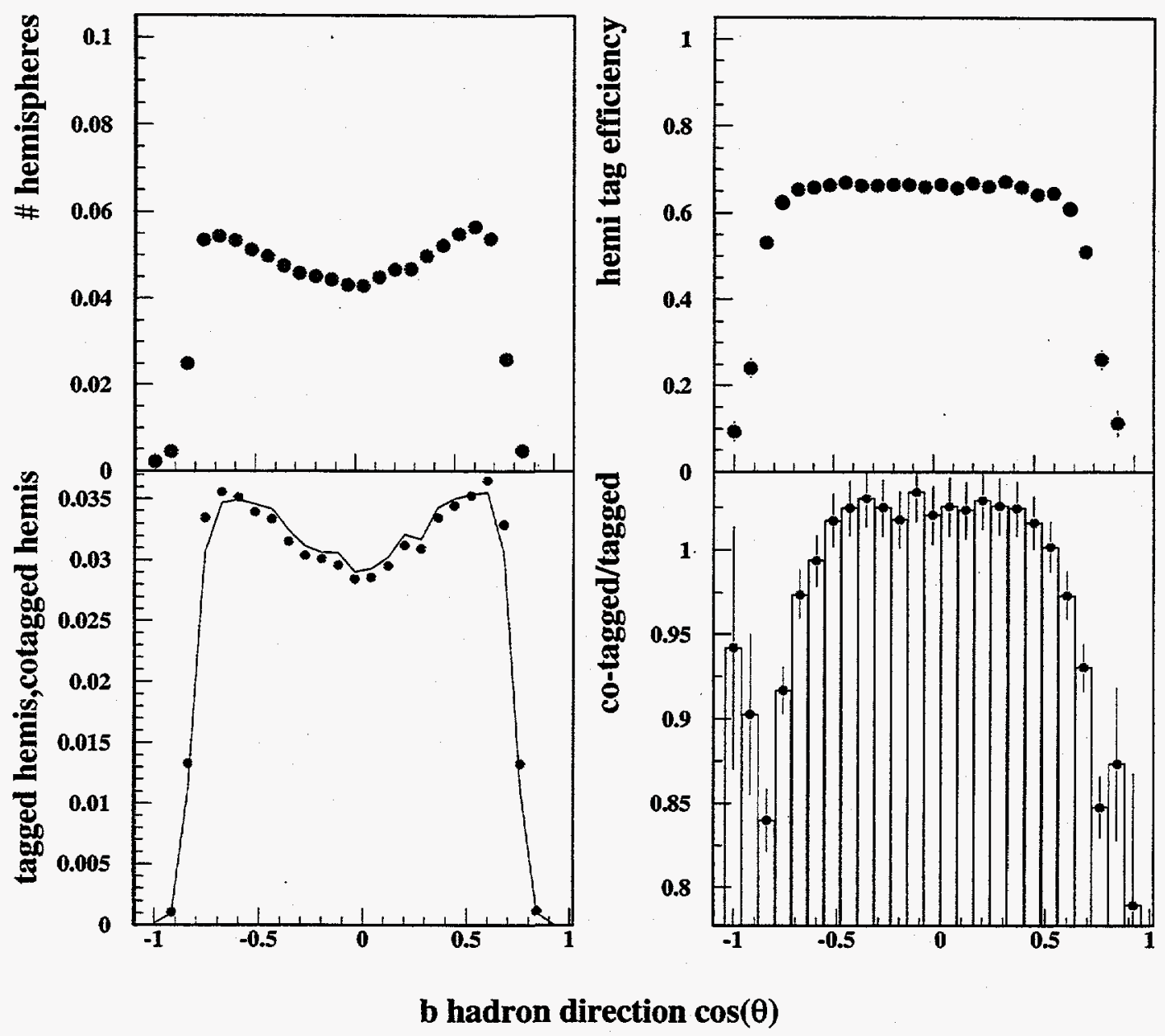

Figure 9.23: Distributions used to determine component of the correlation from the polar angles of the $B$-hadrons.

Poor beam position measurements can lead to both quarks going into the same hemisphere which would give a negative correlation and can also cause the probability for tracks in both hemisphere to be consistent with the primary vertex to be low thus causing both to tag and a positive correlation. The latter is clearly seen for the dependence on the longitudinal position shown in Fig. 9.24 . 


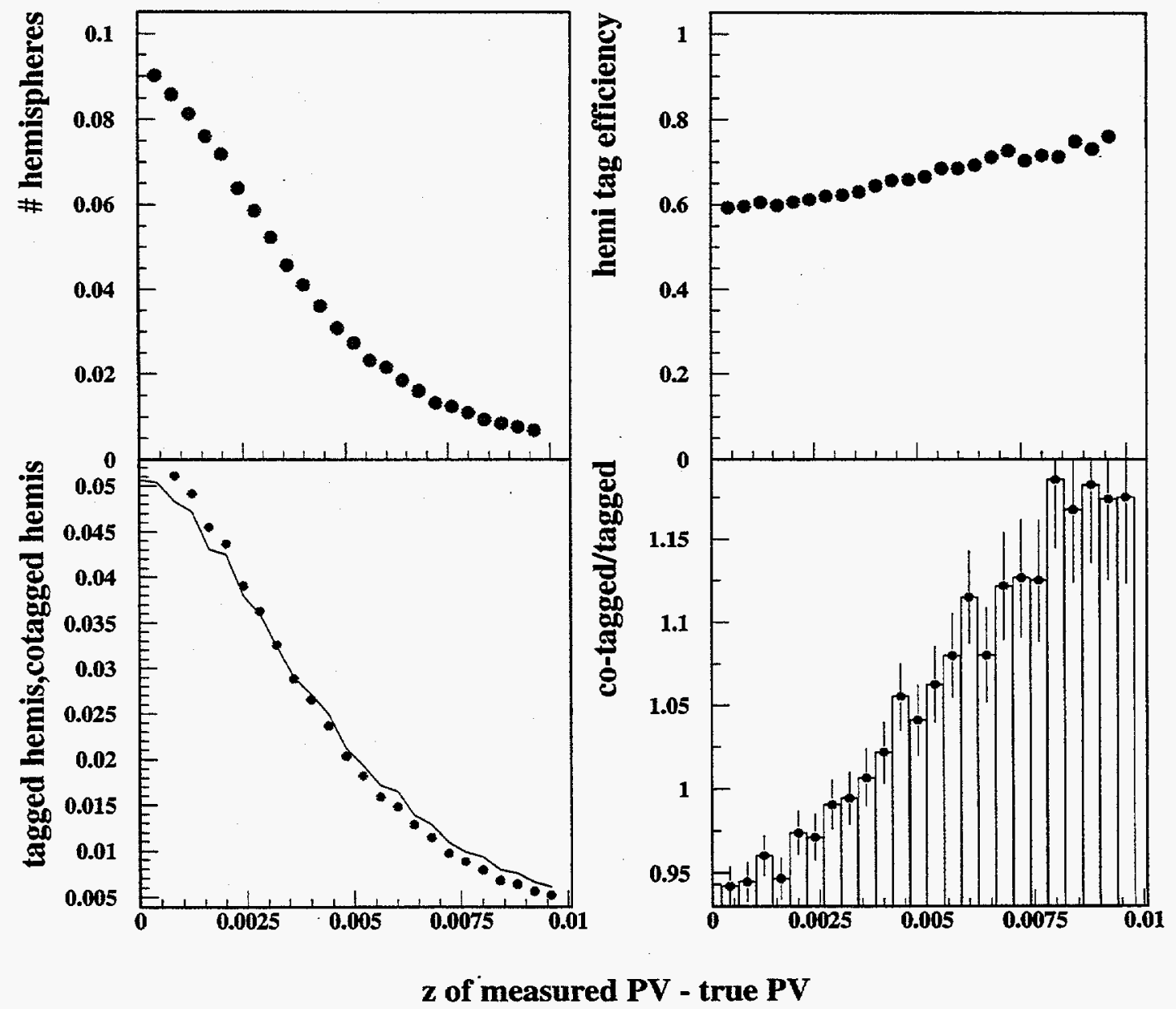

Figure 9.24: Distributions used to determine primary vertex $z$ correlation component.

- B-lifetime component

Theoretically, there is not an obvious means by which the lifetime of the hadrons in the separate hemispheres may communicate with each other except through erroneous beam positions. However, a small negative correlation is observed even in the case where the true Monte Carlo primary vertex position has been used. This is continuing to be investigated. At the cut for which the result is given, this correlation is negligible. 


\section{- $B$-momentum sum}

The $B$-momentum sum variable is designed to account for gluon activity in the event. The variable is chosen to be:

$$
\nu=\frac{\left(\vec{P}_{B}+\vec{P}_{\bar{B}}\right) \cdot \hat{H}}{\left|\vec{P}_{B}+\vec{P}_{\bar{B}}\right|}
$$

where $\hat{H}$ is the axis for the corresponding hemisphere. In the case where both $B$ 's go into the same hemisphere the value of $|\nu|$ will be large and the hemisphere for which it is positive should have a high probability of tagging while the other has a negligible chance of being tagged. The result is a negative correlation between the hemispheres.

\section{- B-momentum}

The $B$-momentum measures correlations from the fragmentation process. Here, the variable $\nu$ is the momentum of the $B$-hadron for each hemisphere when they have gone into separate hemispheres.

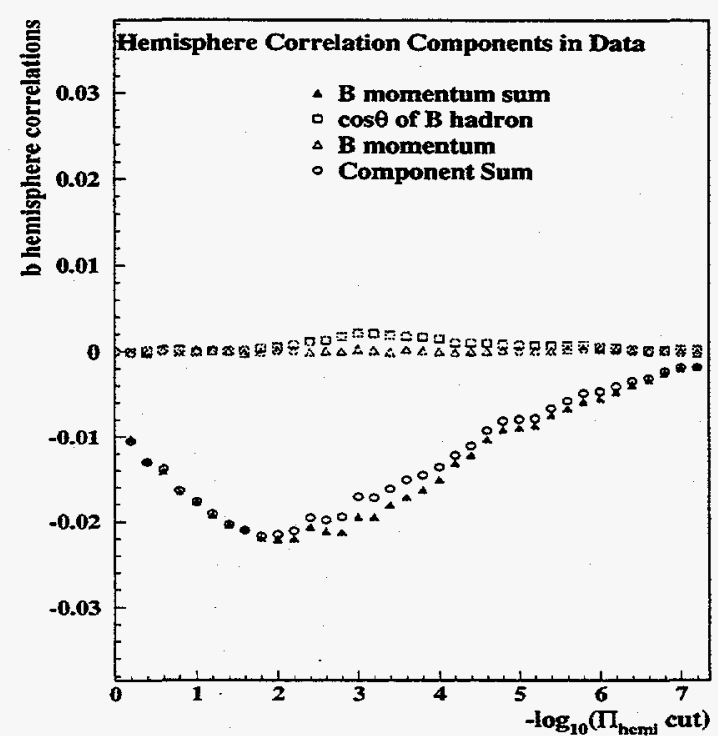

Figure 9.25: Correlation components estimated from the data.

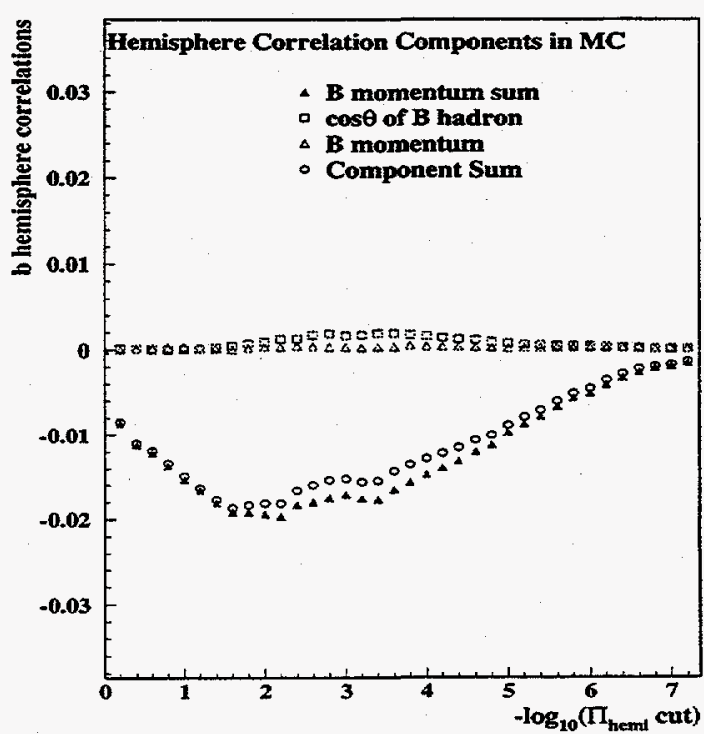

Figure 9.26: Correlation components estimated from the Monte Carlo. 
The total correlation along with its components are shown in Fig. 9.22. The majority of the components show a positive correlation between the hemispheres. These are balanced by the large negative component from the gluon activity represented by the $B$-momentum sum. The behavior of the curves versus probability cut is expected. At very loose cuts all hemispheres are being tagged and, of course, no correlation can exist. At tight cuts the $b$-hadrons are likely to have most of the beam energy and to have decay far from the measured primary vertex position. Thus, the influence from gluons(which is reflected in the energy of the $b$ 's) and the influence of the $b$-lifetime and primary vertex position errors(which are relative to the displacement of the $b$ from the primary vertex) clearly become negligible. A $b$ that is tagged with very low probability is also likely to have decayed in the direction of a high precision high efficiency region of the detector where dependence on the polar angle is negligible.

The total correlation and the sum of the correlation components are consistent within errors. Thus, there is no evidence that any large unexpected systematics entering through the correlation exist. These tests have been performed using only the Monte Carlo. A check of the correlation simulation was performed as described in the following section.

\subsubsection{Check of correlation components from data}

An approximation of the correlation components can be obtained from the data. The truth information for the $B$-hadron directions has been replaced with the directions of the highest momentum jets and the momentums also come from these jets. With this replacement the correlations can be determined from the data and may be compared to those obtained from a properly flavor mixed Monte Carlo sample. To reduce the contamination from light quark events an event tag similar to the 2-D impact tag but with the 2-D impact parameter replaced with the $\chi$ variable and with cuts of at least 3 tracks with $\chi>3.0$ is used. The $b$-efficiency for this tag is $72 \%$ and the $b$ purity is $85 \%$. The resulting correlation components are shown in figures 9.25 and 9.26. Certain correlations such as the displacement of the measured from the true primary vertex positions could not be approximated. Also, the $\cos \theta$ correlation is 
reduced by performing the event tag. However, the expected features are seen. The $\cos \theta$ component is positive and larger than the $B$-momentum component and the gluon activity from the $B$-momentum sum appears to be the largest contributor to the correlation.

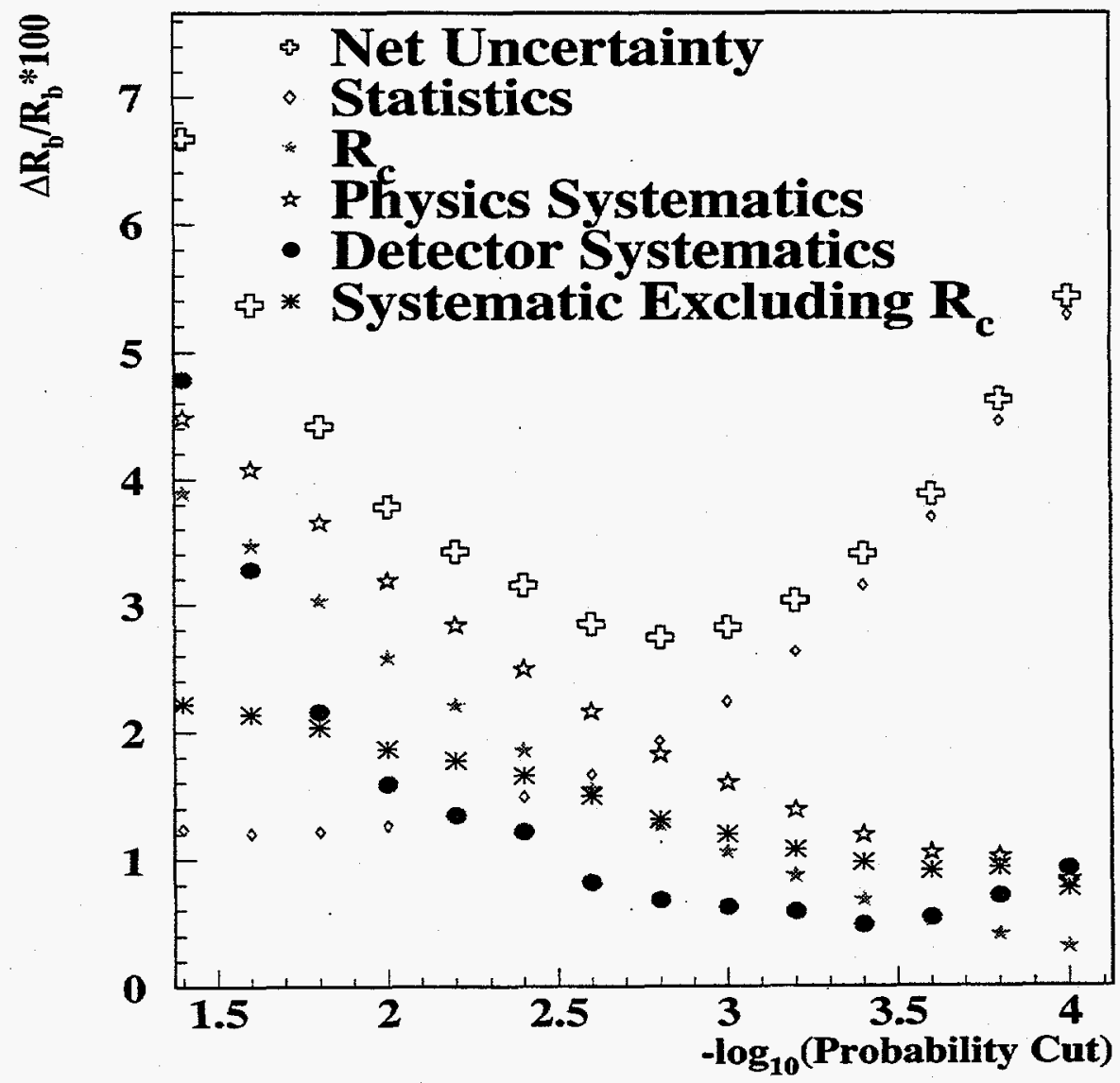

Figure 9.27: Contributions to the uncertainty in $R_{b}$ versus the hemisphere probability cut.

The Monte Carlo appears to underestimate the gluon activity by $-3 \%$. Increasing the true $B$-momentum sum correlation by $3 \%$ causes a change in the correlation sum of $1.6 \times 10^{-4}$. The change in $R_{b}$ from this variation in the correlation is $0.3 \%$. 


\subsubsection{Summary of Systematics}

The curves showing all detector, physics and $R_{c}$ systematic and statistical uncertainties are displayed in Fig. 9.27. The contributions from the systematics on $\epsilon_{c}$ cause greater uncertainty at looser tag cuts while the statistical uncertainties start to dominate for tighter cuts. The minimum combined systematic occurs at $-\log _{10} \Pi_{h e m i}=10^{-2.8}$. At this cut the combined uncertainty from all sources is $\delta R_{b}=2.8 \%$.

\subsection{Final Result}

The measurement of $R_{b}$ is made at the point which minimizes the combined statistical plus systematic uncertainties. This occurs at the cut of $P_{c u t}=10^{-2.8}$. After applying all efficiency and resolution corrections and correcting for biases introduced by the event selection, the results shown in Tables $9.3,9.4$ and 9.5 are obtained with the final result for $R_{b}$ from the probability tag being

$$
R_{b}=0.218 \pm 0.004_{\text {statistical }} \pm 0.004_{\text {systematic }} \pm 0.003_{R_{c}}
$$

\begin{tabular}{|l|c|}
\hline \multicolumn{2}{|c|}{ Monte Carlo Measurements } \\
\hline$\epsilon_{b}$ & $34.8 \%$ \\
$\Pi_{b}$ & $92.4 \%$ \\
$b$ hemisphere correlation & $-0.53 \%$ \\
\hline
\end{tabular}

\begin{tabular}{|c|c|}
\hline \multicolumn{2}{|c|}{ Data Measurements } \\
\hline$\epsilon_{b}$ & $(35.3 \pm 0.007) \%$ \\
$F_{s}$ & $8.4 \%$ \\
$F_{d}$ & $2.8 \%$ \\
\hline
\end{tabular}

Table 9.3: Probability tag performance for $P_{\text {cut }}=10^{-2.8}$. 


\begin{tabular}{|l|c||c|c|}
\hline \multicolumn{4}{|c|}{ Correlation Systematics } \\
\hline Systematic & $\delta R_{b} / R_{b}$ & Systematic & $\delta R_{b} / R_{b}$ \\
\hline$\tau_{B}$ & $0.1 \%$ & $B$-decay multiplicity & $0.1 \%$ \\
$B \rightarrow D$ & $0.1 \%$ & $b$ fragmentation & $0.2 \%$ \\
$\Lambda_{b}$ fraction & $0.3 \%$ & \\
\hline \multicolumn{4}{|c|}{ Charm Systematics } \\
\hline Systematic & $\delta R_{b} / R_{b}$ & Systematic & $\delta R_{b} / R_{b}$ \\
\hline$c \rightarrow D$ & $0.6 \%$ & $c$ fragmentation & $0.6 \%$ \\
$D^{0}, D^{ \pm}, D_{s}, \Lambda_{c}$ production & $0.9 \%$ & $D^{0}, D^{ \pm}, D_{s}, \Lambda_{c}$ lifetime & $0.2 \%$ \\
$R_{c}$ & $1.3 \%$ & \\
\hline \multicolumn{4}{|c|}{ Light Quark Systematics } \\
\hline Systematic & $\delta R_{b} / R_{b}$ & Systematic & $\delta R_{b} / R_{b}$ \\
\hline$K, \Lambda_{s}$ production & $0.1 \%$ & $g \rightarrow c \bar{c}, b \bar{b}$ & $0.2 \%$ \\
\hline
\end{tabular}

Table 9.4: Summary of physics systematics for probability tag at a cut of $P_{\text {cut }}=10^{-2.8}$

\begin{tabular}{|l|c|l|c|}
\hline \multicolumn{4}{|c|}{ Detector Systematics } \\
\hline Systematic & $\delta R_{b} / R_{b}$ & Systematic & $\delta R_{b} / R_{b}$ \\
\hline Efficiency Corrections & $0.5 \%$ & $V^{0}$ Rejection & $0.5 \%$ \\
Impact Resolutions & $0.5 \%$ & Beam Position Tails & $0.3 \%$ \\
\hline
\end{tabular}

Table 9.5: Summary of detector systematics for probability tag at a cut of $P_{\text {cut }}=10^{-2.8}$ 


\section{Chapter 10}

\section{Implications and Future Prospects}

The combined result from this thesis is $R_{b}=0.220 \pm 0.005$. The event tag method yields a transparent measure of $R_{b}$ unaffected by the complications of determining hemisphere correlations while providing the tools for the hemisphere tag. The hemisphere tag has yielded a competitive measurement that will improve both statistically and systematically with data from future SLD runs. With the results from the 3-D impact parameter tag and 3-D displaced vertex tag (see Fig. 10.1 and Appendix B) included, the result remains $R_{b}=0.220 \pm 0.005$. The correlation between the event tag results has been included using the method of Lyons, Gibbaut and Clifford[59]. Only the ALEPH and DELPHI microvertex tag methods currently exceed this measurement in accuracy. They benefit from their large data samples. While the goal of making a $1 \%$ measurement has not been achieved due to a limited data sample size, the potential for achieving the desired precision has been demonstrated. The systematics are themselves a function of the number of available data events. With more data, the statistical contribution at high probability cuts will and the cut may be tightened. This will result in reduced contamination of the tag from $c$ and $u d s$ events thus leading to a corresponding lower systematic contribution from these. This is shown in the Fig. 9.27.

The result for $R_{b}$ is $1 \sigma$ high compared to the Standard Model prediction. To achieve a precision of $1 \%$ a probability cut of at least $10^{-4}$ must be used to reduce the systematics. Roughly, the statistical uncertainty will scale as $1 / \sqrt{N_{\text {events }}}$. With $53 \mathrm{k}$ 


\section{SLD $R_{b}$ Measurements}

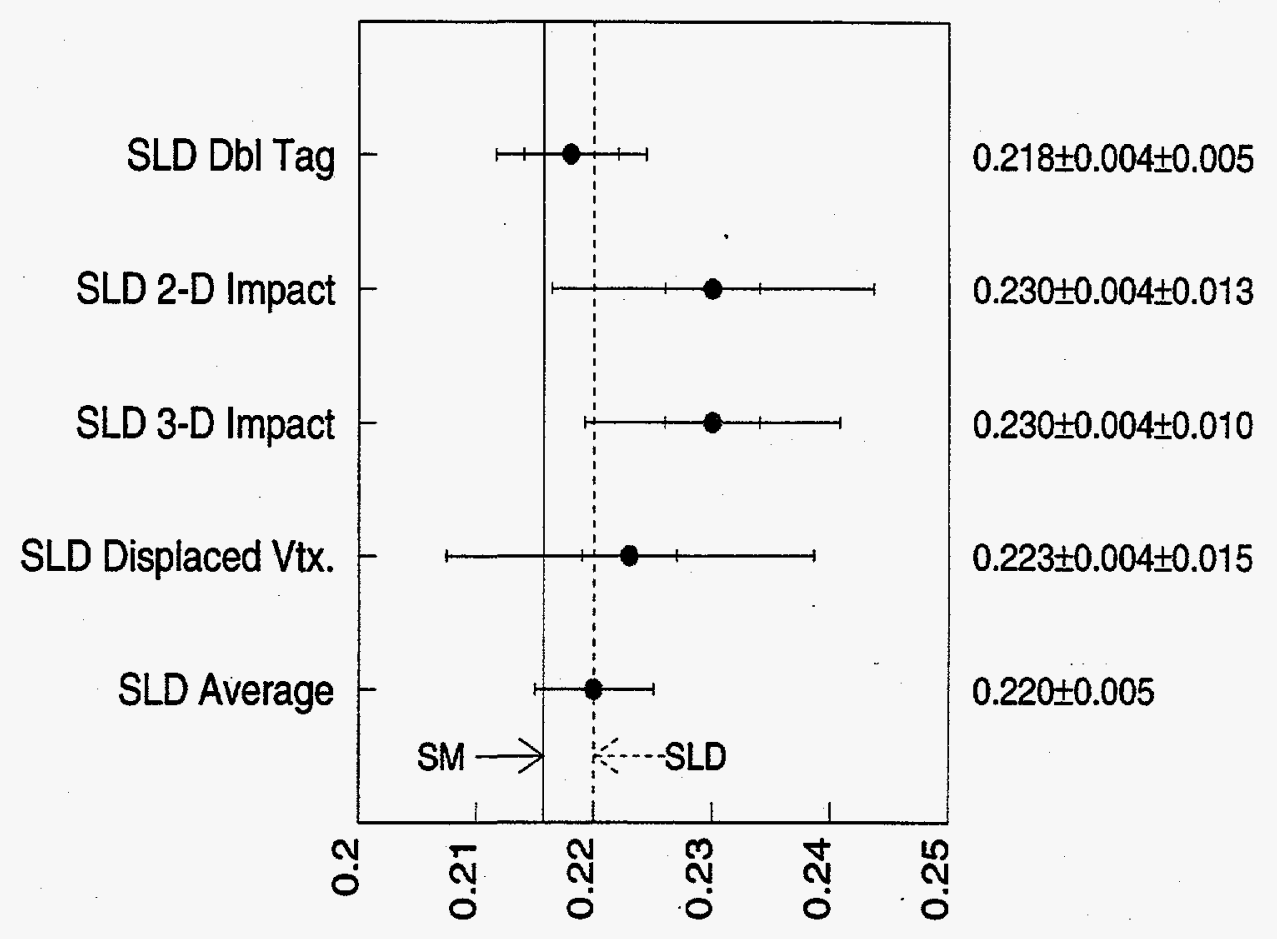

Figure 10.1: SLD $R_{b}$ measurements.

events the combined statistical uncertainty, from all sources, is $6.4 \%$. Thus, if one were to use the current apparatus to make a $\sim 1 \%$ measurement, the number of events needed would be: $53 k \times\left(\frac{6.4 \%}{1.0 \%}\right)^{2}=2.2$ million events. An upgrade to the SLD vertex detector in 1995 should significantly improve the tagging performance and reduce the required number of $Z^{0}$ events. The new version of the vertex detector will have $\sim 50 \%$ better resolution in both the infinite momentum and momentum dependent terms of the $r \phi$ and $r z$ impact parameter resolutions. It is also likely to result in improved track finding efficiencies. In addition, the $\check{C}$ erenkov ring imaging detector of the SLD is now ready for use and may be used to help eliminate backgrounds. The large loss of events to the acceptance cut may be improved in future runs as the new vertex detector will have coverage out to $|\cos \theta|<0.85$ and endcap tracking will 
become possible.

The SLD and LEP $R_{b}$ measurements are shown in Fig. 10.2. The SLD results are consistent with LEP's high $R_{b}$ result. Provided that neither an unidentified bias in the measurements or physical effects such as gluon splitting are not being properly accounted for, the results indicate that new physics may exist. Many models have been proposed which would explain the high $R_{b}$ measurements. With the exception of various SUSY models, most have been eliminated. Many of the SUSY models that can accommodate a high $R_{b}$ value are characterized by the existence of very light SUSY partners $\left(\sim 100 \mathrm{GeV} / c^{2}\right)$. If these do exist it is likely that they will be observed in the near future during the LEP1.5 run starting in 1996.

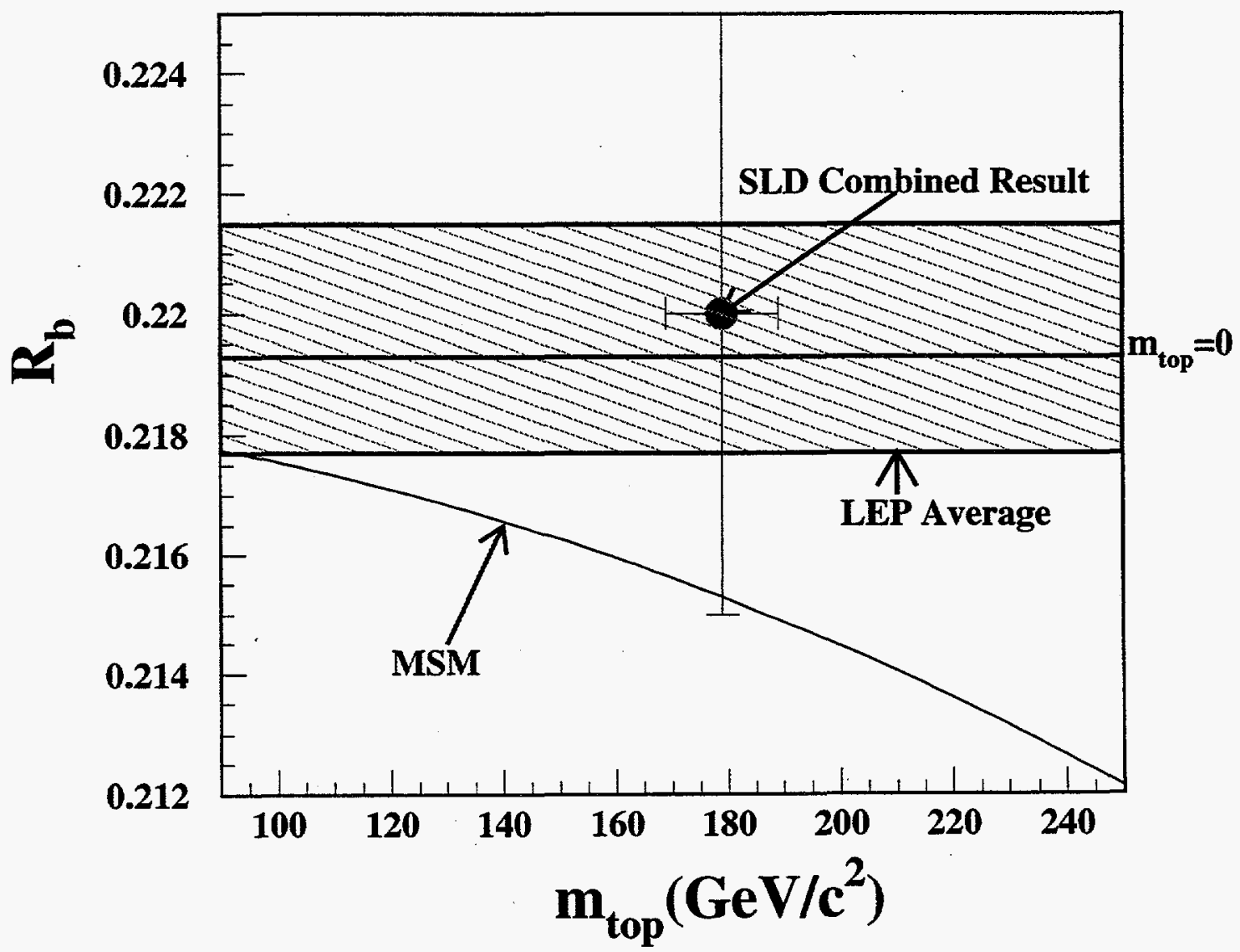

Figure 10.2: Theoretical curves for $R_{b}$ dependence on the top mass with the SLD measurement result added. 


\section{Appendix A}

\section{$V^{0}$ Rejection and Material Simulation Checks}

$V^{0} \mathrm{~s}$ are the 2-prong decays of long lived neutral particles such as $\Lambda$ 's and $K_{s}^{0}$ 's and the conversions of $\gamma$ 's. They account for much of the $b$-tagging of light quarks. Thus, it is necessary to identify these efficiently so that they may be eliminated from the tracks considered in the tags while minimizing misidentification. The $\gamma$ conversions have a side benefit of being useful for verifying the simulation of the detector material. Here, use is made of the fact that $\gamma$ conversions are most likely to occur in the vicinity of a nucleus with which it can impart some momentum. Thus, the rate of $\gamma$ conversions increases as the material density that is being traversed by the $\gamma$ increases. Both the elimination of $V^{0}$ s for purifying the tags and the use of $\gamma$ conversions to test the Monte Carlo material simulation are described below.

\section{A.0.1 Identifying $K_{s}^{0}$ and $\Lambda$ decays}

The procedure for identifying $K_{s}^{0}$ 's and $\Lambda$ 's, uses all pairs of CDC tracks that pass the efficiency cuts and have opposite signed charges. A rough approximation of the $V^{0}$ position in the $x y$ plane is made by treating the tracks as circles in this plane and using the intersecting points. Only CDC tracks are used to avoid biases that may be introduced by a mislinked $V^{0}$ track. However, better resolution than that 


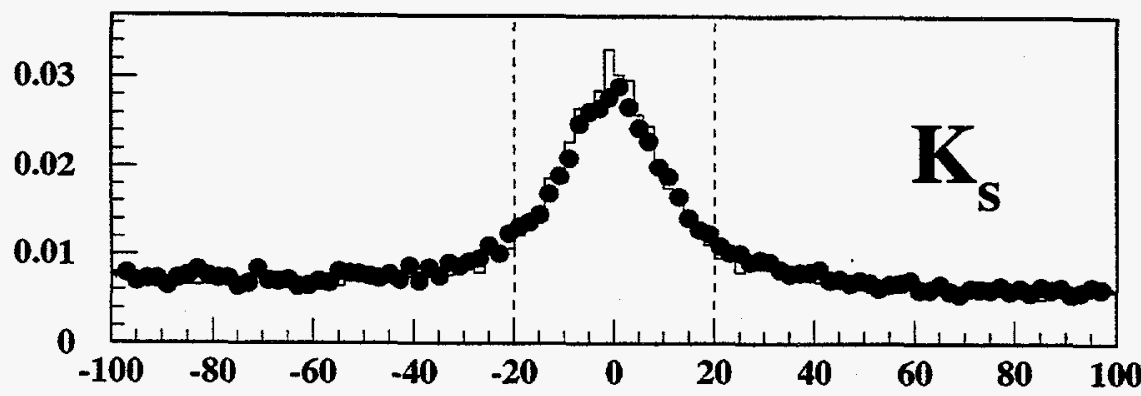

Mass Measured - Mass(K) $\left(\mathrm{MeV} / \mathrm{c}^{2}\right)$
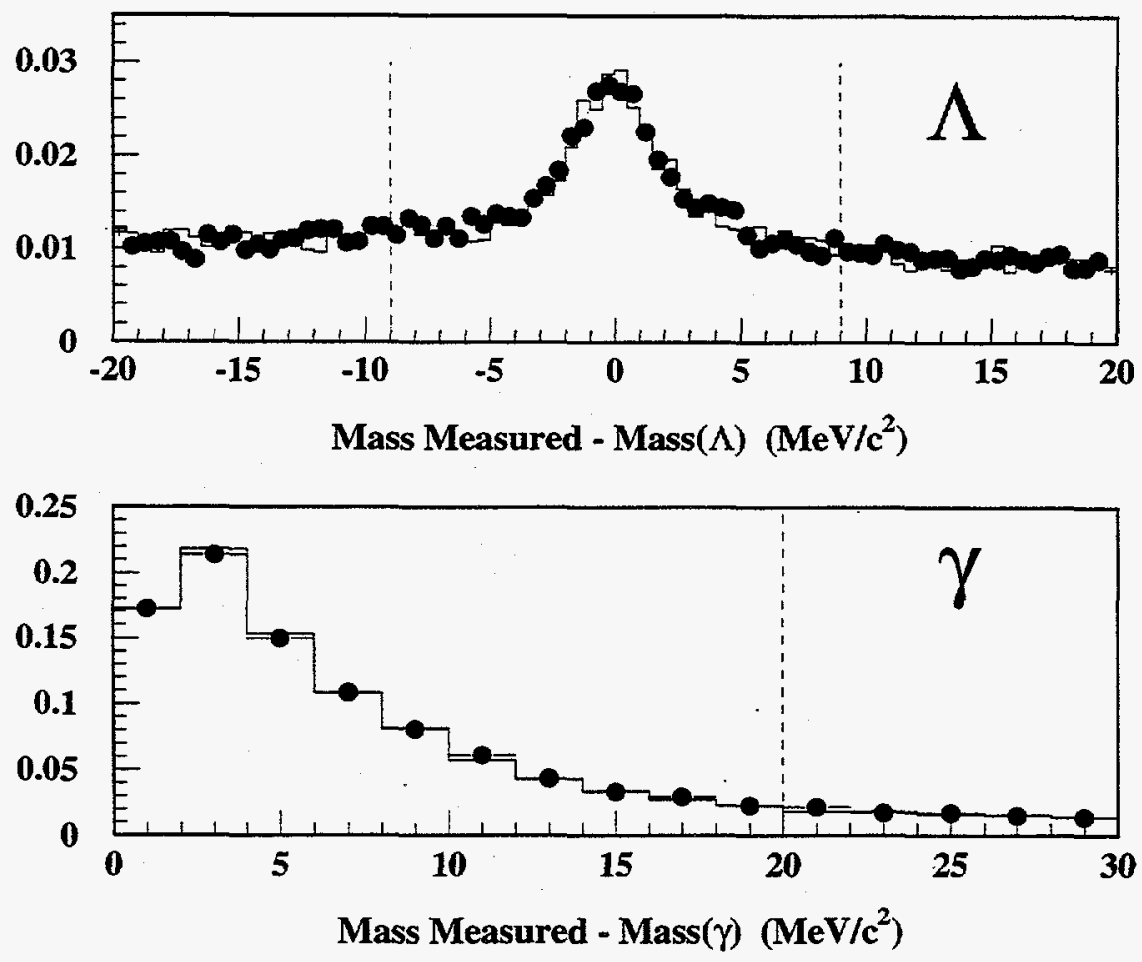

Figure A.1: Mass distributions of identified $V^{0}$ s. The histogram represents the Monte Carlo and the points represent the data.

provided by the $\mathrm{CDC}$ alone is needed below a radius of $2 \mathrm{~cm}$. Therefore, if the crude approximation of the $V^{0}$ position appears to be below this radius, then the $C D C+V X D$ track information is used and the approximate position of the $V^{0}$ vertex is recomputed. Usually two possible vertex positions will be obtained. The most likely vertex is chosen by selecting the vertex with the least longitudinal separation 
of the tracks at the vertex position. Next, the approximate vertex position is used as a seed for performing a complete 3-D vertex fit. Then the fitted parameters are used for the following cuts. The angle between the parent track, taken as going from the primary vertex to the $V^{0}$ position and the momentum vector sum of the $V^{0}$ tracks must be $<0.2(0.5)$ for $K_{s}^{0} \mathrm{~s}(\Lambda \mathrm{s})$. The opening angle of the $V^{0}$ must be less than 1.3 mrad. The separation of the tracks in $x y$ at the vertex position must be less than $0.02 \mathrm{~cm}$. A cut on the longitudinal separation of the tracks is applied with a dependence on the displacement from the primary vertex. Then the invariant mass is determined and the $V^{0}$ passes if the mass is within $0.020 \mathrm{GeV}(0.009 \mathrm{GeV})$ for the $K_{s}^{0}(\Lambda)$. Finally, since $K_{s}^{0} \mathrm{~s}$ are more copious than $\Lambda \mathrm{s}$, a $\Lambda$ candidate is eliminated if one of its tracks also belongs to a $K_{s}^{0}$ candidate. An improvement of about $7 \%$ in the $b$-purity $\left(\pi_{b}\right)$ of the 2-D Impact Parameter Event Tag $b$-tagged sample is observed with about a $1 \%$ loss in the $b$-tagging efficiency.

\section{A.0.2 Identifying Gamma Conversions}

The procedure for identifying $\gamma$ conversions is similar to that for $K_{s}^{0}$ 's and $\Lambda$ 's except that the position of the conversion is determined by where the circle approximations of the corresponding tracks in the $x y$ plane are parallel to each other. This was determined to be better than using the crossing point because of the very small opening angle resulting from the massless parent particle(the $\gamma$ ). Tracking resolution can cause the tracks to appear not to intersect and the uncertainty in position along the $\gamma \mathrm{s}$ flight direction can be very large (several $\mathrm{cm}$ ). A full vertex fit is not performed. Similarly to the procedure for $K_{s}^{0}$ 's and $\Lambda$ 's, the following cuts are performed. The angle between the parent track, taken as going from the primary vertex to the $V^{0}$ position, and the momentum vector sum of the $V^{0}$ tracks must be $<0.2$. The opening angle of the $V^{0}$ must be less than 0.2 mrad. The separation of the tracks in $x y$ at the vertex position must be less than $0.3 \mathrm{~cm}$. Then the invariant mass is determined and the $\gamma$ is identified if this mass is less than $0.020 \mathrm{GeV}$. 


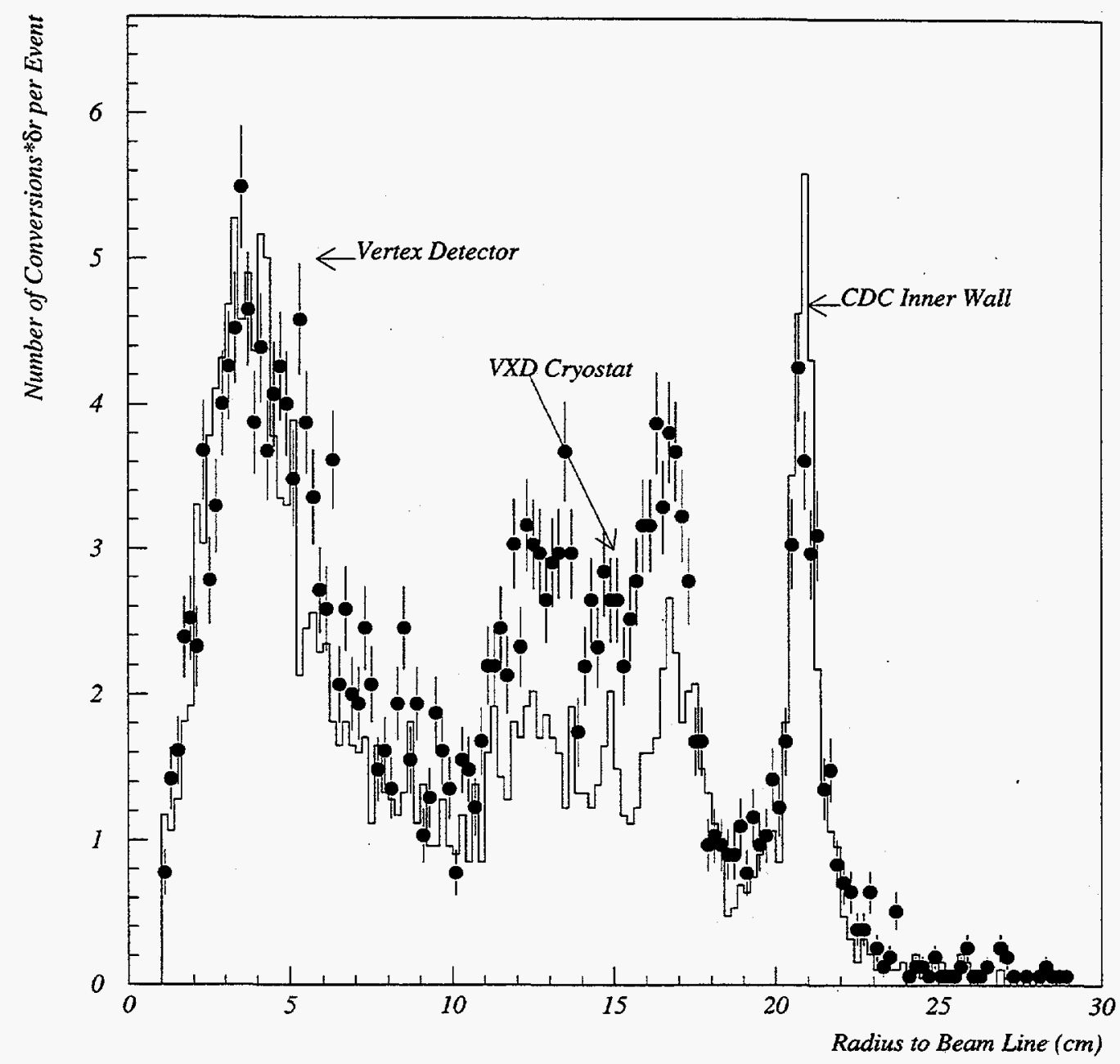

Figure A.2: Gamma conversions rates distance from beam-line for the data(points) and Monte Carlo(histogram).

\section{A.0.3 Using Conversions to test Monte Carlo Material Sim- ulation}

The density of identified $\gamma$ conversions per $Z^{0}$ event was histogrammed versus the coordinates of the detector for data and Monte Carlo to check for differences in material density. The method is restricted to regions of the detector where $\gamma$ conversions 
DATA

$\ddot{8}$

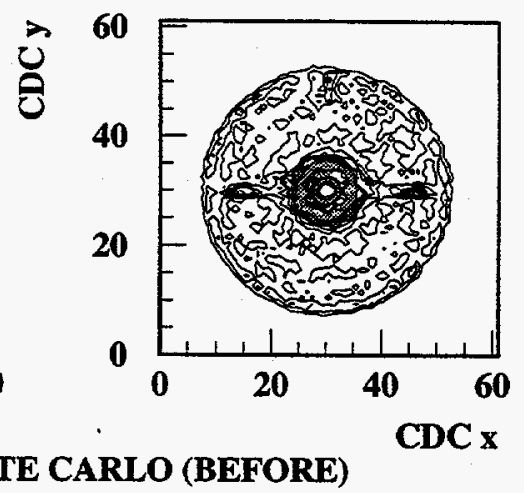

荌

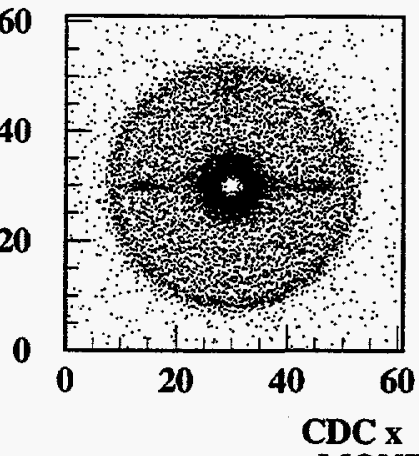

MONTE CARLO (BEFORE)
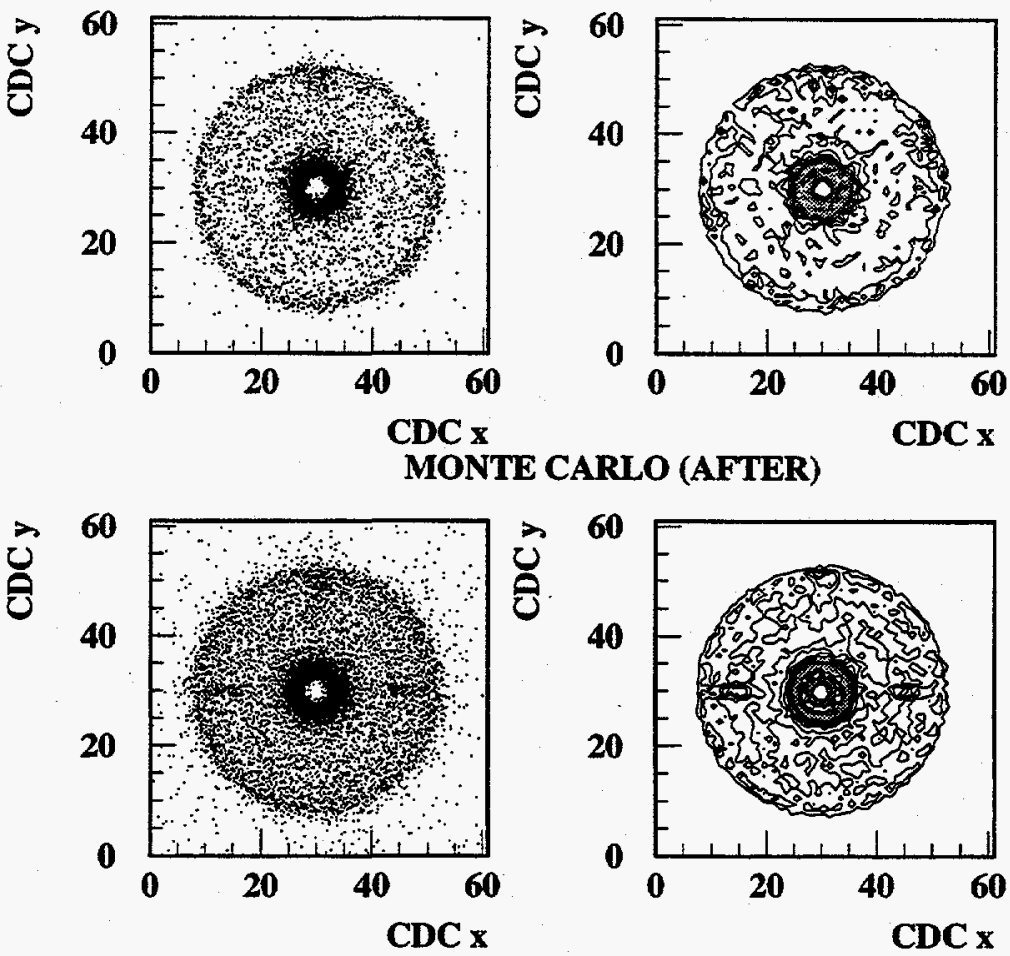

Figure A.3: Scatter plot of density of gamma conversion found in the region of the beam pipe to the inner wall of the CDC.

could be identified (i.e. the central region before the CRID). When this method was first applied to the Monte Carlo several problems were quickly identified. Unlike Fig. A.2 of the $\gamma$ conversion density versus radius which shows the corrected Monte Carlo, a very noticeable discrepancy in the position of the CDC's inner wall existed. Fig. A. 3 is a scatter plot of the position of the gamma conversions in the $x y$ plane. The 
first row shows the data. The second row shows the Monte Carlo after the inner wall simulation was fixed but another problem was identified. A large difference exists in the population between the CDC and VXD where the supporting styrofoam for the cryostat. The styrofoam discrepancy was traced to a misunderstanding of the styrofoam composition. Other problems involving improperly simulated bolts and seams were also identified by this method and corrected. Other projections of the gamma conversion distributions are shown in Fig. A.4. The dark streaks correspond to steel bands around the VXD cryostat and the bolt for holding the halves of the cryostat together. 

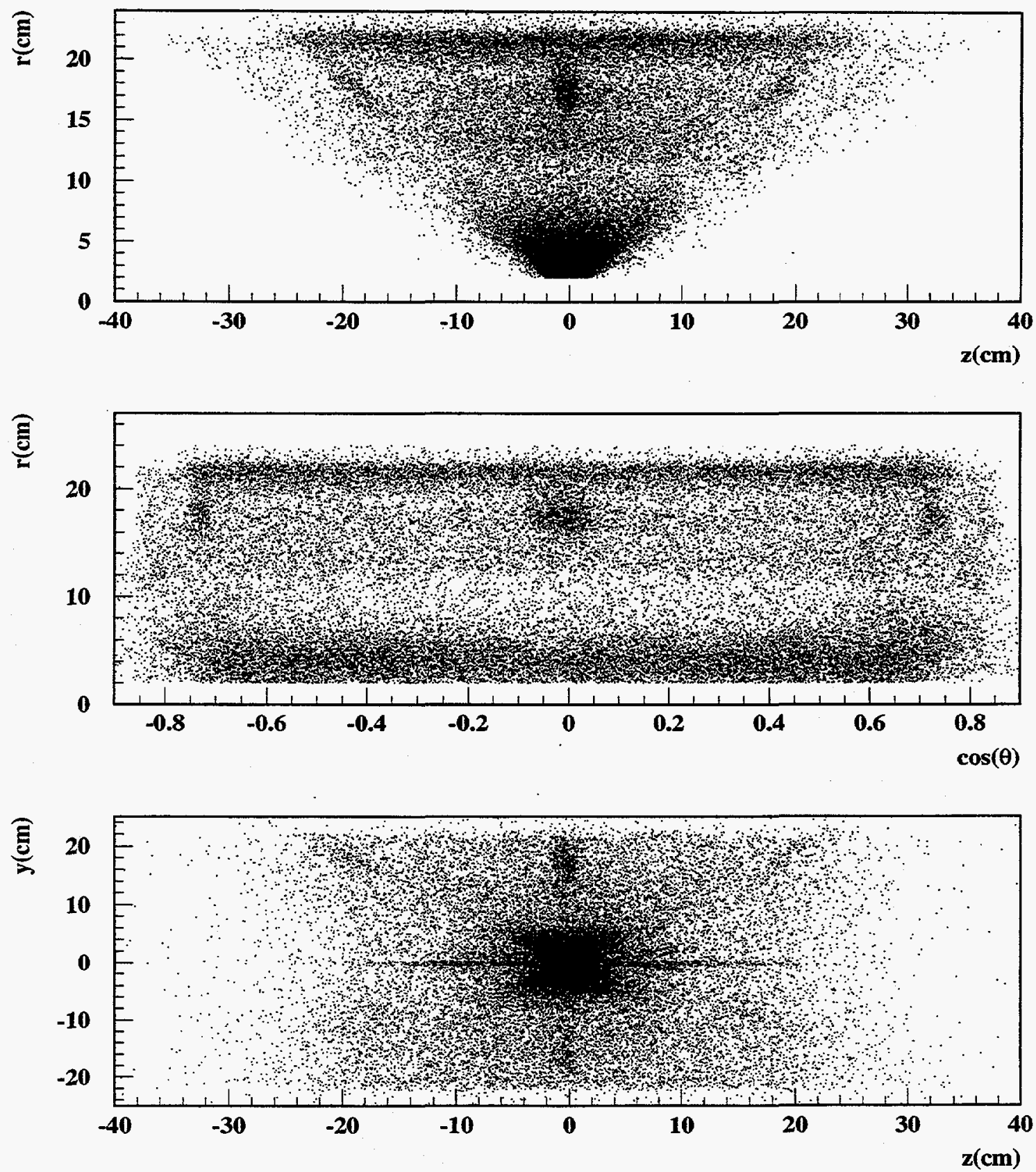

Figure A.4: Projections of the gamma conversion position distribution. 


\section{Appendix B}

\section{Other Procedures for Measuring $R_{b}$ at SLD}

Other tagging procedures were studied for measuring $R_{b}$ with the SLD. Two of them are event tags similar to the 2-D Impact Parameter Event Tag. These are the 3-D Impact Parameter Event Tag[56] and the 3-D displaced vertex tag[57]. These were found to yield results competitive with the 2-D Impact Parameter Event Tag. Another method studied was the impact parameter sum method devised by MARKII[60]. These are briefly described below.

\section{B.0.4 3-D Impact Parameter Event Tag}

The 3-D Impact Parameter Event Tag was performed in a similar fashion to the 2$\mathrm{D}$ impact parameter analysis but with the full 3-D information used for the impact parameter and for signing [56]. As shown in Fig. B.1 the tag was significantly more pure for the same efficiency. Thus, while being able to maintain a statistical uncertainty comparable to that from the 2-D Impact Parameter Event Tag the systematics from the udsc contamination was reduced. Also, it turned out to be less sensitive to resolution effects. This was interpreted as the amount of resolution degradation being less significant compared to the longer length being measured. The result had a systematic uncertainty that was $30 \%$ less but like the 2-D Impact Parameter Event 
2-D Impact Parameter Tag
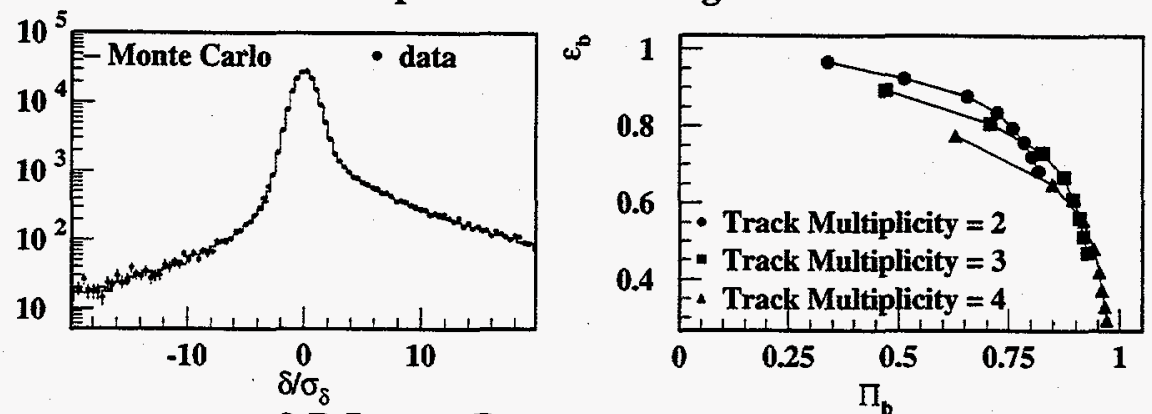

3-D Impact Parameter Tag
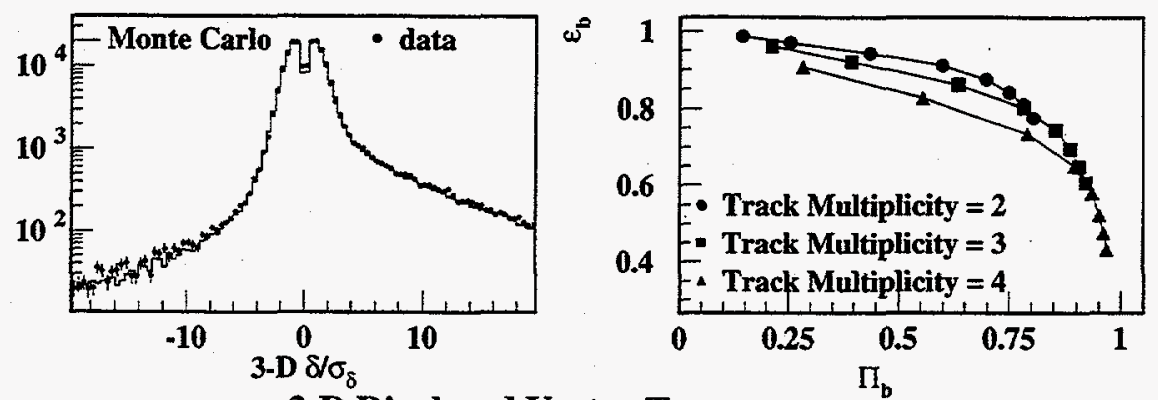

3-D Displaced Vertex Tag
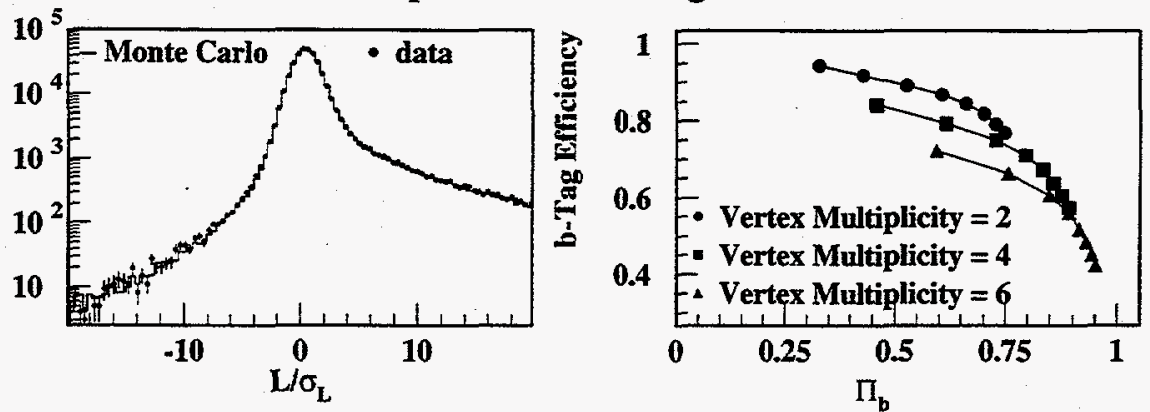

Figure B.1: Distributions of tagging variable and the resulting $b$-tagging performance for event tags used at SLD.

Tag it is systematics limited due to the dependence on the $B$-decay model. The published result from this technique is shown in Fig. 10.1. 


\section{B.0.5 3-D Displaced Vertex Tag}

The 3-D Displaced Vertex Tag was developed and implemented by Su Dong. It was one of the first analyses to take advantage of the excellent vertexing capabilities provided by the high precision of the SLD tracking systems[57]. In this method the tag is a cut on the number of vertices, made from pairs of tracks within a jet, that are significantly displaced from the primary vertex. The significance of the displacement of the vertices from the primary vertex is show in Fig. B.1. The sign is obtained from the dot product of the momentum sum of the vertex with the vector from the primary vertex to the vertex. The performance of the tag was comparable to the 2-D Impact Parameter Event Tag and was less sensitive to uncertainties in the primary vertex position. However, it did suffer from a high sensitivity to tracking efficiencies and decay multiplicities. This was due to the fact that with the vertices there was a chance of missing them if either track was lost due to an inefficiency. The published result from this technique is shown in Fig. 10.1.

\begin{tabular}{|l|c|}
\hline Result & Value \pm Statistical Uncertainty \\
\hline$R_{b}$ & $0.2345 \pm 0.0094$ \\
$R_{c}$ & $0.1590 \pm 0.0200$ \\
$R_{u d s}$ & $0.6066 \pm 0.0165$ \\
$\chi^{2} /$ n.d.f. & $81 / 69$ \\
\hline
\end{tabular}

\section{B.0.6 Impact Parameter Sum}

In the impact parameter sum method[60] the mean significance of the data events are histogrammed and the resulting distributions are fitted via a Maximum Likelihood technique to the $u d s$ and $c$ Monte Carlo distributions and a parameterized fit to the $b$ distribution. The exponential in the parameterization of the $b$ distribution is allowed to float so as to reduce sensitivity to the $b$-lifetime. In Fig. B.2 the result of the maximum likelihood fit is shown overlayed on the data with the distributions for $u d s$ and $u d s+c$ also overlayed. The method has the advantage of measuring $R_{b}, R_{c}$ and 
Fit to Impact Sum

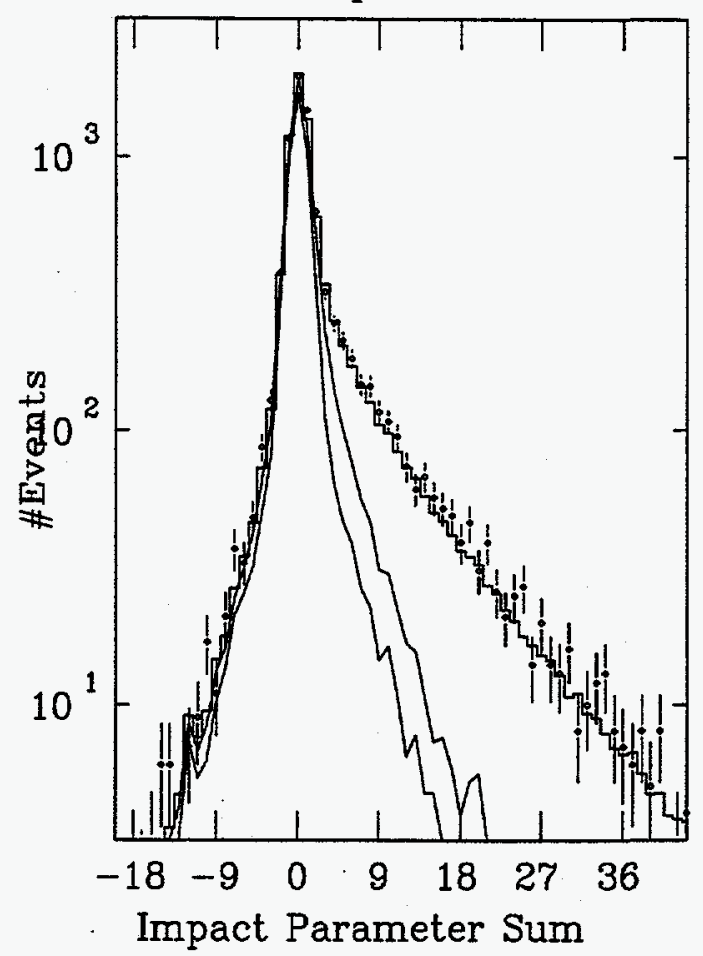

Components of Fit

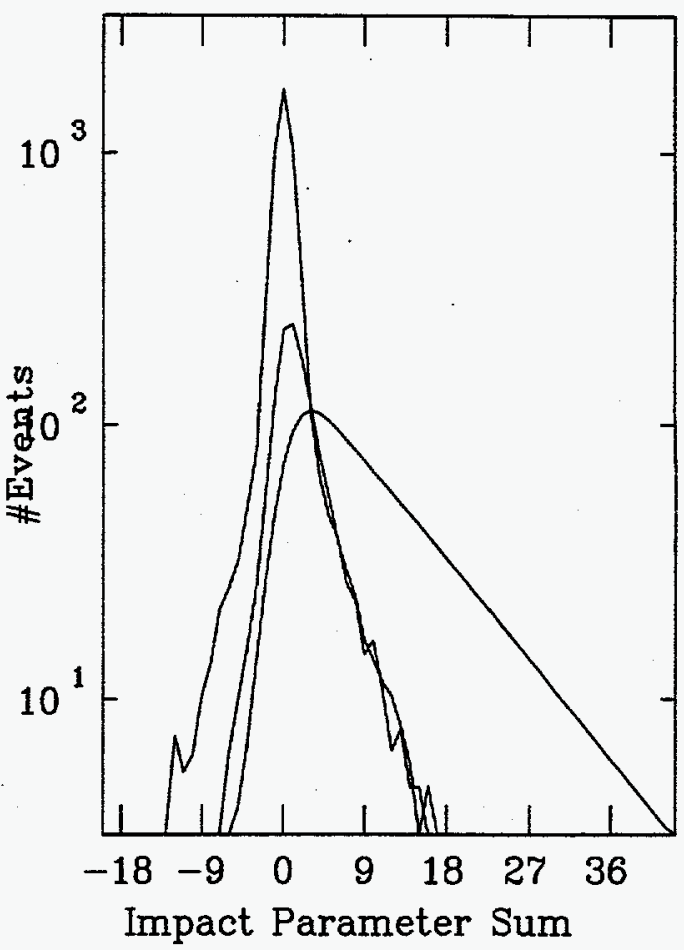

16:22:06 1014 r95

Figure B.2: Mlustration of the impact parameter sum method where the $b, c$ and $u d s$ distributions have been fit to the data to measure the branching fractions.

$R_{u d s}$ simultaneously. Unfortunately, it was determined that the result was very model dependent. The results without systematical uncertainties are shown in Table B.0.5. 


\section{Appendix $\mathrm{C}$}

\section{Drift Chamber Monitoring and Calibrations}

The performance of the chamber is sensitive its pressure, temperature and gas mixture. Through a precision system the temperature and gas mixture are controlled and monitored. The variation of the chamber pressure with the atmospheric pressure and occasional fluctuations in the gas mixture significantly affect the track reconstruction quality. To compensate, frequently updated calibration constants are used to maintain a better performance. The pressure, temperature, and gas mixture most directly affect the relationship of the times of the drift chamber hits to the actual distance from the cell plane that they correspond to. The major effect of this is controlled by the drift velocity correction which behaves as a scale in the time to distance relationship.

\section{C.0.7 Drift Velocity Constants}

The time to distance relationship is determined by what is termed the drift model. For the nominal conditions of the detector it will estimate the drift distance associated with the measured drift time for a CDC hit. However, the relation varies with the $\mathrm{CDC}$ environment. To correct for this a scale which reflects changes in gas content, pressure, or temperature is used. The dimensionless drift velocity correction becomes 


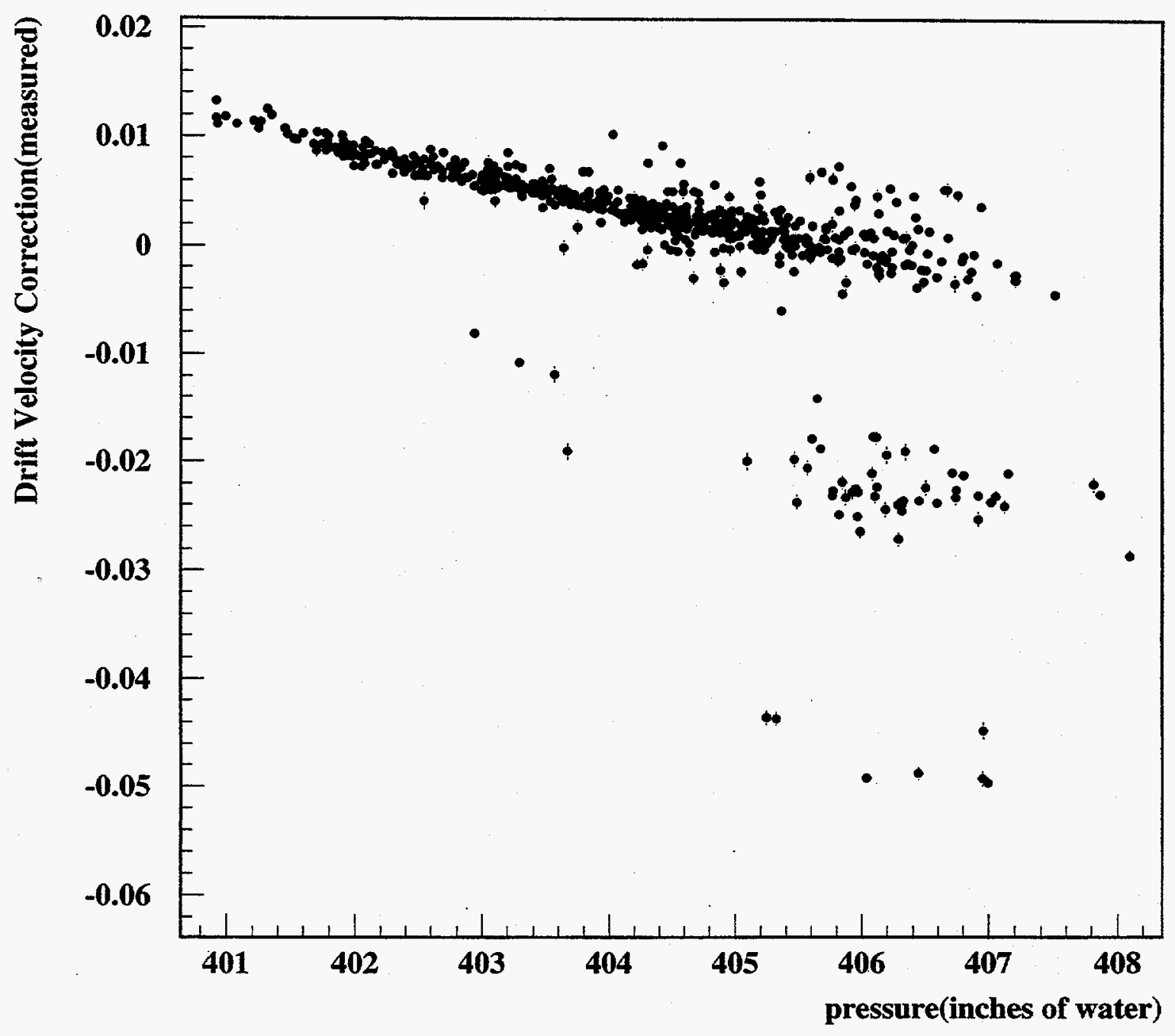

Figure C.1: Demonstration of the strong linear dependence of the drift velocity on the pressure of the gas.

a multiplicative scale to the measured drift times and distances as follows:

$$
t_{\text {scale }}=1 /\left(1+\delta V_{d}\right)
$$


true drift time $=t^{\text {measured }} \times t_{\text {scale }}$

$$
\text { true drift distance }=d^{\text {measured }} \times t_{\text {scale }}
$$

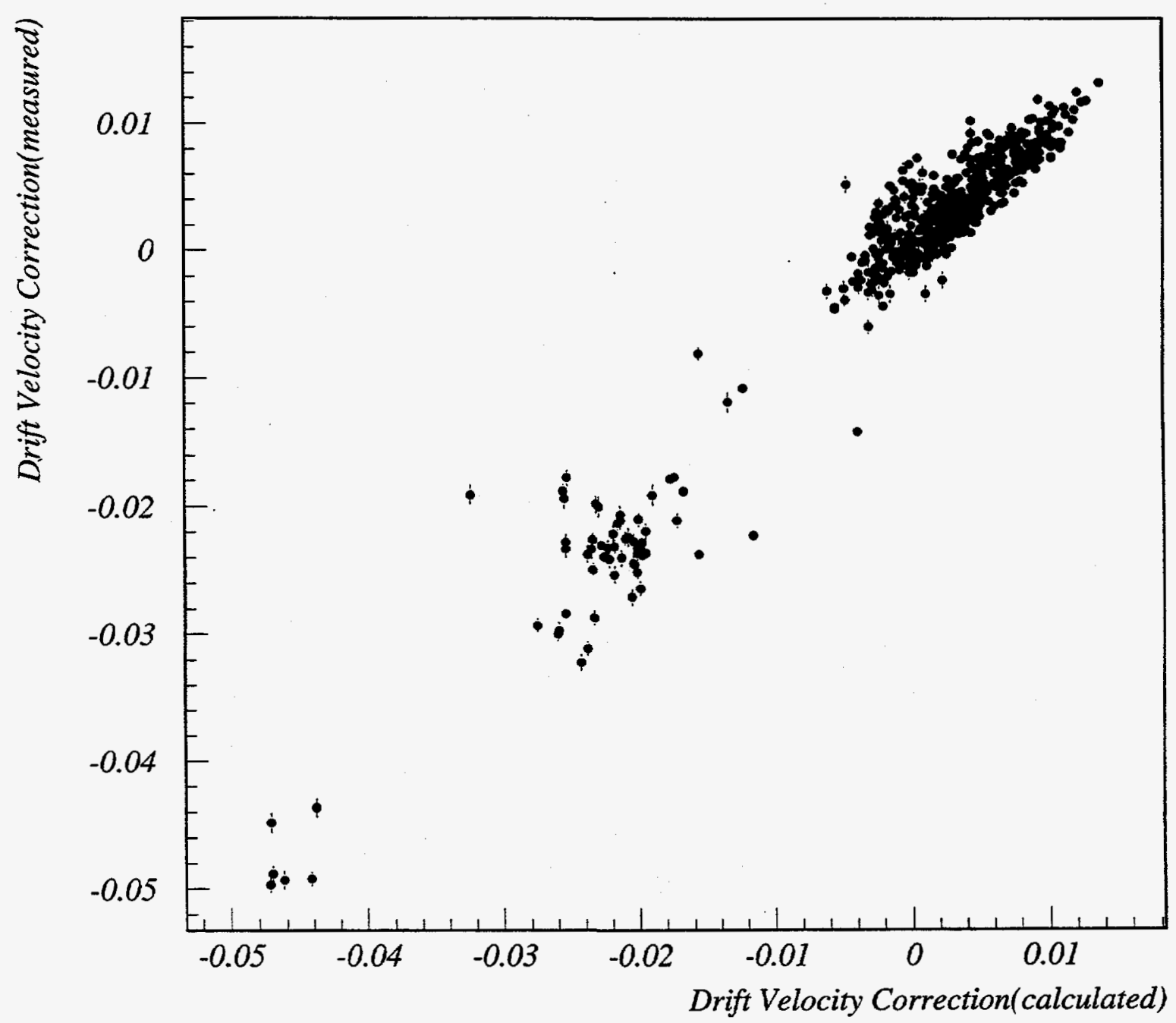

Figure C.2: The comparison of the measured drift velocity to the calculated drift velocity shows that a simple linear equation with inputs of the monitored chamber pressure, water content of the gas, and temperature can accurately determine the correction. 


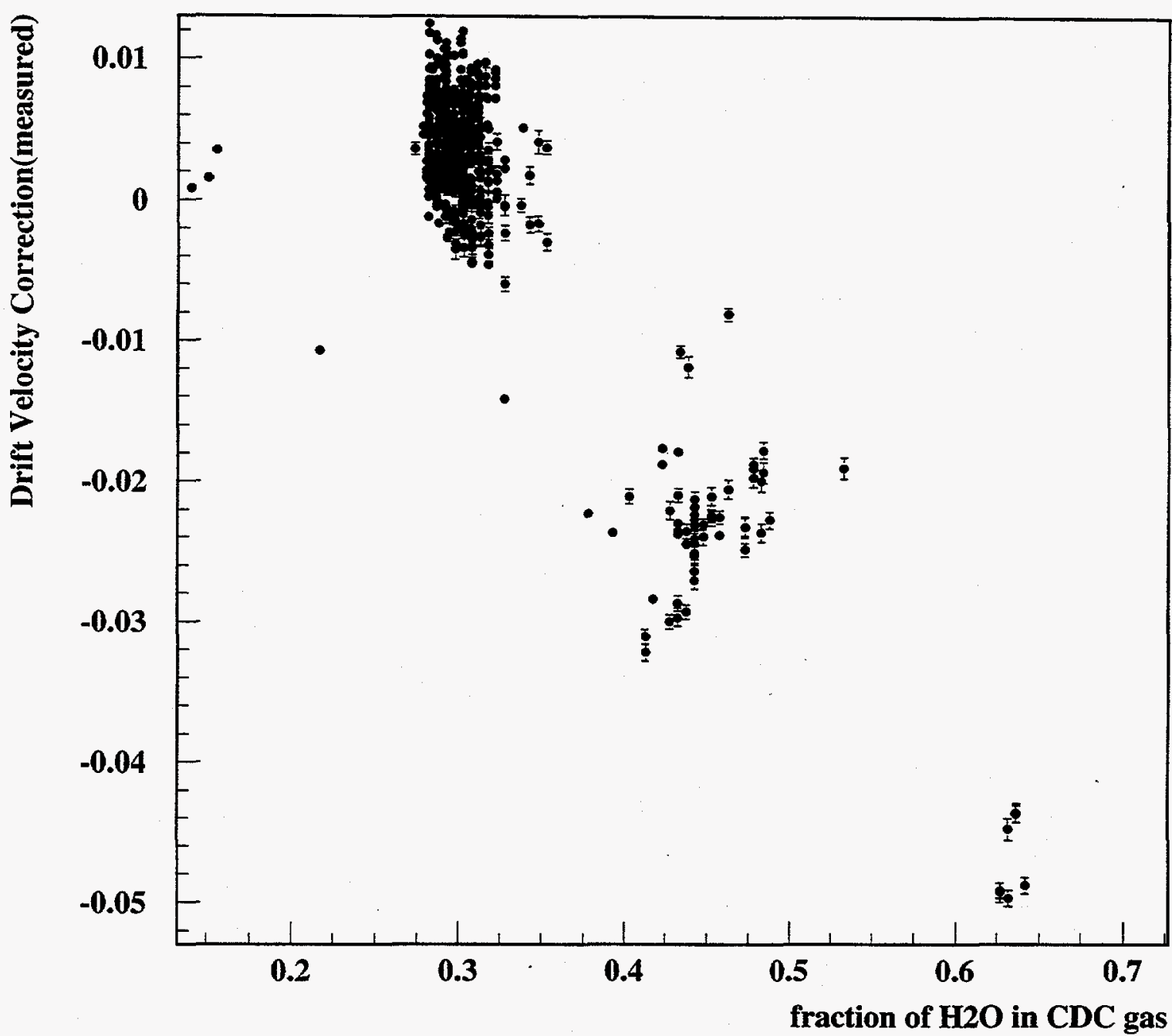

Figure C.3: Dependence of drift velocity correction on water content of the CDC gas.

The drift velocity varies, on average, by $\sim 0.5 \%$ during a day. The nominal drift velocity is $7.9 \mu \mathrm{m}$ per nanosecond. The drift velocity correction is normally obtained by having it as a floating parameter in the track fits. From this a drift velocity for each track is obtained. However, the error on any of these measurements is large $(\sim 0.013)$. A more accurate drift velocity is obtained by performing a weighted average of the drift velocities of all tracks in a run that appear to originate from the primary vertex 


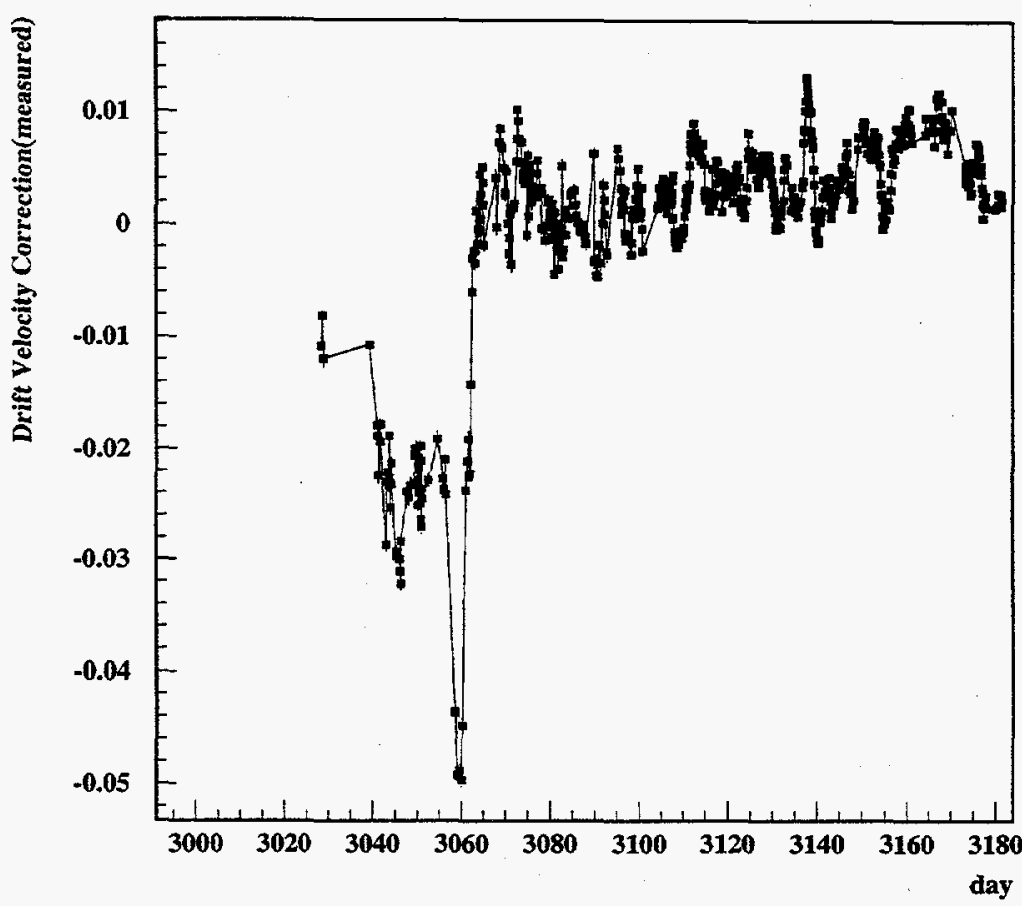

Figure C.4: The drift velocity correction versus time. The dip corresponds to a time when the water content was maladjusted and indicates the strong dependence of the drift velocity on this.

and have a $p_{x y}>0.5 \mathrm{GeV} / c$. By doing this an uncertainty on the drift velocity for a run of $\sim 0.25 \%$ is typically obtained.

As a result of studies to understand a large fluctuation in the drift velocities during the beginning of the 1993 SLD run it was discovered that the drift velocity is mostly dependent on the pressure and water content of the gas. A flow control problem had resulted in the water content of the gas increasing from $0.312 \%$ to $0.632 \%$ causing a change of -0.0518 to to drift velocity correction. The relation was easily identified by scatter plotting monitored gas quantities versus the drift velocity. From these the strong correlation of to the pressure and hygrometer( $\mathrm{H} 2 \mathrm{O}$ content measurement) were identified as shown in Fig. C.3. Subsequently, an attempt at predicting the drift velocity from these monitored quantities was made by assuming a linear relation of the drift velocity to the monitored values and performing a least squares fit. A 
dependence on the temperature of the gas was also expected and thus was included in the fit. The result was a simple procedure for predicting the drift velocity to an accuracy approaching that of fitting for the drift velocity. The calculated versus measured drift velocity results are shown in Fig. C.2. The drift velocity correction is given by:

$$
\begin{array}{r}
\delta V_{d}=-0.0026850\left(\text { inches of } H_{2} O\right)^{-1} \times \text { pressure } \\
-0.14472 \times \text { water content }+0.0017830 C^{\circ-1}+1.0944
\end{array}
$$

\section{C.0.8 Charge Asymmetry Calibrations}

To account for differences in responses of mother boards between charge readout on the north end and south end of the sense wires and to identify cases where the readout on either or both ends may be faulty a calibration of the longitudinal position of the hit versus the measured charge asymmetry is performed. A simple linear model with an effective offset and wire length for each wire is used. The procedure uses hits on fitted tracks which have valid north and south charge deposition information. To avoid contamination from other nearby hits and any spurious hits formed from the trailing edge of a waveform, the hits are required to come from wires with only one hit on a track and only first hits on wires.[58] 


\section{Appendix D}

\section{Effect of Backgrounds on Tracking}

The various track quantities are checked for dependence on the level of the beam related background. The background level is taken from random events near the data events passing the event selection and from the random events used for the Monte Carlo event generation. For the data one is assuming that the background level is typical of a long period since the random event may be up to a minute from the occurence of the $Z^{0}$ in the data. The quantities from the random events that were used as estimators of the background level were the energy in the calorimeter, the number of cells hit in the CDC and the number of pixels with hits in the VXD. Using the random events prevents contamination from any actual physics process that would cause fluctuations in these counts. As a function of these counts, the number of good CDC tracks, number of good CDC tracks that linked per event, and the $b$-tagging rate were studied. The only significant variation observed is in the linking rate versus the noise counts as shown in Fig. D.1. However, this appears to be well modelled in the Monte Carlo. 


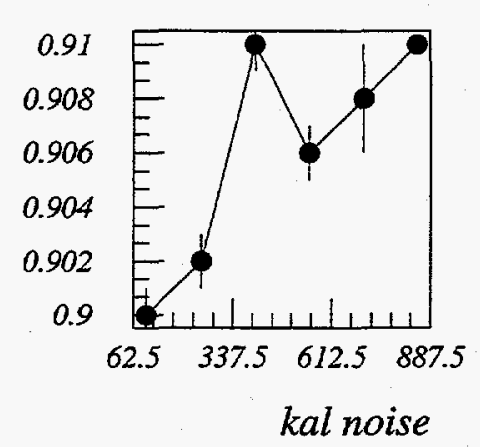

link rate for good mc tracks
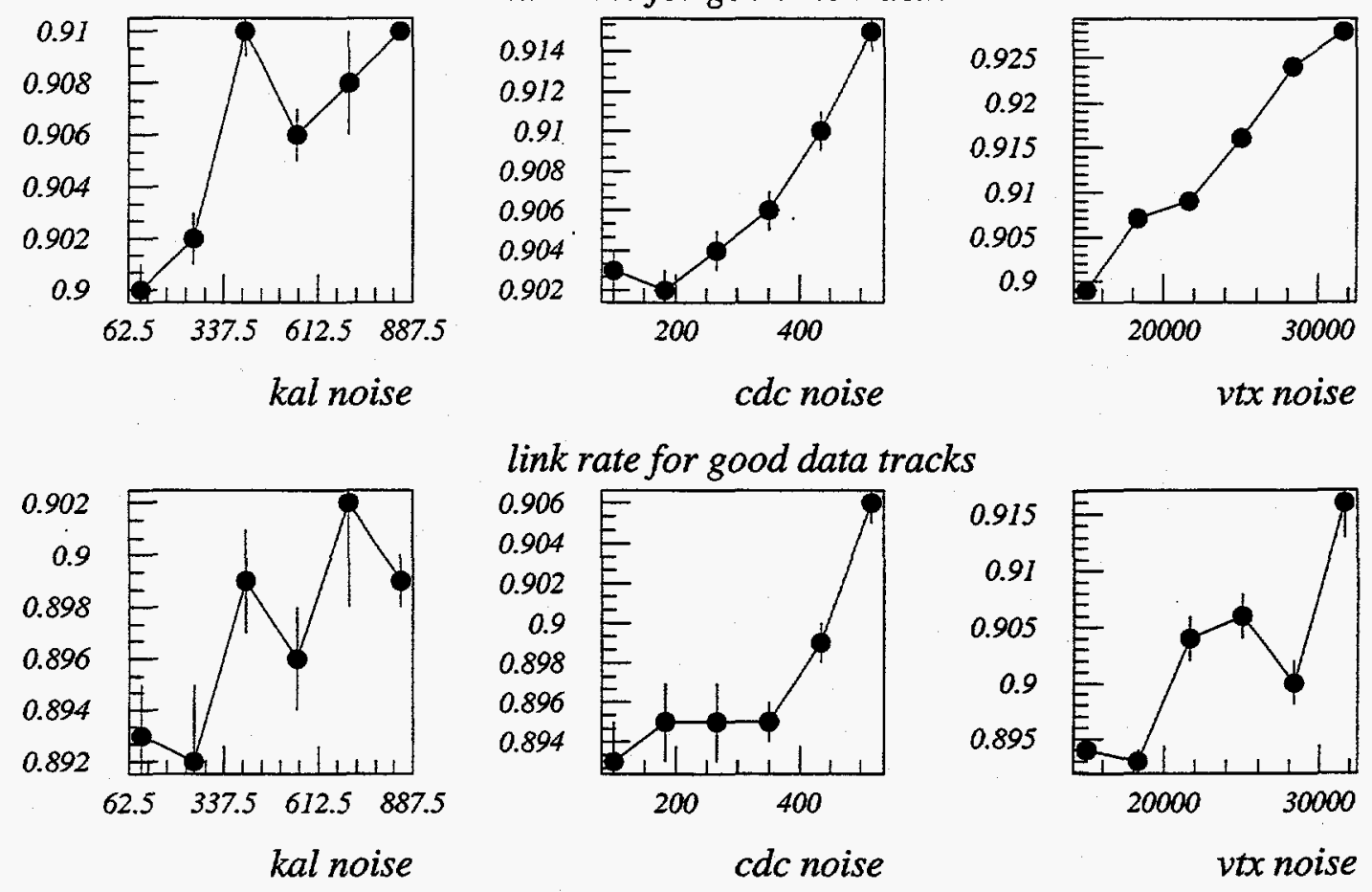

link rate for good data tracks
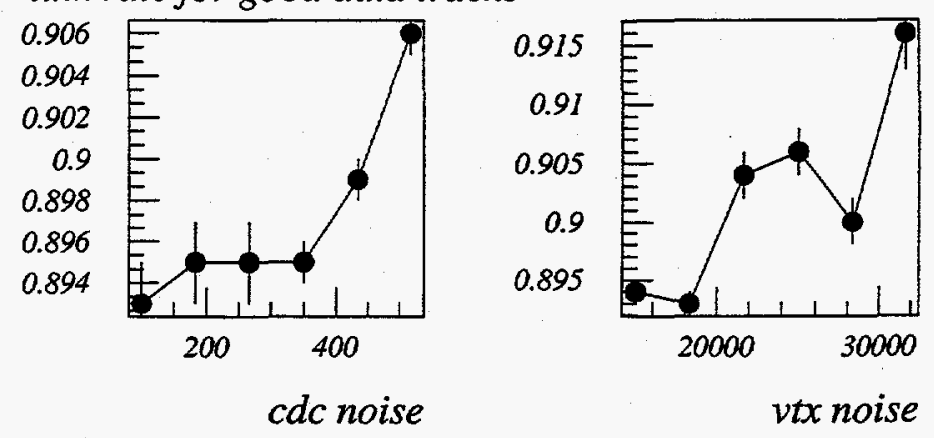

Figure D.1: Variation of linking rate versus noise counts in the calorimeter, CDC and VXD for the Monte Carlo and data. 


\section{Appendix E}

\section{The SLD Collaboration}

${ }^{*}$ K. Abe, ${ }^{(29)}$ I. Abt, ${ }^{(14)}$ C.J. Ahn, ${ }^{(26)}$ T. Akagi, ${ }^{(27)}$ N.J. Allen, ${ }^{(4)}$ W.W. Ash,,${ }^{(27) \dagger}$ D. Aston, ${ }^{(27)}$ K.G. Baird,,${ }^{(25)}$ C. Baltay, ${ }^{(33)}$ H.R. Band, ${ }^{(32)}$ M.B. Barakat, ${ }^{(33)}$ G. Baranko, ${ }^{(10)}$ O. Bardon, ${ }^{(16)}$ T. Barklow, ${ }^{(27)}$ A.O. Bazarko, ${ }^{(11)}$ R. Ben-David, ${ }^{(33)}$ A.C. Benvenuti, ${ }^{(2)}$ T. Bienz, ${ }^{(27)}$ G.M. Bilei, ${ }^{(22)}$ D. Bisello, ${ }^{(21)}$ G. Blaylock, ${ }^{(7)}$ J.R. Bogart, ${ }^{(27)}$ T. Bolton, ${ }^{(11)}$ G.R. Bower, ${ }^{(27)}$ J.E. Brau, ${ }^{(20)}$ M. Breidenbach, ${ }^{(27)}$ W.M. Bugg, ${ }^{(28)}$ D. Burke, ${ }^{(27)}$ T.H. Burnett, ${ }^{(31)}$ P.N. Burrows, ${ }^{(16)}$ W. Busza, ${ }^{(16)}$ A. Calcaterra, ${ }^{(13)}$ D.O. Caldwell, ${ }^{(6)}$ D. Calloway, ${ }^{(27)}$ B. Camanzi, ${ }^{(12)}$ M. Carpinelli, ${ }^{(23)}$ R. Cassell, ${ }^{(27)}$ R. Castaldi, ${ }^{(23)(a)}$ A. Castro, ${ }^{(21)}$ M. Cavalli-Sforza, ${ }^{(7)}$ E. Church, ${ }^{(31)}$ H.O. Cohn, ${ }^{(28)}$ J.A. Coller, ${ }^{(3)}$ V. Cook, ${ }^{(31)}$ R. Cotton, ${ }^{(4)}$ R.F. Cowan, ${ }^{(16)}$ D.G. Coyne, ${ }^{(7)}$ A. D'Oliveira, ${ }^{(8)}$ C.J.S. Damerell, ${ }^{(24)}$ M. Daoudi, ${ }^{(27)}$

R. De Sangro, ${ }^{(13)}$ P. De Simone, ${ }^{(13)}$ R. Dell'Orso, ${ }^{(23)}$ M. Dima, ${ }^{(9)}$ P.Y.C. Du, ${ }^{(28)}$ R. Dubois, ${ }^{(27)}$ B.I. Eisenstein, ${ }^{(14)}$ R. Elia, ${ }^{(27)}$ E. Etzion, ${ }^{(4)}$ D. Falciai, ${ }^{(22)}$ M.J. Fero, ${ }^{(16)}$ R. Frey, ${ }^{(20)}$ K. Furuno, ${ }^{(20)}$ T. Gillman, ${ }^{(24)}$ G. Gladding, ${ }^{(14)}$ S. Gonzalez, ${ }^{(16)}$ G.D. Hallewell, ${ }^{(27)}$ E.L. Hart, ${ }^{(28)}$ Y. Hasegawa, ${ }^{(29)}$ S. Hedges, ${ }^{(4)}$ S.S. Hertzbach, ${ }^{(17)}$ M.D. Hildreth, ${ }^{(27)}$ J. Huber, ${ }^{(20)}$ M.E. Huffer,${ }^{(27)}$ E.W. Hughes, ${ }^{(27)}$ H. Hwang, ${ }^{(20)}$ Y. Iwasaki, ${ }^{(29)}$ D.J. Jackson, ${ }^{(24)}$ P. Jacques, ${ }^{(25)}$ J. Jaros, ${ }^{(27)}$ A.S. Johnson, ${ }^{(3)}$ J.R. Johnson, ${ }^{(32)}$ R.A. Johnson, ${ }^{(8)}$ T. Junk, ${ }^{(27)}$ R. Kajikawa, ${ }^{(19)}$ M. Kalelkar, ${ }^{(25)}$ H. J. Kang, ${ }^{(26)}$ I. Karliner, ${ }^{(14)}$ H. Kawahara, ${ }^{(27)}$ H.W. Kendall, ${ }^{(16)}$ Y. Kim, ${ }^{(26)}$ M.E. King, ${ }^{(27)}$ R. King, ${ }^{(27)}$ R.R. Kofler, ${ }^{(17)}$ N.M. Krishna, ${ }^{(10)}$ R.S. Kroeger, ${ }^{(18)}$ J.F. Labs, ${ }^{(27)}$ M. Langston, ${ }^{(20)}$ A. Lath,,${ }^{(16)}$ J.A. Lauber, ${ }^{(10)}$ 
D.W.G. Leith, ${ }^{(27)}$ M.X. Liu, ${ }^{(33)}$ X. Liu, ${ }^{(7)}$ M. Loreti, ${ }^{(21)}$ A. Lu, ${ }^{(6)}$ H.L. Lynch, ${ }^{(27)}$ J. Ma, ${ }^{(31)}$ G. Mancinelli, ${ }^{(22)}$ S. Manly, ${ }^{\left({ }^{(3)}\right.}$ G. Mantovani, ${ }^{(22)}$ T.W. Markiewicz, ${ }^{(27)}$ T. Maruyama, ${ }^{(27)}$ R. Massetti, ${ }^{(22)}$ H. Masuda, ${ }^{(27)}$ E. Mazzucato, ${ }^{(12)}$ A.K. McKemey, ${ }^{(4)}$ B.T. Meadows, ${ }^{(8)}$ R. Messner, ${ }^{(27)}$ P.M. Mockett, ${ }^{(31)}$ K.C. Moffeit, ${ }^{(27)}$ B. Mours, ${ }^{(27)}$ G. Müller, ${ }^{(27)}$ D. Muller, ${ }^{(27)}$ T. Nagamine, ${ }^{(27)}$ U. Nauenberg, ${ }^{(10)}$ H. Neal, ${ }^{(27)}$ M. Nussbaum, ${ }^{\left({ }^{(8)}\right.}$ Y. Ohnishi, ${ }^{(19)}$ L.S. Osborne, ${ }^{(16)}$ R.S. Panvini, ${ }^{(30)}$ H. Park, ${ }^{(20)}$ T.J. Pavel, ${ }^{(27)}$ I. Peruzzi, ${ }^{(13)(6)}$ M. Piccolo, ${ }^{(13)}$ L. Piemontese, ${ }^{(12)}$ E. Pieroni, ${ }^{(23)}$ K.T. Pitts, ${ }^{(20)}$ R.J. Plano, ${ }^{(25)}$ R. Prepost, ${ }^{(32)}$ C.Y. Prescott, ${ }^{(27)}$ G.D. Punkar, ${ }^{(27)}$ J. Quigley, ${ }^{(16)}$ B.N. Ratcliff, ${ }^{(27)}$ T.W. Reeves, ${ }^{(30)}$ J. Reidy, ${ }^{(18)}$ P.E. Rensing, ${ }^{(27)}$ L.S. Rochester, ${ }^{(27)}$ J.E. Rothberg, ${ }^{(31)}$ P.C. Rowson, ${ }^{(11)}$ J.J. Russell, ${ }^{(27)}$ O.H. Saxton, ${ }^{(27)}$ S.F. Schaffner, ${ }^{(27)}$ T. Schalk, ${ }^{(7)}$ R.H. Schindler, ${ }^{(27)}$ U. Schneekloth, ${ }^{(16)}$ B.A. Schumm, ${ }^{(15)}$ A. Seiden, ${ }^{(7)}$ S. Sen, ${ }^{(33)}$ V.V. Serbo, ${ }^{(32)}$ M.H. Shaevitz, ${ }^{(11)}$ J.T. Shank, ${ }^{(3)}$ G. Shapiro, ${ }^{(15)}$ S.L. Shapiro, ${ }^{(27)}$ D.J. Sherden, ${ }^{(27)}$ K.D. Shmakov, ${ }^{(28)}$ C. Simopoulos, ${ }^{(27)}$ N.B. Sinev, ${ }^{(20)}$ S.R. Smith, ${ }^{(27)}$ J.A. Snyder, ${ }^{(33)}$

P. Stamer, ${ }^{(25)}$ H. Steiner, ${ }^{(15)}$ R. Steiner, ${ }^{(1)}$ M.G. Strauss, ${ }^{(17)}$ D. Su, ${ }^{(27)}$

F. Suekane, ${ }^{(29)}$ A. Sugiyama, ${ }^{(19)}$ S. Suzuki, ${ }^{(19)}$ M. Swartz, ${ }^{(27)}$ A. Szumilo, ${ }^{(31)}$ T. Takahashi, ${ }^{(27)}$ F.E. Taylor, ${ }^{(16)}$ E. Torrence, ${ }^{(16)}$ J.D. Turk, ${ }^{(33)}$ T. Usher, ${ }^{(27)}$

J. Va'vra, ${ }^{(27)}$ C. Vannini, ${ }^{(23)}$ E. Vella, ${ }^{(27)}$ J.P. Venuti, ${ }^{(30)}$ R. Verdier,${ }^{(16)}$ P.G. Verdini, ${ }^{(23)}$ S.R. Wagner, ${ }^{(27)}$ A.P. Waite, ${ }^{(27)}$ S.J. Watts, ${ }^{(4)}$ A.W. Weidemann, ${ }^{(28)}$ E.R. Weiss, ${ }^{(31)}$ J.S. Whitaker, ${ }^{(3)}$ S.L. White, ${ }^{(28)}$ F.J. Wickens, ${ }^{(24)}$ D.A. Williams, ${ }^{(7)}$ D.C. Williams, ${ }^{(16)}$ S.H. Williams, ${ }^{(27)}$ S. Willocq, ${ }^{(33)}$ R.J. Wilson, ${ }^{(9)}$ W.J. Wisniewski, ${ }^{(5)}$ M. Woods, ${ }^{(27)}$ G.B. Word, ${ }^{(25)}$ J. Wyss, ${ }^{(21)}$ R.K. Yamamoto, ${ }^{(16)}$ J.M. Yamartino, ${ }^{(16)}$ X. Yang, ${ }^{(20)}$ S.J. Yellin, ${ }^{(6)}$ C.C. Young, ${ }^{(27)}$ H. Yuta, ${ }^{(29)}$ G. Zapalac, ${ }^{(32)}$ R.W. Zdarko, ${ }^{(27)}$ C. Zeitlin, ${ }^{(20)}$ Z. Zhang, ${ }^{(16)}$ and J. Zhou, ${ }^{(20)}$

(1) Adelphi University, Garden City, New York 11530

(2) INFN Sezione di Bologna, I-40126 Bologna, Italy

(3) Boston University, Boston, Massachusetts 02215

(4) Brunel University, Uxbridge, Middlesex UB8 3PH, United Kingdom . 
${ }^{(5)}$ California Institute of Technology, Pasadena, California 91125

(6) University of California at Santa Barbara, Santa Barbara, California 93106

(7) University of California at Santa Cruz, Santa Cruz, California 95064

${ }^{(8)}$ University of Cincinnati, Cincinnati, Ohio 45221

(9) Colorado State University, Fort Collins, Colorado 80523

${ }^{(10)}$ University of Colorado, Boulder, Colorado 80309

(11) Columbia University, New York, New York 10027

${ }^{(12)}$ INFN Sezione di Ferrara and Università di Ferrara, I-44100 Ferrara, Italy

(13) INFN Lab. Nazionali di Frascati, I-00044 Frascati, Italy

${ }^{(14)}$ University of Illinois, Urbana, Illinois 61801

${ }^{(15)}$ Lawrence Berkeley Laboratory, University of California, Berkeley, California 94720

${ }^{(16)}$ Massachusetts Institute of Technology, Cambridge, Massachusetts 02139

(17) University of Massachusetts, Amherst, Massachusetts 01003

(18) University of Mississippi, University, Mississippi 38677

(19) Nagoya University, Chikusa-ku, Nagoya 464 Japan

${ }^{(20)}$ University of Oregon, Eugene, Oregon 97403

${ }^{(21)}$ INFN Sezione di Padova and Università di Padova, I-35100 Padova, Italy

${ }^{(22)}$ INFN Sezione di Perugia and Università di Perugia, I-06100 Perugia, Italy

(23) INFN Sezione di Pisa and Università di Pisa, I-56100 Pisa, Italy

${ }^{(25)}$ Rutgers University, Piscataway, New Jersey 08855

${ }^{(24)}$ Rutherford Appleton Laboratory, Chilton, Didcot, Oxon OX11 0QX United Kingdom

${ }^{(26)}$ Sogang University, Seoul, Korea

(27) Stanford Linear Accelerator Center, Stanford University, Stanford, California 94309

(28) University of Tennessee, Knoxville, Tennessee 37996

${ }^{(29)}$ Tohoku University, Sendai 980 Japan

(30) Vanderbilt University, Nashville, Tennessee 37235

(31) University of Washington, Seattle, Washington 98195

(32) University of Wisconsin, Madison, Wisconsin 53706 
(33) Yale University, New Haven, Connecticut 06511

$\dagger$ Deceased

(a) Also at the Università di Genova

(b) Also at the Università di Perugia 


\section{Bibliography}

[1] G. Park, Implication of the Recent Top Quark Discovery on Two Higgs Doublet Model, HEP-PH-9504369, (1995).

[2] S. Abachi et. al., Observation of the Top Quark, HEP-EX-9503003, (1995).

[3] J. Liu and D. Ng, The effect of new physics on $R_{b}$, TRI-PP-94-71, (1994).

[4] M. Boulware and D. Finnel, Radiative Corrections to $B R(Z \rightarrow b \bar{b})$ in the Minimal Supersymmetric Standard Model, Phys. Rev. D44, 2054 (1991);

R. S. Chivukula, E. H. Simmons, and J. Terning, A Heavy Top Quark and the Z bb Vertex in Non-Commuting Extended Technicolor, Phys. Lett. B331, 383 (1994);

A. Akhundov, D. Bardin, and T. Riemann, Electroweak One Loop Corrections to the Decay of the Neutral Vector Boson, Nucl. Phys. B276, 1 (1986);

W. Beenakker, and W. Hollik, The Width of the Z Boson, Z. Phys. C40, 141 (1988);

B. W. Lynn, and R. G. Stuart, Electroweak Radiative Corrections to the b Quark Production, Phys. Lett. B252, 676 (1990).

[5] W. Hollik, The $Z$ Boson, the Top Quark, and Non-Minimal Higgs Bosons, Mod. Phys. Lett. A 5, 1909 (1990);

A. Djouadi et. al., $b \bar{b}$ Production on the $Z$ Resonance: A Challenge to the Standard Model, Nucl. Phys. B349, 48 (1991). 
[6] DELPHI Collaboration: P. Abreu et. al., Measurement of the $Z^{0}$ Branching Fraction to $b$ Quark Pairs Using the Boosted Sphericity Product, Phys. Lett. B281, 383 (1992).

[7] ALEPH Collaboration, D. Buskulic el. al., Heavy Flavor Production and Decay with Prompt Leptons in the Aleph Detector, Z. Phys C62, 179 (1994);

DELPHI Collaboration: P. Abreu et. al., Measurement of the Partial Width of the $Z^{0}$ into $b \bar{b}$ Final States Using their Semileptonic Decays, Z. Phys. C56, 47 (1992);

L3 Collaboration: B. Adeva et. al., Measurement of the Inclusive Production of Neutral Pions and Charged Particles on the $Z^{0}$ Resonance, Phys. Lett. B259, 199 (1991);

OPAL Collaboration: P. D. Acton et. al., Measurement of $\Gamma\left(Z^{0} \rightarrow b \bar{b}\right) / \Gamma\left(Z^{0} \rightarrow\right.$ hadrons) Using Leptons, Z. Phys. C58, 523 (1993).

[8] ALEPH Collaboration: D. Buskulic et. al, A Precise Measurement of $\Gamma\left(Z^{0} \rightarrow\right.$ $b \bar{b}) / \Gamma\left(Z^{0} \rightarrow\right.$ hadrons $)$, Phys.Lett. B313, 535 (1993);

OPAL Collaboration: P. D. Acton et. al, A Measurement of $\Gamma\left(Z^{0} \rightarrow b \bar{b}\right) / \Gamma\left(Z^{0} \rightarrow\right.$ hadrons) Using an Impact Parameter Technique, Z. Phys. C60, 579 (1993); OPAL Collaboration: D. Akers et. al, Measurement of $\Gamma\left(Z^{0} \rightarrow b \bar{b}\right) / \Gamma\left(Z^{0} \rightarrow\right.$ hadrons) Using a Double Tagging Method, CERN-PPE/94-106 (1994);

DELPHI Collaboration: P. Abreu et. al, Measurement of the $\Gamma\left(Z^{0} \rightarrow\right.$ $b \bar{b}) / \Gamma\left(Z^{0} \rightarrow\right.$ hadrons $)$ Branching Ratio of the $Z$ by Double Hemisphere Tagging, CERN-PPE/94-131 (1994).

[9] B. Schumm et. al., MLLA and the Average Charged Multiplicity of Events Containing Heavy Quarks in $e^{+} e^{-}$Annihilations, Phys. Rev. Lett. 69, 3025 (1992).

[10] F. Halzen and D. Martin, Quarks and Leptons: An Introductory Course in Modern Particle Physics, John Wiley and Sons, New York (1984).

[11] T. Junk, Measurement of $A_{b}$ from the Left-Right Forward-Backward Asymmetry of $b$-Quark Production in $Z^{0}$ Decays using a Momentum Weighted Track Charge Technique, Phys. Rev. Lett. 74, 2890 (1995). 
[12] N. Cooper and G. West, Particle Physics: A Los Alamos Primer, Cambridge University Press, Cambridge (1988).

[13] G. Punkar et. al., Measurement of the Average Lifetime of $B$ Hadrons at SLD, Presented at DPF 94, SLAC-PUB-6595 (1994).

[14] The Particle Data Group, Phys. Rev. D50, Part I (1994).

[15] J. Bernabéu, A. Pich, A. Santamaria, Top Quark Mass from Radiative Correction to the $Z^{0} \rightarrow b \bar{b}$ Decay, Phys. Lett. B363, 326 (1991).

[16] F. Boudjema, A. Djouadi, and C. Verzegnassi, A General Sum Rule for the Top Mass from $b$ Physics on $Z$ Resonance, Phys. Lett. B238, 423 (1990);

[17] M. Peskin, Theory of Precision Electroweak Measurements, Lecture presented at the Seventeenth SLAC Summer Institute, Physics at the $100 \mathrm{GeV}$ Mass Scale.

[18] Y. Okada, SUSY and CP Violation KEK-TH-428, (1995).

[19] M. Seymour, Heavy Quark Pair Multiplicity in $e^{+} e^{-}$Events, Nuclear Physics B436, 163 (1995).

[20] R. Koontz et. al., SLC-PUB-5890. Presented at Linac '92 Conference, Ottawa, Canada, Aug. 1992.

[21] M. Woods, POLARIZATION AT SLC, Presented at the Eleventh International Symposium on High Energy Spin Physics, SLAC-PUB-6694, (1995).

[22] M. Breidenbach et. al., SLC-PUB-6313. Presented at the 2nd International Workshop on Physics and Experiments with Linear Colliders, Waikala, Hawaii, Apr. 1993,; The SLD Collaboration, Proposal for an Extension of the SLD Study of Polarized $e^{+} e^{-}$Collisions at the SLC, Oct. 1993.

[23] K. Abe et. al., Measurements of $R_{b}$ using Impact Parameters and Displaced Vertices, SLAC-PUB-95-6569, (1995). 
[24] A. Benvenuti et. al., The Iron Calorimeter and Muon Identifier for SLD, Nucl. Instr. \& Meth. A276, 94 (1989).

[25] M. Hildreth et. al., SLAC-PUB-6656 Submitted to IEEE Trans. on Nucl. Sci. (1994).

[26] G. Agnew et. al., SLAC-PUB-5906 Proceedings of the 26th International Conference on High Energy Physics, Dallas, Texas, USA, Aug 1992.

[27] C. Damerell, Use of Charge-Coupled Devices in the Localisation of Particle Trajectories, Batavia 1981, Proceedings, Silicon Detectors For High Energy Physics*, 33 (1981);

C. Damerell, F. Farley, A. Gillman, and F. Wickens, Charge-Coupled Devices for Particle Detection with High Spatial Resolution Nucl. Instr. \& Meth. 185, 33 (1981).

[28] E. Vella et. al., SLD Liquid Argon Calorimeter, Presented at the III International Conference on Calorimetry in High Energy Physics, SLAC-PUB-5953 (1992); D. Axen et. al., Nucl. Instr. \& Meth. A328, 472 (1993).

[29] The SLD Collaboration, A Status Report on the SLD Data Acquisition System, IEEE Trans. on Nucl. Sci. 36, 23 (1989).

[30] S. Wagner, SLD Collaboration Meeting Proceedings, May 1993.

[31] GEANT 3.15 Program, CERN Application Software Group, CERN Program Library (1993), GEANT 3.21 Program, CERN Application Software Group, CERN Program Library (1994).

[32] T. Sjöstrand, and M. Bengtsson, Comp. Phys. Comm. 43, 367 (1987);

T. Sjöstrand, Comp. Phys. Comm. 39, 347 (1986).

[33] M. Artuso (CLEO Collab.), HEPSY-7-93, talk presented at Workshop on $b$ Physics at Hadron Accelerators, July 1993. 
[34] T. Junk, SLD Collaboration Meeting Proceedings, Chateau La Cresta, CA, Feb. 1995.

[35] R. Wang, Ph.D thesis, University of Minnesota (1994).

[36] M. Thulasidas, Ph.D thesis, Syracuse University (1993).

[37] N. Isgur, D. Scora, B. Grinstein, and M. B. Wise, Semileptonic $B$ and D Decays in the Quark Model, Phys. Rev. D39, 799 (1989);

Code provided by P. Kim and CLEO Collaboration.

[38] The Particle Data Group, Phys. Rev. D45, Part II (1992).

[39] LEP Electroweak Heavy Flavor Group's Recommendations (1994).

[40] MARK-III Collaboration: D. Coffman et. al., Measurement of the Inclusive Decay Properties of Charmed Mesons, Phys. Lett. B263, 135 (1991).

[41] CLEO Collaboration: F. Butler et. al, Measurement of the $D^{*}(2010)$ Branching Fractions, Phys. Rev. Lett. 69, 2041 (1992).

[42] M. Wirbel, B. Stech, and M. Bauer, Exclusive Semileptonic Decays of Heavy Mesons, Z. Phys. C29, 637 (1985).

[43] TASSO Collaboration: W. Braunschweig et. al., Jet Fragmentation and QCD Models in $e^{+} e^{-}$Annihilation at C.M. Energies Between $12 \mathrm{GeV}$ and $41.5 \mathrm{GeV}$, Z. Phys. C41, 359 (1988);

P. N. Burrows, Predictions of QCD Fragmentation Models in $e^{+} e^{-}$Annihilation up to $\sqrt{s}>200 \mathrm{GeV}$, Z. Phys. C41, 375 (1988).

[44] OPAL Collaboration: M. Z. Akrawy et. al., A Measurement of Global Event Shape Distributions in the Hadronic Decays of the $Z^{0}$, Z. Phys. C47, 505 (1990).

[45] C. Peterson et. al., Scaling violations in inclusive $e^{+} e^{-}$annihilations spectra, Phys. Rev. D27, 105 (1983). 
[46] ALEPH Collaboration, D. Buskulic el. al., Heavy Flavor Production and Decay with Prompt Leptons in the ALEPH Detector, Z. Phys. C62, 179 (1994);

L3 Collaboration: B. Adeva et. al., Measurements $Z^{0} \rightarrow b \bar{b}$ Decays and the Semileptonic Branching Ratio $\operatorname{Br}(B \rightarrow$ LeptonX $)$, Phys.Lett. B261, 177 (1991);

ALEPH Collaboration: D. Buskulic et.al., Production of Charmed Mesons in $Z$ Decays, Z. Phys. C62, 1 (1994).

[47] ALEPH Collaboration: D. Decamp et. al., Phys. Lett. B273, 181 (1991);

DELPHI Collaboration: P. Abreu et. al., Z. Phys. C50, 185 (1991);

L3 Collaboration: B. Adeva et. al., Measurement of the Inclusive Production of Neutral Pions and Charged Particles on the $Z^{0}$ Resonance, Phys. Lett. B259, 199 (1991);

OPAL Collaboration: P. D. Acton et. al., A Study of Charged Particle Multiplicities in Hadronic Decays of the $Z^{0}, Z$. Phys. C53, 539 (1992).

[48] MARK-II Collaboration: R. Jacobsen et. al., Measurement of the $b \bar{b}$ Fraction in Hadronic $Z^{0}$ Decays with Precision Vertex Detectors, Phys. Rev. Lett. 67, 3347 (1991).

[49] JADE Collaboration: S. Bethke et. al., Experiemental Investigation of the Energy Dependence of the Strong Couplijng Strength, Phys. Lett. B213, 235 (1988).

[50] ALEPH, Numbers, (An ALEPH WWW page (http://alephwww.cern.ch /ALEPHGENERAL/reports/alephnum/alephnum.html)) (1995).

[51] V. Luth, Vertex Detectors, Lecture presented at the SLAC Summer Institute (1992).

[52] M. G. Bowler, $e^{+} e^{-}$Production of Heavy Quarks in the String Model, Z. Phys. C11, 169 (1981); 